\title{
Phospha-Claisen Type Reactions at Frustrated Lewis Pair Frameworks
}

Guo-Qiang Chen, Gerald Kehr, Christian Mück-Lichtenfeld, Constantin G. Daniliuc, Gerhard Erker*

Organisch-Chemisches Institut, Universität Münster, Corrensstrasse 40, 48149 Münster, Germany.

\section{Supporting Information}

General Information S2

Synthesis of compound 8a S3

Synthesis of compound $8 \mathrm{~b}$ S7

Synthesis of compound 8c S12

Control experiments (4a + 5a,b,c: NMR scale) S15

$\begin{array}{lr}\text { Synthesis of compound 10a } & \text { S21 }\end{array}$

$\begin{array}{lr}\text { Synthesis of compound 10b } & \text { S25 }\end{array}$

$\begin{array}{lr}\text { Synthesis of compound 11a } & \text { S29 }\end{array}$

Control experiments (4b + 9b: NMR scale) $\quad$ S34

$\begin{array}{ll}\text { Synthesis of compound } 12 & \mathbf{S 3 7}\end{array}$

$\begin{array}{ll}\text { Synthesis of compound } 16 & \text { S43 }\end{array}$

Control experiments (4c + 9b: NMR scale) $\quad$ S48

Control experiments: heating of compounds

8b, 10a, and 11a in $\mathrm{C}_{6} \mathrm{D}_{6}$ (NMR scale) $\quad \mathrm{S50}$

$\begin{array}{lr}\text { DFT Calculations } & \mathbf{S 5 7}\end{array}$ 
General Procedure. All syntheses involving air- and moisture sensitive compounds were carried out using standard Schlenk-type glassware (or in a glove box) under an atmosphere of argon. Solvents were dried and stored under an argon atmosphere. NMR spectra were recorded on an Agilent DD2-500 MHz $\left({ }^{1} \mathrm{H}: 500 \mathrm{MHz},{ }^{13} \mathrm{C}: 126 \mathrm{MHz},{ }^{19} \mathrm{~F}\right.$ : $\left.470 \mathrm{MHz},{ }^{11} \mathrm{~B}: 160 \mathrm{MHz},{ }^{31} \mathrm{P}: 202 \mathrm{MHz}\right)$ and on a Agilent DD2-600 MHz $\left({ }^{1} \mathrm{H}: 600\right.$ $\left.\mathrm{MHz},{ }^{13} \mathrm{C}: 151 \mathrm{MHz},{ }^{19} \mathrm{~F}: 564 \mathrm{MHz},{ }^{11} \mathrm{~B}: 192 \mathrm{MHz},{ }^{31} \mathrm{P}: 243 \mathrm{MHz}\right) .{ }^{1} \mathrm{H} \mathrm{NMR}$ and ${ }^{13} \mathrm{C}$ NMR: chemical shifts are given relative to TMS and referenced to the solvent signal. ${ }^{19} \mathrm{~F}$ NMR: chemical shifts are given relative to $\mathrm{CFCl}_{3}(\delta=0$, external reference $),{ }^{11} \mathrm{~B}$ NMR: chemical shifts are given relative to $\mathrm{BF}_{3} \cdot \mathrm{Et}_{2} \mathrm{O}\left(\delta=0\right.$, external reference), ${ }^{31} \mathrm{P}$ NMR: chemical shifts are given relative to $\mathrm{H}_{3} \mathrm{PO}_{4}\left(85 \%\right.$ in $\left.\mathrm{D}_{2} \mathrm{O}\right)(\delta=0$, external reference). NMR assignments were supported by additional 2D NMR experiments. Elemental analyses were performed on an Elementar Vario El III. Melting points and decomposition points were obtained with a DSC 2010 (TA Instruments).

X-Ray diffraction: For compounds 11a, 12 and 16 data sets were collected with a Nonius Kappa CCD diffractometer. Programs used: data collection, COLLECT (R. W. W. Hooft, Bruker AXS, 2008, Delft, The Netherlands); data reduction Denzo-SMN (Z. Otwinowski, W. Minor, Methods Enzymol. 1997, 276, 307-326); absorption correction, Denzo (Z. Otwinowski, D. Borek, W. Majewski, W. Minor, Acta Crystallogr. 2003, A59, 228-234); structure solution SHELXS-97 (G. M. Sheldrick, Acta Crystallogr. 1990, A46, 467-473); structure refinement SHELXL-97 (G. M. Sheldrick, Acta Crystallogr. 2008, A64, 112-122) and graphics, XP (BrukerAXS, 2000). For compound 8a data sets were collected with a Kappa CCD APEXII Bruker diffractometer. For compound $\mathbf{8 b}$ data sets were collected with a D8 Venture Dual Source 100 CMOS diffractometer. Programs used: data collection: APEX2 V2014.5-0 (Bruker AXS Inc., 2014); cell refinement: SAINT V8.34A (Bruker AXS Inc., 2013); data reduction: SAINT V8.34A (Bruker AXS Inc., 2013); absorption correction, SADABS V2014/2 (Bruker AXS Inc., 2014); structure solution SHELXT-2014 (Sheldrick, 2014); structure refinement SHELXL-2014 (Sheldrick, 2014) and graphics, XP (Bruker AXS Inc., 2014). $R$-values are given for observed reflections, and $w \mathrm{R}^{2}$ values are given for all reflections. Exceptions and special features: For compound $\mathbf{8 b}$ two badly disordered dichloromethane molecules and for compound 11a one and a half badly disordered dichloromethane molecules were found in the asymmetrical unit and could not be satisfactorily refined. The program SQUEEZE (A. L. Spek J. Appl. Cryst., 2003, 36, 713) was therefore used to remove mathematically the effect of the solvent. The quoted formula and derived parameters are not included the squeezed solvent molecules.

Materials. P/B FLPs 4a-c [Moquist, P.; Chen, G.-Q.; Mück-Lichtenfeld, C.; Bussmann, K.; Daniliuc, C. G.; Kehr, G.; Erker, G. Chem. Sci. 2015, 6, 816-825.] were prepared according to the literature. 


\section{Synthesis of compound 8a}

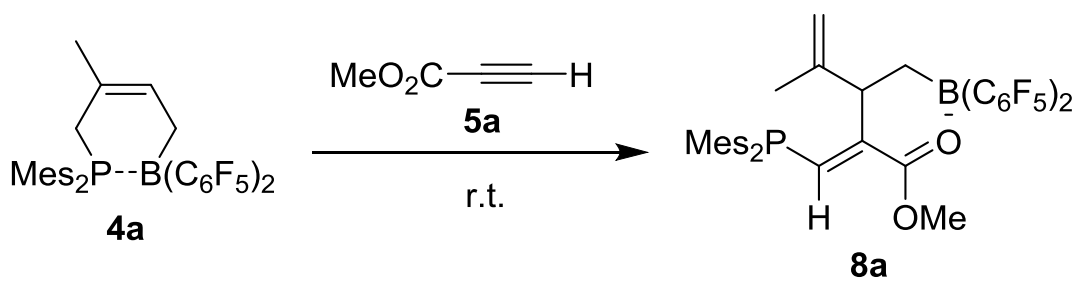

\section{Scheme S1}

A solution of compounds $4 \mathbf{a}(102.3 \mathrm{mg}, 0.15 \mathrm{mmol})$ and $\mathbf{5 a}(12.6 \mathrm{mg}, 0.15 \mathrm{mmol})$ in dichloromethane $(3 \mathrm{~mL})$ was stirred at room temperature for $5 \mathrm{~h}$. Then all volatiles were removed in vacuo and compound $\mathbf{8 a}(103.6 \mathrm{mg}, 90 \%)$ was obtained as a yellow solid. Crystals suitable for the X-ray crystal structure analysis were obtained by slow diffusion of pentane to a solution of compound 8a in dichloromethane at $-35{ }^{\circ} \mathrm{C}$. Decomp. 157 ${ }^{\circ} \mathrm{C}$. Anal. Calc. for $\mathrm{C}_{39} \mathrm{H}_{34} \mathrm{BF}_{10} \mathrm{O}_{2} \mathrm{P}: \mathrm{C}: 61.12 ; \mathrm{H}: 4.47$. Found: C: 60.36; H: 4.50 .

${ }^{1} \mathbf{H}$ NMR $\left(500 \mathrm{MHz}, 299 \mathrm{~K}, \mathrm{C}_{6} \mathrm{D}_{6}\right): \delta=8.23\left(\mathrm{dd},{ }^{2} J_{\mathrm{PH}}=7.1 \mathrm{~Hz},{ }^{4} J_{\mathrm{HH}}=1.4 \mathrm{~Hz}, 1 \mathrm{H}\right.$, $=\mathrm{CH}), 6.60\left(\mathrm{dm},{ }^{4} \mathrm{~J}_{\mathrm{PH}}=3.5 \mathrm{~Hz}, 4 \mathrm{H}, m-\mathrm{Mes}\right), 4.40\left(\mathrm{~m}, 1 \mathrm{H},=\mathrm{CH}_{2}{ }^{\mathrm{E}}\right), 4.35\left(\mathrm{~s}, 1 \mathrm{H},=\mathrm{CH}_{2}^{\mathrm{Z}}\right)$, $3.33\left(\mathrm{~d}, J=0.8 \mathrm{~Hz}, 3 \mathrm{H}, \mathrm{OCH}_{3}\right), 3.03(\mathrm{~m}, 1 \mathrm{H}, \mathrm{CH}), 2.15\left(\mathrm{~s}, 12 \mathrm{H}, o-\mathrm{CH}_{3}{ }^{\mathrm{Mes}}\right), 2.01(\mathrm{~s}, 6 \mathrm{H}$, $\left.p-\mathrm{CH}_{3}{ }^{\mathrm{Mes}}\right), 1.79\left(\mathrm{dd},{ }^{2} J_{\mathrm{HH}}=15.1 \mathrm{~Hz},{ }^{3} J_{\mathrm{HH}}=6.3 \mathrm{~Hz}, 1 \mathrm{H}, \mathrm{CH}_{2}\right), 1.59\left(\mathrm{dd},{ }^{2} J_{\mathrm{HH}}=15.1 \mathrm{~Hz}\right.$, $\left.{ }^{3} J_{\mathrm{HH}}=7.6 \mathrm{~Hz}, 1 \mathrm{H}, \mathrm{CH}_{2}\right), 1.24\left(\mathrm{~s}, 3 \mathrm{H}, \mathrm{CH}_{3}\right)$.

${ }^{13} \mathbf{C}\left\{{ }^{1} \mathbf{H}\right\}$ NMR $\left(126 \mathrm{MHz}, 299 \mathrm{~K}, \mathrm{C}_{6} \mathrm{D}_{6}\right): \delta=174.9\left(\mathrm{~d},{ }^{3} J_{\mathrm{PC}}=9.5 \mathrm{~Hz}, \mathrm{C}=\mathrm{OB}\right), 158.9(\mathrm{~d}$, $\left.{ }^{1} J_{\mathrm{PC}}=20.5 \mathrm{~Hz},=\mathrm{CH}\right), 146.1\left(\mathrm{~d},{ }^{4} J_{\mathrm{PC}}=3.0 \mathrm{~Hz}, \mathrm{C}={ }^{\mathrm{CH} 2}\right), 143.1\left(\mathrm{~d},{ }^{2} J_{\mathrm{PC}}=15.3 \mathrm{~Hz}, o-\mathrm{Mes}\right)$, $140.0\left(\mathrm{~d},{ }^{4} J_{\mathrm{PC}}=0.8 \mathrm{~Hz}, p\right.$-Mes $), 130.3\left(\mathrm{~d},{ }^{3} J_{\mathrm{PC}}=4.8 \mathrm{~Hz}, m-\mathrm{Mes}\right), 128.9\left(\mathrm{~d},{ }^{2} J_{\mathrm{PC}}=19.1\right.$ $\left.\mathrm{Hz}, \mathrm{C}={ }^{\mathrm{CH}}\right), 128.0(i-\mathrm{Mes}), 110.6\left(\mathrm{~d},{ }^{5} J_{\mathrm{PC}}=1.4 \mathrm{~Hz},=\mathrm{CH}_{2}\right), 56.8\left(\mathrm{OCH}_{3}\right), 42.3\left(\mathrm{~d},{ }^{3} J_{\mathrm{PC}}=\right.$ $3.3 \mathrm{~Hz}, \mathrm{CH}), 23.5\left(\mathrm{~d},{ }^{3} J_{\mathrm{PC}}=14.2 \mathrm{~Hz}, o-\mathrm{CH}_{3}{ }^{\mathrm{Mes}}\right), 22.2\left(\mathrm{br}, \mathrm{CH}_{2}\right), 20.8\left(p-\mathrm{CH}_{3}{ }^{\mathrm{Mes}}\right), 20.4$ $\left(\mathrm{d},{ }^{5} \mathrm{JPC}_{\mathrm{PC}}=1.0 \mathrm{~Hz}, \mathrm{CH}_{3}\right),\left[\mathrm{C}_{6} \mathrm{~F}_{5}\right.$ not listed $]$.

${ }^{11} \mathbf{B}\left\{{ }^{1} \mathbf{H}\right\}$ NMR $\left(160 \mathrm{MHz}, 299 \mathrm{~K}, \mathrm{C}_{6} \mathrm{D}_{6}\right): \delta=3.4\left(v_{1 / 2} \sim 700 \mathrm{~Hz}\right)$.

${ }^{31} \mathbf{P}\left\{{ }^{1} \mathbf{H}\right\}$ NMR $\left(202 \mathrm{MHz}, 299 \mathrm{~K}, \mathrm{C}_{6} \mathrm{D}_{6}\right): \delta=-28.9\left(v_{1 / 2} \sim 2 \mathrm{~Hz}\right)$.

${ }^{19}$ F NMR $\left(470 \mathrm{MHz}, 299 \mathrm{~K}, \mathrm{C}_{6} \mathrm{D}_{6}\right): \delta=-134.5(\mathrm{~m}, 2 \mathrm{~F}, o),-158.5\left(\mathrm{t},{ }^{3} J_{\mathrm{FF}}=20.6 \mathrm{~Hz}, 1 \mathrm{~F}\right.$, $p),-163.9(\mathrm{~m}, 2 \mathrm{~F}, m)\left(\mathrm{C}_{6} \mathrm{~F}_{5}\right)\left[\Delta \delta^{19} \mathrm{~F}_{\mathrm{mp}}=5.4\right],-135.3(\mathrm{~m}, 2 \mathrm{~F}, o),-159.3\left(\mathrm{t},{ }^{3} J_{\mathrm{FF}}=20.7 \mathrm{~Hz}\right.$, $1 \mathrm{~F}, p),-164.7(\mathrm{~m}, 2 \mathrm{~F}, m)\left(\mathrm{C}_{6} \mathrm{~F}_{5}\right)\left[\Delta \delta^{19} \mathrm{~F}_{\mathrm{mp}}=5.4\right]$.

${ }^{1} \mathbf{H},{ }^{1} \mathbf{H}$-GCOSY $\left(500 \mathrm{MHz} / 500 \mathrm{MHz}, 299 \mathrm{~K}, \mathrm{C}_{6} \mathrm{D}_{6}\right)\left[\right.$ selected traces]: $\delta{ }^{1} \mathrm{H} / \delta^{1} \mathrm{H}=$ 4.40, $4.35 / 1.24\left(=\mathrm{CH}_{2}{ }^{\mathrm{E}},=\mathrm{CH}_{2}{ }^{\mathrm{Z}} / \mathrm{CH}_{3}\right), 3.03 / 1.79,1.59\left(\mathrm{CH} / \mathrm{CH}_{2}, \mathrm{CH}_{2}\right)$.

${ }^{1} \mathbf{H},{ }^{13} \mathbf{C}-G H S Q C\left(500 \mathrm{MHz} / 126 \mathrm{MHz}, 299 \mathrm{~K}, \mathrm{C}_{6} \mathrm{D}_{6}\right): \delta{ }^{1} \mathrm{H} / \delta{ }^{13} \mathrm{C}=8.23 / 158.9$ $(=\mathrm{CH}), 6.60 / 130.3$ (m-Mes), 4.40, $4.35 / 110.6\left(=\mathrm{CH}_{2}\right), 3.33 / 56.8\left(\mathrm{OCH}_{3}\right), 3.03 / 42.3$ $(\mathrm{CH}), 2.15 / 23.5\left(o-\mathrm{CH}_{3}{ }^{\mathrm{Mes}}\right), 2.01 / 20.8\left(p-\mathrm{CH}_{3}{ }^{\mathrm{Mes}}\right), 1.79,1.59 / 22.2\left(\mathrm{CH}_{2}\right), 1.24 / 20.4$ $\left(\mathrm{CH}_{3}\right)$.

${ }^{1} \mathbf{H},{ }^{13} \mathbf{C}$-GHMBC (500 MHz / $\left.126 \mathrm{MHz}, 299 \mathrm{~K}, \mathrm{C}_{6} \mathrm{D}_{6}\right)$ [selected traces]: $\delta{ }^{1} \mathrm{H} / \delta{ }^{13} \mathrm{C}=$ 8.23, 6.60, $2.15 / 128.0$ ( $=\mathrm{CH}, m$-Mes, $o-\mathrm{CH}_{3}{ }^{\mathrm{Mes}} / i$-Mes), 8.23, 3.33, 3.03 / 174.9 ( $=\mathrm{CH}$, $\left.\mathrm{OCH}_{3}, \mathrm{CH} / \mathrm{C}=\mathrm{OB}\right), 8.23,3.03,1.79,1.59 / 128.9\left(=\mathrm{CH}, \mathrm{CH}, \mathrm{CH}_{2}, \mathrm{CH}_{2} / \mathrm{C}={ }^{\mathrm{CH}}\right), 4.35$, 
3.03, 1.79, 1.59, $1.24 / 146.1\left(=\mathrm{CH}_{2}{ }^{\mathrm{Z}}, \mathrm{CH}, \mathrm{CH}_{2}, \mathrm{CH}_{2}, \mathrm{CH}_{3} / \mathrm{C}={ }^{\mathrm{CH}} 2\right), 2.15 / 143.1(o-$ $\mathrm{CH}_{3}{ }^{\mathrm{Mes}} / o$-Mes), $2.01 / 140.0$ ( $p$ - $\mathrm{CH}_{3}{ }^{\mathrm{Mes}} / p$-Mes).

${ }^{19} \mathbf{F},{ }^{19} \mathbf{F}-G C O S Y\left(470 \mathrm{MHz} / 470 \mathrm{MHz}, 299 \mathrm{~K}, \mathrm{C}_{6} \mathrm{D}_{6}\right): \delta{ }^{19} \mathrm{~F} / \delta{ }^{19} \mathrm{~F}=-163.9 /-134.5$, $158.5\left(m-\mathrm{C}_{6} \mathrm{~F}_{5}^{\mathrm{a}} / o-\mathrm{C}_{6} \mathrm{~F}_{5}{ }^{\mathrm{a}}, p-\mathrm{C}_{6} \mathrm{~F}_{5}^{\mathrm{a}}\right),-164.7 /-135.3,-159.3\left(m-\mathrm{C}_{6} \mathrm{~F}_{5} \mathrm{~b} / o-\mathrm{C}_{6} \mathrm{~F}_{5}^{\mathrm{b}}, p-\mathrm{C}_{6} \mathrm{~F}_{5}{ }^{\mathrm{b}}\right)$.

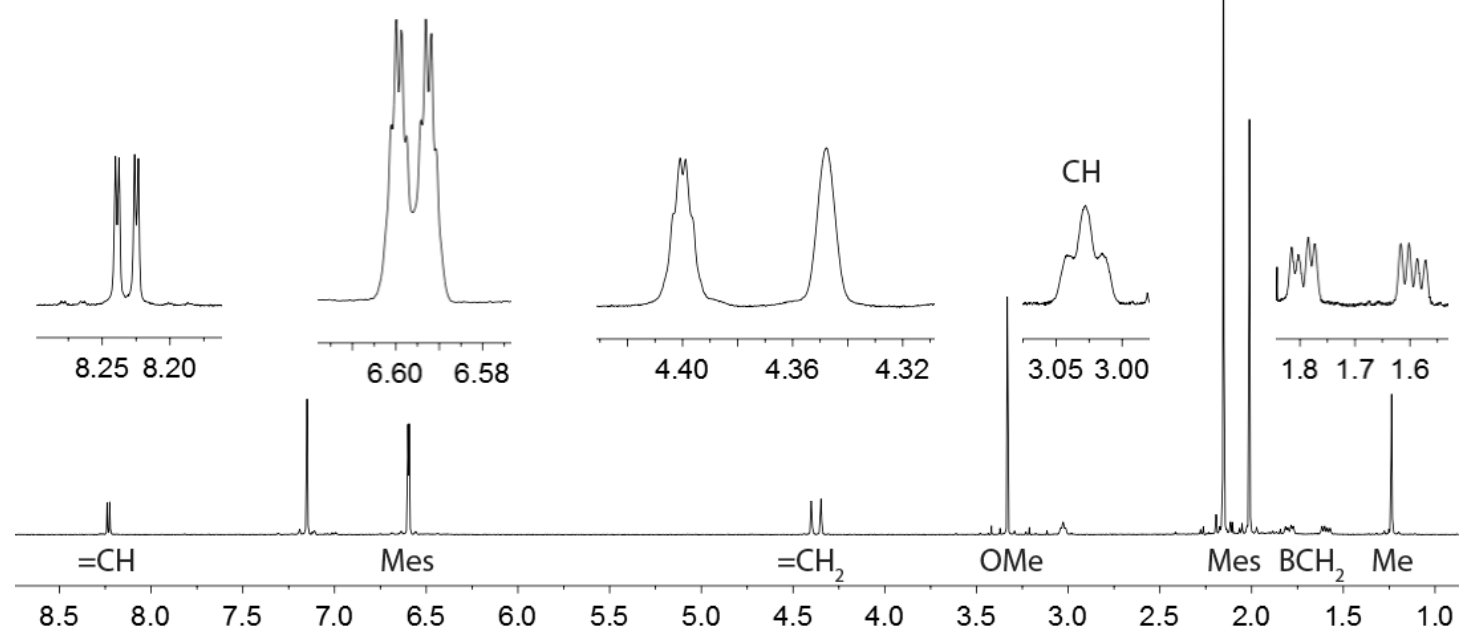

Figure S1. ${ }^{1} \mathbf{H}$ NMR $\left(500 \mathrm{MHz}, 299 \mathrm{~K}, \mathrm{C}_{6} \mathrm{D}_{6}\right)$ of compound 8a

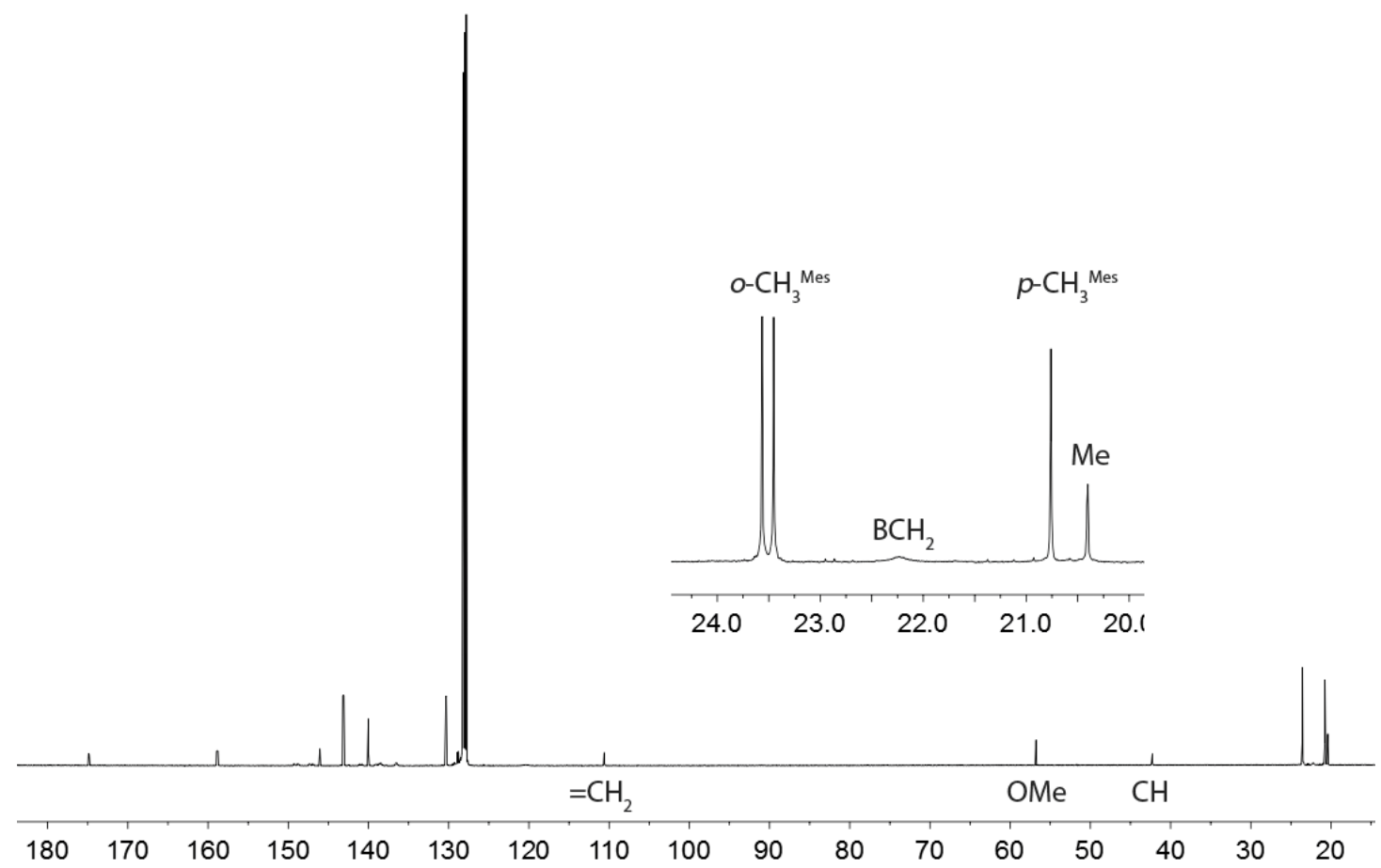

Figure S2. ${ }^{13} \mathbf{C}\left\{{ }^{1} \mathbf{H}\right\}$ NMR $\left(126 \mathrm{MHz}, 299 \mathrm{~K}, \mathrm{C}_{6} \mathrm{D}_{6}\right)$ of compound 8a 


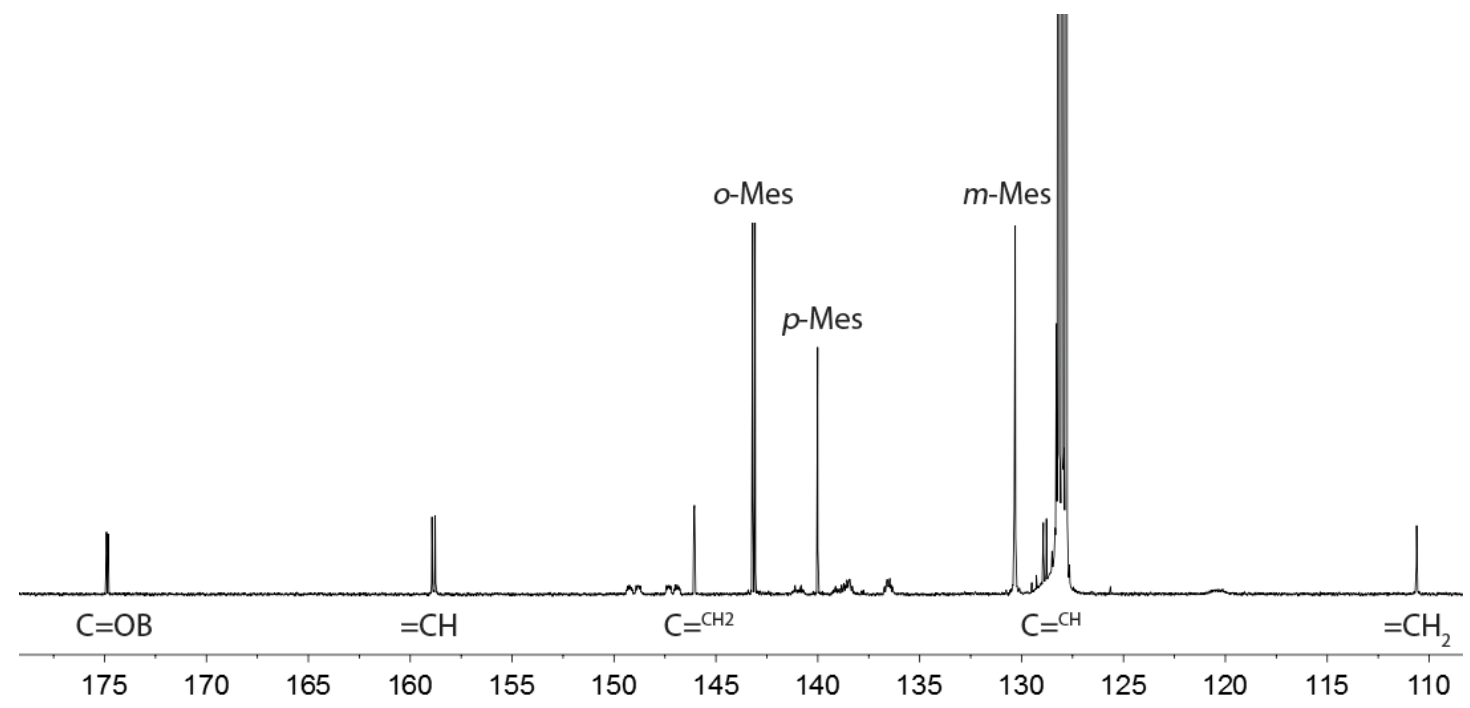

Figure S3. ${ }^{13} \mathbf{C}\left\{{ }^{1} \mathbf{H}\right\}$ NMR $\left(126 \mathrm{MHz}, 299 \mathrm{~K}, \mathrm{C}_{6} \mathrm{D}_{6}\right)$ of compound 8a

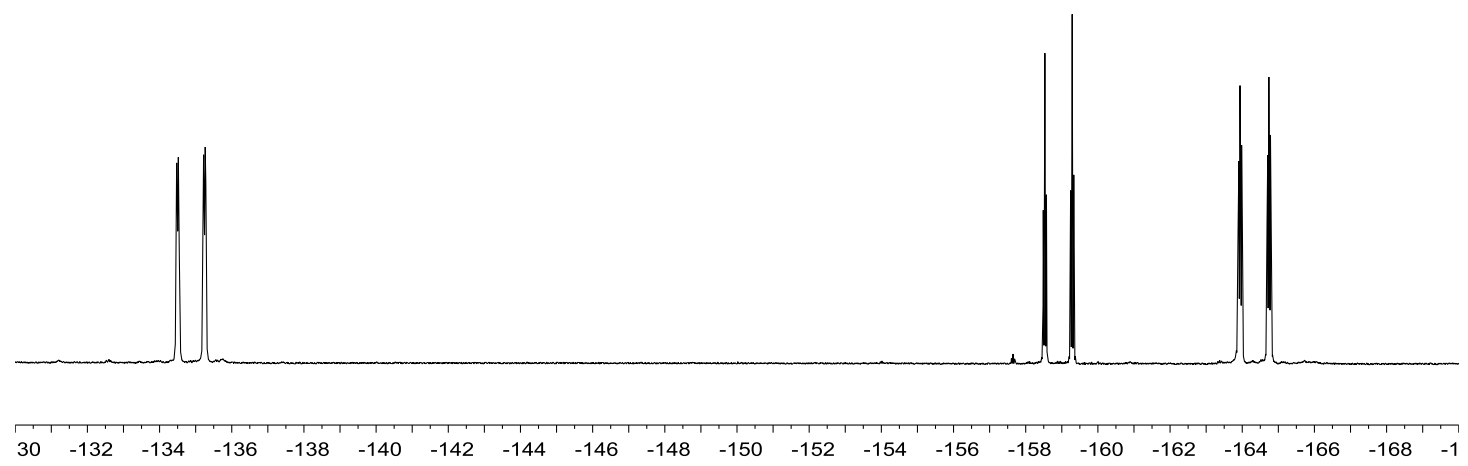

Figure S4. ${ }^{19}$ F NMR $\left(470 \mathrm{MHz}, 299 \mathrm{~K}, \mathrm{C}_{6} \mathrm{D}_{6}\right)$ of compound $\mathbf{8 a}$

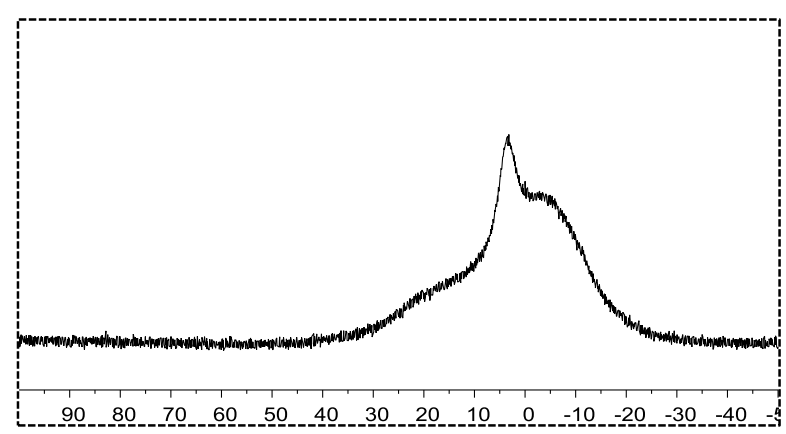

$\begin{array}{lllllllllllllllll}220 & 200 & 180 & 160 & 140 & 120 & 100 & 80 & 60 & 40 & 20 & 0 & -20 & -40 & -60 & -80 & -10\end{array}$

Figure S5. ${ }^{11} \mathbf{B}\left\{{ }^{1} \mathbf{H}\right\}$ NMR $\left(160 \mathrm{MHz}, 299 \mathrm{~K}, \mathrm{C}_{6} \mathrm{D}_{6}\right)$ and ${ }^{31} \mathbf{P}\left\{{ }^{1} \mathbf{H}\right\}$ NMR $(202 \mathrm{MHz}, 299$ $\mathrm{K}, \mathrm{C}_{6} \mathrm{D}_{6}$ ) of compound $\mathbf{8 a}$ 
X-ray crystal structure analysis of compound 8a: A yellow plate-like specimen of $\mathrm{C}_{39} \mathrm{H}_{34} \mathrm{BF}_{10} \mathrm{O}_{2} \mathrm{P}$, approximate dimensions $0.020 \mathrm{~mm} \times 0.100 \mathrm{~mm} \times 0.120 \mathrm{~mm}$, was used for the X-ray crystallographic analysis. The X-ray intensity data were measured. A total of 1140 frames were collected. The total exposure time was 25.93 hours. The frames were integrated with the Bruker SAINT software package using a wide-frame algorithm. The integration of the data using a monoclinic unit cell yielded a total of 31721 reflections to a maximum $\theta$ angle of $65.08^{\circ}$ ( $0.85 \AA$ resolution), of which 6011 were independent (average redundancy 5.277, completeness $=99.4 \%, \mathrm{R}_{\text {int }}=9.03 \%, \mathrm{R}_{\text {sig }}=$ $6.89 \%)$ and $3973(66.10 \%)$ were greater than $2 \sigma\left(\mathrm{F}^{2}\right)$. The final cell constants of $\underline{\mathrm{a}}=$ 11.4134(15) $\AA, \underline{b}=20.663(3) \AA, \underline{c}=15.422(2) \AA, \beta=102.950(8)^{\circ}$, volume $=3544.5(8)$ $\AA^{3}$, are based upon the refinement of the XYZ-centroids of 4481 reflections above 20 $\sigma(\mathrm{I})$ with $7.273^{\circ}<2 \theta<132.9^{\circ}$. Data were corrected for absorption effects using the multi-scan method (SADABS). The ratio of minimum to maximum apparent transmission was 0.849. The calculated minimum and maximum transmission coefficients (based on crystal size) are 0.8430 and 0.9710 . The final anisotropic fullmatrix least-squares refinement on $\mathrm{F}^{2}$ with 486 variables converged at $\mathrm{R} 1=5.24 \%$, for the observed data and $w \mathrm{R} 2=13.91 \%$ for all data. The goodness-of-fit was 1.065 . The largest peak in the final difference electron density synthesis was $0.258 \mathrm{e}^{-} / \AA^{3}$ and the largest hole was $-0.341 \mathrm{e}^{-} / \AA^{3}$ with an RMS deviation of $0.065 \mathrm{e}^{-} / \AA^{3}$. On the basis of the final model, the calculated density was $1.436 \mathrm{~g} / \mathrm{cm}^{3}$ and $\mathrm{F}(000), 1576 \mathrm{e}^{-}$.

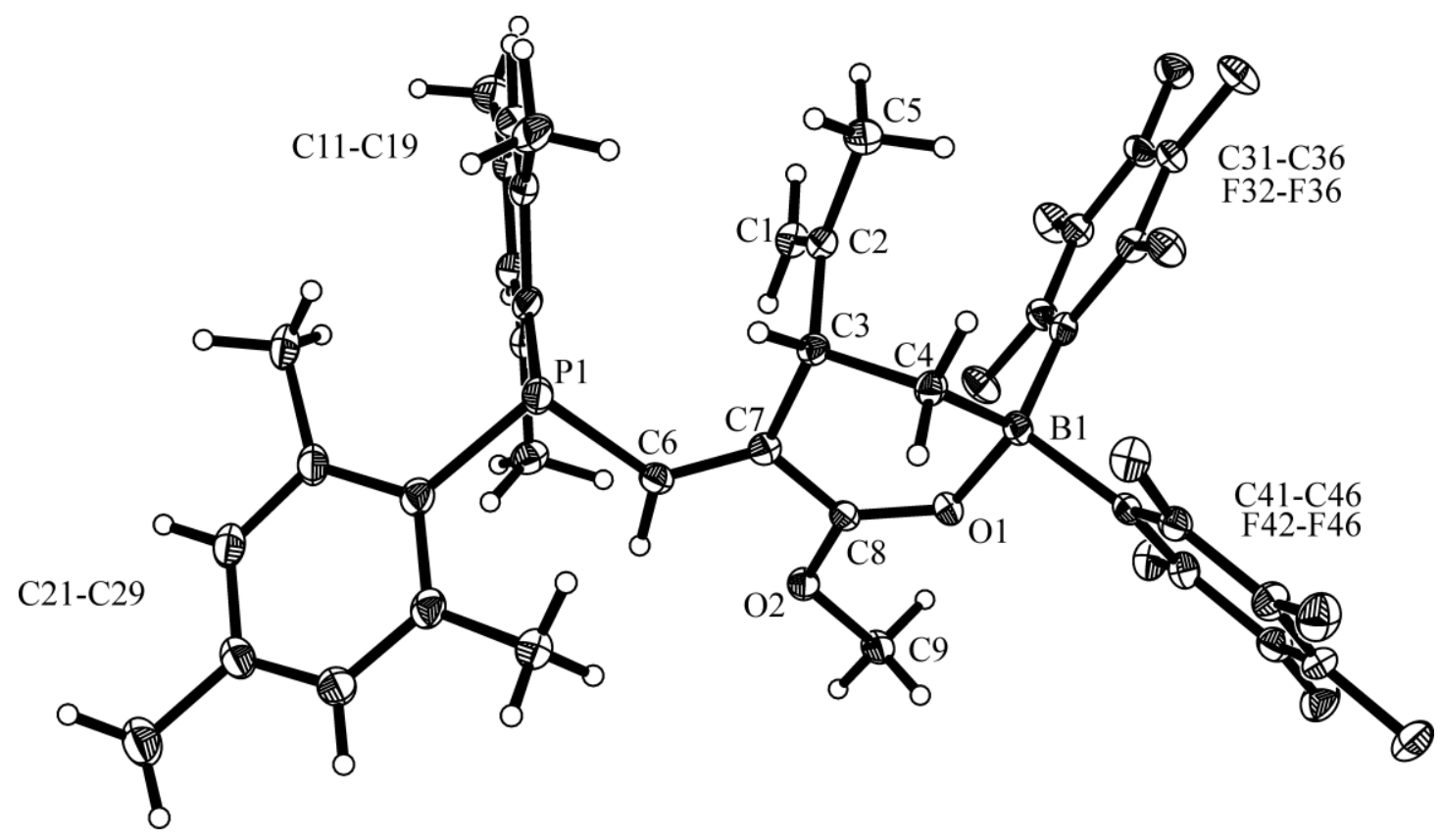

Figure S6. 


\section{Synthesis of compound $8 b$}

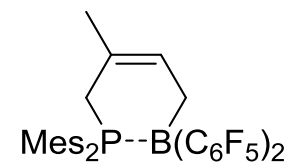

$4 a$

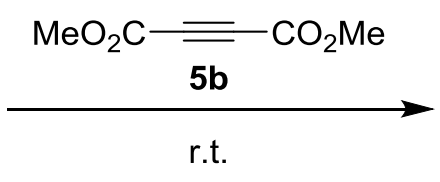

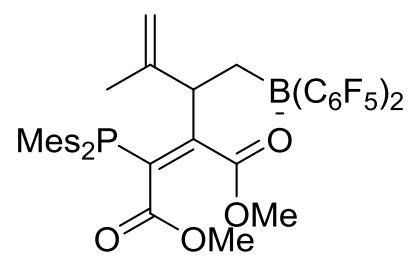

8b

\section{Scheme S2}

A solution of compounds $\mathbf{4 a}(102.3 \mathrm{mg}, 0.15 \mathrm{mmol})$ and $\mathbf{5 b}(21.3 \mathrm{mg}, 0.15 \mathrm{mmol})$ in pentane $(3 \mathrm{~mL})$ was stirred at room temperature for $1 \mathrm{~h}$. Then all volatiles were removed in vacuo and the residue was washed with cold pentane $(3 \times 1 \mathrm{~mL})$. After drying in vacuo compound $\mathbf{8 b}$ (102.6 mg, $83 \%$ ) was obtained as an orange solid. Crystals suitable for the X-ray crystal structure analysis were obtained by slow diffusion of pentane to a solution of compound $8 \mathbf{b}$ in dichloromethane at $-35^{\circ} \mathrm{C}$. Decomp. $179{ }^{\circ} \mathrm{C}$. Anal. Calc. for $\mathrm{C}_{41} \mathrm{H}_{36} \mathrm{BF}_{10} \mathrm{O}_{4} \mathrm{P}: \mathrm{C}: 59.73$; $\mathrm{H}: 4.40$. Found: C: 59.11; H: 4.22.

${ }^{1} \mathbf{H}$ NMR $\left(500 \mathrm{MHz}, 299 \mathrm{~K}, \mathrm{C}_{6} \mathrm{D}_{6}\right): \delta=6.61\left(\mathrm{dm},{ }^{4} J_{\mathrm{PH}}=3.6 \mathrm{~Hz}, 4 \mathrm{H}, m-\mathrm{Mes}\right), 4.52(\mathrm{br}$, $\left.1 \mathrm{H},=\mathrm{CH}_{2}\right), 4.41\left(\mathrm{~m}, 1 \mathrm{H},=\mathrm{CH}_{2}\right), 3.67(\mathrm{~m}, 1 \mathrm{H}, \mathrm{CH}), 3.37\left(\mathrm{~s}, 3 \mathrm{H}, \mathrm{OCH}_{3}{ }^{\mathrm{B}}\right), 2.81(\mathrm{~s}, 3 \mathrm{H}$, $\left.\mathrm{OCH}_{3}\right), 2.30\left(\mathrm{~s}, 12 \mathrm{H}, o-\mathrm{CH}_{3}{ }^{\mathrm{Mes}}\right), 2.02\left(\mathrm{dd},{ }^{2} J_{\mathrm{HH}}=15.2 \mathrm{~Hz},{ }^{3} J_{\mathrm{HH}}=5.9 \mathrm{~Hz}, 1 \mathrm{H}, \mathrm{CH}_{2}\right)$, $1.99\left(\mathrm{~s}, 6 \mathrm{H}, p-\mathrm{CH}_{3}{ }^{\mathrm{Mes}}\right), 1.73\left(\mathrm{dd},{ }^{2} J_{\mathrm{HH}}=15.3 \mathrm{~Hz},{ }^{3} J_{\mathrm{HH}}=7.5 \mathrm{~Hz}, 1 \mathrm{H}, \mathrm{CH}_{2}\right), 1.37(\mathrm{~s}, 3 \mathrm{H}$, $\left.\mathrm{CH}_{3}\right)$.

${ }^{13} \mathbf{C}\left\{{ }^{1} \mathbf{H}\right\}$ NMR $\left(126 \mathrm{MHz}, 299 \mathrm{~K}, \mathrm{C}_{6} \mathrm{D}_{6}\right): \delta=174.4\left(\mathrm{~d},{ }^{3} J_{\mathrm{PC}}=7.8 \mathrm{~Hz}, \mathrm{C}=\mathrm{OB}\right), 166.5$ $(\mathrm{C}=\mathrm{O}), 162.5\left(\mathrm{~d},{ }^{1} J_{\mathrm{PC}}=39.8 \mathrm{~Hz},=\mathrm{CP}\right), 145.1\left(\mathrm{~d},{ }^{4} J_{\mathrm{PC}}=1.5 \mathrm{~Hz}, \mathrm{C}={ }^{\mathrm{CH} 2}\right), 144.6\left(\mathrm{~d},{ }^{2} J_{\mathrm{PC}}\right.$ $=18.0 \mathrm{~Hz}, o$-Mes $), 140.3\left(\mathrm{~d},{ }^{4} J_{\mathrm{PC}}=1.0 \mathrm{~Hz}, p\right.$-Mes $), 131.8\left(\mathrm{~d},{ }^{2} J_{\mathrm{PC}}=34.0 \mathrm{~Hz},=\mathrm{C}\right), 130.3$ $\left(\mathrm{d},{ }^{3} J_{\mathrm{PC}}=5.4 \mathrm{~Hz}, m-\mathrm{Mes}\right), 126.4\left(\mathrm{~d},{ }^{1} J_{\mathrm{PC}}=12.4 \mathrm{~Hz}, i-\mathrm{Mes}\right), 111.6\left(\mathrm{~d},{ }^{5} J_{\mathrm{PC}}=1.8 \mathrm{~Hz}\right.$, $\left.=\mathrm{CH}_{2}\right), 57.4\left(\mathrm{OCH}_{3}{ }^{\mathrm{B}}\right), 51.4\left(\mathrm{OCH}_{3}\right), 45.6\left(\mathrm{~d},{ }^{3} J_{\mathrm{PC}}=15.7 \mathrm{~Hz}, \mathrm{CH}\right), 23.2\left(\mathrm{~d},{ }^{3} J_{\mathrm{PC}}=16.7\right.$ $\left.\mathrm{Hz}, o-\mathrm{CH}_{3}{ }^{\mathrm{Mes}}\right), 23.1$ (br, $\left.\mathrm{CH}_{2}\right), 20.8\left(p-\mathrm{CH}_{3}{ }^{\mathrm{Mes}}\right), 20.4\left(\mathrm{~d},{ }^{5} \mathrm{~J}_{\mathrm{PC}}=2.3 \mathrm{~Hz}, \mathrm{CH}_{3}\right),\left[\mathrm{C}_{6} \mathrm{~F}_{5}\right.$ not listed].

${ }^{11} \mathbf{B}\left\{{ }^{1} \mathbf{H}\right\}$ NMR $\left(160 \mathrm{MHz}, 299 \mathrm{~K}, \mathrm{C}_{6} \mathrm{D}_{6}\right): \delta=4.0\left(v_{1 / 2} \sim 750 \mathrm{~Hz}\right)$.

${ }^{31} \mathbf{P}\left\{{ }^{1} \mathbf{H}\right\}$ NMR $\left(202 \mathrm{MHz}, 299 \mathrm{~K}, \mathrm{C}_{6} \mathrm{D}_{6}\right): \delta=-28.2\left(v_{1 / 2} \sim 10 \mathrm{~Hz}\right)$.

${ }^{19}$ F NMR $\left(470 \mathrm{MHz}, 299 \mathrm{~K}, \mathrm{C}_{6} \mathrm{D}_{6}\right): \delta=-134.0(\mathrm{~m}, 2 \mathrm{~F}, o),-158.1\left(\mathrm{t},{ }^{3} J_{\mathrm{FF}}=20.6 \mathrm{~Hz}, 1 \mathrm{~F}\right.$, $p),-163.9(\mathrm{~m}, 2 \mathrm{~F}, m)\left(\mathrm{C}_{6} \mathrm{~F}_{5}\right)\left[\Delta \delta^{19} \mathrm{~F}_{\mathrm{mp}}=5.8\right],-135.5(\mathrm{~m}, 2 \mathrm{~F}, o),-159.0\left(\mathrm{t},{ }^{3} J_{\mathrm{FF}}=20.7 \mathrm{~Hz}\right.$, $1 \mathrm{~F}, p),-164.4(\mathrm{~m}, 2 \mathrm{~F}, m)\left(\mathrm{C}_{6} \mathrm{~F}_{5}\right)\left[\Delta \delta^{19} \mathrm{~F}_{\mathrm{mp}}=5.4\right]$.

${ }^{1} \mathbf{H},{ }^{1} \mathbf{H}-G C O S Y\left(500 \mathrm{MHz} / 500 \mathrm{MHz}, 299 \mathrm{~K}, \mathrm{C}_{6} \mathrm{D}_{6}\right)$ [selected traces]: $\delta{ }^{1} \mathrm{H} / \delta{ }^{1} \mathrm{H}=$ 4.52, $4.41 / 1.37\left(=\mathrm{CH}_{2},=\mathrm{CH}_{2} / \mathrm{CH}_{3}\right), 3.67 / 2.02,1.73\left(\mathrm{CH} / \mathrm{CH}_{2}, \mathrm{CH}_{2}\right)$.

${ }^{1} \mathbf{H},{ }^{13} \mathbf{C}-G H S Q C\left(500 \mathrm{MHz} / 126 \mathrm{MHz}, 299 \mathrm{~K}, \mathrm{C}_{6} \mathrm{D}_{6}\right): \delta{ }^{1} \mathrm{H} / \delta{ }^{13} \mathrm{C}=6.61 / 130.3(\mathrm{~m}$ Mes), 4.52, $4.41 / 111.6\left(=\mathrm{CH}_{2}\right), 3.67 / 45.6(\mathrm{CH}), 3.37 / 57.4\left(\mathrm{OCH}_{3}{ }^{\mathrm{B}}\right), 2.81 / 51.4$ $\left(\mathrm{OCH}_{3}\right), 2.30 / 23.2\left({ }^{o-\mathrm{CH}_{3}}{ }^{\mathrm{Mes}}\right), 2.02,1.73\left(\mathrm{CH}_{2}\right), 1.99 / 20.8\left(p-\mathrm{CH}_{3}{ }^{\mathrm{Mes}}\right), 1.37 / 20.4$ $\left(\mathrm{CH}_{3}\right)$. 
${ }^{1} \mathbf{H},{ }^{13} \mathbf{C}-\mathbf{G H M B C}\left(500 \mathrm{MHz} / 126 \mathrm{MHz}, 299 \mathrm{~K}, \mathrm{C}_{6} \mathrm{D}_{6}\right)$ [selected traces]: $\delta{ }^{1} \mathrm{H} / \delta{ }^{13} \mathrm{C}=$ 6.61, $2.30 / 126.4$ ( $m$-Mes, $o$ - $\mathrm{CH}_{3}{ }^{\mathrm{Mes}} / i$-Mes $), 4.52,3.67,2.02,1.73,1.37 / 145.1\left(=\mathrm{CH}_{2}\right.$, $\left.\mathrm{CH}, \mathrm{CH}_{2}, \mathrm{CH}_{2}, \mathrm{CH}_{3} /=\mathrm{C}^{\mathrm{CH}} 2\right), 3.67,3.37 / 174.4\left(\mathrm{CH}, \mathrm{OCH}_{3}{ }^{\mathrm{B}} / \mathrm{C}=\mathrm{OB}\right), 3.67,2.02,1.73$ / $131.8\left(\mathrm{CH}, \mathrm{CH}_{2}, \mathrm{CH}_{2} /=\mathrm{C}\right), 3.67 / 162.5(\mathrm{CH} /=\mathrm{CP}), 2.81 / 166.5\left(\mathrm{OCH}_{3} / \mathrm{C}=\mathrm{O}\right), 2.30$ / $144.6\left(o-\mathrm{CH}_{3}{ }^{\mathrm{Mes}} / o-\mathrm{Mes}\right), 1.99 / 140.3\left(p-\mathrm{CH}_{3}{ }^{\mathrm{Mes}} / p\right.$-Mes $)$.

${ }^{19} \mathbf{F},{ }^{19} \mathbf{F}-G C O S Y\left(470 \mathrm{MHz} / 470 \mathrm{MHz}, 299 \mathrm{~K}, \mathrm{C}_{6} \mathrm{D}_{6}\right): \delta{ }^{19} \mathrm{~F} / \delta^{19} \mathrm{~F}=-163.9 /-134.0,-$ $158.1\left(m-\mathrm{C}_{6} \mathrm{~F}_{5}^{\mathrm{a}} / o-\mathrm{C}_{6} \mathrm{~F}_{5}{ }^{\mathrm{a}}, p-\mathrm{C}_{6} \mathrm{~F}_{5}^{\mathrm{a}}\right),-164.4 /-135.5,-159.0\left(m-\mathrm{C}_{6} \mathrm{~F}_{5} \mathrm{~b} / o-\mathrm{C}_{6} \mathrm{~F}_{5}^{\mathrm{b}}, p-\mathrm{C}_{6} \mathrm{~F}_{5}{ }^{\mathrm{b}}\right)$.

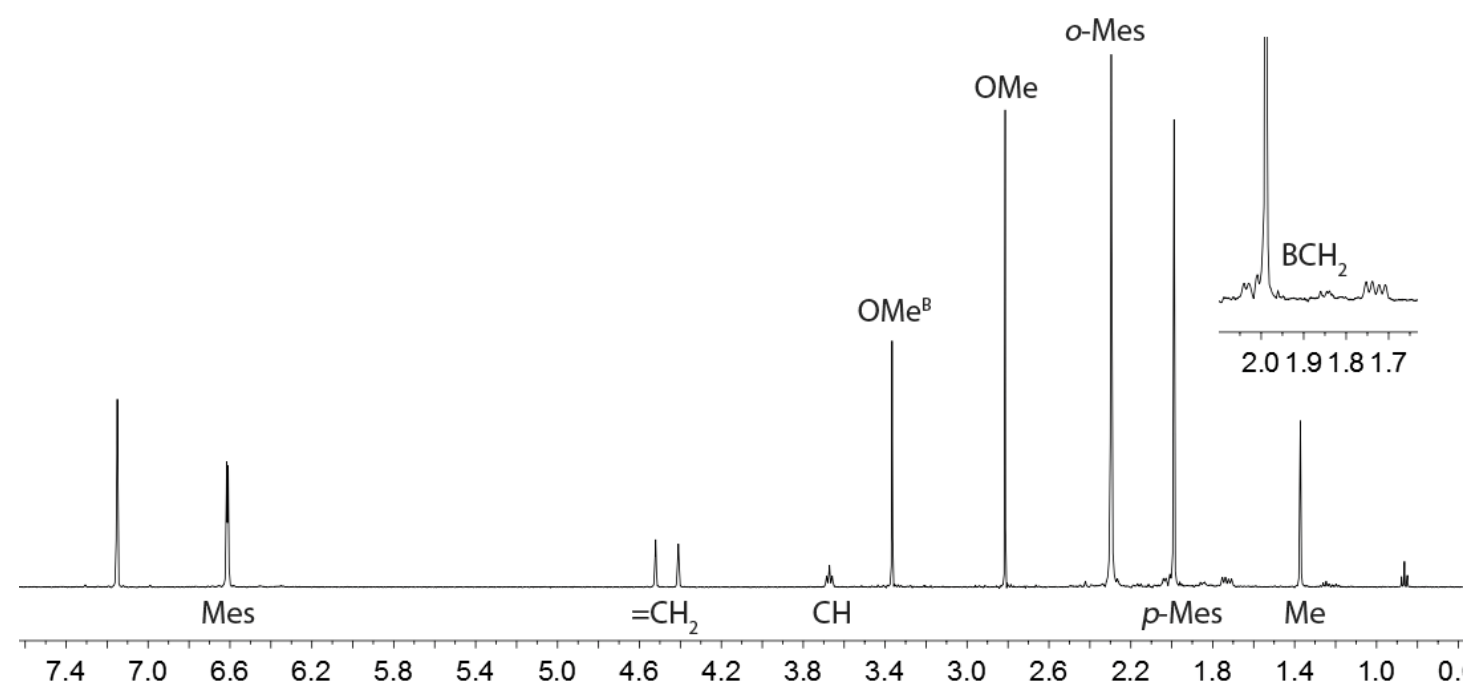

Figure S7. ${ }^{1} \mathbf{H}$ NMR $\left(500 \mathrm{MHz}, 299 \mathrm{~K}, \mathrm{C}_{6} \mathrm{D}_{6}\right)$ of compound $\mathbf{8 b}$ [admixed with pentane]

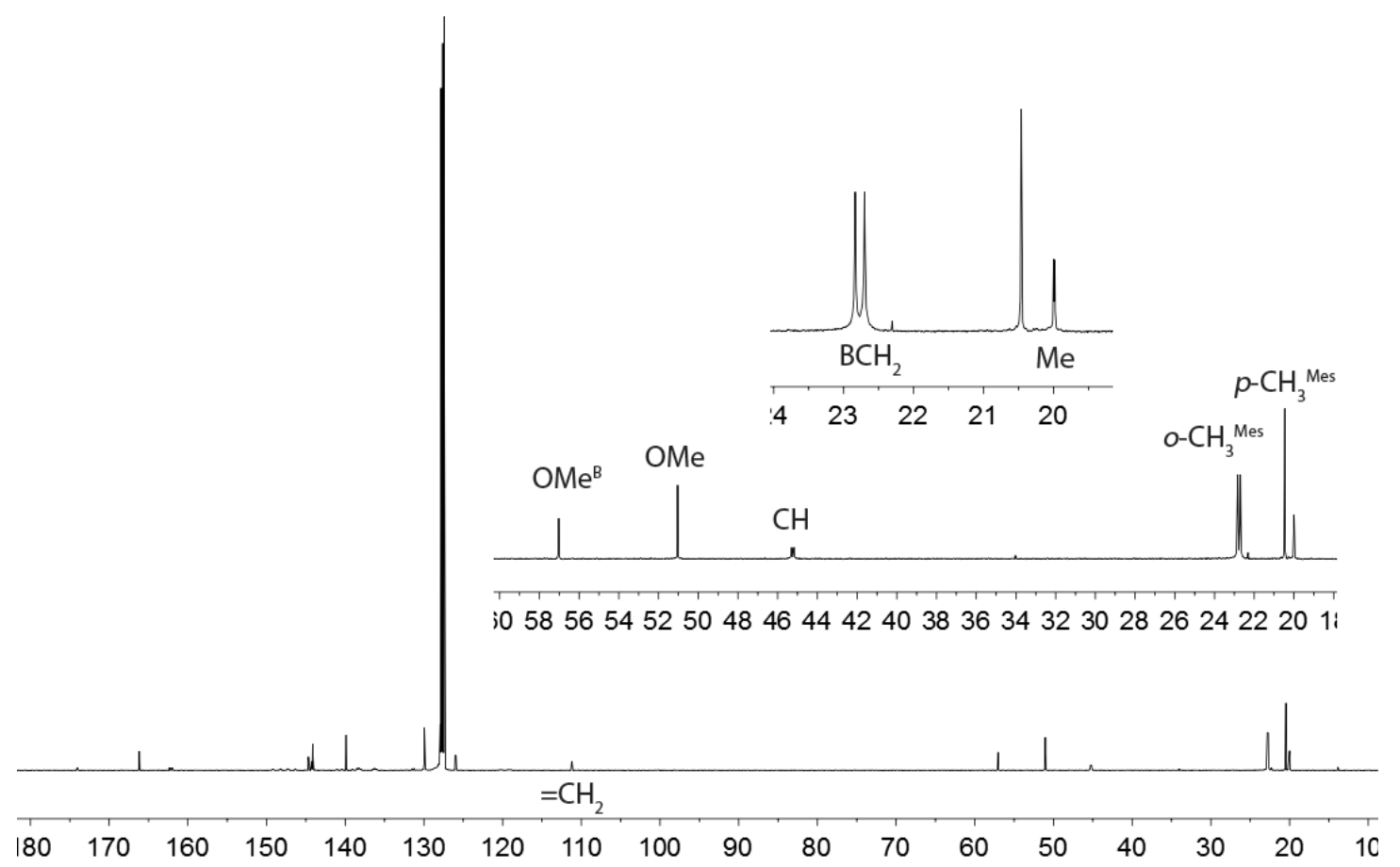

Figure S8. ${ }^{13} \mathbf{C}\left\{{ }^{1} \mathbf{H}\right\}$ NMR $\left(126 \mathrm{MHz}, 299 \mathrm{~K}, \mathrm{C}_{6} \mathrm{D}_{6}\right)$ of compound $\mathbf{8 b}$ [admixed with pentane] 


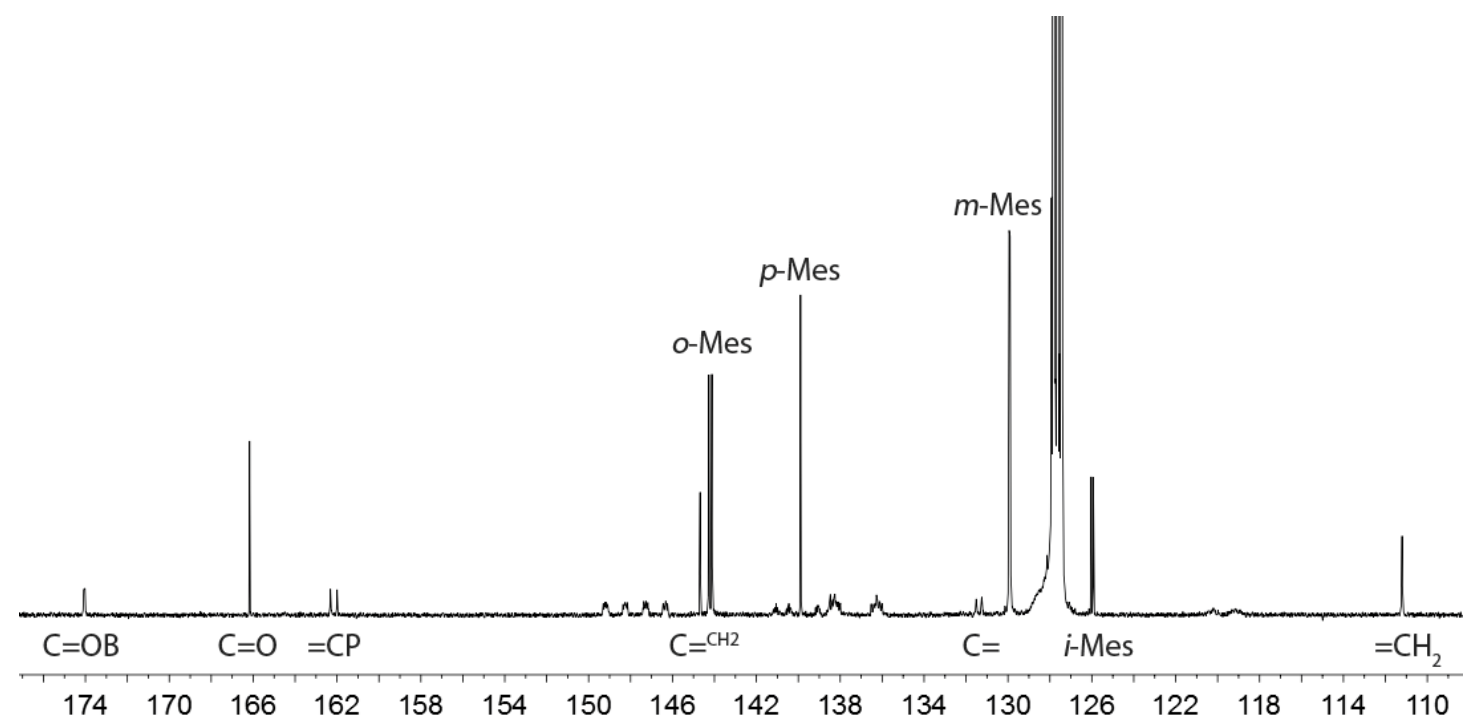

Figure S9. ${ }^{13} \mathbf{C}\left\{{ }^{1} \mathbf{H}\right\}$ NMR $\left(126 \mathrm{MHz}, 299 \mathrm{~K}, \mathrm{C}_{6} \mathrm{D}_{6}\right)$ of compound $\mathbf{8 b}$

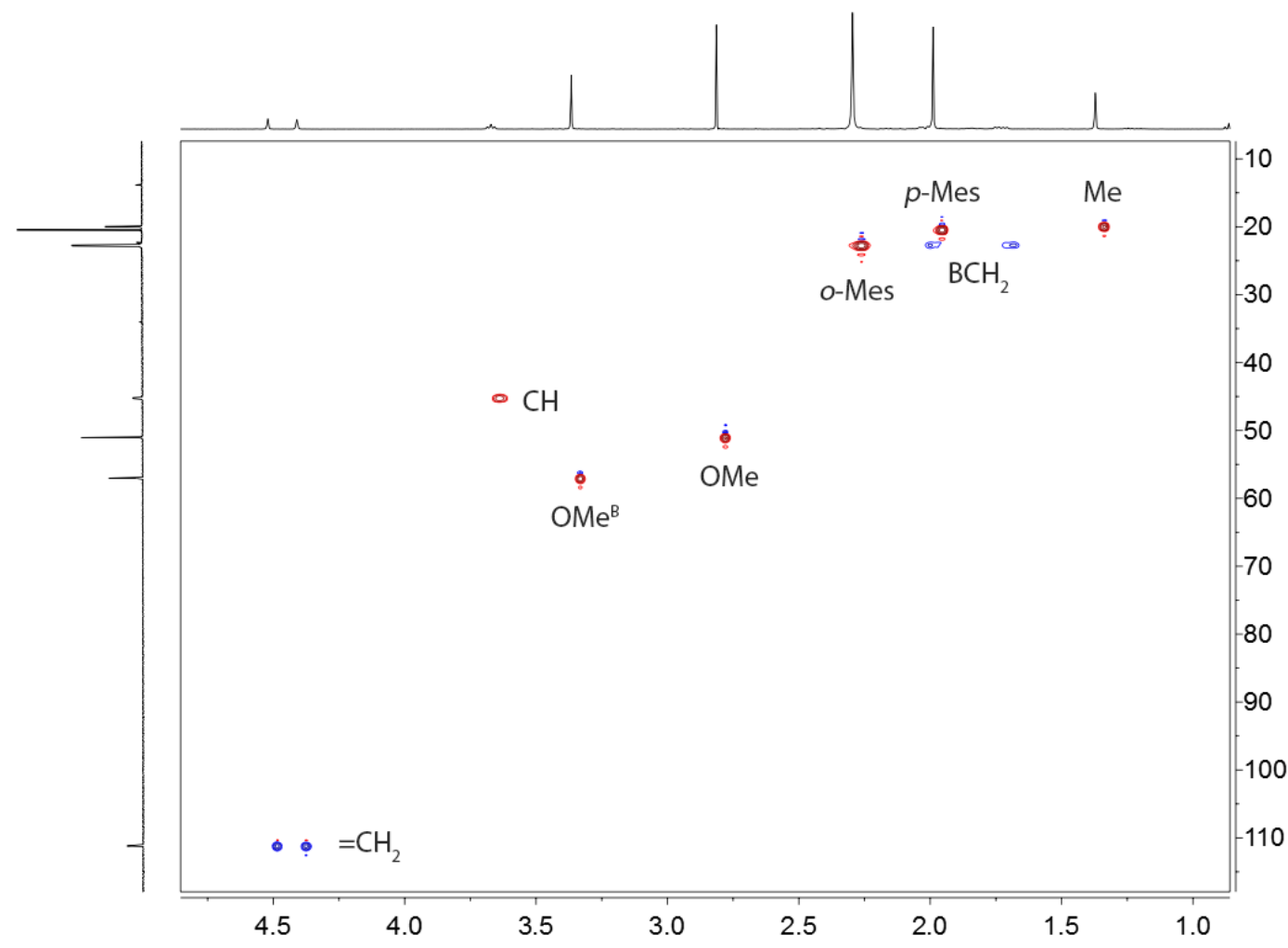

Figure S10. ${ }^{1} \mathbf{H},{ }^{13} \mathbf{C}-$ GHSQC $\left(500 \mathrm{MHz} / 126 \mathrm{MHz}, 299 \mathrm{~K}, \mathrm{C}_{6} \mathrm{D}_{6}\right)$ of compound $\mathbf{8 b}$ 


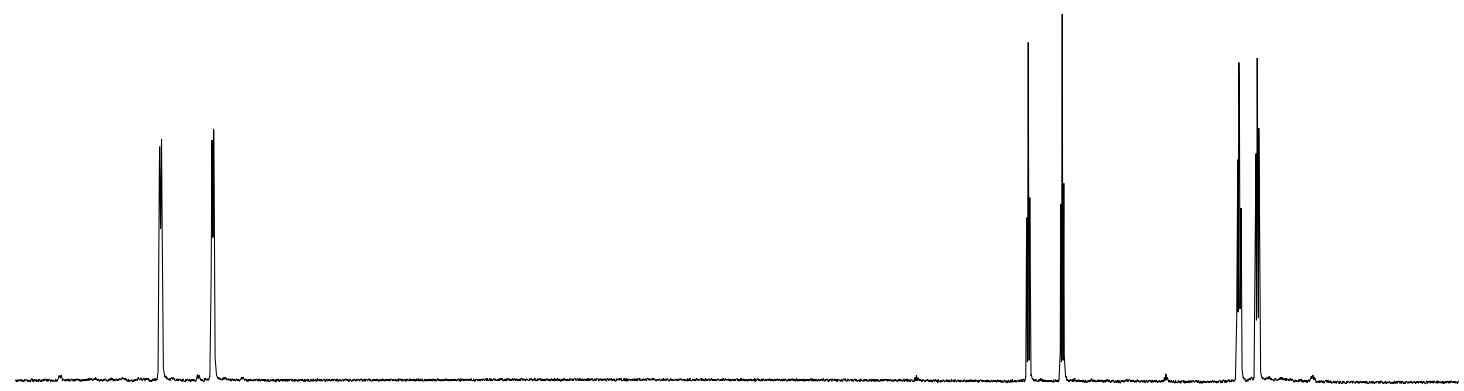

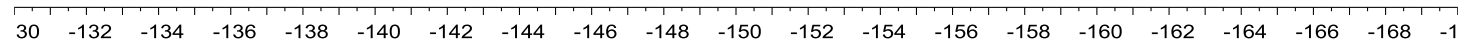

Figure S11. ${ }^{19}$ F NMR $\left(470 \mathrm{MHz}, 299 \mathrm{~K}, \mathrm{C}_{6} \mathrm{D}_{6}\right)$ of compound $\mathbf{8 b}$

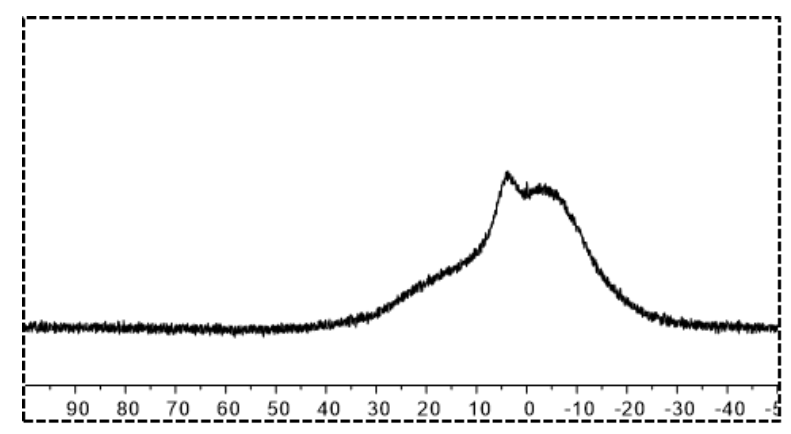

$\begin{array}{lllllllllllllllllllllllllll}300 & 280 & 260 & 240 & 220 & 200 & 180 & 160 & 140 & 120 & 100 & 80 & 60 & 40 & 20 & 0 & -20 & -40 & -60 & -80 & -10 C\end{array}$

Figure S12. ${ }^{11} \mathbf{B}\left\{{ }^{1} \mathbf{H}\right\}$ NMR $\left(160 \mathrm{MHz}, 299 \mathrm{~K}, \mathrm{C}_{6} \mathrm{D}_{6}\right)$ and ${ }^{31} \mathbf{P}\left\{{ }^{1} \mathbf{H}\right\} \mathbf{N M R}(202 \mathrm{MHz}$, $\left.299 \mathrm{~K}, \mathrm{C}_{6} \mathrm{D}_{6}\right)$ of compound $\mathbf{8 b}$

X-ray crystal structure analysis of compound 8b: A yellow prism-like specimen of $\mathrm{C}_{41} \mathrm{H}_{36} \mathrm{BF}_{10} \mathrm{O}_{4} \mathrm{P}$, approximate dimensions $0.099 \mathrm{~mm} \times 0.170 \mathrm{~mm} \times 0.257 \mathrm{~mm}$, was used for the X-ray crystallographic analysis. The X-ray intensity data were measured. A total of 511 frames were collected. The total exposure time was 4.26 hours. The frames were integrated with the Bruker SAINT software package using a narrow-frame algorithm. The integration of the data using a triclinic unit cell yielded a total of 8176 reflections to a maximum $\theta$ angle of $25.35^{\circ}(0.83 \AA$ resolution), of which 8176 were independent (average redundancy 1.000, completeness $=96.5 \%, \mathrm{R}_{\text {int }}=4.47 \%, \mathrm{R}_{\text {sig }}=6.73 \%$ ) and $5669(69.34 \%)$ were greater than $2 \sigma\left(\mathrm{F}^{2}\right)$. The final cell constants of $\underline{a}=11.3650(5) \AA$, $\underline{\mathrm{b}}=14.8236(7) \AA, \underline{\mathrm{c}}=16.1305(7) \AA, \alpha=65.606(2)^{\circ}, \beta=70.053(2)^{\circ}, \gamma=77.352(2)^{\circ}$, volume $=2316.74(19) \AA^{3}$, are based upon the refinement of the XYZ-centroids of 5798 reflections above $20 \sigma(\mathrm{I})$ with $4.660^{\circ}<2 \theta<54.83^{\circ}$. Data were corrected for absorption effects using the multi-scan method (SADABS). The ratio of minimum to maximum apparent transmission was 0.931 . The calculated minimum and maximum transmission 
coefficients (based on crystal size) are 0.9660 and 0.9870 . The structure was solved and refined using the Bruker SHELXTL Software Package, using the space group P-1, with $\mathrm{Z}=2$ for the formula unit, $\mathrm{C}_{41} \mathrm{H}_{36} \mathrm{BF}_{10} \mathrm{O}_{4} \mathrm{P}$. The final anisotropic full-matrix leastsquares refinement on $\mathrm{F}^{2}$ with 523 variables converged at $\mathrm{R} 1=5.72 \%$, for the observed data and wR2 $=16.48 \%$ for all data. The goodness-of-fit was 1.048 . The largest peak in the final difference electron density synthesis was $0.502 \mathrm{e}^{-} / \AA^{3}$ and the largest hole was $-0.274 \mathrm{e}^{-} / \AA^{3}$ with an RMS deviation of $0.067 \mathrm{e}^{-} / \AA^{3}$. On the basis of the final model, the calculated density was $1.182 \mathrm{~g} / \mathrm{cm}^{3}$ and $\mathrm{F}(000), 848 \mathrm{e}^{-}$.

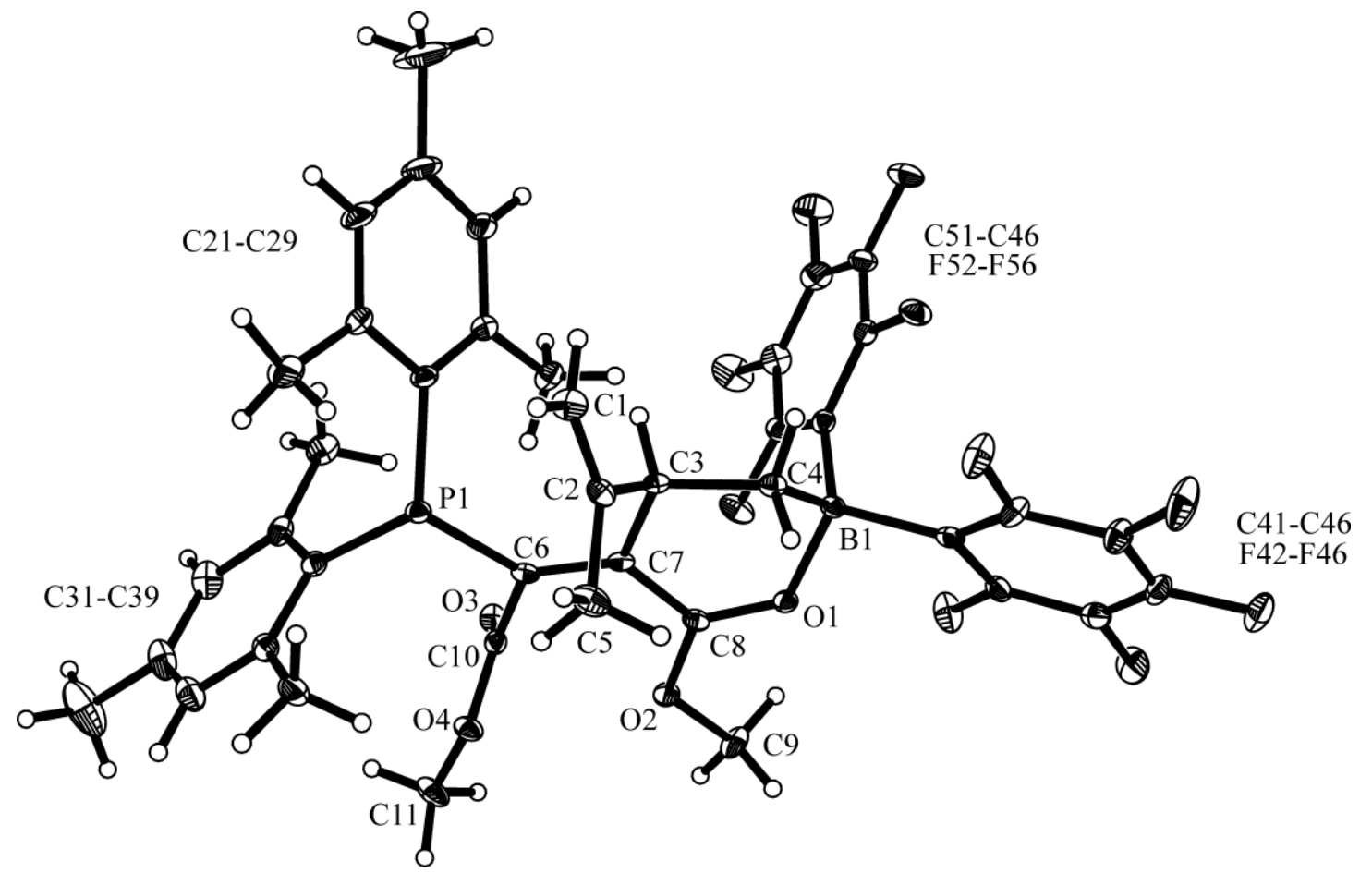

Figure S13. 


\section{Synthesis of compound 8c}
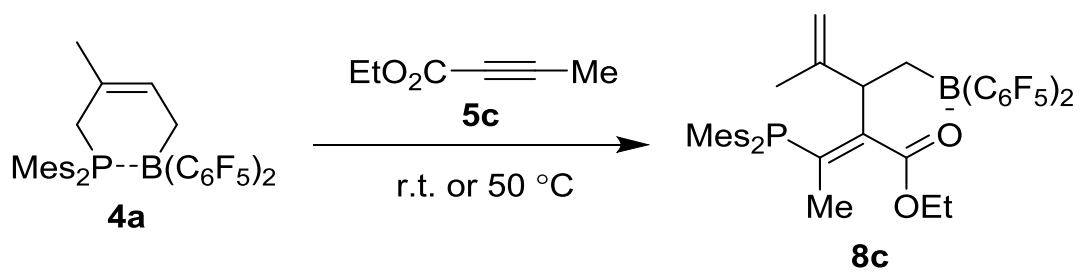

Scheme S3

A solution of compounds $\mathbf{4 a}(102.3 \mathrm{mg}, 0.15 \mathrm{mmol})$ and $\mathbf{5 c}(16.8 \mathrm{mg}, 0.15 \mathrm{mmol})$ in dichloromethane $\left(3 \mathrm{~mL}\right.$ ) was heated at $50{ }^{\circ} \mathrm{C}$ for 1 day (or alternatively stirred at room temperature for 7 days). Then all volatiles were removed in vacuo and compound $8 \mathbf{c}$ (109.1 mg, $92 \%$ ) was obtained as an orange solid. Decomp. $173{ }^{\circ} \mathrm{C}$. Anal. Calc. for $\mathrm{C}_{41} \mathrm{H}_{38} \mathrm{BF}_{10} \mathrm{O}_{2} \mathrm{P}: \mathrm{C}: 61.98 ; \mathrm{H}: 4.82$. Found: C:62.39; H:4.71.

${ }^{1} \mathbf{H}$ NMR $\left(600 \mathrm{MHz}, 299 \mathrm{~K}, \mathrm{C}_{6} \mathrm{D}_{6}\right): \delta=6.59\left(\mathrm{dm},{ }^{4} J_{\mathrm{PH}}=3.4 \mathrm{~Hz}, 4 \mathrm{H}, m-\mathrm{Mes}\right), 4.63(\mathrm{br}$, $\left.1 \mathrm{H},=\mathrm{CH}_{2}\right), 4.59\left(\mathrm{~m}, 1 \mathrm{H},=\mathrm{CH}_{2}\right), 4.06\left(\mathrm{q},{ }^{3} J_{\mathrm{HH}}=7.2 \mathrm{~Hz}, 2 \mathrm{H}, \mathrm{OEt}\right), 3.44(\mathrm{~m}, 1 \mathrm{H}, \mathrm{CH})$, $2.10\left(\mathrm{dd},{ }^{2} J_{\mathrm{HH}}=15.1 \mathrm{~Hz},{ }^{3} J_{\mathrm{HH}}=6.3 \mathrm{~Hz}, 1 \mathrm{H}, \mathrm{CH}_{2}\right), 2.01\left(\mathrm{~m}, 12 \mathrm{H}, o-\mathrm{CH}_{3}{ }^{\mathrm{Mes}}\right), 2.01(\mathrm{~s}$, $\left.6 \mathrm{H}, p-\mathrm{CH}_{3}{ }^{\mathrm{Mes}}\right), 1.70\left(\mathrm{~d},{ }^{3} J_{\mathrm{PH}}=5.7 \mathrm{~Hz}, 3 \mathrm{H},{ }^{\mathrm{P}} \mathrm{CH}_{3}\right), 1.61\left(\mathrm{dd},{ }^{2} J_{\mathrm{HH}}=15.1 \mathrm{~Hz},{ }^{3} J_{\mathrm{HH}}=8.9\right.$ $\left.\mathrm{Hz}, 1 \mathrm{H}, \mathrm{CH}_{2}\right), 1.58\left(\mathrm{~m}, 3 \mathrm{H}, \mathrm{CH}_{3}\right), 0.40\left(\mathrm{t},{ }^{3} J_{\mathrm{HH}}=7.2 \mathrm{~Hz}, 3 \mathrm{H}, \mathrm{OEt}\right)$.

${ }^{13} \mathbf{C}\left\{{ }^{1} \mathbf{H}\right\}$ NMR $\left(151 \mathrm{MHz}, 299 \mathrm{~K}, \mathrm{C}_{6} \mathrm{D}_{6}\right): \delta=175.7(\mathrm{C}=\mathrm{OB}), 170.3\left(\mathrm{~d},{ }^{1} J_{\mathrm{PC}}=42.9 \mathrm{~Hz}\right.$, $=\mathrm{CP}), 148.1\left(\mathrm{~d},{ }^{4} J_{\mathrm{PC}}=3.6 \mathrm{~Hz}, \mathrm{C}={ }^{\mathrm{CH} 2}\right), 142.6\left(\mathrm{~d},{ }^{3} J_{\mathrm{PC}}=17.0 \mathrm{~Hz}, o-\mathrm{Mes}\right), 139.1(p-\mathrm{Mes})$, $134.4\left(\mathrm{~d},{ }^{2} J_{\mathrm{PC}}=24.3 \mathrm{~Hz},=\mathrm{C}\right), 131.1\left(\mathrm{~d},{ }^{1} J_{\mathrm{PC}}=12.5 \mathrm{~Hz}, i-\mathrm{Mes}\right), 130.2\left(\mathrm{~d},{ }^{3} J_{\mathrm{PC}}=4.6 \mathrm{~Hz}\right.$, $m$-Mes), $110.6\left(=\mathrm{CH}_{2}\right), 68.2(\mathrm{OEt}), 46.1(\mathrm{CH}), 24.0\left(\mathrm{br}, \mathrm{CH}_{2}\right), 23.1\left(\mathrm{br} \mathrm{d},{ }^{3} J_{\mathrm{PC}}=16.3\right.$ $\left.\mathrm{Hz}, o-\mathrm{CH}_{3}{ }^{\mathrm{Mes}}\right), 23.0\left(\mathrm{~d},{ }^{2} J_{\mathrm{PC}}=2.0 \mathrm{~Hz},{ }^{\mathrm{P}} \mathrm{CH}_{3}\right), 20.79\left(\mathrm{CH}_{3}\right), 20.77\left(p-\mathrm{CH}_{3}{ }^{\mathrm{Mes}}\right), 12.5(\mathrm{OEt})$, $\left[\mathrm{C}_{6} \mathrm{~F}_{5}\right.$ not listed].

${ }^{11} \mathbf{B}\left\{{ }^{1} \mathbf{H}\right\}$ NMR $\left(160 \mathrm{MHz}, 299 \mathrm{~K}, \mathrm{C}_{6} \mathrm{D}_{6}\right): \delta=2.8\left(v_{1 / 2} \sim 500 \mathrm{~Hz}\right)$.

${ }^{10} \mathbf{B}\left\{{ }^{1} \mathbf{H}\right\}$ NMR $\left(54 \mathrm{MHz}, 299 \mathrm{~K}, \mathrm{C}_{6} \mathrm{D}_{6}\right): \delta=2.8\left(v_{1 / 2} \sim 550 \mathrm{~Hz}\right)$.

${ }^{31} \mathbf{P}\left\{{ }^{1} \mathbf{H}\right\}$ NMR $\left(243 \mathrm{MHz}, 299 \mathrm{~K}, \mathrm{C}_{6} \mathrm{D}_{6}\right): \delta=-11.0(\mathrm{~m})$.

${ }^{19}$ F NMR $\left(564 \mathrm{MHz}, 299 \mathrm{~K}, \mathrm{C}_{6} \mathrm{D}_{6}\right): \delta=-134.1(\mathrm{~m}, 2 \mathrm{~F}, o),-158.3\left(\mathrm{t},{ }^{3} J_{\mathrm{FF}}=20.6 \mathrm{~Hz}, 1 \mathrm{~F}\right.$, $p),-163.8(\mathrm{~m}, 2 \mathrm{~F}, m)\left(\mathrm{C}_{6} \mathrm{~F}_{5}\right)\left[\Delta \delta^{19} \mathrm{~F}_{\mathrm{mp}}=5.5\right],-135.5(\mathrm{~m}, 2 \mathrm{~F}, o),-159.2\left(\mathrm{t},{ }^{3} J_{\mathrm{FF}}=20.5 \mathrm{~Hz}\right.$, $1 \mathrm{~F}, p),-164.5(\mathrm{~m}, 2 \mathrm{~F}, m)\left(\mathrm{C}_{6} \mathrm{~F}_{5}\right)\left[\Delta \delta^{19} \mathrm{~F}_{\mathrm{mp}}=5.3\right]$.

${ }^{1} \mathbf{H},{ }^{1} \mathrm{H}$-GCOSY $\left(600 \mathrm{MHz} / 600 \mathrm{MHz}, 299 \mathrm{~K}, \mathrm{C}_{6} \mathrm{D}_{6}\right)$ [selected traces]: $\delta{ }^{1} \mathrm{H} / \delta{ }^{1} \mathrm{H}=$ 4.63, $4.60 / 1.58\left(=\mathrm{CH}_{2},=\mathrm{CH}_{2} / \mathrm{CH}_{3}\right), 3.44 / 2.10,1.61\left(\mathrm{CH} / \mathrm{CH}_{2}, \mathrm{CH}_{2}\right)$.

${ }^{1} \mathbf{H},{ }^{13} \mathbf{C}-G H S Q C\left(600 \mathrm{MHz} / 151 \mathrm{MHz}, 299 \mathrm{~K}, \mathrm{C}_{6} \mathrm{D}_{6}\right): \delta{ }^{1} \mathrm{H} / \delta{ }^{13} \mathrm{C}=6.59 / 130.2(\mathrm{~m}-$ Mes), 4.63, 4.59 / $110.6\left(=\mathrm{CH}_{2}\right), 4.06 / 68.2(\mathrm{OEt}), 3.44$ / $46.1(\mathrm{CH}), 2.10,1.61 / 24.0$ $\left(\mathrm{CH}_{2}\right), 2.01 / 23.1\left(o-\mathrm{CH}_{3}{ }^{\mathrm{Mes}}\right), 2.01 / 20.77\left(p-\mathrm{CH}_{3}{ }^{\mathrm{Mes}}\right), 1.70 / 23.0\left({ }^{\mathrm{P}} \mathrm{CH}_{3}\right), 1.58 / 20.79$ $\left(\mathrm{CH}_{3}\right), 0.40 / 12.5(\mathrm{OEt})$.

${ }^{\mathbf{1}} \mathbf{H},{ }^{13} \mathbf{C}-\mathbf{G H M B C}\left(600 \mathrm{MHz} / 151 \mathrm{MHz}, 299 \mathrm{~K}, \mathrm{C}_{6} \mathrm{D}_{6}\right)$ [selected traces]: $\delta{ }^{1} \mathrm{H} / \delta^{13} \mathrm{C}=$ 6.59, $2.01 / 131.1$ ( $m$-Mes, $o$ - $\mathrm{CH}_{3}{ }^{\mathrm{Mes}} / i$-Mes), 4.63, 4.59, 3.44, 2.10, 1.61, $1.58 / 148.1$ $\left(=\mathrm{CH}_{2},=\mathrm{CH}_{2}, \mathrm{CH}, \mathrm{CH}_{2}, \mathrm{CH}_{2}, \mathrm{CH}_{3} / \mathrm{C}={ }^{\mathrm{CH}} 2\right), 4.06,3.44 / 175.7(\mathrm{OEt}, \mathrm{CH} / \mathrm{C}=\mathrm{OB}), 3.44$, 
2.10, 1.70, $1.61 / 134.4\left(\mathrm{CH}, \mathrm{CH}_{2},{ }^{\mathrm{P}} \mathrm{CH}_{3}, \mathrm{CH}_{2} /=\mathrm{CCH}\right), 3.44,1.70 / 170.3\left(\mathrm{CH},{ }^{\mathrm{P}} \mathrm{CH}_{3} /\right.$ $=\mathrm{CP}), 2.01 / 139.1$ ( $p-\mathrm{CH}_{3}{ }^{\mathrm{Mes}} / p$-Mes), $2.01 / 142.6\left(o-\mathrm{CH}_{3}{ }^{\mathrm{Mes}} / o-\mathrm{Mes}\right)$.

${ }^{19} \mathrm{~F},{ }^{19} \mathrm{~F}-G C O S Y\left(564 \mathrm{MHz} / 564 \mathrm{MHz}, 299 \mathrm{~K}, \mathrm{C}_{6} \mathrm{D}_{6}\right): \delta{ }^{19} \mathrm{~F} / \delta{ }^{19} \mathrm{~F}=-163.8 /-134.1,-$ $158.3\left(m-\mathrm{C}_{6} \mathrm{~F}_{5}^{\mathrm{a}} / o-\mathrm{C}_{6} \mathrm{~F}_{5}{ }^{\mathrm{a}}, p-\mathrm{C}_{6} \mathrm{~F}_{5}^{\mathrm{a}}\right),-164.5 /-135.5,-159.2\left(m-\mathrm{C}_{6} \mathrm{~F}_{5} \mathrm{~b} / o-\mathrm{C}_{6} \mathrm{~F}_{5}^{\mathrm{b}}, p-\mathrm{C}_{6} \mathrm{~F}_{5}{ }^{\mathrm{b}}\right)$.

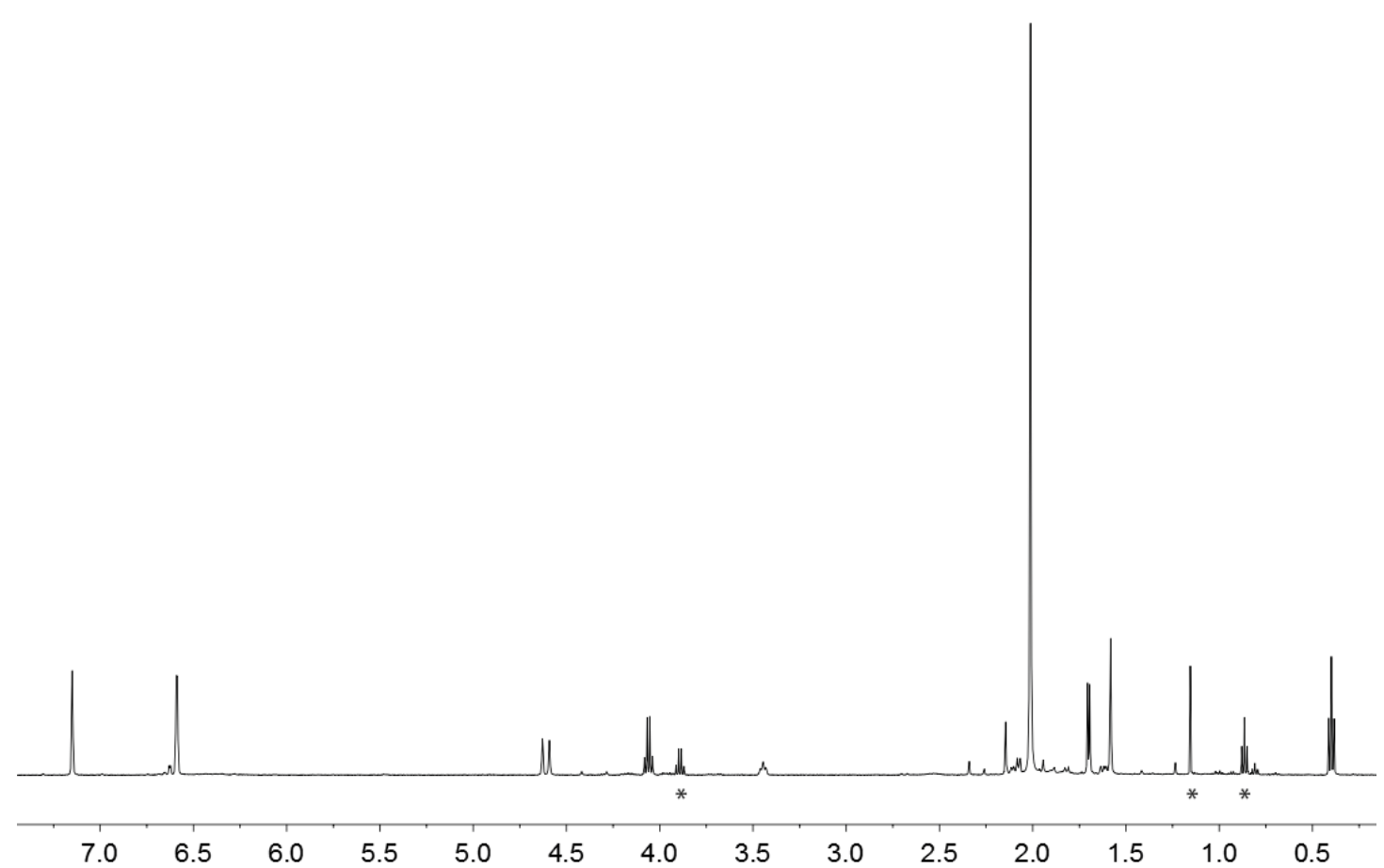

Figure S14. ${ }^{1} \mathbf{H}$ NMR $\left(500 \mathrm{MHz}, 299 \mathrm{~K}, \mathrm{C}_{6} \mathrm{D}_{6}\right)$ of compound $\mathbf{8 c}$ [admixed with $\left.\mathrm{EtO}_{2} \mathrm{C}-\mathrm{C} \equiv \mathrm{C}-\mathrm{Me}(*)\right]$

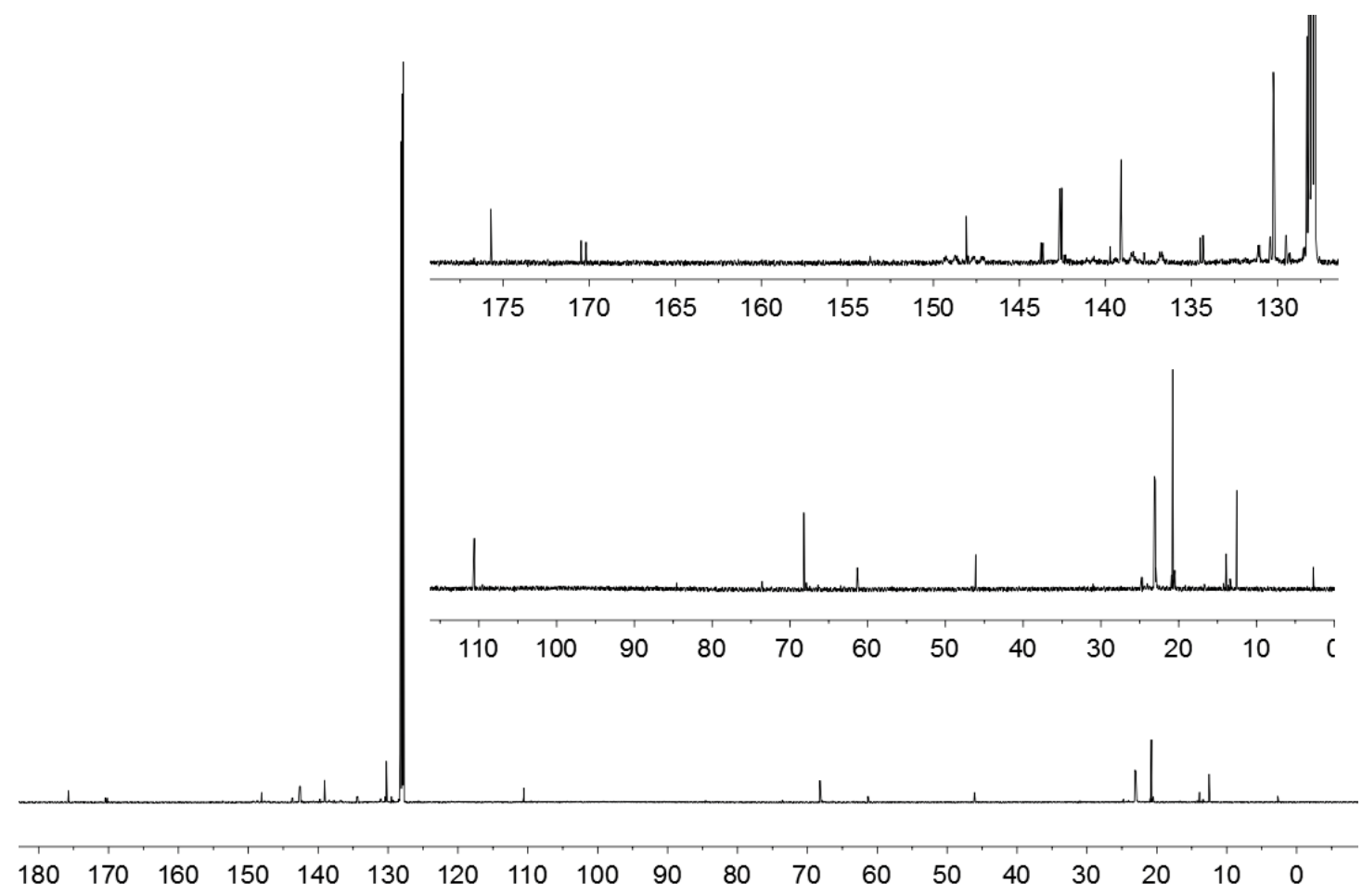

Figure S15. ${ }^{13} \mathbf{C}\left\{{ }^{1} \mathbf{H}\right\}$ NMR $\left(126 \mathrm{MHz}, 299 \mathrm{~K}, \mathrm{C}_{6} \mathrm{D}_{6}\right)$ of compound $8 \mathbf{c}$ [admixed with $\mathrm{EtO}_{2} \mathrm{C}-\mathrm{C} \equiv \mathrm{C}-\mathrm{Me}$ 

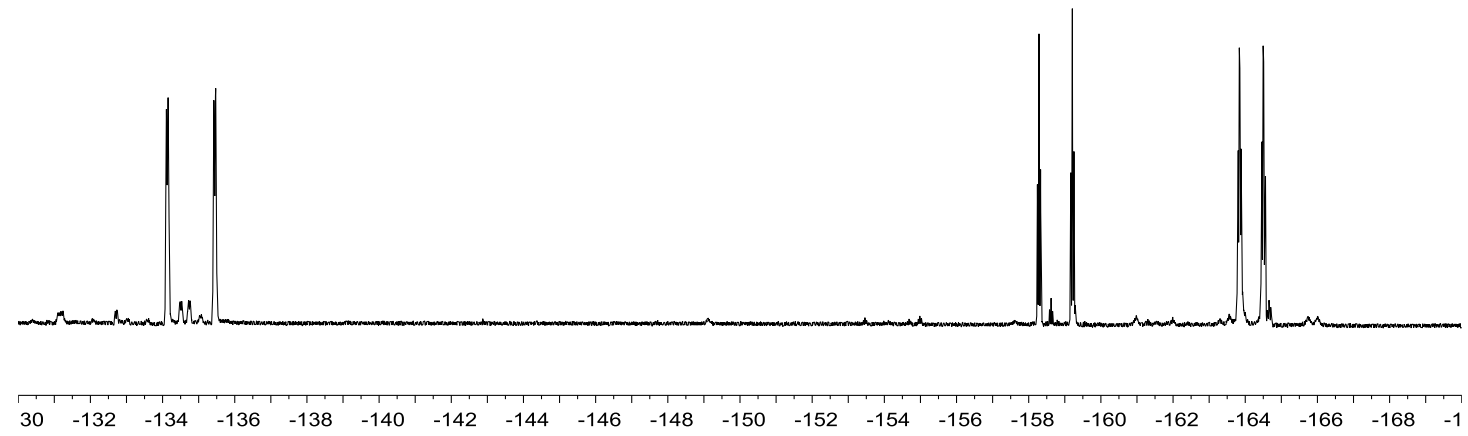

Figure S16. ${ }^{19}$ F NMR $\left(470 \mathrm{MHz}, 299 \mathrm{~K}, \mathrm{C}_{6} \mathrm{D}_{6}\right)$ of compound $8 \mathrm{c}$

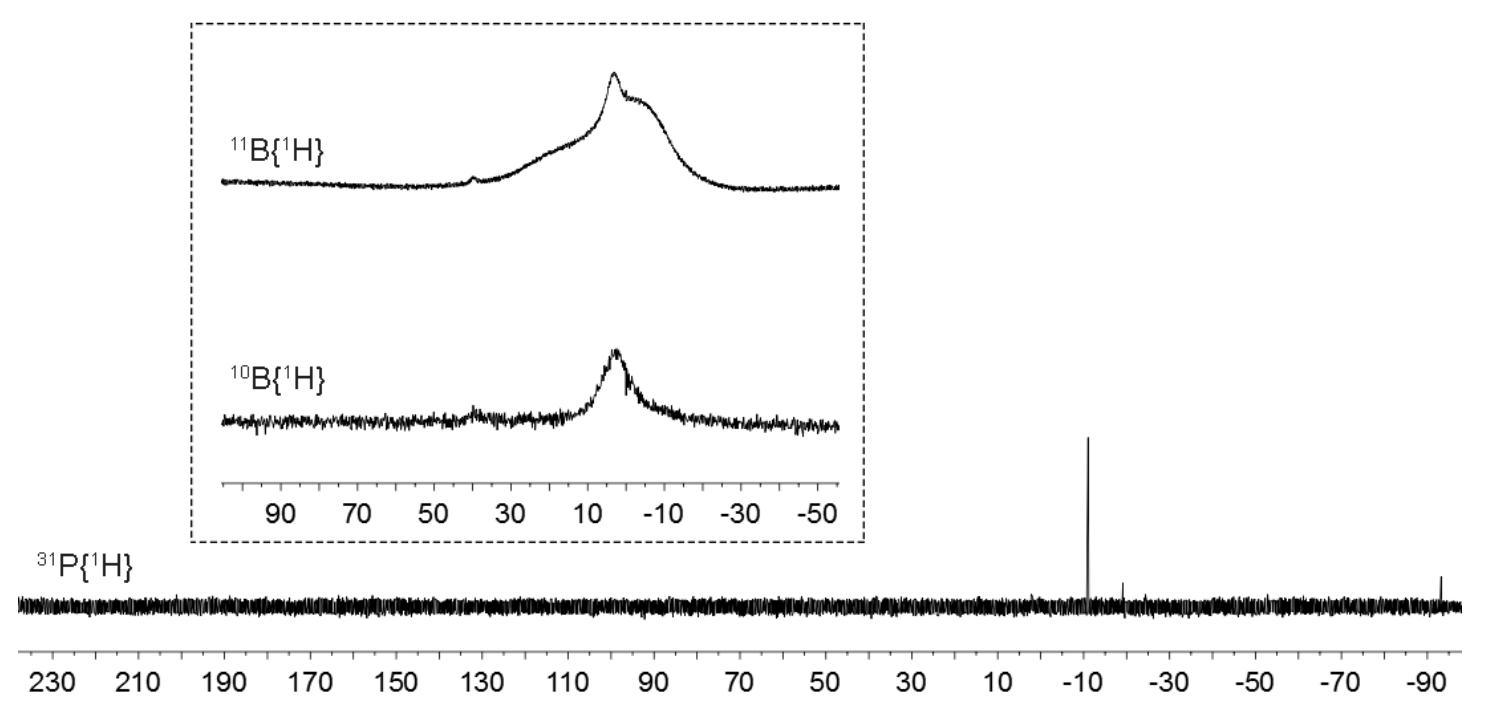

Figure S17. ${ }^{11} \mathbf{B}\left\{{ }^{1} \mathbf{H}\right\}$ NMR $\left(160 \mathrm{MHz}, 299 \mathrm{~K}, \mathrm{C}_{6} \mathrm{D}_{6}\right),{ }^{10} \mathbf{B}\left\{{ }^{\mathbf{1}} \mathbf{H}\right\}$ NMR $(54 \mathrm{MHz}, 299 \mathrm{~K}$, $\left.\mathrm{C}_{6} \mathrm{D}_{6}\right)$ and ${ }^{31} \mathbf{P}\left\{{ }^{1} \mathbf{H}\right\} \mathbf{N M R}\left(243 \mathrm{MHz}, 299 \mathrm{~K}, \mathrm{C}_{6} \mathrm{D}_{6}\right)$ of compound $\mathbf{8 c}$ 


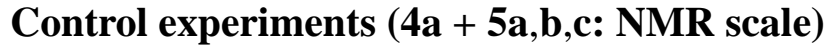

Reactions of compound $\mathbf{4 a}$ with the alkynes $\mathbf{5 a}, \mathbf{5 b}$ and $\mathbf{5 c}$, respectively, monitored by NMR spectroscopy

Alkyne $5 \mathbf{a}(0.03 \mathrm{mmol}, 2.5 \mathrm{mg})$ [5b $(0.03 \mathrm{mmol}, 4.3 \mathrm{mg}), \mathbf{5 c}(0.03 \mathrm{mmol}, 3.4 \mathrm{mg})]$ was added to a solution of compound $4 \mathbf{a}(0.03 \mathrm{mmol}, 20.5 \mathrm{mg})$ in $\mathrm{C}_{6} \mathrm{D}_{6}(0.5 \mathrm{~mL})$. Then the reaction mixture was transferred to a NMR tube and monitored by NMR spectroscopy.

Comment 1: NMR spectra showed that the reactions of compound 4a with the alkynes $\mathbf{5 a}$ or $\mathbf{5 b}$ were finished in less than $10 \mathrm{~min}$. after mixing the components at r.t. to give compounds $\mathbf{8 a}$ and $\mathbf{8 b}$, respectively, in quantitative yields.

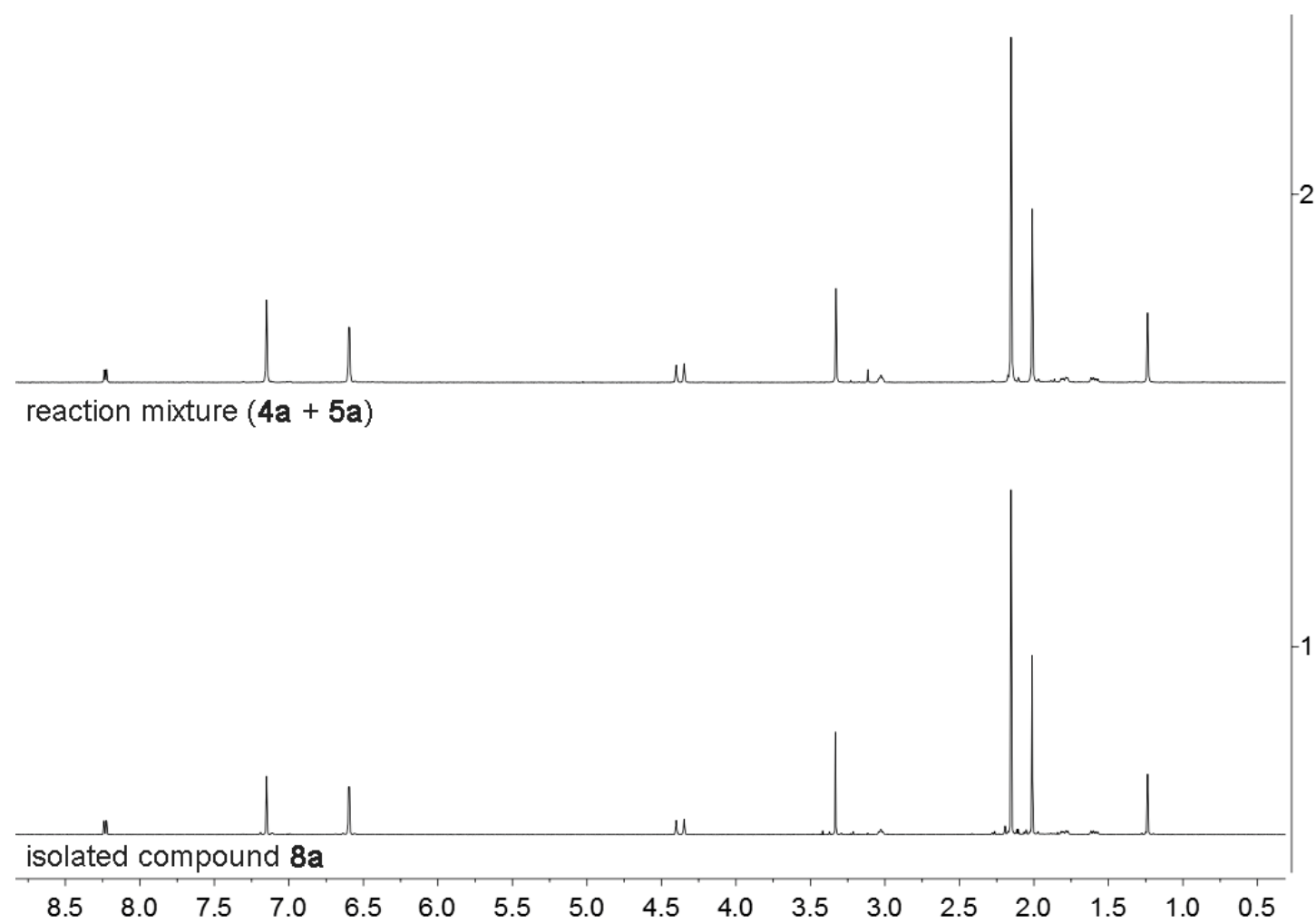

Figure S18. ${ }^{1} \mathbf{H}$ NMR $\left(500 \mathrm{MHz}, 299 \mathrm{~K}, \mathrm{C}_{6} \mathrm{D}_{6}\right)$ spectra of (1) isolated compound 8a and (2) the reaction mixture of the reaction of compounds $\mathbf{4 a}$ with $\mathbf{5 a}$ 


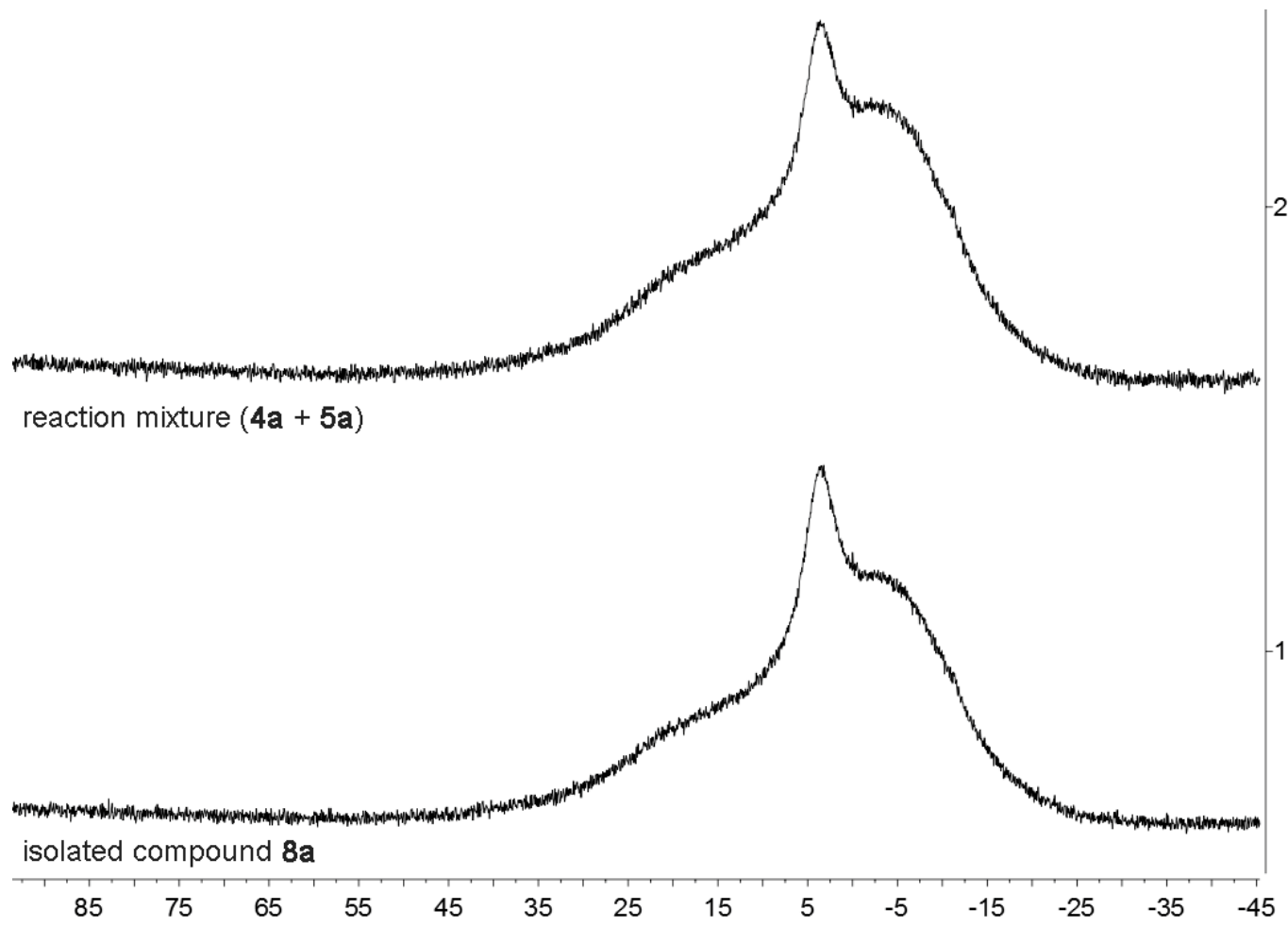

Figure S19. ${ }^{11} \mathbf{B}\left\{{ }^{1} \mathbf{H}\right\}$ NMR $\left(160 \mathrm{MHz}, 299 \mathrm{~K}, \mathrm{C}_{6} \mathrm{D}_{6}\right)$ spectra of (1) isolated compound $\mathbf{8 a}$ and (2) the reaction mixture of the reaction of compounds $4 \mathbf{a}$ with $\mathbf{5 a}$

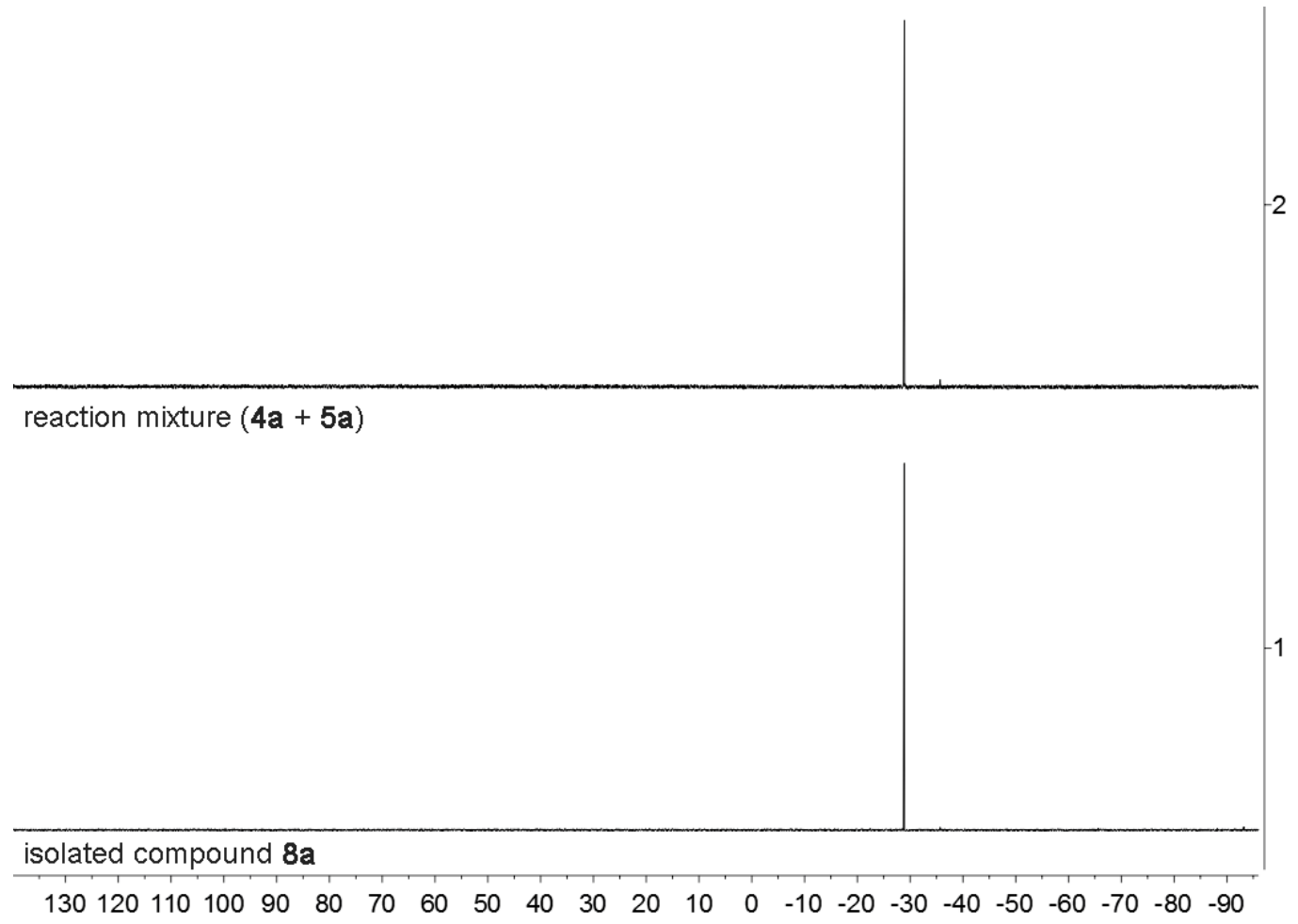

Figure S20. ${ }^{31} \mathbf{P}\left\{{ }^{1} \mathbf{H}\right\}$ NMR $\left(202 \mathrm{MHz}, 299 \mathrm{~K}, \mathrm{C}_{6} \mathrm{D}_{6}\right)$ spectra of (1) isolated compound $\mathbf{8 a}$ and (2) the reaction mixture of the reaction of compounds $\mathbf{4 a}$ with $\mathbf{5 a}$ 


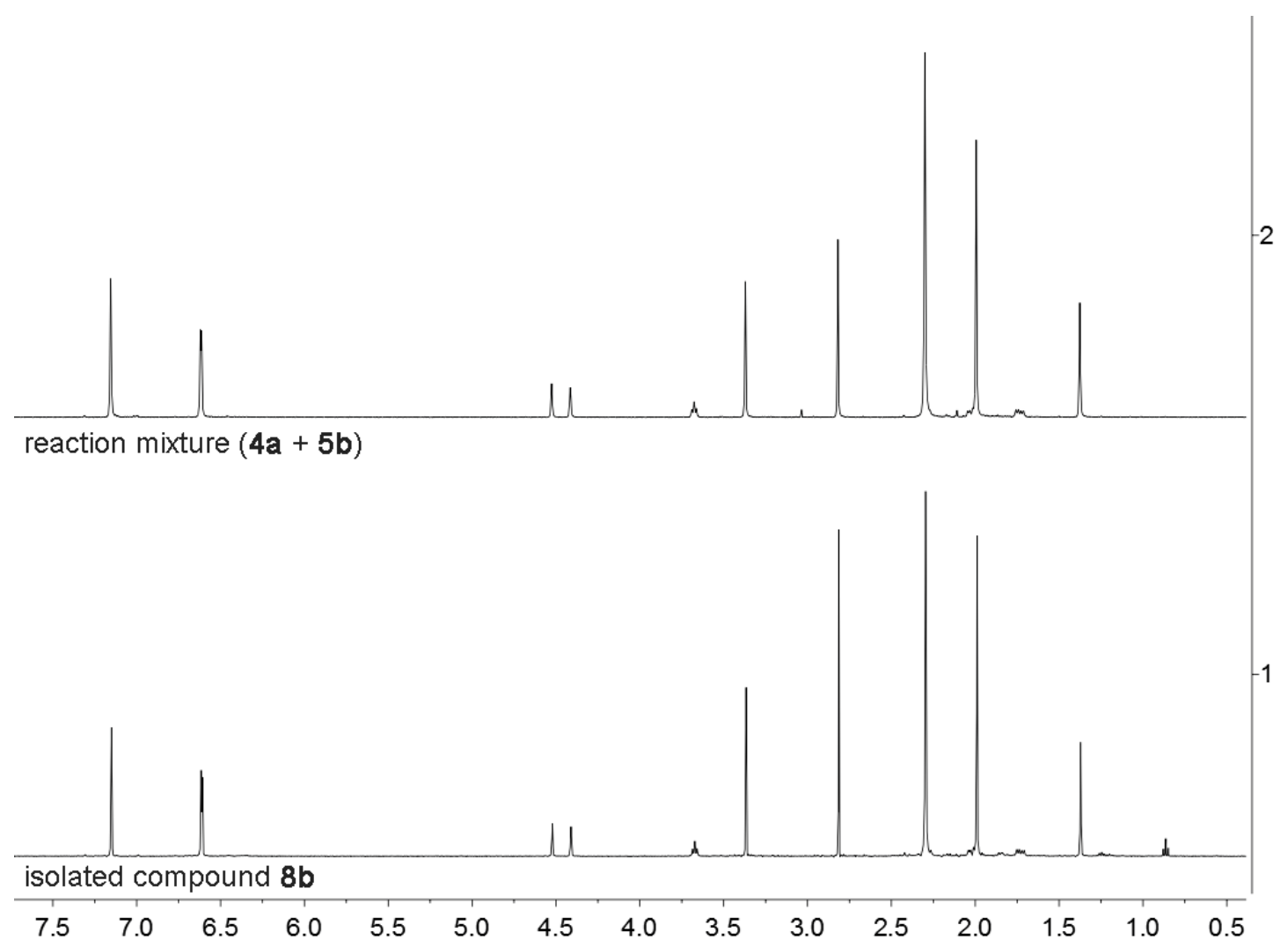

Figure S21. ${ }^{1} \mathbf{H}$ NMR $\left(500 \mathrm{MHz}, 299 \mathrm{~K}, \mathrm{C}_{6} \mathrm{D}_{6}\right)$ spectra of (1) isolated compound $\mathbf{8 b}$ and (2) the reaction mixture of the reaction of compounds $\mathbf{4 a}$ with $\mathbf{5 b}$

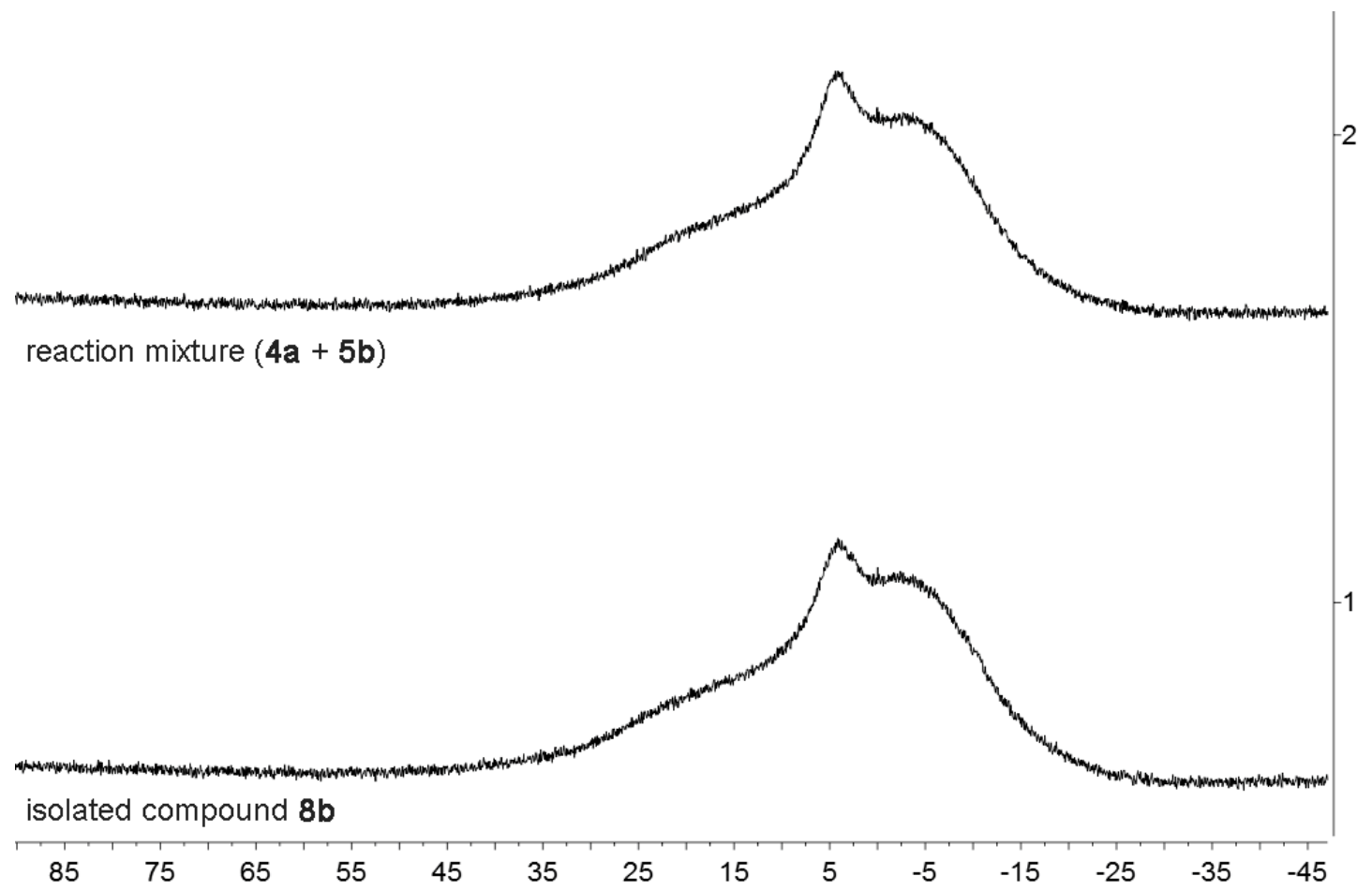

Figure S22. ${ }^{11} \mathbf{B}\left\{{ }^{1} \mathbf{H}\right\}$ NMR $\left(160 \mathrm{MHz}, 299 \mathrm{~K}, \mathrm{C}_{6} \mathrm{D}_{6}\right)$ spectra of (1) isolated compound $\mathbf{8 b}$ and (2) the reaction mixture of the reaction of compounds $\mathbf{4 a}$ with $\mathbf{5 b}$ 


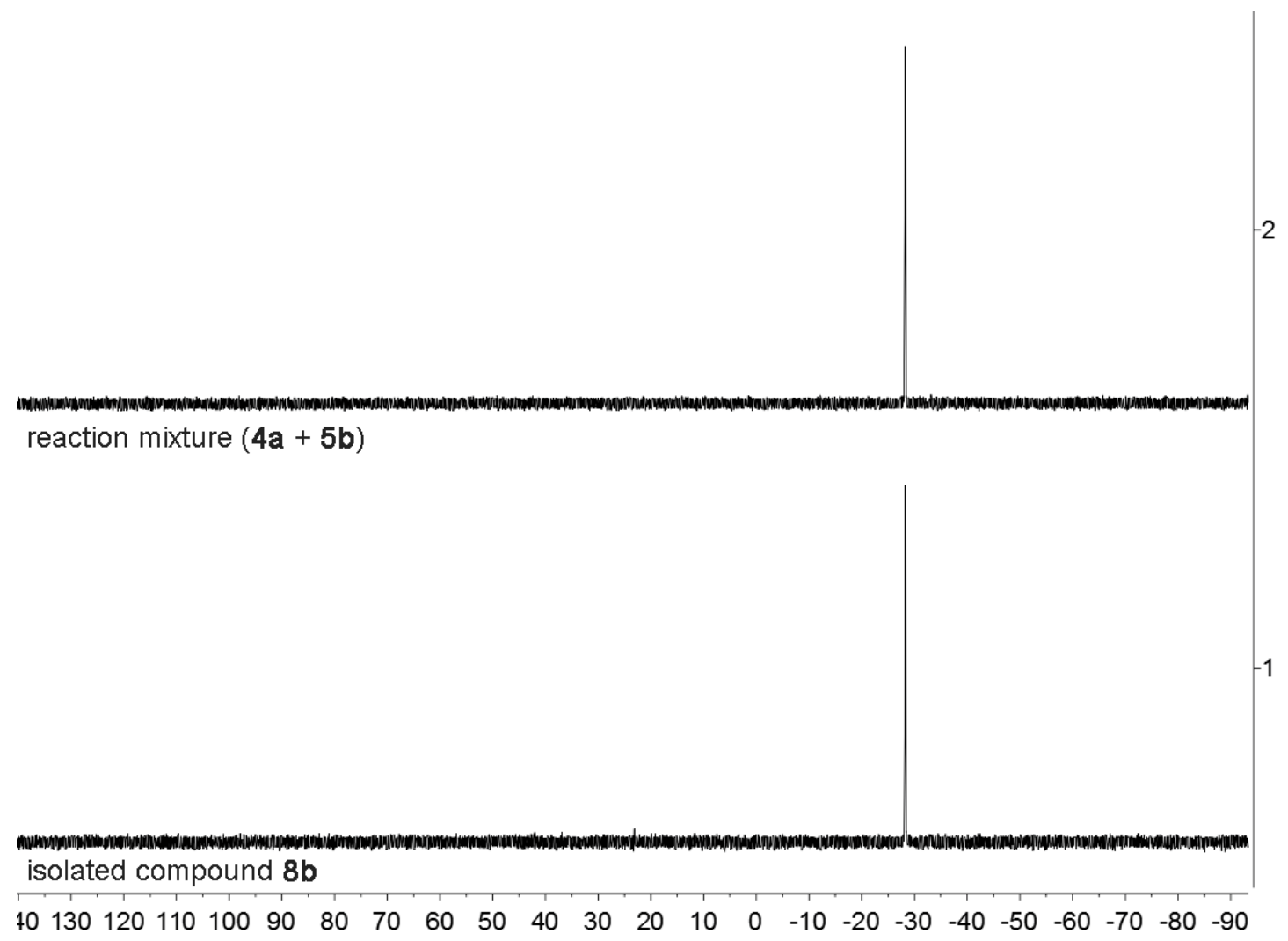

Figure S23. ${ }^{31} \mathbf{P}\left\{{ }^{1} \mathbf{H}\right\}$ NMR $\left(202 \mathrm{MHz}, 299 \mathrm{~K}, \mathrm{C}_{6} \mathrm{D}_{6}\right)$ spectra of (1) isolated compound $\mathbf{8 b}$ and (2) the reaction mixture of the reaction of compounds $\mathbf{4 a}$ with $\mathbf{5 b}$

Comment 2: The reaction of compound $\mathbf{4 a}$ with alkyne $\mathbf{5} \mathbf{c}$ was not completed after 60 hours at r.t.:

a) directly after mixing: reaction mixture of compounds $\mathbf{4 a}$ (ca. $53 \mathrm{~mol} \%$, $\left.{ }^{1} \mathrm{H}\right)$ and $\mathbf{5 c}$, (ca. $\left.47 \mathrm{~mol} \%,{ }^{1} \mathrm{H}\right)$ was observed.

b) after 60 hours r.t.: reaction mixture of compounds $4 \mathbf{a}\left(32 \mathrm{~mol} \%,{ }^{1} \mathrm{H}\right)$, 5c $\left(\right.$ ca. $\left.40 \mathrm{~mol} \%,{ }^{1} \mathrm{H}\right)$, and $8 c\left(28 \mathrm{~mol} \%,{ }^{1} \mathrm{H}\right)$ was observed. 


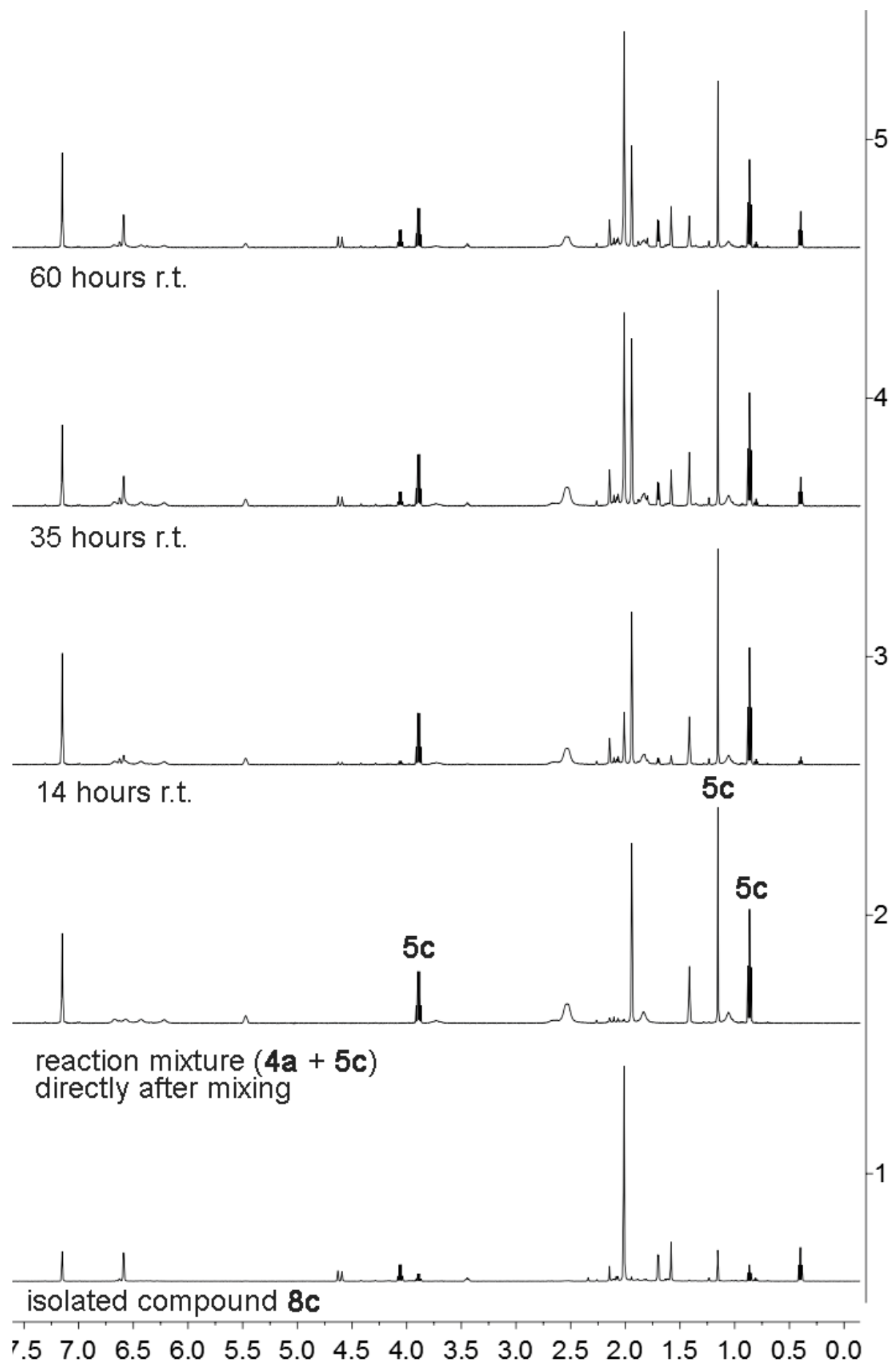

Figure S24. Figure S51. ${ }^{1} \mathrm{H}$ NMR $\left(500 \mathrm{MHz}, 299 \mathrm{~K}, \mathrm{C}_{6} \mathrm{D}_{6}\right)$ spectra of (1) isolated compound $8 \mathbf{c}$ and (2 to 5) the reaction mixture of the reaction of compounds $4 \mathbf{a}$ with $\mathbf{5 c}$ 


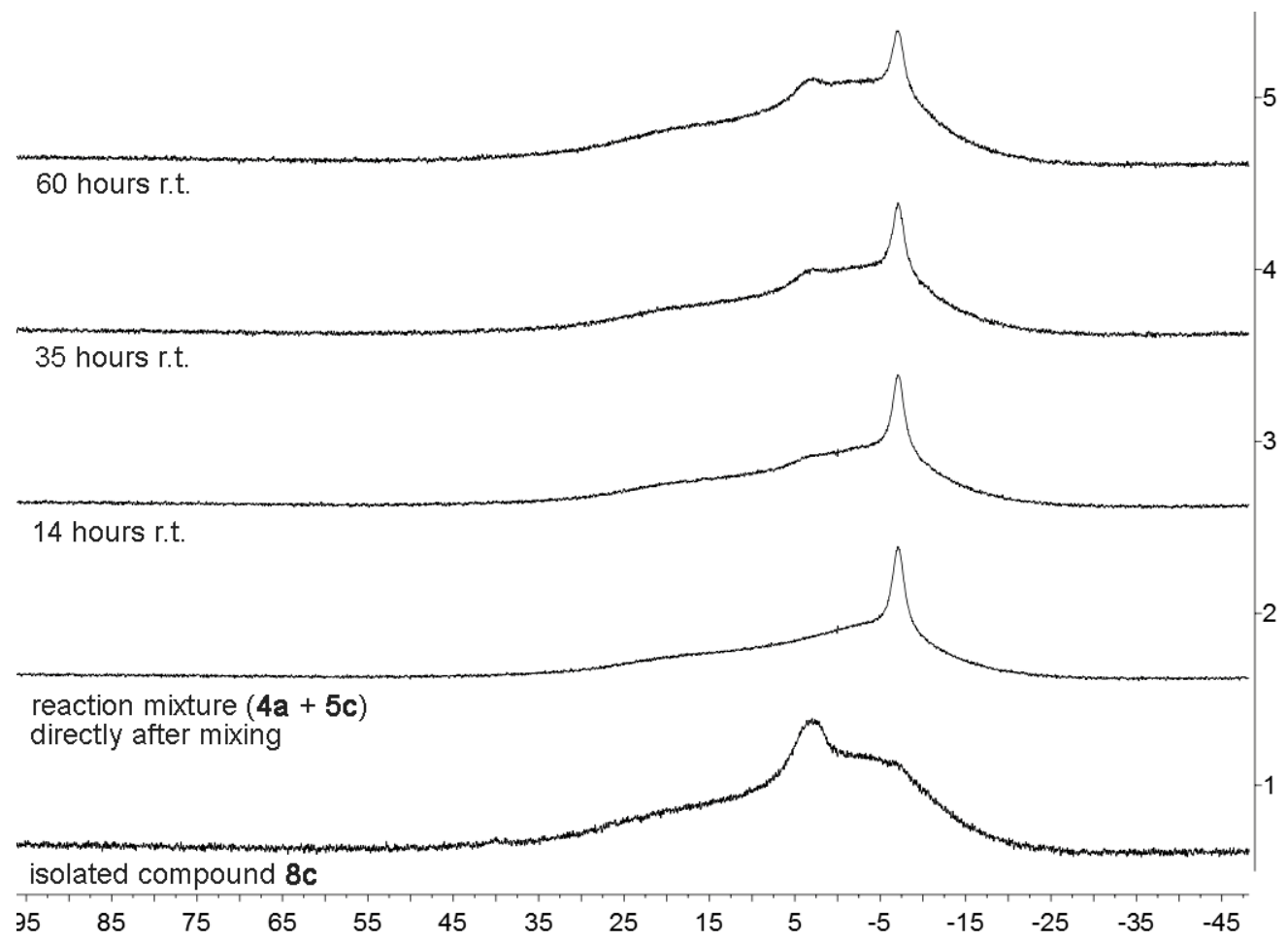

Figure S25. Figure S52. ${ }^{11} \mathbf{B}\left\{{ }^{1} \mathbf{H}\right\}$ NMR $\left(160 \mathrm{MHz}, 299 \mathrm{~K}, \mathrm{C}_{6} \mathrm{D}_{6}\right)$ spectra of (1) isolated compound $8 \mathbf{c}$ and ( 2 to 5 ) the reaction mixture of the reaction of compounds $4 \mathbf{a}$ with $\mathbf{5 c}$

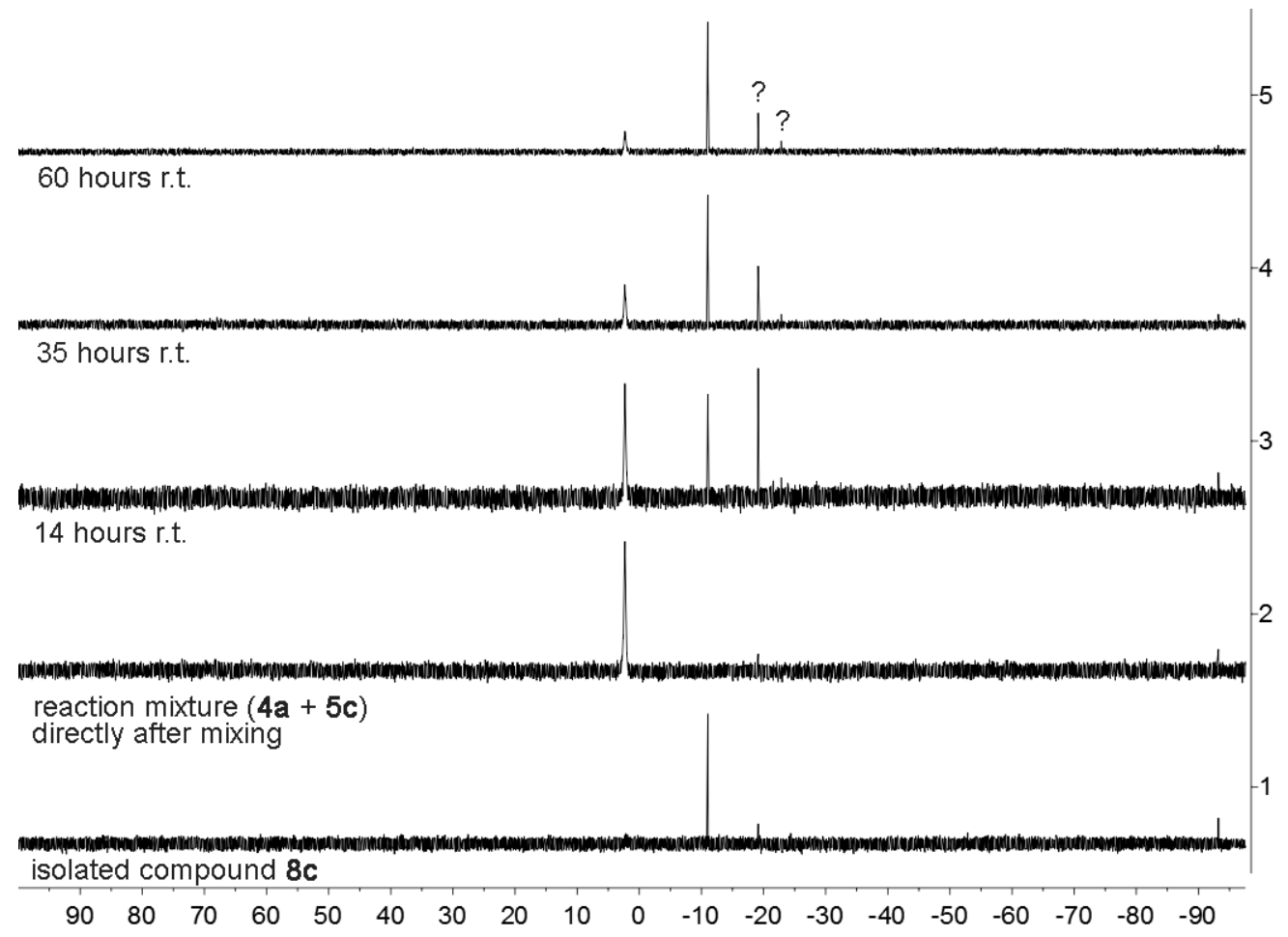

Figure S26. (1) ${ }^{31} \mathbf{P}\left\{{ }^{1} \mathbf{H}\right\}$ NMR (243 MHz, $\left.299 \mathrm{~K}, \mathrm{C}_{6} \mathrm{D}_{6}\right)$ spectrum of isolated compound $\mathbf{8 c}$ and (2 to 5) ${ }^{31} \mathbf{P}\left\{{ }^{1} \mathbf{H}\right\}$ NMR (202 MHz, $\left.299 \mathrm{~K}, \mathrm{C}_{6} \mathrm{D}_{6}\right)$ spectra of the reaction mixture of the reaction of compounds $4 \mathbf{a}$ with $\mathbf{5 c}$ [after 60 hours: $4 \mathbf{a}$ (ca. $48 \mathrm{~mol} \%$ ), $8 \mathbf{c}$ (ca. 41 mol\%), ? (ca. 7 and 4 mol\%)] 


\section{Synthesis of compound 10a}

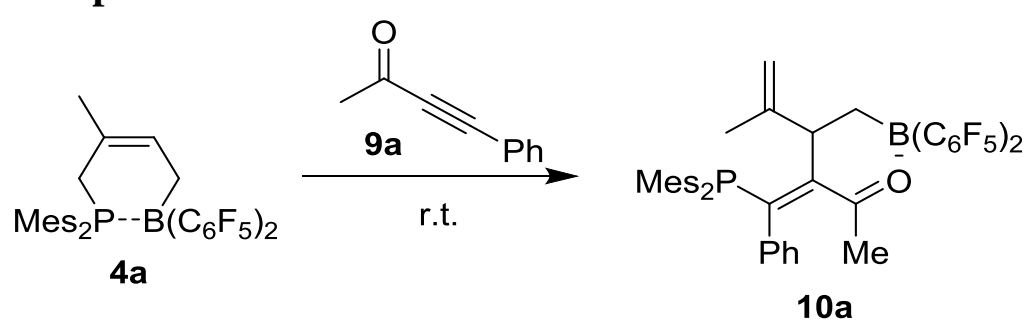

\section{Scheme S4}

A solution of compounds $4 \mathbf{a}(102.3 \mathrm{mg}, 0.15 \mathrm{mmol})$ and $9 \mathrm{a}(21.6 \mathrm{mg}, 0.15 \mathrm{mmol})$ in dichloromethane $(3 \mathrm{~mL})$ was stirred at room temperature for 1 day. Then all volatiles were removed in vacuo and compound 10a $(111.8 \mathrm{mg}, 90 \%)$ was obtained as a dark red solid. Decomp. $168{ }^{\circ} \mathrm{C}$. Anal. Calc. for $\mathrm{C}_{45} \mathrm{H}_{38} \mathrm{BF}_{10} \mathrm{OP}: \mathrm{C}$ : 65.39; H: 4.63. Found: C: $65.23 ; \mathrm{H}: 4.67$.

${ }^{1} \mathbf{H}$ NMR $\left(600 \mathrm{MHz}, 299 \mathrm{~K}, \mathrm{C}_{6} \mathrm{D}_{6}\right): \delta=7.56(\mathrm{~m}, 1 \mathrm{H}, o-\mathrm{Ph}), 6.91\left(\mathrm{~m}, 1 \mathrm{H}, o^{\prime}-\mathrm{Ph}\right), 6.77$ $(\mathrm{m}, 1 \mathrm{H}, p-\mathrm{Ph}), 6.74(\mathrm{~m}, 1 \mathrm{H}, m-\mathrm{Ph}), 6.68\left(\mathrm{~m}, 1 \mathrm{H}, m^{\prime}-\mathrm{Ph}\right), 6.47\left(\mathrm{~d},{ }^{4} J_{\mathrm{PH}}=3.6 \mathrm{~Hz}, 4 \mathrm{H}, m-\right.$ Mes), $4.33\left(\mathrm{~s}, 1 \mathrm{H},=\mathrm{CH}_{2}\right), 4.16\left(\mathrm{~s}, 1 \mathrm{H},=\mathrm{CH}_{2}\right), 3.67(\mathrm{~m}, 1 \mathrm{H}, \mathrm{CH}), 2.17(\mathrm{~s}, 12 \mathrm{H}, o-$ $\left.\mathrm{CH}_{3}{ }^{\mathrm{Mes}}\right), 2.08\left(\mathrm{~m}, 2 \mathrm{H}, \mathrm{CH}_{2}\right), 1.89\left(\mathrm{~s}, 6 \mathrm{H}, p-\mathrm{CH}_{3}{ }^{\mathrm{Mes}}\right), 1.63\left(\mathrm{~d},{ }^{5} \mathrm{~J}_{\mathrm{PH}}=5.0 \mathrm{~Hz}, 3 \mathrm{H},{ }^{\mathrm{O}=\mathrm{C}} \mathrm{CH}_{3}\right)$, $0.99\left(\mathrm{~s}, 3 \mathrm{H}, \mathrm{CH}_{3}\right)$.

${ }^{13} \mathbf{C}\left\{{ }^{1} \mathbf{H}\right\}$ NMR $\left(151 \mathrm{MHz}, 299 \mathrm{~K}, \mathrm{C}_{6} \mathrm{D}_{6}\right): \delta=206.0(\mathrm{br}, \mathrm{C}=\mathrm{O}), 173.2\left(\mathrm{~d},{ }^{1} \mathrm{~J}_{\mathrm{PC}}=7.8 \mathrm{~Hz}\right.$, $=\mathrm{CP}), 146.2\left(\mathrm{~d},{ }^{4} J_{\mathrm{PC}}=1.7 \mathrm{~Hz}, \mathrm{C}={ }^{\mathrm{CH}} 2\right), 143.9\left(\mathrm{~d},{ }^{2} J_{\mathrm{PC}}=15.1 \mathrm{~Hz}, o-\mathrm{Mes}\right), 141.1\left(\mathrm{~d},{ }^{4} J_{\mathrm{PC}}\right.$ $=2.0 \mathrm{~Hz}, p$-Mes), $140.6\left(\mathrm{~d},{ }^{2} J_{\mathrm{PC}}=19.8 \mathrm{~Hz}, i-\mathrm{Ph}\right), 139.6\left(\mathrm{br} \mathrm{d},{ }^{2} J_{\mathrm{PC}}=18.6 \mathrm{~Hz},=\mathrm{C}\right)$, $131.9\left(\mathrm{~d},{ }^{5} J_{\mathrm{PC}}=2.1 \mathrm{~Hz}, p-\mathrm{Ph}\right), 131.5\left(\mathrm{~d},{ }^{3} J_{\mathrm{PC}}=5.2 \mathrm{~Hz}, o^{\prime}-\mathrm{Ph}\right), 130.21\left(\mathrm{~d},{ }^{3} J_{\mathrm{PC}}=19.2\right.$ $\mathrm{Hz}, o-\mathrm{Ph}), 130.17$ (d, $\left.{ }^{3} J_{\mathrm{PC}}=7.6 \mathrm{~Hz}, m-\mathrm{Mes}\right), 128.8(m-\mathrm{Ph}), 128.6$ (m'-Ph), 126.0 (d, ${ }^{1} J_{\mathrm{PC}}=14.0 \mathrm{~Hz}, i$-Mes $), 109.6\left(=\mathrm{CH}_{2}\right), 49.9\left(\mathrm{~d},{ }^{3} J_{\mathrm{PC}}=8.0 \mathrm{~Hz}, \mathrm{CH}\right), 28.9\left(\mathrm{~d},{ }^{4} J_{\mathrm{PC}}=2.1\right.$ $\left.\mathrm{Hz},{ }^{\mathrm{O}=\mathrm{C}} \mathrm{CH}_{3}\right), 23.3\left(\mathrm{~d},{ }^{3} J_{\mathrm{PC}}=13.7 \mathrm{~Hz}, o-\mathrm{CH}_{3}{ }^{\mathrm{Mes}}\right), 23.0\left(\mathrm{br}, \mathrm{CH}_{2}\right), 20.8(\mathrm{~d}, J=0.7 \mathrm{~Hz}, p-$ $\left.\mathrm{CH}_{3}{ }^{\mathrm{Mes}}\right), 20.6\left(\mathrm{CH}_{3}\right),\left[\mathrm{C}_{6} \mathrm{~F}_{5}\right.$ not listed].

${ }^{11} \mathbf{B}\left\{{ }^{1} \mathbf{H}\right\}$ NMR $\left(192 \mathrm{MHz}, 299 \mathrm{~K}, \mathrm{C}_{6} \mathrm{D}_{6}\right): \delta=2.0\left(v_{1 / 2} \sim 500 \mathrm{~Hz}\right)$.

${ }^{10} \mathbf{B}\left\{{ }^{1} \mathbf{H}\right\}$ NMR $\left(64 \mathrm{MHz}, 299 \mathrm{~K}, \mathrm{C}_{6} \mathrm{D}_{6}\right): \delta=2.0\left(v_{1 / 2} \sim 500 \mathrm{~Hz}\right)$.

${ }^{31} \mathbf{P}\left\{{ }^{1} \mathbf{H}\right\}$ NMR $\left(243 \mathrm{MHz}, 299 \mathrm{~K}, \mathrm{C}_{6} \mathrm{D}_{6}\right): \delta=-4.7\left(v_{1 / 2} \sim 40 \mathrm{~Hz}\right)$

${ }^{19}$ F NMR $\left(564 \mathrm{MHz}, 299 \mathrm{~K}, \mathrm{C}_{6} \mathrm{D}_{6}\right): \delta=-133.5(\mathrm{~m}, 2 \mathrm{~F}, o),-158.9\left(\mathrm{t},{ }^{3} J_{\mathrm{FF}}=20.6 \mathrm{~Hz}, 1 \mathrm{~F}\right.$, $p),-164.2(\mathrm{~m}, 2 \mathrm{~F}, m)\left(\mathrm{C}_{6} \mathrm{~F}_{5}\right)\left[\Delta \delta^{19} \mathrm{~F}_{\mathrm{mp}}=5.3\right],-134.9(\mathrm{~m}, 2 \mathrm{~F}, o),-160.6\left(\mathrm{t},{ }^{3} J_{\mathrm{FF}}=20.6 \mathrm{~Hz}\right.$, $1 \mathrm{~F}, p),-165.4(\mathrm{~m}, 2 \mathrm{~F}, m)\left(\mathrm{C}_{6} \mathrm{~F}_{5}\right)\left[\Delta \delta^{19} \mathrm{~F}_{\mathrm{mp}}=4.8\right]$.

${ }^{1} \mathbf{H},{ }^{1} \mathbf{H}$-GCOSY (600 MHz / $\left.600 \mathrm{MHz}, 299 \mathrm{~K}, \mathrm{C}_{6} \mathrm{D}_{6}\right)\left[\right.$ selected traces]: $\delta{ }^{1} \mathrm{H} / \delta^{1} \mathrm{H}=$ $7.56 / 6.74(o-\mathrm{Ph} / m-\mathrm{Ph}), 6.91 / 6.68\left(o^{\prime}-\mathrm{Ph} / m^{\prime}-\mathrm{Ph}\right), 4.33,4.16 / 3.67,0.99\left(=\mathrm{CH}_{2}\right.$, $\left.=\mathrm{CH}_{2} / \mathrm{CH}, \mathrm{CH}_{3}\right), 3.67 / 2.08\left(\mathrm{CH} / \mathrm{CH}_{2}\right)$.

${ }^{1} \mathbf{H},{ }^{13} \mathbf{C}$-GHSQC $\left(600 \mathrm{MHz} / 151 \mathrm{MHz}, 299 \mathrm{~K}, \mathrm{C}_{6} \mathrm{D}_{6}\right): \delta{ }^{1} \mathrm{H} / \delta{ }^{13} \mathrm{C}=7.56 / 130.21(o-$ $\mathrm{Ph}), 6.91 / 131.5\left(o^{\prime}-\mathrm{Ph}\right), 6.77 / 131.9(p-\mathrm{Ph}), 6.74 / 128.8(m-\mathrm{Ph}), 6.68 / 128.6\left(m^{\prime}-\mathrm{Ph}\right)$, 6.47 / 130.17 (m-Mes), 4.33, 4.16 / $109.6\left(=\mathrm{CH}_{2}\right), 3.67$ / $49.9(\mathrm{CH}), 2.17 / 23.3$ (o$\left.\mathrm{CH}_{3}{ }^{\mathrm{Mes}}\right), 2.08 / 23.0\left(\mathrm{CH}_{2}\right), 1.89 / 20.8\left(p-\mathrm{CH}_{3}{ }^{\mathrm{Mes}}\right), 1.63 / 28.9\left({ }^{\mathrm{O}=\mathrm{C}} \mathrm{CH}_{3}\right), 0.99 / 20.6$ 
$\left(\mathrm{CH}_{3}\right)$.

${ }^{1} \mathbf{H},{ }^{13} \mathbf{C}-\mathbf{G H M B C}\left(600 \mathrm{MHz} / 151 \mathrm{MHz}, 299 \mathrm{~K}, \mathrm{C}_{6} \mathrm{D}_{6}\right)$ [selected traces]: $\delta{ }^{1} \mathrm{H} / \delta^{13} \mathrm{C}=$ 7.56, 6.91, 3.67 / $173.2(o-\mathrm{Ph}, o '-\mathrm{Ph}, \mathrm{CH} /=\mathrm{CP}), 6.74,6.68 / 140.6\left(m-\mathrm{Ph}, m^{\prime}-\mathrm{Ph} / i-\right.$ $\mathrm{Ph}), 6.47,2.17 / 126.0$ ( $m$-Mes, $o-\mathrm{CH}_{3}{ }^{\mathrm{Mes}} / i$-Mes $\left.{ }^{\mathrm{a}}\right), 4.33,4.16,3.67,2.08,0.99 / 146.2$ $\left(=\mathrm{CH}_{2},=\mathrm{CH}_{2}, \mathrm{CH}, \mathrm{CH}_{2}, \mathrm{CH}_{3} / \mathrm{C}={ }^{\mathrm{CH}} 2\right), 3.67,2.08,1.63 / 139.6\left(\mathrm{CH}, \mathrm{CH}_{2},{ }^{\mathrm{O}=\mathrm{C}} \mathrm{CH}_{3} /=\mathrm{C}\right)$, 3.67, $1.63 / 206.0\left(\mathrm{CH},{ }^{\mathrm{O}=\mathrm{C}} \mathrm{CH}_{3} / \mathrm{C}=\mathrm{O}\right), 2.17 / 143.9$ (o- $\left.\mathrm{CH}_{3}{ }^{\text {Mes }} / o-\mathrm{Mes}\right), 1.89 / 141.1$ $\left(p-\mathrm{CH}_{3}{ }^{\mathrm{Mes}} / p\right.$-Mes).

${ }^{19} \mathbf{F},{ }^{19} \mathbf{F}-G C O S Y\left(564 \mathrm{MHz} / 564 \mathrm{MHz}, 299 \mathrm{~K}, \mathrm{C}_{6} \mathrm{D}_{6}\right): \delta{ }^{19} \mathrm{~F} / \delta^{19} \mathrm{~F}=-164.2 /-133.5,-$ $158.9\left(m-\mathrm{C}_{6} \mathrm{~F}_{5}^{\mathrm{a}} / o-\mathrm{C}_{6} \mathrm{~F}_{5}{ }^{\mathrm{a}}, p-\mathrm{C}_{6} \mathrm{~F}_{5}^{\mathrm{a}}\right),-165.4 /-134.9,-160.6\left(m-\mathrm{C}_{6} \mathrm{~F}_{5} \mathrm{~b} / o-\mathrm{C}_{6} \mathrm{~F}_{5}^{\mathrm{b}}, p-\mathrm{C}_{6} \mathrm{~F}_{5}{ }^{\mathrm{b}}\right)$.

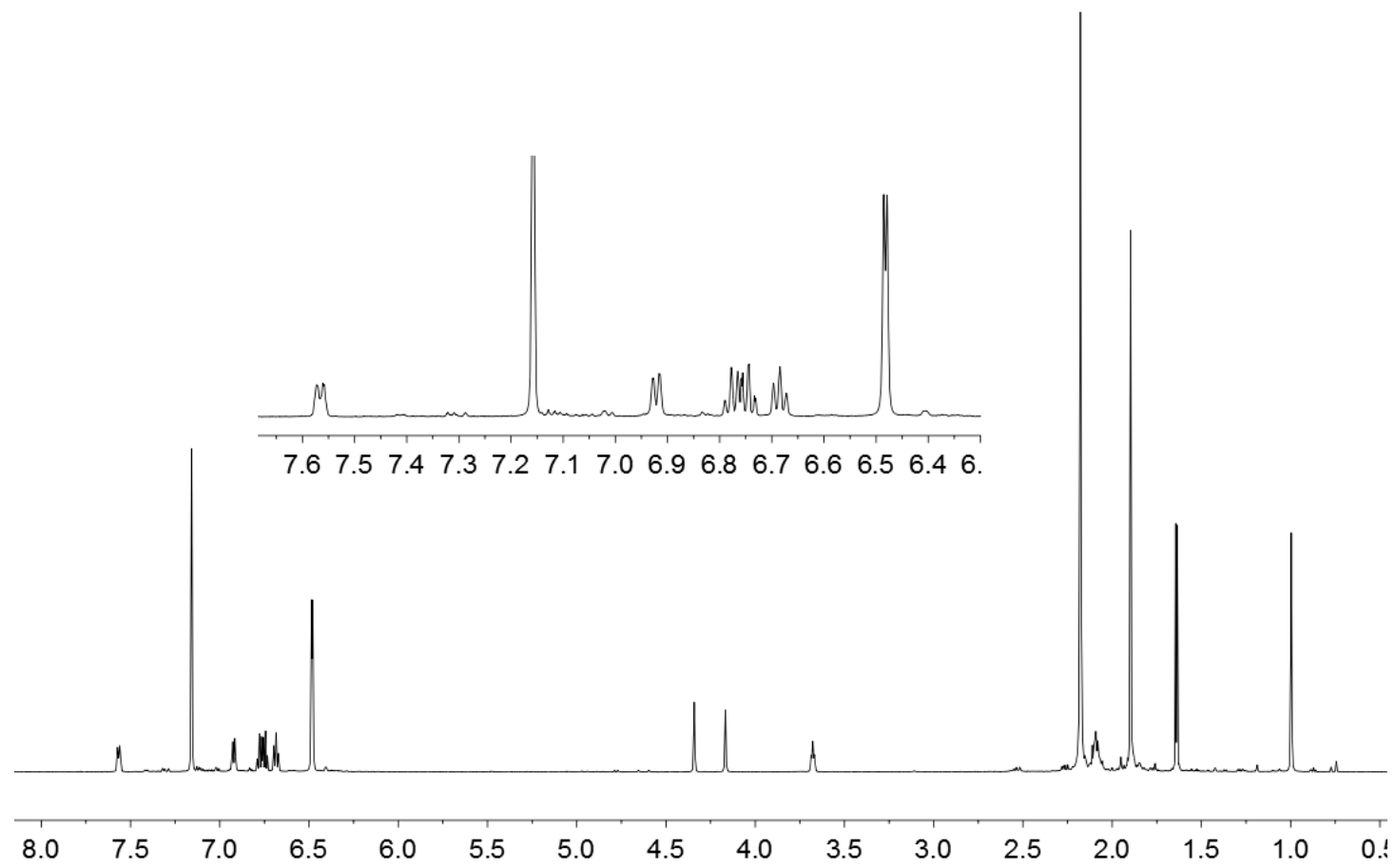

Figure S27. ${ }^{1} \mathbf{H}$ NMR $\left(600 \mathrm{MHz}, 299 \mathrm{~K}, \mathrm{C}_{6} \mathrm{D}_{6}\right)$ of compound 10a 


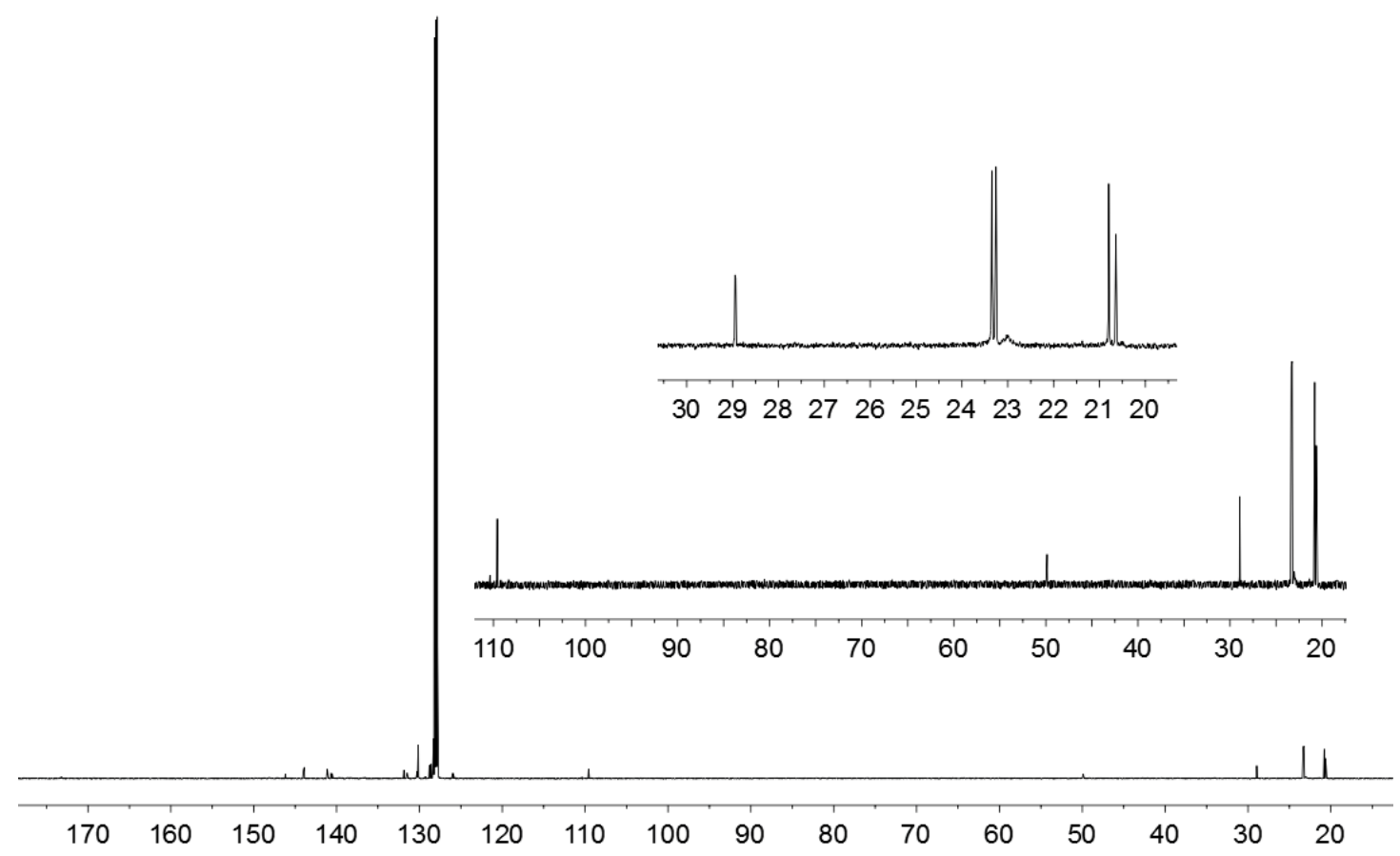

Figure S28. ${ }^{13} \mathbf{C}\left\{{ }^{1} \mathbf{H}\right\}$ NMR $\left(151 \mathrm{MHz}, 299 \mathrm{~K}, \mathrm{C}_{6} \mathrm{D}_{6}\right)$ of compound 10a
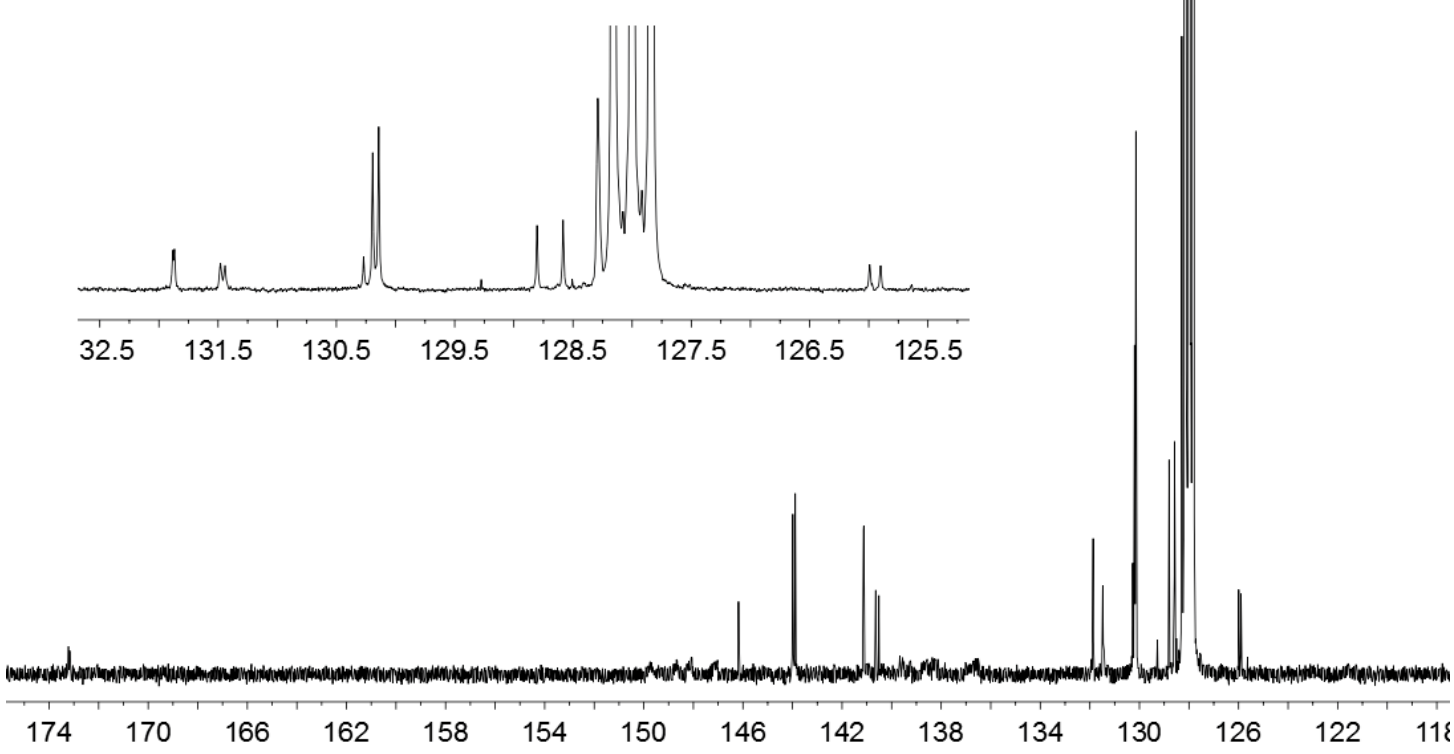

Figure S29. ${ }^{13} \mathbf{C}\left\{{ }^{1} \mathbf{H}\right\}$ NMR $\left(151 \mathrm{MHz}, 299 \mathrm{~K}, \mathrm{C}_{6} \mathrm{D}_{6}\right)$ of compound 10a 


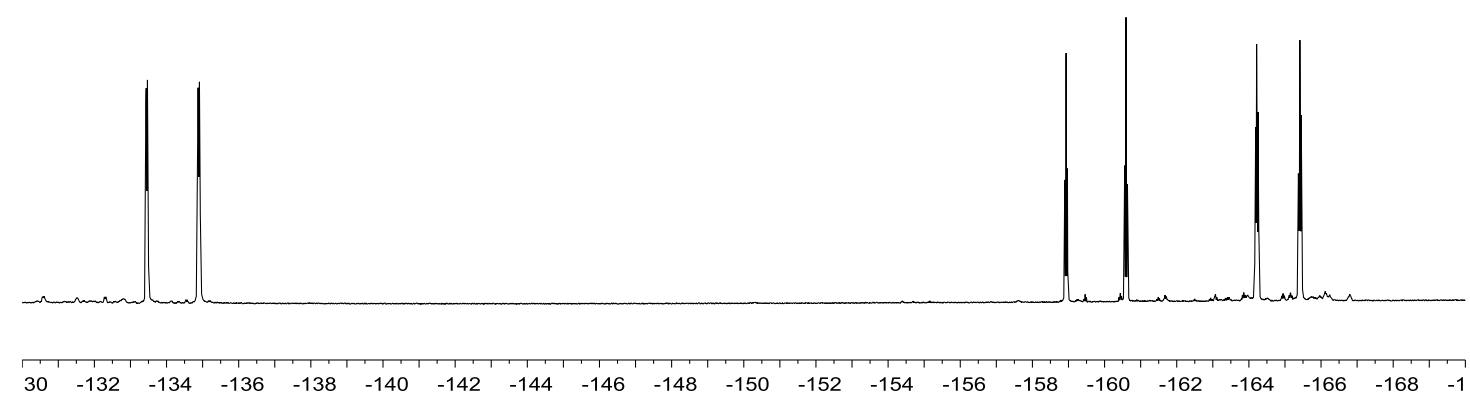

Figure S30. ${ }^{19}$ F NMR $\left(564 \mathrm{MHz}, 299 \mathrm{~K}, \mathrm{C}_{6} \mathrm{D}_{6}\right)$ of compound 10a

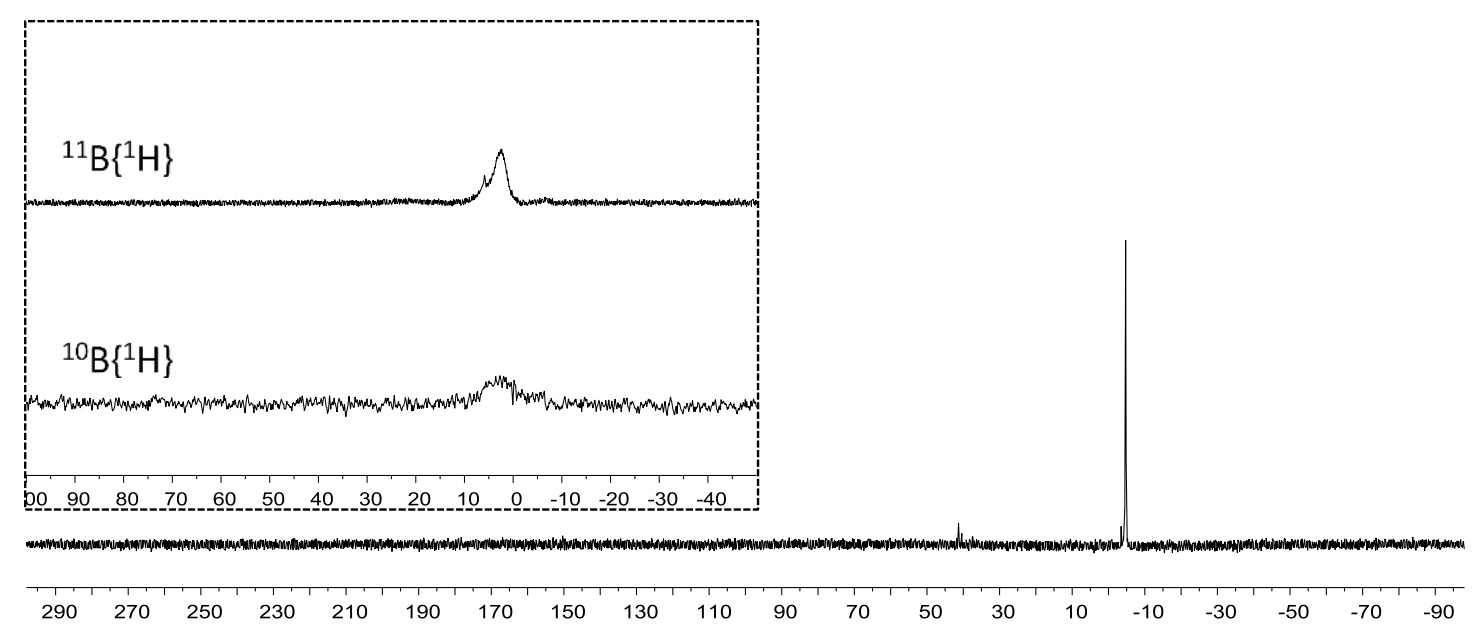

Figure S31. ${ }^{11} \mathbf{B}\left\{{ }^{1} \mathbf{H}\right\}$ NMR $\left(192 \mathrm{MHz}, 299 \mathrm{~K}, \mathrm{C}_{6} \mathrm{D}_{6}\right),{ }^{10} \mathbf{B}\left\{{ }^{1} \mathbf{H}\right\}$ NMR $(64 \mathrm{MHz}, 299 \mathrm{~K}$, $\left.\mathrm{C}_{6} \mathrm{D}_{6}\right)$ and ${ }^{31} \mathbf{P}\left\{{ }^{1} \mathbf{H}\right\} \mathbf{N M R}\left(243 \mathrm{MHz}, 299 \mathrm{~K}, \mathrm{C}_{6} \mathrm{D}_{6}\right)$ of compound $\mathbf{1 0 a}$ 


\section{Synthesis of compound $10 \mathrm{~b}$}

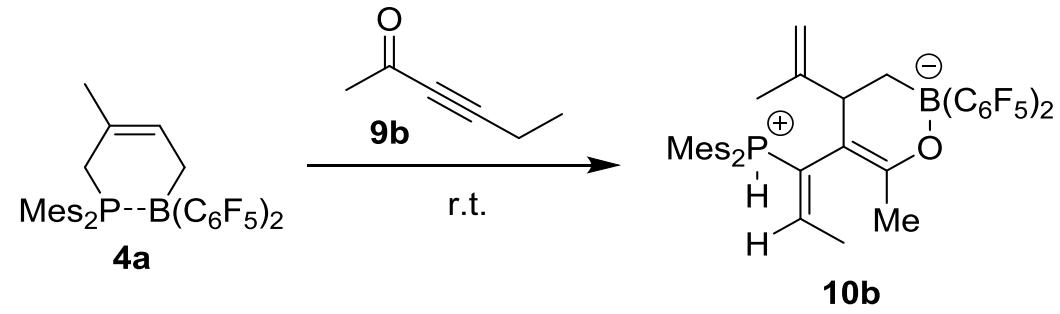

Scheme S5

A solution of compound $4 \mathbf{a}(102.3 \mathrm{mg}, 0.15 \mathrm{mmol})$ and $9 \mathbf{b}(14.4 \mathrm{mg}, 0.15 \mathrm{mmol})$ in pentane $(3 \mathrm{~mL})$ was stirred at room temperature for 5 days. Then all volatiles were removed in vacuo and the residue was washed with cold pentane $(3 \times 1 \mathrm{~mL})$. After drying in vacuo compound $\mathbf{1 0 b}(88.5 \mathrm{mg}, 76 \%)$ was obtained as a yellow solid. Decomp. 169 ${ }^{\circ} \mathrm{C}$. Anal. Calc. for $\mathrm{C}_{41} \mathrm{H}_{38} \mathrm{BF}_{10} \mathrm{O}_{2} \mathrm{P}: \mathrm{C}: 61.98 ; \mathrm{H}: 4.82$. Found: $\mathrm{C}: 62.41 ; \mathrm{H}: 4.68$.

${ }^{1} \mathbf{H}$ NMR $\left(500 \mathrm{MHz}, 299 \mathrm{~K}, \mathrm{CD}_{2} \mathrm{Cl}_{2}\right): \delta=8.13\left(\mathrm{br} \mathrm{d},{ }^{1} J_{\mathrm{PH}}=480.5 \mathrm{~Hz}, 1 \mathrm{H}, \mathrm{PH}\right), 7.05$ $\left(\mathrm{br}, 2 \mathrm{H}, m-\mathrm{Mes}^{\mathrm{a}}\right), 6.93\left(\mathrm{br}, 2 \mathrm{H}, m-\mathrm{Mes}^{\mathrm{b}}\right), 5.87\left(\mathrm{dqd},{ }^{3} J_{\mathrm{PH}}=27.3 \mathrm{~Hz},{ }^{3} J_{\mathrm{HH}}=6.9 \mathrm{~Hz},{ }^{4} J_{\mathrm{HH}}\right.$ $=1.1 \mathrm{~Hz}, 1 \mathrm{H},=\mathrm{CH}), 4.70\left(\mathrm{~s}, 1 \mathrm{H},=\mathrm{CH}_{2}\right), 4.59\left(\mathrm{~s}, 1 \mathrm{H},=\mathrm{CH}_{2}\right), 2.84(\mathrm{~m}, 1 \mathrm{H}, \mathrm{CH}), 2.35$ (s, 3H, $p-\mathrm{CH}_{3}{ }^{\mathrm{Mes}, \mathrm{b}}$ ), 2.34 (s, 3H, $p-\mathrm{CH}_{3}{ }^{\mathrm{Mes}, \mathrm{a}}$ ), 2.24 (br, $12 \mathrm{H}, o-\mathrm{CH}_{3}{ }^{\mathrm{Mes}, \mathrm{a}, \mathrm{b}}$ ), 1.93 (ddd, $\left.{ }^{3} J_{\mathrm{HH}}=6.9 \mathrm{~Hz},{ }^{4} J_{\mathrm{PH}}=3.7 \mathrm{~Hz},{ }^{\mathrm{a}} J_{\mathrm{HH}}=2.0 \mathrm{~Hz},{ }^{\mathrm{a}} 3 \mathrm{H},{ }^{\mathrm{CH}} \mathrm{CH}_{3}\right), 1.78\left(\mathrm{~m}, 3 \mathrm{H},{ }^{\mathrm{O}-\mathrm{C}} \mathrm{CH}_{3}\right), 1.71$ $\left(\mathrm{m}, 3 \mathrm{H}, \mathrm{CH}_{3}\right), 1.32\left(\mathrm{dd},{ }^{2} J_{\mathrm{HH}}=13.2 \mathrm{~Hz},{ }^{3} J_{\mathrm{HH}}=5.9 \mathrm{~Hz}, 1 \mathrm{H}, \mathrm{CH}_{2}\right), 1.16\left(\mathrm{dd},{ }^{2} J_{\mathrm{HH}}=13.2\right.$ $\left.\mathrm{Hz},{ }^{3} \mathrm{~J}_{\mathrm{HH}}=10.0 \mathrm{~Hz}, 1 \mathrm{H}, \mathrm{CH}_{2}\right),\left[{ }^{\mathrm{a}}\right.$ assignment by ${ }^{1} \mathrm{H}\left\{{ }^{1} \mathrm{H}\right\}$ and ${ }^{1} \mathrm{H}\left\{{ }^{31} \mathrm{P}\right\}$ NMR experiments $]$.

${ }^{13} \mathbf{C}\left\{{ }^{1} \mathbf{H}\right\}$ NMR $\left(126 \mathrm{MHz}, 299 \mathrm{~K}, \mathrm{CD}_{2} \mathrm{Cl}_{2}\right): \delta=160.5\left(\mathrm{~d},{ }^{3} \mathrm{JPC}_{\mathrm{PC}}=12.4 \mathrm{~Hz},=\mathrm{CO}\right), 154.1$ $\left(\mathrm{br}, \mathrm{C}={ }^{\mathrm{CH}} 2\right), 146.1\left(\mathrm{~d},{ }^{5} J_{\mathrm{PC}}=2.7 \mathrm{~Hz}, p-\mathrm{Mes}^{\mathrm{a}}\right), 145.8\left(\mathrm{~d},{ }^{5} J_{\mathrm{PC}}=2.7 \mathrm{~Hz}, p-\mathrm{Mes}^{\mathrm{b}}\right), 144.5$ $\left(\mathrm{d},{ }^{2} J_{\mathrm{PC}}=9.5 \mathrm{~Hz}\right), 143.3(\mathrm{br})(o-\mathrm{Mes})^{\mathrm{t}}, 143.3\left(\mathrm{~d},{ }^{2} J_{\mathrm{PC}}=19.9 \mathrm{~Hz},=\mathrm{CH}\right), 131.8(\mathrm{br}, m-$ $\left.\mathrm{Mes}^{\mathrm{a}}\right), 131.5\left(\mathrm{~d},{ }^{3} J_{\mathrm{PC}}=10.8 \mathrm{~Hz}, m-\mathrm{Mes}^{\mathrm{b}}\right), 126.8\left(\mathrm{~d},{ }^{1} J_{\mathrm{PC}}=60.3 \mathrm{~Hz},=\mathrm{CP}\right), 112.8\left(\mathrm{~d},{ }^{1} J_{\mathrm{PC}}\right.$ $=76.8 \mathrm{~Hz}), 112.6\left(\mathrm{~d},{ }^{1} J_{\mathrm{PC}}=79.2 \mathrm{~Hz}, i-\mathrm{Mes}^{\mathrm{a}, \mathrm{b}}\right)^{\mathrm{t}}, 110.7\left(\mathrm{~d},{ }^{5} J_{\mathrm{PC}}=1.5 \mathrm{~Hz},=\mathrm{CH}_{2}\right), 100.6$ $\left(\mathrm{d},{ }^{3} J_{\mathrm{PC}}=9.5 \mathrm{~Hz},=\mathrm{C}\right), 45.3(\mathrm{CH}), 26.1\left(\mathrm{br}, \mathrm{CH}_{2}\right), 23.2\left(\mathrm{~d},{ }^{4} \mathrm{~J}_{\mathrm{PC}}=1.8 \mathrm{~Hz},{ }^{\mathrm{O}-\mathrm{C}} \mathrm{CH}_{3}\right), 22.2$, 22.1 (each br,,$\left.o-\mathrm{CH}_{3}{ }^{\mathrm{Mes}, \mathrm{a}, \mathrm{b}}\right), 21.4\left(\mathrm{~d},{ }^{5} \mathrm{~J}_{\mathrm{PC}}=1.3 \mathrm{~Hz}, p-\mathrm{CH}_{3}{ }^{\mathrm{Mes}, \mathrm{a}}\right), 21.2\left(p-\mathrm{CH}_{3}{ }^{\mathrm{Mes}, \mathrm{b}}\right), 19.8$ $\left(\mathrm{d},{ }^{3} \mathrm{~J}_{\mathrm{PC}}=18.5 \mathrm{~Hz},{ }^{\mathrm{CH}} \mathrm{CH}_{3}\right), 19.6\left(\mathrm{br}, \mathrm{CH}_{3}\right)$, [ ${ }^{\mathrm{t}}$ tentatively assigned; $\mathrm{C}_{6} \mathrm{~F}_{5}$ not listed $]$.

${ }^{11} \mathbf{B}\left\{{ }^{1} \mathrm{H}\right\}$ NMR $\left(160 \mathrm{MHz}, 299 \mathrm{~K}, \mathrm{CD}_{2} \mathrm{Cl}_{2}\right): \delta=-2.4\left(v_{1 / 2} \sim 160 \mathrm{~Hz}\right)$.

${ }^{31} \mathbf{P}\left\{{ }^{1} \mathbf{H}\right\}$ NMR $\left(202 \mathrm{MHz}, 299 \mathrm{~K}, \mathrm{CD}_{2} \mathrm{Cl}_{2}\right): \delta=-19.8\left(v_{1 / 2} \sim 10 \mathrm{~Hz}\right)$.

${ }^{31} \mathbf{P}$ NMR $\left(202 \mathrm{MHz}, 299 \mathrm{~K}, \mathrm{CD}_{2} \mathrm{Cl}_{2}\right): \delta=-19.8\left(\mathrm{br} \mathrm{dd},{ }^{1} J_{\mathrm{PH}} \sim 480 \mathrm{~Hz},{ }^{3} J_{\mathrm{PH}} \sim 26 \mathrm{~Hz}\right)$.

${ }^{19}$ F NMR $\left(470 \mathrm{MHz}, 299 \mathrm{~K}, \mathrm{CD}_{2} \mathrm{Cl}_{2}\right): \delta=-134.8(\mathrm{~m}, 2 \mathrm{~F}, o),-163.2\left(\mathrm{t},{ }^{3} J_{\mathrm{FF}}=20.3 \mathrm{~Hz}\right.$, $1 \mathrm{~F}, p),-166.5(\mathrm{~m}, 2 \mathrm{~F}, m)\left(\mathrm{C}_{6} \mathrm{~F}_{5}\right)\left[\Delta \delta^{19} \mathrm{~F}_{\mathrm{mp}}=3.3\right],-135.6(\mathrm{br} \mathrm{m}, 2 \mathrm{~F}, o),-164.0\left(\mathrm{br} \mathrm{t},{ }^{3} J_{\mathrm{FF}}=\right.$ $20.1 \mathrm{~Hz}, 1 \mathrm{~F}, p),-167.3$ (br m, $2 \mathrm{~F}, m)\left(\mathrm{C}_{6} \mathrm{~F}_{5}\right)\left[\Delta \delta^{19} \mathrm{~F}_{\mathrm{mp}}=3.3\right]$.

${ }^{1} \mathbf{H},{ }^{1} \mathbf{H}-\mathbf{G C O S Y}\left(500 \mathrm{MHz} / 500 \mathrm{MHz}, 299 \mathrm{~K}, \mathrm{CD}_{2} \mathrm{Cl}_{2}\right.$ )[selected traces]: $\delta{ }^{1} \mathrm{H} / \delta^{1} \mathrm{H}=$ $7.05 / 2.34\left(m-\mathrm{Mes}^{\mathrm{a}} / p-\mathrm{CH}_{3}{ }^{\mathrm{Mes}, \mathrm{a}}\right), 6.93 / 2.35\left(m-\mathrm{Mes}^{\mathrm{b}} / p-\mathrm{CH}_{3}{ }^{\mathrm{Mes}, \mathrm{b}}\right), 5.87 / 1.93$ (=CH $\left./={ }^{\mathrm{CH}} \mathrm{CH}_{3}\right), 4.70,4.59 / 1.71\left(=\mathrm{CH}_{2},=\mathrm{CH}_{2} / \mathrm{CH}_{3}\right), 2.84 / 1.32,1.16\left(\mathrm{CH} / \mathrm{CH}_{2}, \mathrm{CH}_{2}\right)$.

${ }^{1} \mathbf{H},{ }^{13} \mathbf{C}$-GHSQC (500 MHz / $\left.126 \mathrm{MHz}, 299 \mathrm{~K}, \mathrm{CD}_{2} \mathrm{Cl}_{2}\right): \delta{ }^{1} \mathrm{H} / \delta{ }^{13} \mathrm{C}=7.05 / 131.8$ 
$\left(m-\mathrm{Mes}^{\mathrm{a}}\right), 6.93 / 131.5\left(m-\mathrm{Mes}^{\mathrm{b}}\right), 5.87 / 143.3(=\mathrm{CH}), 4.70,4.59 / 110.7\left(=\mathrm{CH}_{2}\right), 2.84 /$ $45.3(\mathrm{CH}), 2.35 / 21.2\left(p-\mathrm{CH}_{3}{ }^{\text {Mes,b }}\right), 2.34 / 21.4\left(p-\mathrm{CH}_{3}{ }^{\mathrm{Mes}, \mathrm{a}}\right), 2.24 / 22.2,22.1$ (o$\left.\mathrm{CH}_{3}{ }^{\mathrm{Mes}, \mathrm{a}, \mathrm{b}}\right), 1.93 / 19.8\left({ }^{=\mathrm{CH}} \mathrm{CH}_{3}\right), 1.78 / 23.2\left({ }^{\mathrm{O}-\mathrm{C}} \mathrm{CH}_{3}\right), 1.71 / 19.6\left(\mathrm{CH}_{3}\right), 1.32,1.16$ / $26.1\left(\mathrm{CH}_{2}\right)$.

${ }^{1} \mathbf{H},{ }^{13} \mathbf{C}-\mathbf{G H M B C}\left(500 \mathrm{MHz} / 126 \mathrm{MHz}, 299 \mathrm{~K}, \mathrm{CD}_{2} \mathrm{Cl}_{2}\right)$ [selected traces]: $\delta{ }^{1} \mathrm{H} / \delta{ }^{13} \mathrm{C}$ $=5.87,1.78,1.32 / 100.6\left(=\mathrm{CH},{ }^{\mathrm{O}-\mathrm{C}} \mathrm{CH}_{3}, \mathrm{CH}_{2} /=\mathrm{C}\right), 2.35 / 145.8\left(p-\mathrm{CH}_{3}{ }^{\mathrm{Mes}, \mathrm{b}} / p-\mathrm{Mes}^{\mathrm{b}}\right)$, $2.34 / 146.1\left(p-\mathrm{CH}_{3}{ }^{\mathrm{Mes}, \mathrm{a}} / p-\mathrm{Mes}^{\mathrm{a}}\right), 1.93 / 126.8\left({ }^{-\mathrm{CH}} \mathrm{CH}_{3} /=\mathrm{CP}\right), 1.78 / 160.5\left({ }^{\mathrm{O}-\mathrm{C}} \mathrm{CH}_{3} /\right.$ $=\mathrm{CO}), 1.71 / 154.1\left(\mathrm{CH}_{3} / \mathrm{C}={ }^{\mathrm{CH}} 2\right)$.

${ }^{19} \mathbf{F},{ }^{19} \mathbf{F}-G C O S Y\left(470 \mathrm{MHz} / 470 \mathrm{MHz}, 299 \mathrm{~K}, \mathrm{CD}_{2} \mathrm{Cl}_{2}\right): \delta{ }^{19} \mathrm{~F} / \delta{ }^{19} \mathrm{~F}=-166.5 /-134.8$, $-163.2\left(m-\mathrm{C}_{6} \mathrm{~F}_{5}^{\mathrm{a}} / o-\mathrm{C}_{6} \mathrm{~F}_{5}^{\mathrm{a}}, p-\mathrm{C}_{6} \mathrm{~F}_{5}^{\mathrm{a}}\right),-167.3 /-135.6,-164.0\left(m-\mathrm{C}_{6} \mathrm{~F}_{5}{ }^{\mathrm{b}} / o-\mathrm{C}_{6} \mathrm{~F}_{5}{ }^{\mathrm{b}}, p-\mathrm{C}_{6} \mathrm{~F}_{5}^{\mathrm{b}}\right)$.

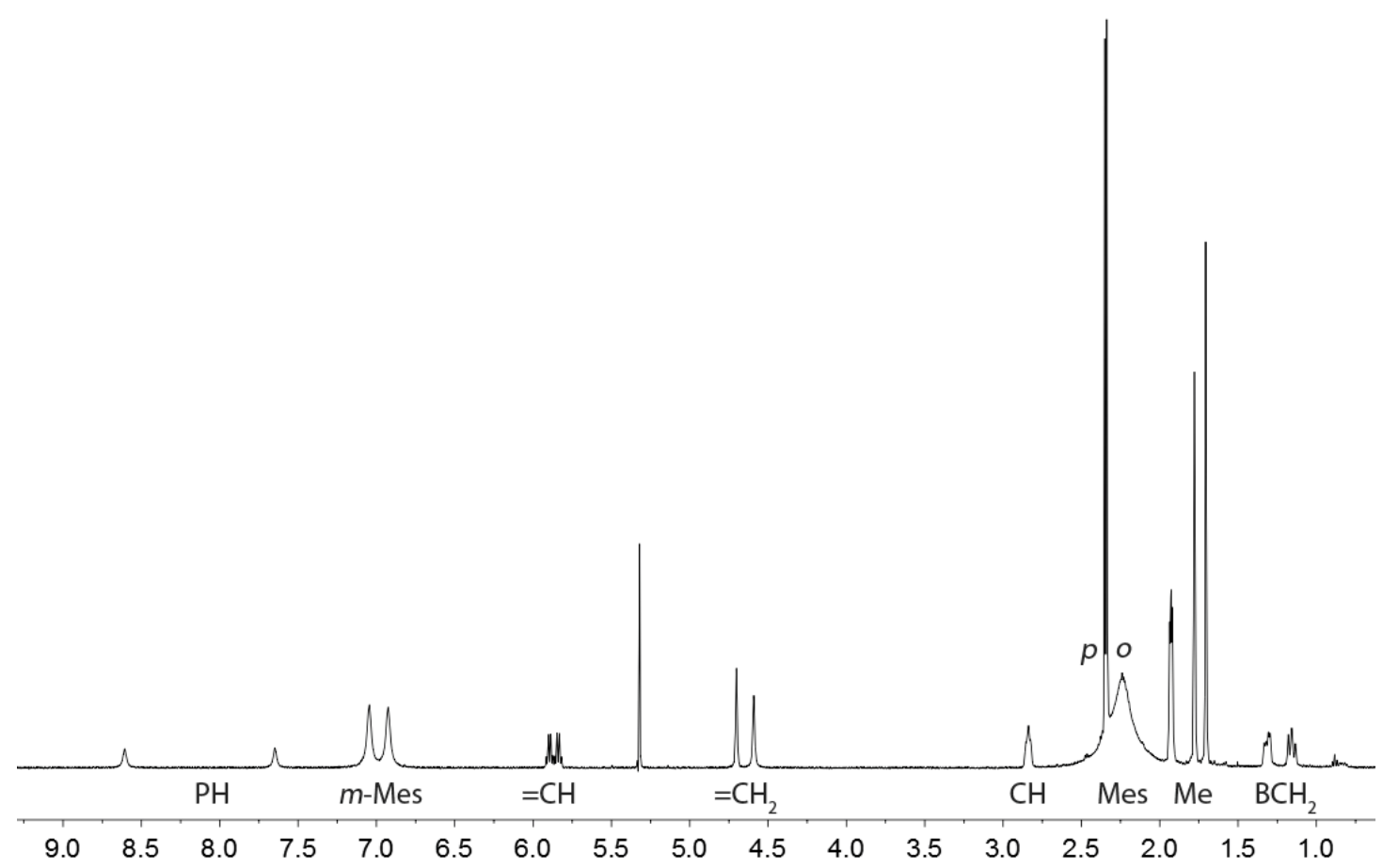

Figure S32. ${ }^{1} \mathbf{H}$ NMR $\left(500 \mathrm{MHz}, 299 \mathrm{~K}, \mathrm{CD}_{2} \mathrm{Cl}_{2}\right)$ of compound $\mathbf{1 0 b}$ 

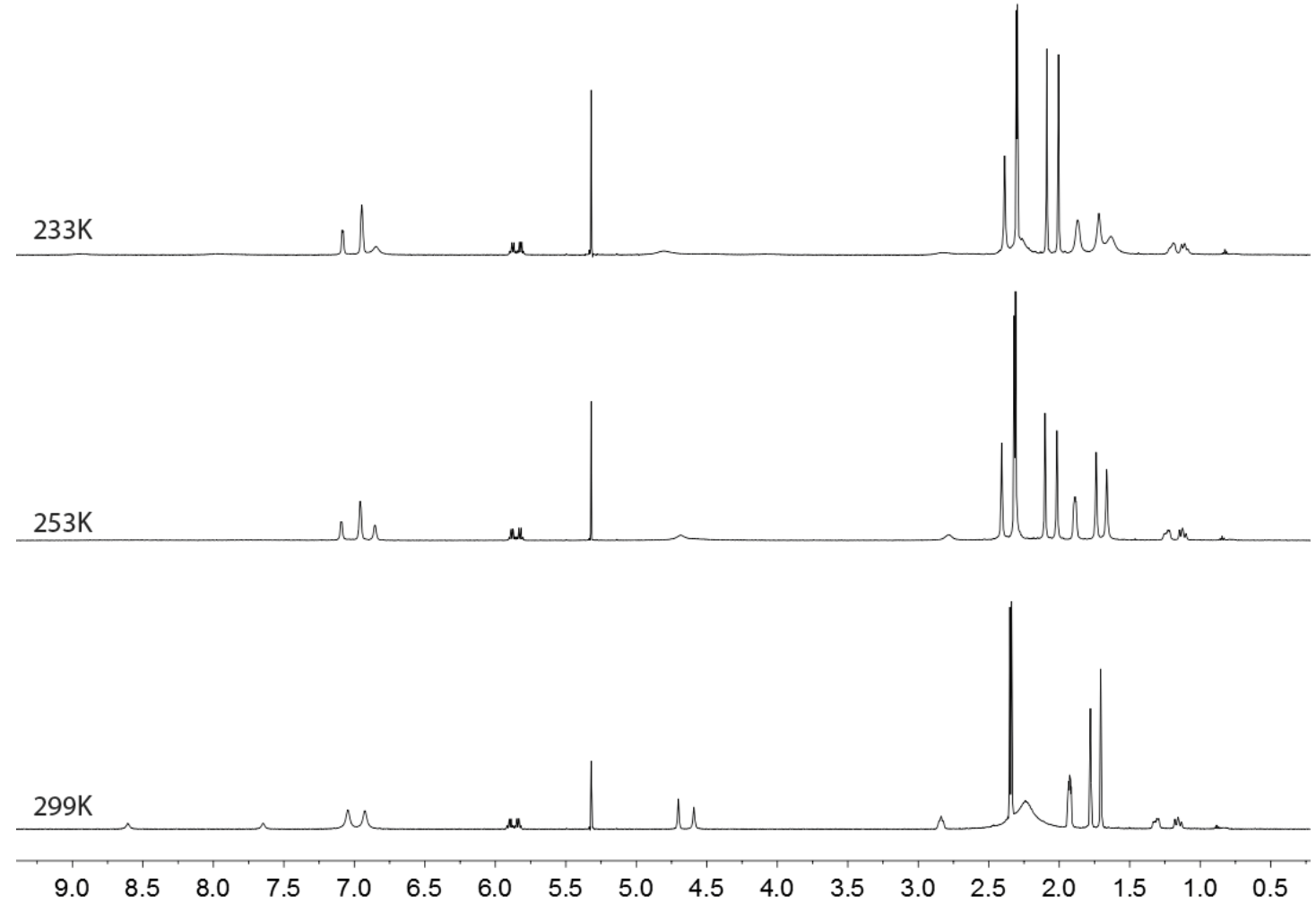

Figure S33. ${ }^{1} \mathrm{H}$ NMR $\left(500 \mathrm{MHz}, \mathrm{CD}_{2} \mathrm{Cl}_{2}\right)$ of compound $\mathbf{1 0 b}$

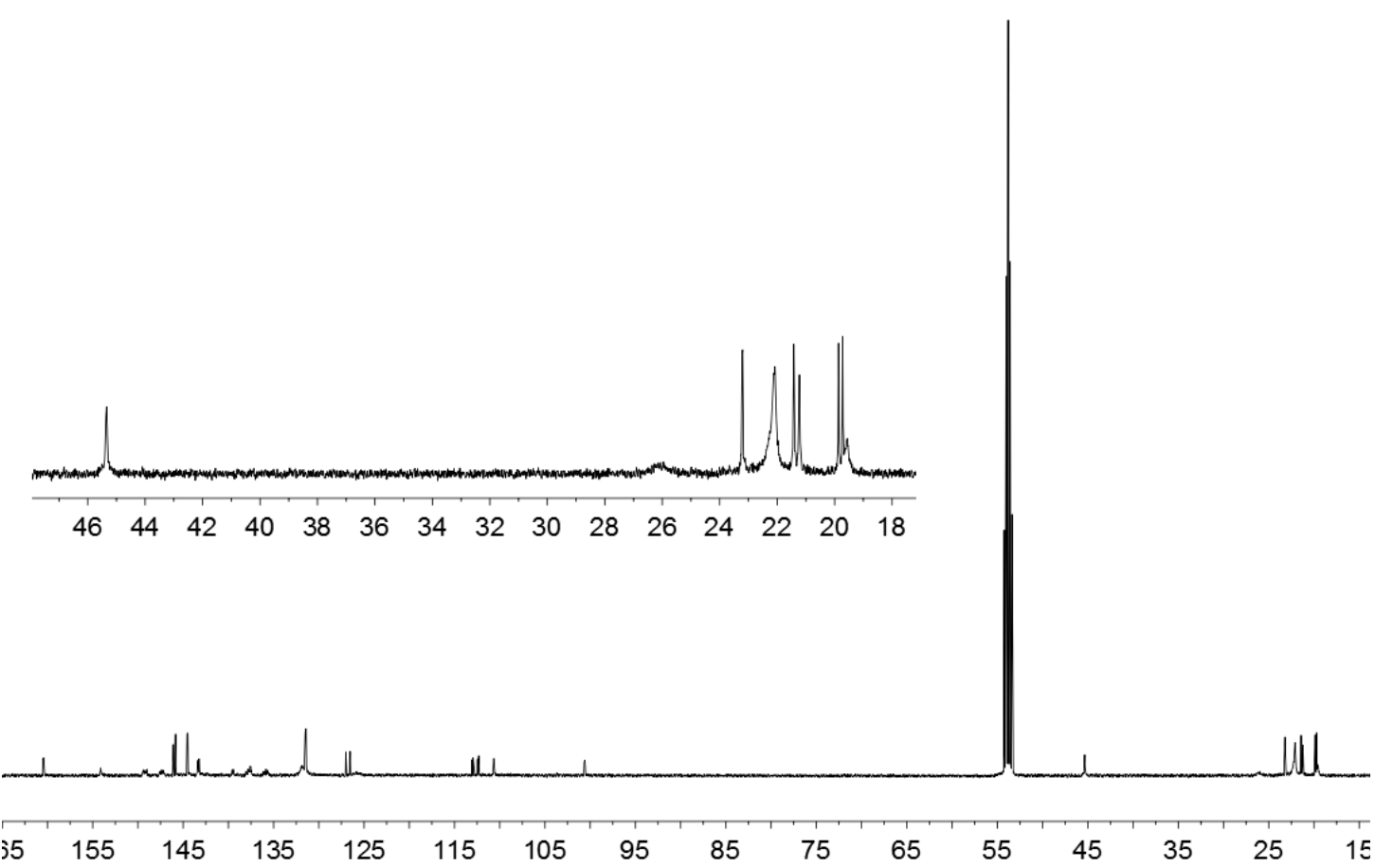

Figure S34. ${ }^{13} \mathbf{C}\left\{{ }^{1} \mathbf{H}\right\}$ NMR $\left(126 \mathrm{MHz}, 299 \mathrm{~K}, \mathrm{CD}_{2} \mathrm{Cl}_{2}\right)$ of compound $\mathbf{1 0 b}$ 


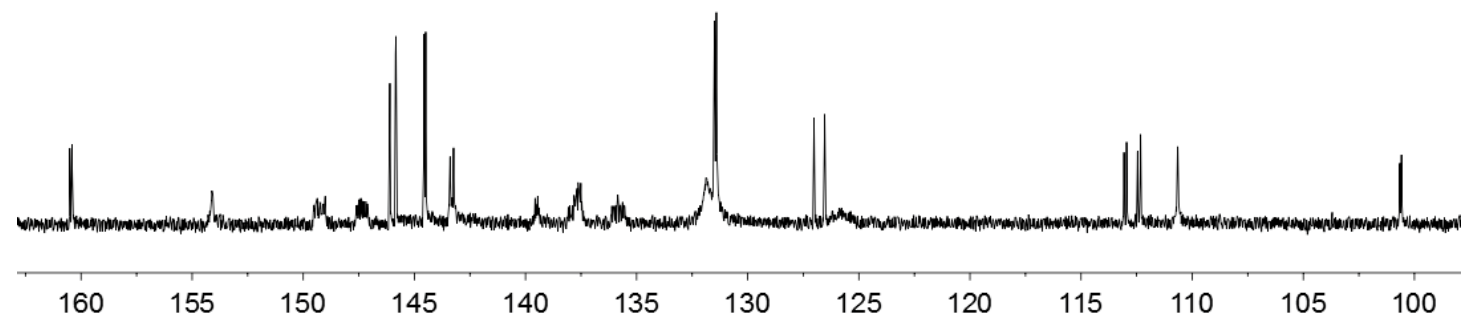

Figure S35. ${ }^{13} \mathbf{C}\left\{{ }^{1} \mathbf{H}\right\}$ NMR $\left(126 \mathrm{MHz}, 299 \mathrm{~K}, \mathrm{CD}_{2} \mathrm{Cl}_{2}\right)$ of compound $\mathbf{1 0 b}$

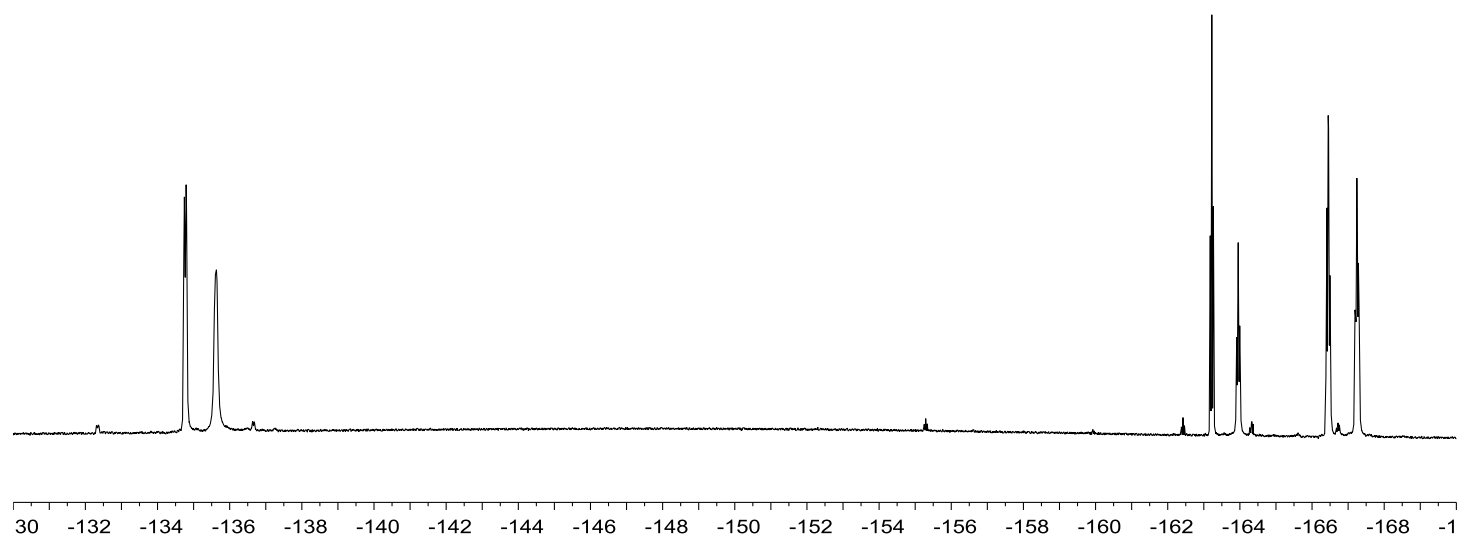

Figure S36. ${ }^{19}$ F NMR $\left(470 \mathrm{MHz}, 299 \mathrm{~K}, \mathrm{CD}_{2} \mathrm{Cl}_{2}\right)$ of compound $\mathbf{1 0 b}$

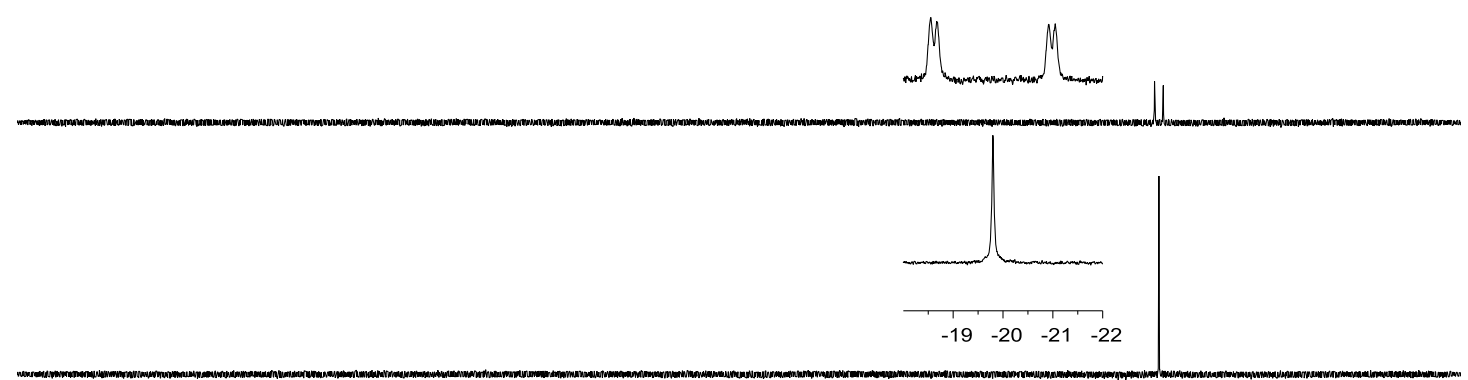

$\begin{array}{lllllllllllllllllllll}300 & 280 & 260 & 240 & 220 & 200 & 180 & 160 & 140 & 120 & 100 & 80 & 60 & 40 & 20 & 0 & -20 & -40 & -60 & -80 & -100\end{array}$

Figure S37. ${ }^{31} \mathbf{P}\left\{{ }^{1} \mathbf{H}\right\}$ and ${ }^{31} \mathbf{P}$ NMR $\left(202 \mathrm{MHz}, 299 \mathrm{~K}, \mathrm{CD}_{2} \mathrm{Cl}_{2}\right)$ of compound $\mathbf{1 0 b}$

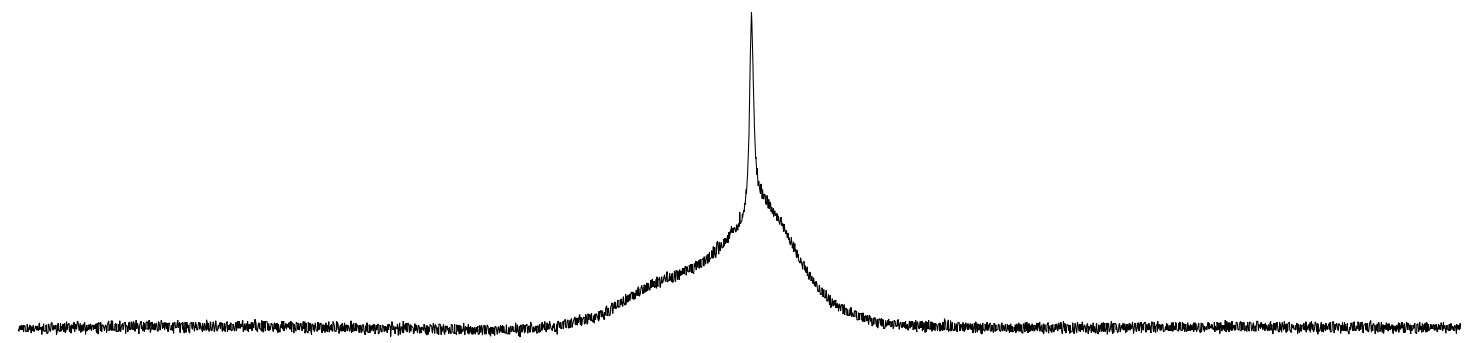

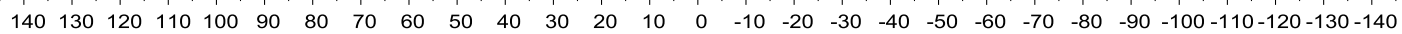

Figure S38. ${ }^{11} \mathbf{B}\left\{{ }^{1} \mathbf{H}\right\}$ NMR $\left(160 \mathrm{MHz}, 299 \mathrm{~K}, \mathrm{CD}_{2} \mathrm{Cl}_{2}\right)$ of compound $\mathbf{1 0 b}$ 


\section{Synthesis of compound 11a}

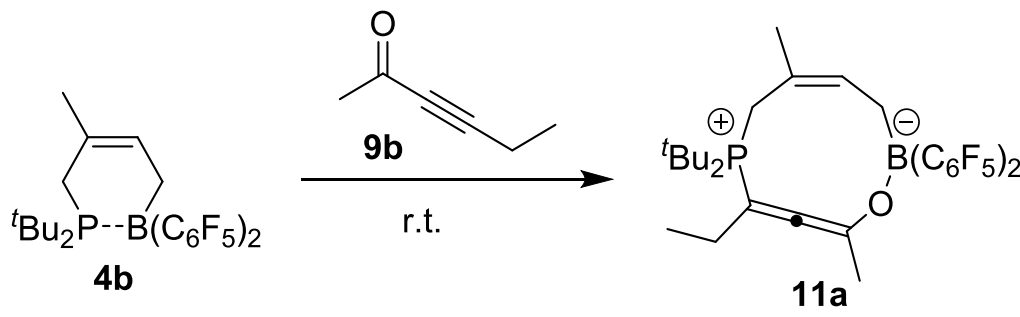

Scheme S6

A solution of compounds $4 \mathbf{b}(111.6 \mathrm{mg}, 0.2 \mathrm{mmol})$ and $9 \mathbf{b}(19.2 \mathrm{mg}, 0.2 \mathrm{mmol})$ in pentane $(2 \mathrm{~mL})$ was stirred at room temperature for $3 \mathrm{~h}$. Then all volatiles were removed in vacuo and the resulting residue was washed with cold pentane $(3 \times 1 \mathrm{~mL})$. After drying in vacuo compound 11a (106.1 mg, 81\%) was obtained as a light yellow solid. Crystals suitable for the X-ray crystal structure analysis were obtained by slow diffusion of pentane to a solution of compound 11a in dichloromethane at $-35^{\circ} \mathrm{C}$. Decomp. $113^{\circ} \mathrm{C}$. Anal. Calc. for $\mathrm{C}_{31} \mathrm{H}_{34} \mathrm{BF}_{10} \mathrm{OP}: \mathrm{C}: 56.90$; H: 5.24. Found: C: 56.43; H: 5.16.

${ }^{1} \mathbf{H}$ NMR $\left(600 \mathrm{MHz}, 299 \mathrm{~K}, \mathrm{CD}_{2} \mathrm{Cl}_{2}\right): \delta=5.89\left(\mathrm{~d},{ }^{4} J_{\mathrm{PH}}=12.9 \mathrm{~Hz}, 1 \mathrm{H},=\mathrm{CH}\right), 3.54(\mathrm{t}$, $\left.{ }^{2} J_{\mathrm{HH}}={ }^{2} J_{\mathrm{PH}}=15.0 \mathrm{~Hz}, 1 \mathrm{H}, \mathrm{PCH}_{2}\right), 2.52\left(\mathrm{dd},{ }^{2} J_{\mathrm{HH}}=15.0 \mathrm{~Hz},{ }^{2} J_{\mathrm{PH}}=12.5 \mathrm{~Hz}, 1 \mathrm{H}, \mathrm{PCH}_{2}\right)$, 2.35, 1.51 (each m, each 1H, $\mathrm{BCH}_{2}$ ), 2.12, 2.02 (each m, each $1 \mathrm{H}, \mathrm{Et}$ ), 1.90 (m, 3H, $\left.\mathrm{CH}_{3}\right), 1.86\left(\mathrm{~d},{ }^{5} \mathrm{~J}_{\mathrm{PH}}=5.5 \mathrm{~Hz}, 3 \mathrm{H},{ }^{\mathrm{O}-{ }^{-}} \mathrm{CH}_{3}\right), 1.58\left(\mathrm{~d},{ }^{3} \mathrm{~J}_{\mathrm{PH}}=13.6 \mathrm{~Hz}, 9 \mathrm{H},{ }^{t} \mathrm{Bu}^{\mathrm{a}}\right), 1.41(\mathrm{~d}$, $\left.{ }^{3} J_{\mathrm{PH}}=14.6 \mathrm{~Hz}, 9 \mathrm{H},{ }^{t} \mathrm{Bu}^{\mathrm{b}}\right), 0.61\left(\mathrm{t},{ }^{3} J_{\mathrm{HH}}=7.1 \mathrm{~Hz}, 3 \mathrm{H}, \mathrm{Et}\right)$.

${ }^{13} \mathbf{C}\left\{{ }^{1} \mathbf{H}\right\}$ NMR $\left(151 \mathrm{MHz}, 299 \mathrm{~K}, \mathrm{CD}_{2} \mathrm{Cl}_{2}\right): \delta=225.7\left(\mathrm{~d},{ }^{2} J_{\mathrm{PC}}=4.3 \mathrm{~Hz},=\mathrm{C}=\right), 139.7(\mathrm{~d}$, $\left.{ }^{3} J_{\mathrm{PC}}=6.7 \mathrm{~Hz},=\mathrm{CH}\right), 135.3\left(\mathrm{~d},{ }^{3} J_{\mathrm{PC}}=12.9 \mathrm{~Hz},=\mathrm{CO}\right), 115.6\left(\mathrm{~d},{ }^{2} J_{\mathrm{PC}}=12.0 \mathrm{~Hz},=\mathrm{C}^{\mathrm{CH} 3}\right)$, $92.7\left(\mathrm{~d},{ }^{1} J_{\mathrm{PC}}=63.1 \mathrm{~Hz},=\mathrm{CP}\right), 37.0\left(\mathrm{~d},{ }^{1} J_{\mathrm{PC}}=31.9 \mathrm{~Hz},{ }^{t} \mathrm{Bu}^{\mathrm{b}}\right), 36.8\left(\mathrm{~d},{ }^{1} J_{\mathrm{PC}}=28.9 \mathrm{~Hz}\right.$, $\left.{ }^{t} \mathrm{Bu}^{\mathrm{a}}\right), 28.9\left({ }^{t} \mathrm{Bu}^{\mathrm{a}}\right), 28.8\left(\mathrm{~d},{ }^{2} J_{\mathrm{PC}}=14.9 \mathrm{~Hz}, \mathrm{Et}\right), 28.1\left({ }^{t} \mathrm{Bu}^{\mathrm{b}}\right), 28.0\left(\mathrm{br}, \mathrm{BCH}_{2}\right), 27.2(\mathrm{~d}$, $\left.{ }^{3} J_{\mathrm{PC}}=4.1 \mathrm{~Hz}, \mathrm{CH}_{3}\right), 25.1\left(\mathrm{~d},{ }^{1} J_{\mathrm{PC}}=43.9 \mathrm{~Hz}, \mathrm{PCH}_{2}\right), 21.2\left(\mathrm{~d},{ }^{4} J_{\mathrm{PC}}=5.2 \mathrm{~Hz},{ }^{\mathrm{O}-\mathrm{C}} \mathrm{CH}_{3}\right)$, $11.3\left(\mathrm{~d},{ }^{3} \mathrm{~J}_{\mathrm{PC}}=4.6 \mathrm{~Hz}, \mathrm{Et}\right),\left[\mathrm{C}_{6} \mathrm{~F}_{5}\right.$ not listed $]$.

${ }^{11} \mathbf{B}\left\{{ }^{1} \mathbf{H}\right\}$ NMR $\left(192 \mathrm{MHz}, 299 \mathrm{~K}, \mathrm{CD}_{2} \mathrm{Cl}_{2}\right): \delta=-1.1\left(v_{1 / 2} \sim 80 \mathrm{~Hz}\right)$.

${ }^{31} \mathbf{P}\left\{{ }^{1} \mathbf{H}\right\} \mathbf{N M R}\left(243 \mathrm{MHz}, 299 \mathrm{~K}, \mathrm{CD}_{2} \mathrm{Cl}_{2}\right): \delta=31.8\left(v_{1 / 2} \sim 2 \mathrm{~Hz}\right)$.

${ }^{19}$ F NMR $\left(564 \mathrm{MHz}, 299 \mathrm{~K}, \mathrm{CD}_{2} \mathrm{Cl}_{2}\right): \delta=-133.0(\mathrm{~m}, 2 \mathrm{~F}, o),-162.9\left(\mathrm{t},{ }^{3} J_{\mathrm{FF}}=20.2 \mathrm{~Hz}\right.$, $1 \mathrm{~F}, p),-167.1(\mathrm{~m}, 2 \mathrm{~F}, m)\left(\mathrm{C}_{6} \mathrm{~F}_{5}\right)\left[\Delta \delta^{19} \mathrm{~F}_{\mathrm{mp}}=4.2\right],-134.8(\mathrm{~m}, 2 \mathrm{~F}, o),-163.5\left(\mathrm{t},{ }^{3} J_{\mathrm{FF}}=20.2\right.$ $\mathrm{Hz}, 1 \mathrm{~F}, p),-166.9(\mathrm{~m}, 2 \mathrm{~F}, m)\left(\mathrm{C}_{6} \mathrm{~F}_{5}\right)\left[\Delta \delta^{19} \mathrm{~F}_{\mathrm{mp}}=3.4\right]$.

${ }^{1} \mathbf{H},{ }^{1} \mathrm{H}$-GCOSY $\left(600 \mathrm{MHz} / 600 \mathrm{MHz}, 299 \mathrm{~K}, \mathrm{CD}_{2} \mathrm{Cl}_{2}\right)$ [selected traces]: $\delta{ }^{1} \mathrm{H} / \delta^{1} \mathrm{H}=$ $5.89 / 1.90\left(=\mathrm{CH} / \mathrm{CH}_{3}\right), 3.54$ / $2.52\left(\mathrm{PCH}_{2} / \mathrm{PCH}_{2}\right), 2.35 / 1.51\left(\mathrm{BCH}_{2} / \mathrm{BCH}_{2}\right), 2.12 /$ 2.02, $0.61(\mathrm{Et} / \mathrm{Et}, \mathrm{Et})$.

${ }^{1} \mathbf{H},{ }^{13} \mathbf{C}-G H S Q C\left(600 \mathrm{MHz} / 151 \mathrm{MHz}, 299 \mathrm{~K}, \mathrm{CD}_{2} \mathrm{Cl}_{2}\right): \delta{ }^{1} \mathrm{H} / \delta{ }^{13} \mathrm{C}=5.89 / 139.7$ (=CH), 3.54, 2.52 / $25.1\left(\mathrm{PCH}_{2}\right), 2.35,1.51 / 28.0\left(\mathrm{BCH}_{2}\right), 2.12,2.02$ / 28.8 (Et), 1.90 / $27.2\left(\mathrm{CH}_{3}\right), 1.86 / 21.2\left({ }^{\mathrm{O}-\mathrm{C}} \mathrm{CH}_{3}\right), 1.58 / 28.9\left({ }^{t} \mathrm{Bu}^{\mathrm{a}}\right), 1.41 / 28.1\left({ }^{t} \mathrm{Bu}^{\mathrm{b}}\right), 0.61 / 11.3(\mathrm{Et})$.

${ }^{\mathbf{1}} \mathbf{H},{ }^{13} \mathbf{C}-\mathbf{G H M B C}\left(600 \mathrm{MHz} / 151 \mathrm{MHz}, 299 \mathrm{~K}, \mathrm{CD}_{2} \mathrm{Cl}_{2}\right)$ [selected traces]: $\delta{ }^{1} \mathrm{H} / \delta{ }^{13} \mathrm{C}$ $=3.54,2.52$, 2.35, $1.90 / 115.6\left(\mathrm{PCH}_{2}, \mathrm{PCH}_{2}, \mathrm{BCH}_{2}, \mathrm{CH}_{3} /=\mathrm{C}^{\mathrm{CH}} 3\right), 2.52,2.12,2.02$, 
0.61 /92.7 $\left(\mathrm{PCH}_{2}\right.$, Et, Et, Et / =CP), 2.12, 2.02, $1.86 / 225.7\left(\mathrm{Et}, \mathrm{Et},{ }^{\mathrm{O}-\mathrm{C}} \mathrm{CH}_{3} /=\mathrm{C}=\right), 1.86$ / $135.3\left({ }^{\mathrm{O}-\mathrm{C}} \mathrm{CH}_{3} /=\mathrm{CO}\right), 1.58 / 36.8\left({ }^{t} \mathrm{Bu}^{\mathrm{a}} /{ }^{t} \mathrm{Bu}^{\mathrm{a}}\right), 1.41 / 37.0\left({ }^{t} \mathrm{Bu}^{\mathrm{b}} /{ }^{t} \mathrm{Bu}^{\mathrm{b}}\right)$.

${ }^{19} \mathbf{F},{ }^{19} \mathrm{~F}-G C O S Y\left(564 \mathrm{MHz} / 564 \mathrm{MHz}, 299 \mathrm{~K}, \mathrm{CD}_{2} \mathrm{Cl}_{2}\right): \delta{ }^{19} \mathrm{~F} / \delta{ }^{19} \mathrm{~F}=-167.1 /-133.0$, $-162.9\left(m-\mathrm{C}_{6} \mathrm{~F}_{5}^{\mathrm{a}} / o-\mathrm{C}_{6} \mathrm{~F}_{5}^{\mathrm{a}}, p-\mathrm{C}_{6} \mathrm{~F}_{5}^{\mathrm{a}}\right),-166.9 /-134.8,-163.5\left(m-\mathrm{C}_{6} \mathrm{~F}_{5}^{\mathrm{b}} / o-\mathrm{C}_{6} \mathrm{~F}_{5}{ }^{\mathrm{b}}, p-\mathrm{C}_{6} \mathrm{~F}_{5}^{\mathrm{b}}\right)$.

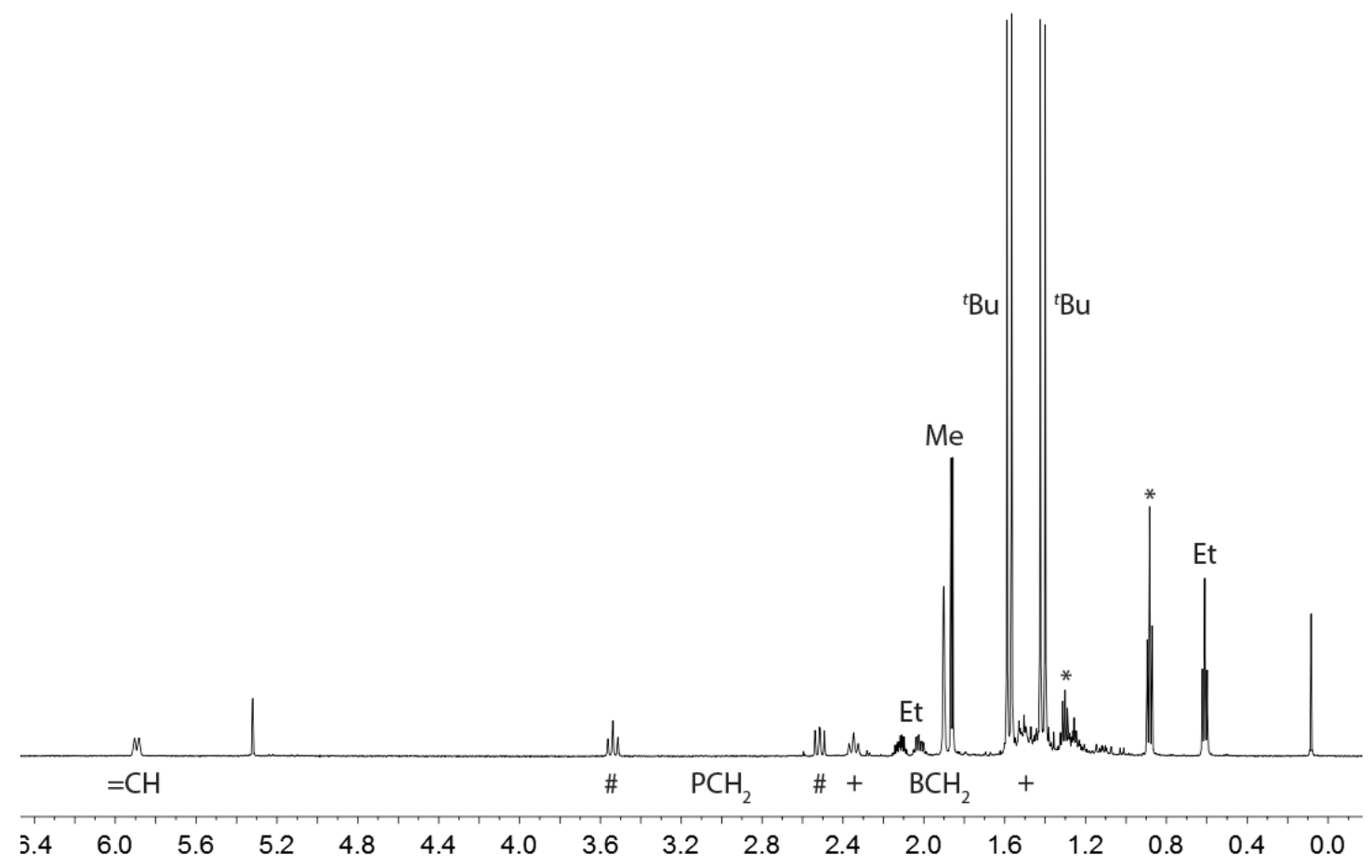

Figure S39. ${ }^{1} \mathbf{H}$ NMR $\left(600 \mathrm{MHz}, 299 \mathrm{~K}, \mathrm{CD}_{2} \mathrm{Cl}_{2}\right)$ of compound 11a [admixed with pentane $(*)]$

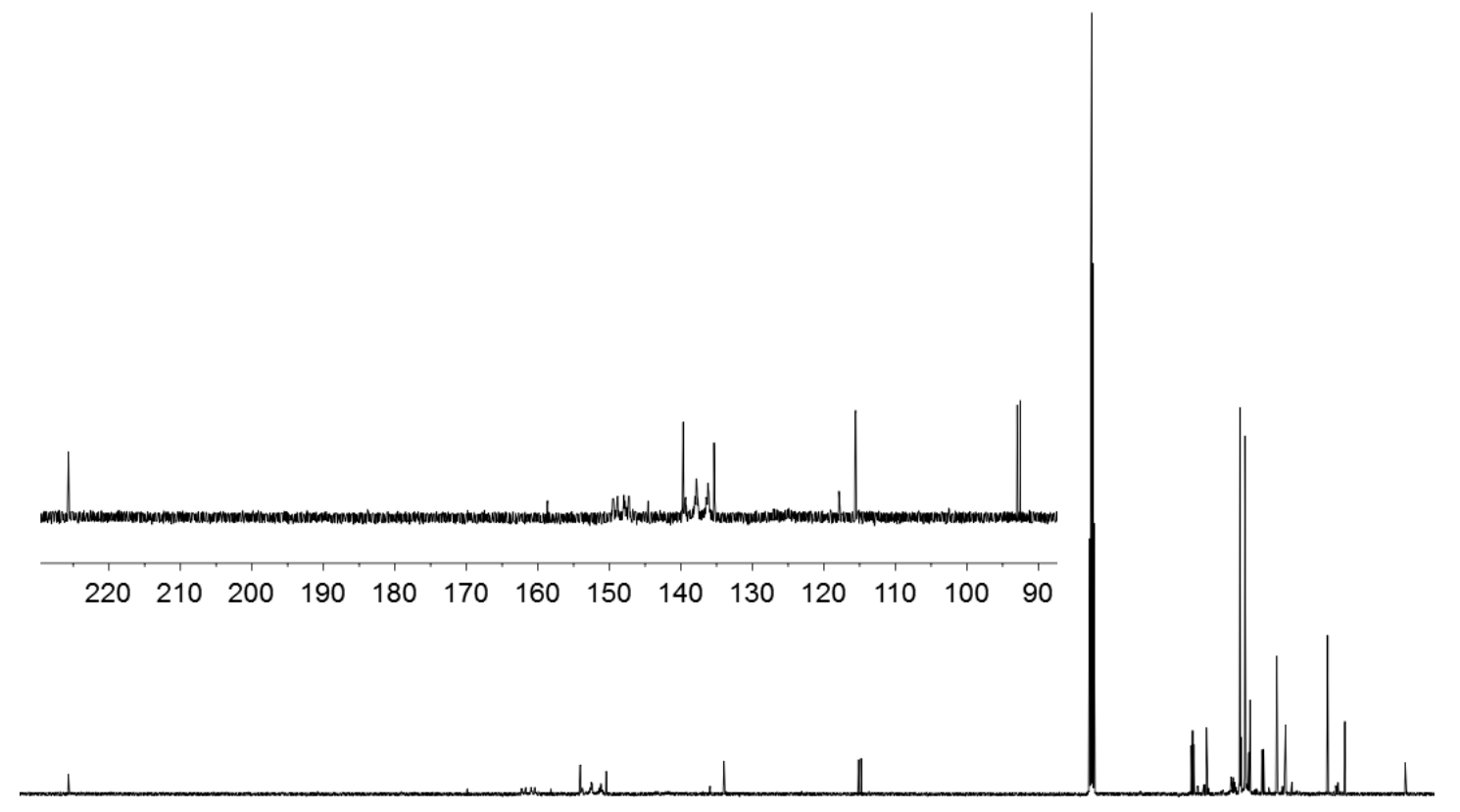

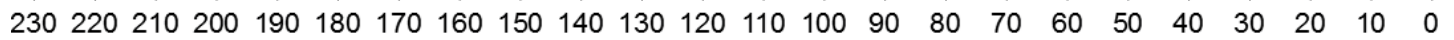

Figure S40. ${ }^{13} \mathbf{C}\left\{{ }^{1} \mathbf{H}\right\}$ NMR $\left(151 \mathrm{MHz}, 299 \mathrm{~K}, \mathrm{CD}_{2} \mathrm{Cl}_{2}\right)$ of compound 11a [admixed with pentane] 


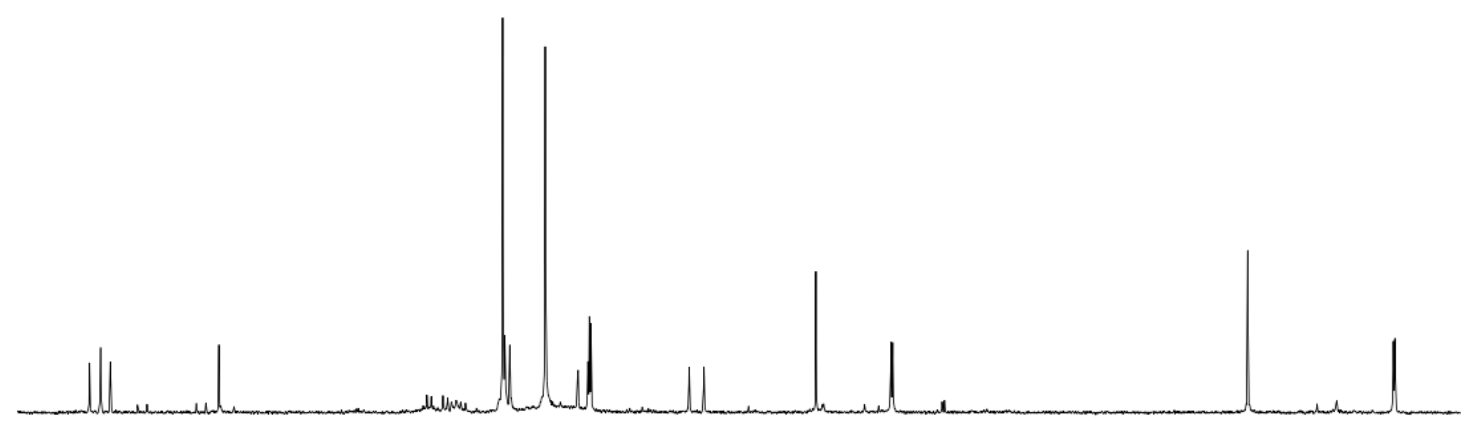

$\begin{array}{lllllllllllllllllllllllllllll}38 & 37 & 36 & 35 & 34 & 33 & 32 & 31 & 30 & 29 & 28 & 27 & 26 & 25 & 24 & 23 & 22 & 21 & 20 & 19 & 18 & 17 & 16 & 15 & 14 & 13 & 12 & 11 & 1\end{array}$

Figure S41. ${ }^{13} \mathbf{C}\left\{{ }^{1} \mathbf{H}\right\}$ NMR $\left(151 \mathrm{MHz}, 299 \mathrm{~K}, \mathrm{CD}_{2} \mathrm{Cl}_{2}\right)$ of compound 11a [admixed with pentane]

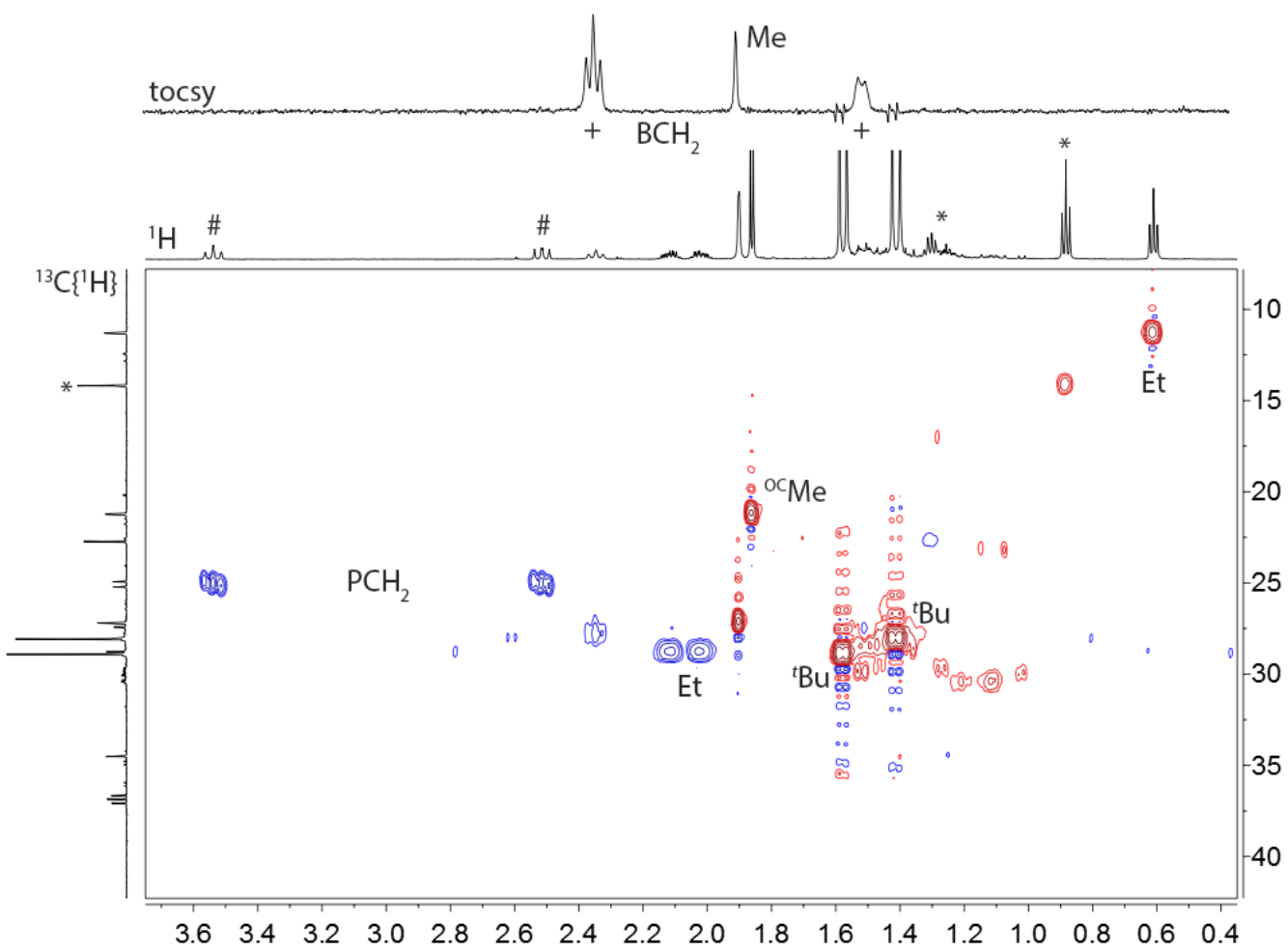

Figure S42. ${ }^{1} \mathbf{H},{ }^{13} \mathbf{C}-\mathbf{G H S Q C}\left(600 \mathrm{MHz} / 151 \mathrm{MHz}, 299 \mathrm{~K}, \mathrm{CD}_{2} \mathrm{Cl}_{2}\right)$ of compound $11 \mathrm{a}$ [admixed with pentane $(*)$ ]. ${ }^{1} \mathrm{H},{ }^{1} \mathrm{H}$ tocsy: irradiation at $\delta^{1} \mathrm{H}_{\mathrm{irr}}=2.35\left(\mathrm{BCH}_{2}\right)$. 


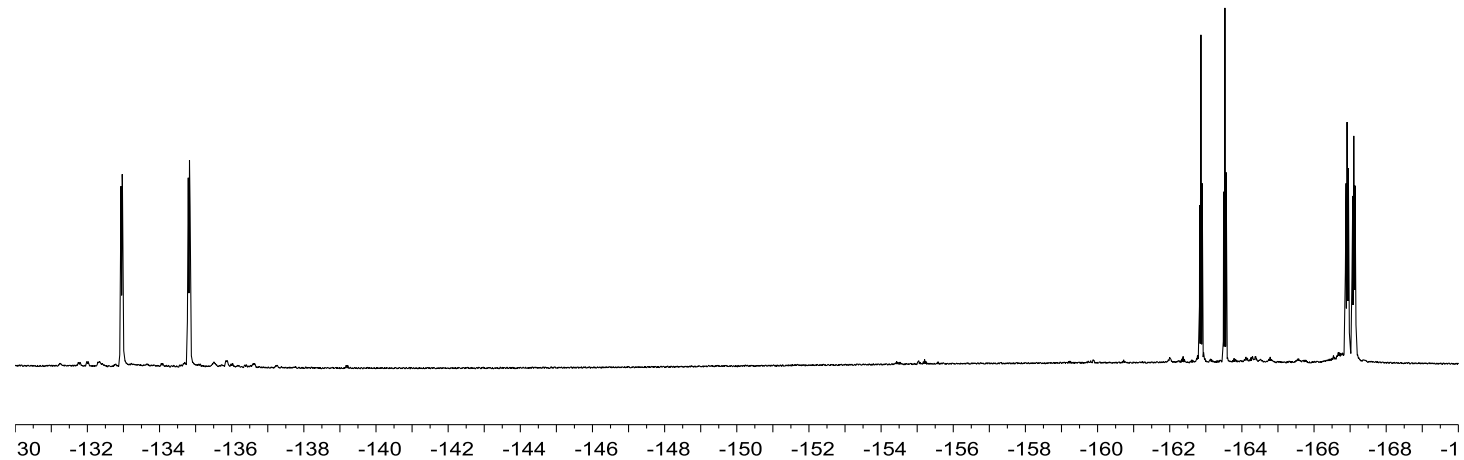

Figure S43. ${ }^{19}$ F NMR $\left(564 \mathrm{MHz}, 299 \mathrm{~K}, \mathrm{CD}_{2} \mathrm{Cl}_{2}\right)$ of compound 11a

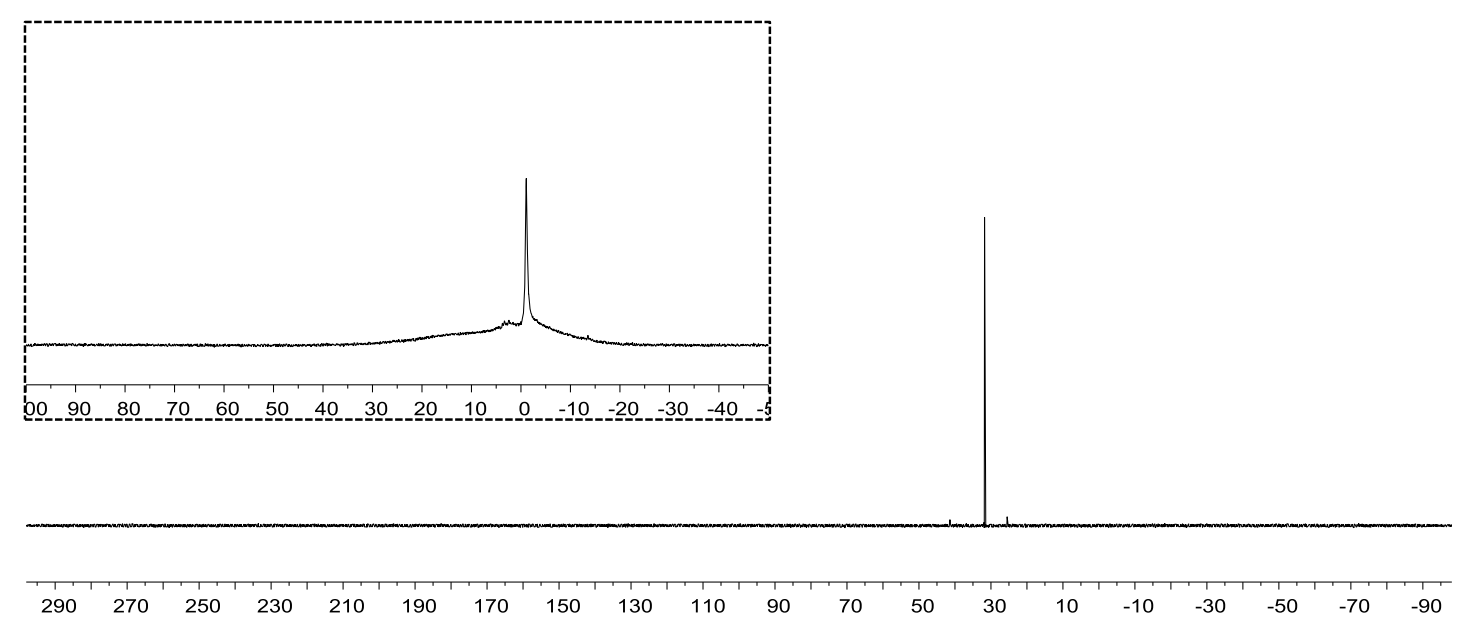

Figure S44. ${ }^{11} \mathbf{B}\left\{{ }^{1} \mathbf{H}\right\}$ NMR $\left(192 \mathrm{MHz}, 299 \mathrm{~K}, \mathrm{CD}_{2} \mathrm{Cl}_{2}\right)$ and ${ }^{31} \mathbf{P}\left\{{ }^{1} \mathbf{H}\right\} \mathbf{N M R}(243 \mathrm{MHz}$, $299 \mathrm{~K}, \mathrm{CD}_{2} \mathrm{Cl}_{2}$ ) of compound $\mathbf{1 1 a}$

X-ray crystal structure analysis of compound 11a: formula $\mathrm{C}_{31} \mathrm{H}_{34} \mathrm{BF} 10 \mathrm{OP}, M=$ 654.36, colourless crystal, $0.18 \times 0.15 \times 0.15 \mathrm{~mm}, a=9.1467(2), b=14.6625(3), c=$ 14.7129(4) $\AA, \alpha=90.266(1), \beta=102.873(1), \gamma=107.489(1)^{\circ}, V=1829.2(1) \AA^{3}, \rho_{\text {calc }}$ $=1.188 \mathrm{gcm}^{-3}, \mu=0.147 \mathrm{~mm}^{-1}$, empirical absorption correction $(0.974 \leq \mathrm{T} \leq 0.978), Z$ $=2$, triclinic, space group $P^{-} \overline{1}$ (No. 2$), \lambda=0.71073 \AA, T=223(2) \mathrm{K}, \omega$ and $\varphi$ scans, 17742 reflections collected $( \pm h, \pm k, \pm l), 6342$ independent $\left(R_{\text {int }}=0.059\right)$ and 4693 observed reflections $[I>2 \sigma(I)], 400$ refined parameters, $R=0.086, w R^{2}=0.239$, max. (min.) residual electron density $0.37(-0.33)$ e. $\AA^{-3}$, hydrogen atoms were calculated and refined as riding atoms. 


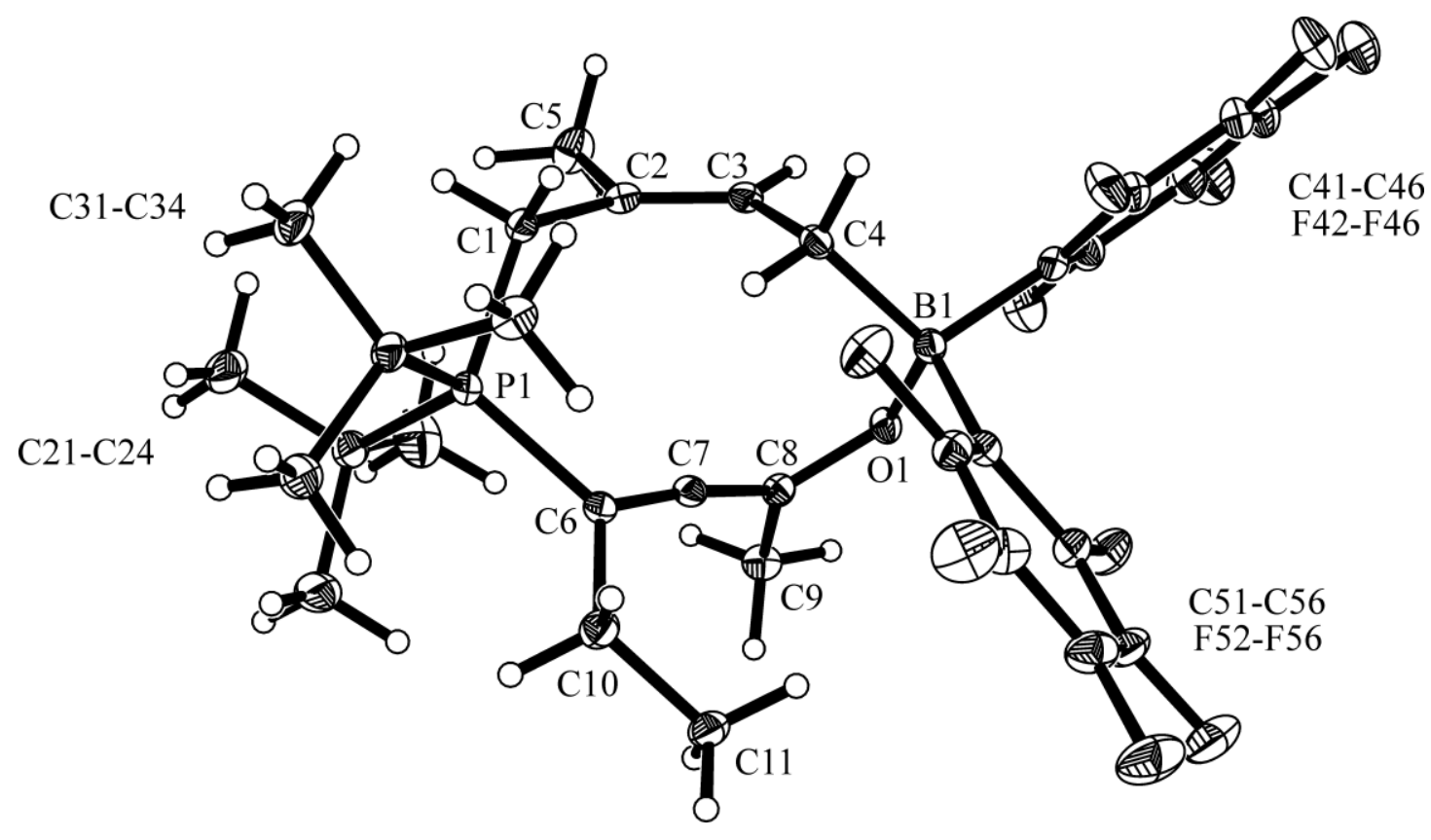

Figure S45. 


\section{Control experiments (4b + 9b: NMR scale)}

Reactions of compounds $\mathbf{4 b}$ with alkyne $\mathbf{9 b}$ monitored by NMR spectroscopy

Alkyne 9b (0.03 mmol, $2.9 \mathrm{mg})$ was added to a solution of compound $\mathbf{4 b}(0.03 \mathrm{mmol}$, $16.7 \mathrm{mg})$ in $\mathrm{CD}_{2} \mathrm{Cl}_{2}(0.5 \mathrm{~mL})$ [alternatively with $\mathrm{C}_{6} \mathrm{D}_{6}(0.5 \mathrm{~mL})$ ]. The reaction mixture was transferred to a NMR tube and monitored by NMR spectroscopy.

Comment: NMR spectra showed that the reaction of compound $\mathbf{4 b}$ with the alkyne 9b was finished in less than 10 min. at r.t. after mixing the components to give compound 11a in quantitative yield.

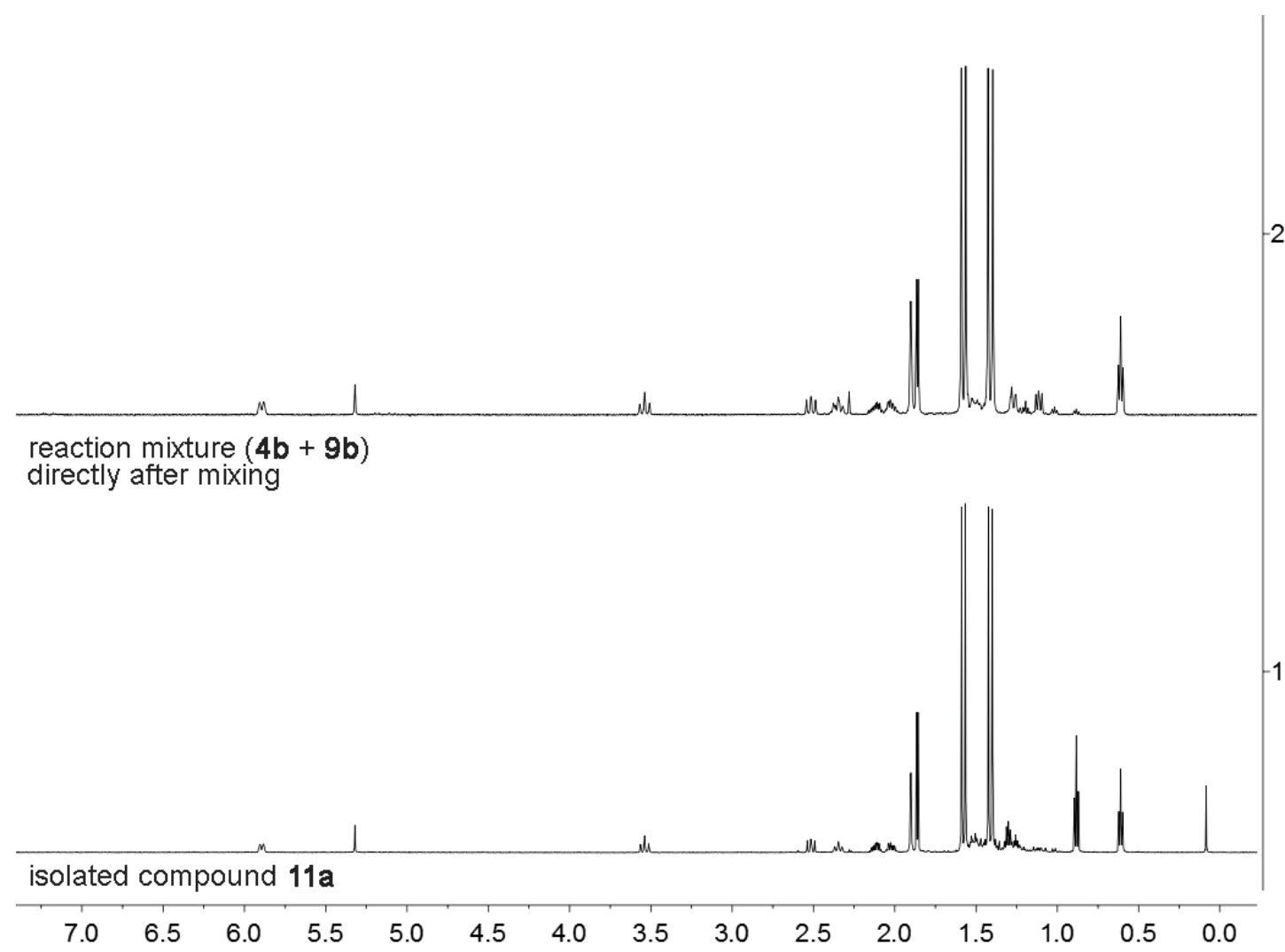

Figure S46. (1) ${ }^{1} \mathbf{H}$ NMR (600 MHz, $\left.299 \mathrm{~K}, \mathrm{CD}_{2} \mathrm{Cl}_{2}\right)$ spectrum of isolated compound 11a and (2) ${ }^{1} \mathbf{H}$ NMR $\left(500 \mathrm{MHz}, 299 \mathrm{~K}, \mathrm{CD}_{2} \mathrm{Cl}_{2}\right.$ ) spectrum of the reaction mixture of the reaction of compounds $\mathbf{4 b}$ with $9 \mathrm{~b}$ 


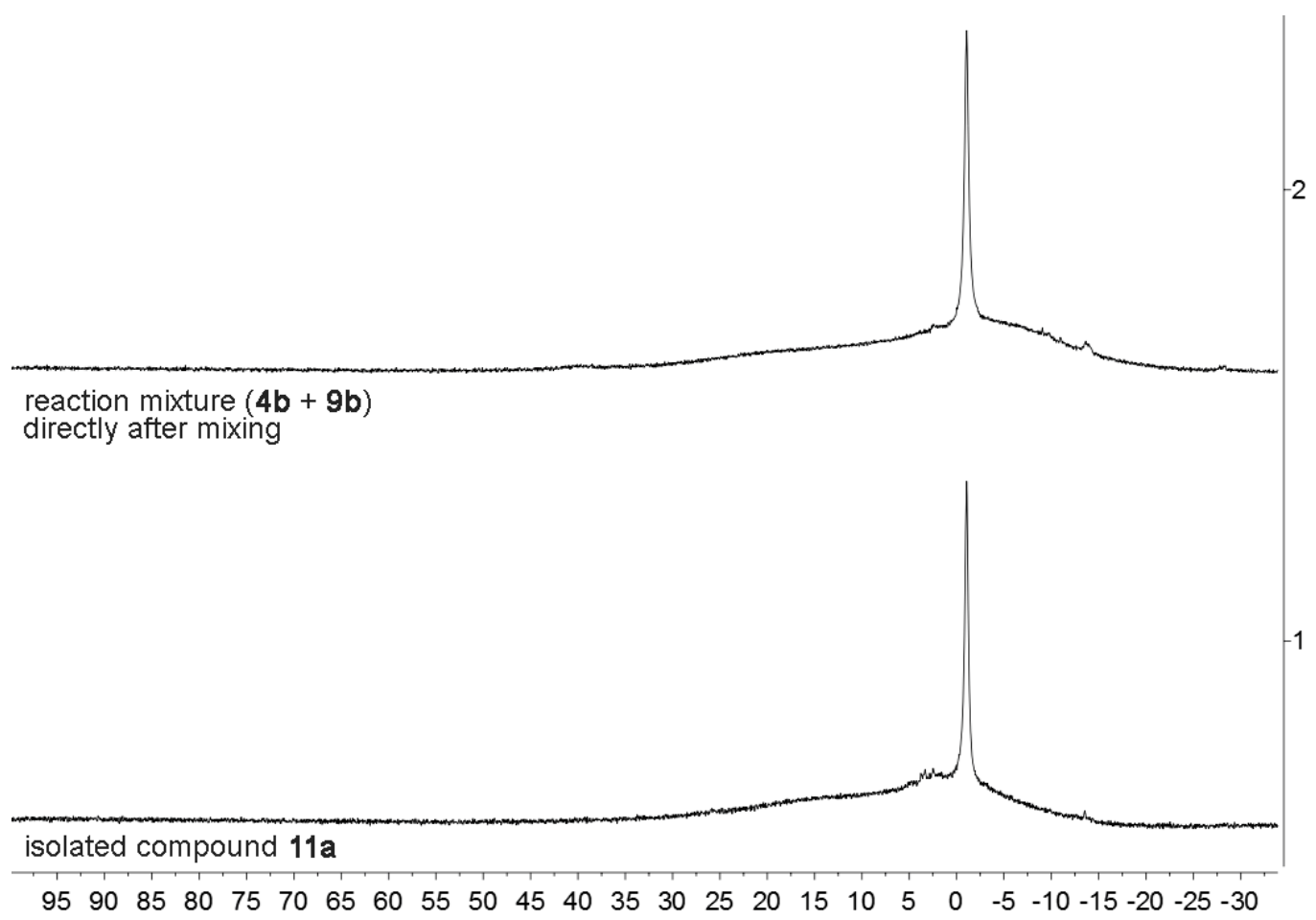

Figure S47. (1) ${ }^{11} \mathbf{B}\left\{{ }^{1} \mathbf{H}\right\} \mathbf{N M R}\left(192 \mathrm{MHz}, 299 \mathrm{~K}, \mathrm{CD}_{2} \mathrm{Cl}_{2}\right)$ spectrum of isolated compound 11a and (2) ${ }^{11} \mathbf{B}\left\{{ }^{1} \mathbf{H}\right\} \mathbf{N M R}\left(160 \mathrm{MHz}, 299 \mathrm{~K}, \mathrm{CD}_{2} \mathrm{Cl}_{2}\right)$ spectrum of the reaction mixture of the reaction of compounds $\mathbf{4 b}$ with $\mathbf{9 b}$

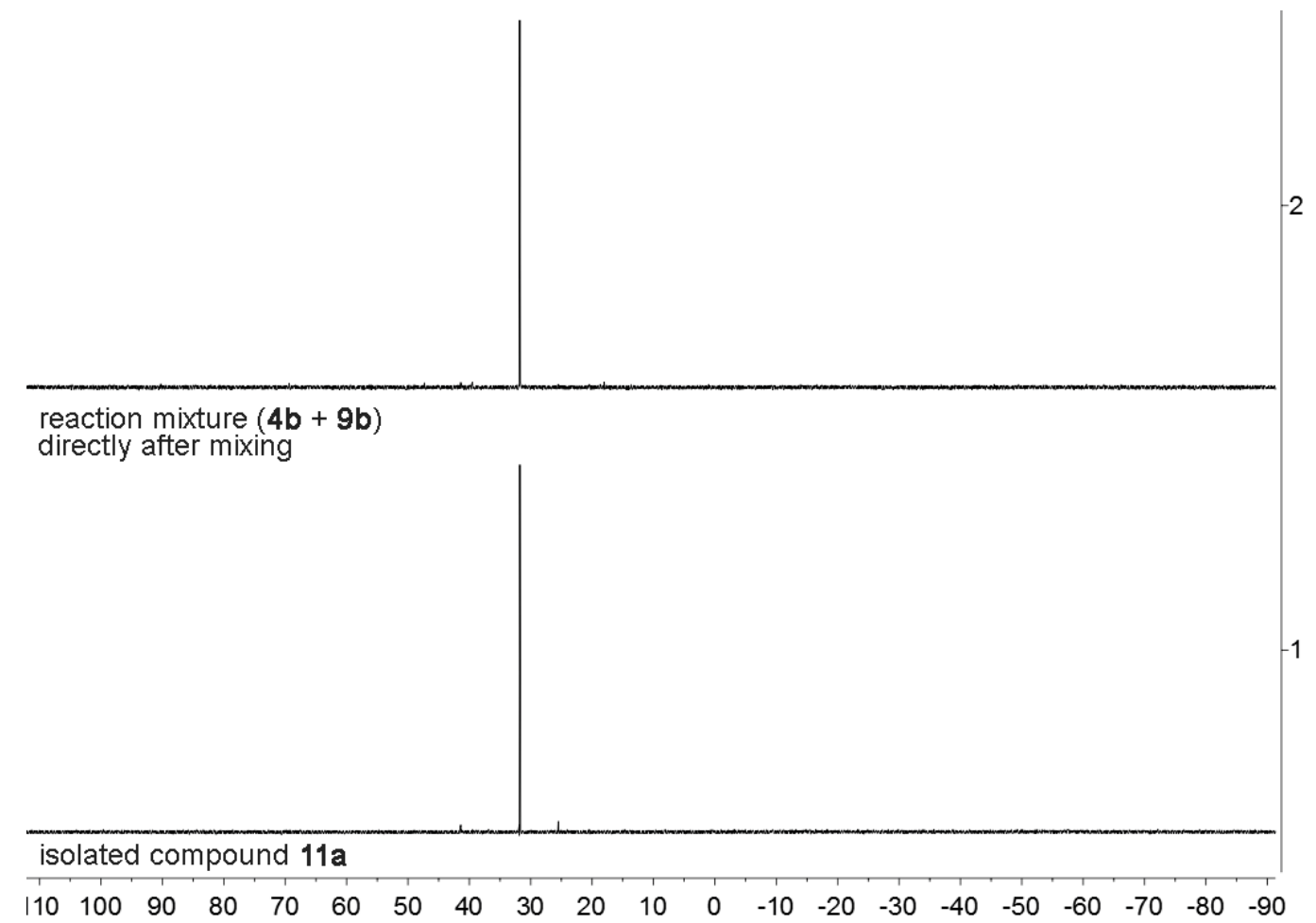

Figure S48. (1) ${ }^{31} \mathbf{P}\left\{{ }^{1} \mathbf{H}\right\} \mathbf{N M R}\left(243 \mathrm{MHz}, 299 \mathrm{~K}, \mathrm{CD}_{2} \mathrm{Cl}_{2}\right)$ spectrum of isolated compound 11a and (2) ${ }^{31} \mathbf{P}\left\{{ }^{1} \mathbf{H}\right\} \mathbf{N M R}\left(202 \mathrm{MHz}, 299 \mathrm{~K}, \mathrm{CD}_{2} \mathrm{Cl}_{2}\right)$ spectrum of the reaction mixture of the reaction of compounds $4 \mathbf{b}$ with $9 b$ 


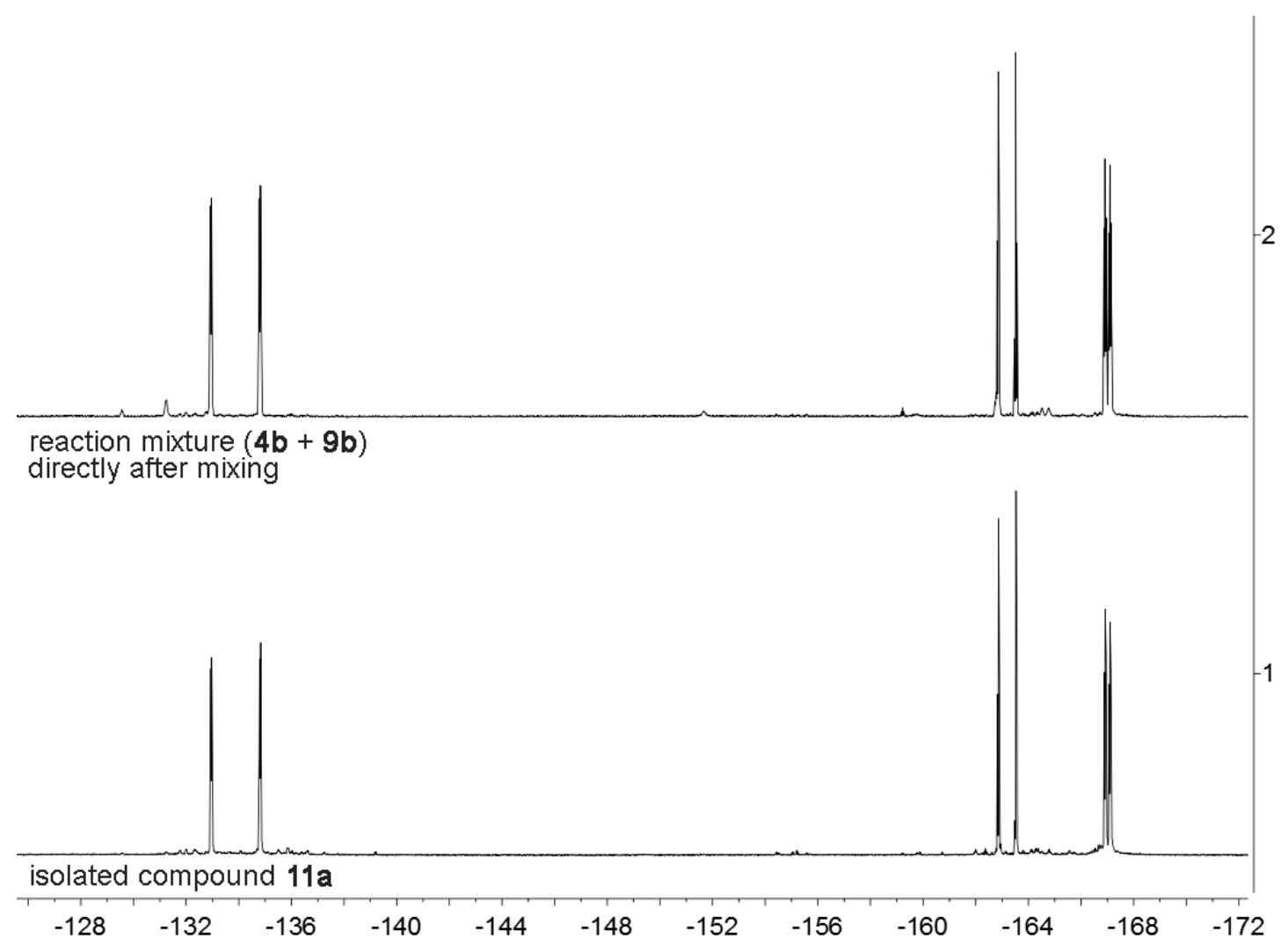

Figure S49. (1) ${ }^{19} \mathrm{FNMR}\left(564 \mathrm{MHz}, 299 \mathrm{~K}, \mathrm{CD}_{2} \mathrm{Cl}_{2}\right.$ ) spectrum of isolated compound 11a and (2) ${ }^{19} \mathrm{FNMR}\left(470 \mathrm{MHz}, 299 \mathrm{~K}, \mathrm{CD}_{2} \mathrm{Cl}_{2}\right)$ spectrum of the reaction mixture of the reaction of compounds $\mathbf{4 b}$ with $\mathbf{9 b}$ 


\section{Synthesis of compound 12}

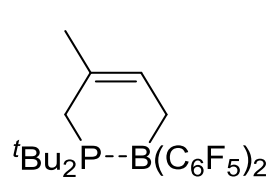

4b

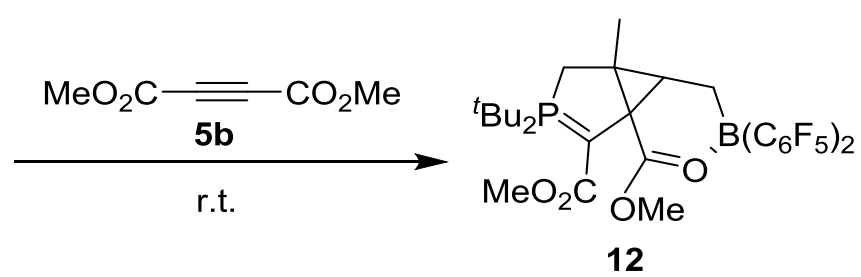

Scheme S7

A solution of compounds $\mathbf{4 b}(111.6 \mathrm{mg}, 0.2 \mathrm{mmol})$ and $\mathbf{5 b}(28.4 \mathrm{mg}, 0.2 \mathrm{mmol})$ in pentane $(2 \mathrm{~mL})$ was stirred at room temperature for $3 \mathrm{~h}$. Then all volatiles were removed in vacuo and the resulting residue was washed with cold pentane $(3 \times 1 \mathrm{~mL})$. After drying in vacuo compound $12(119.6 \mathrm{mg}, 85 \%)$ was obtained as a light yellow solid (two isomers 85:15 $\left.\left({ }^{1} \mathrm{H}\right)\right)$. Crystals suitable for the X-ray crystal structure analysis were obtained by slow diffusion of pentane to a solution of compound $\mathbf{1 2}$ in dichloromethane at $-35{ }^{\circ} \mathrm{C}$. Decomp. $201{ }^{\circ} \mathrm{C}$. Anal. Calc. for $\mathrm{C}_{31} \mathrm{H}_{32} \mathrm{BF}_{10} \mathrm{O}_{4} \mathrm{P}: \mathrm{C}: 53.16 ; \mathrm{H}: 4.61$. Found: C: 53.29; H: 4.61.

${ }^{1} \mathbf{H}$ NMR $\left(500 \mathrm{MHz}, 299 \mathrm{~K}, \mathrm{CD}_{2} \mathrm{Cl}_{2}\right): \delta=$ major isomer $(85 \mathrm{~mol} \%): 4.07\left(\mathrm{~s}, 3 \mathrm{H}, \mathrm{OCH}_{3}{ }^{\mathrm{B}}\right)$, $2.88\left(\mathrm{~s}, 3 \mathrm{H}, \mathrm{OCH}_{3}\right), 2.34\left(\mathrm{dd},{ }^{2} J_{\mathrm{HH}}=15.8 \mathrm{~Hz},{ }^{2} J_{\mathrm{PH}}=5.9 \mathrm{~Hz}, 1 \mathrm{H}, \mathrm{PCH}_{2}\right), 2.18\left(\mathrm{dd},{ }^{2} J_{\mathrm{HH}}\right.$ $\left.=15.8 \mathrm{~Hz},{ }^{2} J_{\mathrm{PH}}=10.3 \mathrm{~Hz}, 1 \mathrm{H}, \mathrm{PCH}_{2}\right), 1.97(\mathrm{~m}, 1 \mathrm{H}, \mathrm{CH}), 1.96,0.87$ (each m, each $1 \mathrm{H}$, $\left.\mathrm{BCH}_{2}\right), 1.40\left(\mathrm{~d},{ }^{3} J_{\mathrm{PH}}=14.7 \mathrm{~Hz}, 9 \mathrm{H},{ }^{t} \mathrm{Bu}^{\mathrm{a}}\right), 1.39\left(\mathrm{~d},{ }^{3} J_{\mathrm{PH}}=14.9 \mathrm{~Hz}, 9 \mathrm{H},{ }^{t} \mathrm{Bu}^{\mathrm{b}}\right), 1.26(\mathrm{~s}$, $\left.3 \mathrm{H}, \mathrm{CH}_{3}\right)$; minor isomer $(15 \mathrm{~mol} \%)$ : $4.07\left(\mathrm{~s}, 3 \mathrm{H}, \mathrm{OCH}_{3}{ }^{\mathrm{B}}\right), 3.28\left(\mathrm{~s}, 3 \mathrm{H}, \mathrm{OCH}_{3}\right), 2.43(\mathrm{dd}$, $\left.{ }^{2} J_{\mathrm{HH}}=15.8 \mathrm{~Hz},{ }^{2} J_{\mathrm{PH}}=5.9 \mathrm{~Hz}, 1 \mathrm{H}, \mathrm{PCH}_{2}\right), 2.26\left(\mathrm{dd},{ }^{2} J_{\mathrm{HH}}=15.8 \mathrm{~Hz},{ }^{2} J_{\mathrm{PH}}=10.2 \mathrm{~Hz}, 1 \mathrm{H}\right.$, $\left.\mathrm{PCH}_{2}\right), 1.89(\mathrm{~m}, 1 \mathrm{H}, \mathrm{CH}), 1.88,0.84$ (each m, each $\left.1 \mathrm{H}, \mathrm{BCH}_{2}\right), 1.35\left(\mathrm{~d},{ }^{3} \mathrm{~J}_{\mathrm{PH}}=14.6 \mathrm{~Hz}\right.$, $\left.9 \mathrm{H},{ }^{t} \mathrm{Bu}^{\mathrm{a}}\right), 1.31\left(\mathrm{~d},{ }^{3} \mathrm{~J}_{\mathrm{PH}}=14.8 \mathrm{~Hz}, 9 \mathrm{H},{ }^{t} \mathrm{Bu}^{\mathrm{b}}\right), 1.25\left(\mathrm{~s}, 3 \mathrm{H}, \mathrm{CH}_{3}\right)$.

${ }^{13} \mathbf{C}\left\{{ }^{1} \mathbf{H}\right\}$ NMR $\left(126 \mathrm{MHz}, 299 \mathrm{~K}, \mathrm{CD}_{2} \mathrm{Cl}_{2}\right): \delta=$ major isomer: $187.0\left(\mathrm{~d},{ }^{3} J_{\mathrm{PC}}=8.2 \mathrm{~Hz}\right.$, $\mathrm{C}=\mathrm{OB}), 168.2\left(\mathrm{~d},{ }^{2} J_{\mathrm{PC}}=25.2 \mathrm{~Hz}, \mathrm{C}=\mathrm{O}\right), 57.34\left(\mathrm{OCH}_{3}{ }^{\mathrm{B}}\right), 49.3\left(\mathrm{~d},{ }^{4} J_{\mathrm{PC}}=1.7 \mathrm{~Hz}, \mathrm{OCH}_{3}\right)$, $48.4\left(\mathrm{~d},{ }^{1} J_{\mathrm{PC}}=113.0 \mathrm{~Hz}, \mathrm{C}=\mathrm{P}\right), 42.40(\mathrm{CH}), 41.1\left(\mathrm{~d},{ }^{2} J_{\mathrm{PC}}=21.6 \mathrm{~Hz},>\mathrm{C}<\right), 40.5\left(\mathrm{~d},{ }^{2} J_{\mathrm{PC}}\right.$ $\left.=0.8 \mathrm{~Hz}, \mathrm{C}^{\mathrm{Me}}\right), 38.8\left(\mathrm{~d},{ }^{1} J_{\mathrm{PC}}=35.1 \mathrm{~Hz},{ }^{t} \mathrm{Bu}^{\mathrm{a}}\right), 35.3\left(\mathrm{~d},{ }^{1} J_{\mathrm{PC}}=42.7 \mathrm{~Hz},{ }^{t} \mathrm{Bu}^{\mathrm{b}}\right), 30.5(\mathrm{~d}$, $\left.{ }^{1} J_{\mathrm{PC}}=53.1 \mathrm{~Hz}, \mathrm{PCH}_{2}\right), 28.6\left(\mathrm{~d},{ }^{2} J_{\mathrm{PC}}=1.0 \mathrm{~Hz},{ }^{t} \mathrm{Bu}^{\mathrm{b}}\right), 27.4\left(\mathrm{~d},{ }^{2} J_{\mathrm{PC}}=1.7 \mathrm{~Hz},{ }^{t} \mathrm{Bu}^{\mathrm{a}}\right), 16.4$ $\left(\mathrm{d},{ }^{3} J_{\mathrm{PC}}=6.3 \mathrm{~Hz}, \mathrm{CH}_{3}\right), 14.7\left(\mathrm{br}, \mathrm{BCH}_{2}\right)$; minor isomer: $186.4\left(\mathrm{~d},{ }^{3} J_{\mathrm{PC}}=8.2 \mathrm{~Hz}, \mathrm{C}=\mathrm{OB}\right)$, $167.8\left(\mathrm{~d},{ }^{2} J_{\mathrm{PC}}=18.9 \mathrm{~Hz}, \mathrm{C}=\mathrm{O}\right), 57.29\left(\mathrm{OCH}_{3}{ }^{\mathrm{B}}\right), 48.9\left(\mathrm{OCH}_{3}\right), 47.1\left(\mathrm{~d},{ }^{1} J_{\mathrm{PC}}=111.8 \mathrm{~Hz}\right.$, $\mathrm{C}=\mathrm{P})^{\mathrm{t}}, 42.39(\mathrm{CH}), 42.4\left(\mathrm{~d},{ }^{2} J_{\mathrm{PC}}=22.9 \mathrm{~Hz},>\mathrm{C}<\right)^{\mathrm{t}}, 38.3\left(\mathrm{~d},{ }^{1} J_{\mathrm{PC}}=35.3 \mathrm{~Hz},{ }^{t} \mathrm{Bu}^{\mathrm{a}}\right), 38.2$ $\left(\mathrm{d},{ }^{2} J_{\mathrm{PC}}=1.2 \mathrm{~Hz}, \mathrm{C}^{\mathrm{Me}}\right), 35.5\left(\mathrm{~d},{ }^{1} J_{\mathrm{PC}}=43.1 \mathrm{~Hz},{ }^{t} \mathrm{Bu}^{\mathrm{b}}\right), 32.2\left(\mathrm{~d},{ }^{1} J_{\mathrm{PC}}=53.7 \mathrm{~Hz}, \mathrm{PCH}_{2}\right)$, $28.5\left(\mathrm{~d},{ }^{2} J_{\mathrm{PC}}=0.9 \mathrm{~Hz},{ }^{t} \mathrm{Bu}^{\mathrm{b}}\right), 27.2\left(\mathrm{~d},{ }^{2} J_{\mathrm{PC}}=1.6 \mathrm{~Hz},{ }^{t} \mathrm{Bu}^{\mathrm{a}}\right), 16.1\left(\mathrm{~d},{ }^{3} J_{\mathrm{PC}}=6.2 \mathrm{~Hz}, \mathrm{CH}_{3}\right)$, 14.8 (br, $\left.\mathrm{BCH}_{2}\right),\left[\mathrm{C}_{6} \mathrm{~F}_{5}\right.$ not listed, ${ }^{\mathrm{t}}$ tentative assignment].

${ }^{11} \mathbf{B}\left\{{ }^{1} \mathrm{H}\right\}$ NMR $\left(160 \mathrm{MHz}, 299 \mathrm{~K}, \mathrm{CD}_{2} \mathrm{Cl}_{2}\right): \delta=3.8\left(v_{1 / 2} \sim 350 \mathrm{~Hz}\right)$.

${ }^{31} \mathbf{P}\left\{{ }^{1} \mathbf{H}\right\}$ NMR $\left(202 \mathrm{MHz}, 299 \mathrm{~K}, \mathrm{CD}_{2} \mathrm{Cl}_{2}\right): \delta=80.1\left(v_{1 / 2} \sim 1 \mathrm{~Hz}, 83 \mathrm{~mol} \%\right), 71.1\left(v_{1 / 2}\right.$ $\sim 0.9 \mathrm{~Hz}, 17 \mathrm{~mol} \%)$.

${ }^{19}$ F NMR $\left(470 \mathrm{MHz}, 299 \mathrm{~K}, \mathrm{CD}_{2} \mathrm{Cl}_{2}\right): \delta=$ major isomer $(84 \mathrm{~mol} \%)$ : $-135.1(\mathrm{~m}, 2 \mathrm{~F}, o)$, $-160.3\left(\mathrm{t},{ }^{3} J_{\mathrm{FF}}=20.1 \mathrm{~Hz}, 1 \mathrm{~F}, p\right),-166.1(\mathrm{~m}, 2 \mathrm{~F}, m)\left(\mathrm{C}_{6} \mathrm{~F}_{5}\right)\left[\Delta \delta^{19} \mathrm{~F}_{\mathrm{mp}}=5.8\right],-136.3(\mathrm{~m}, 2 \mathrm{~F}$, 
$o),-161.3\left(\mathrm{t},{ }^{3} J_{\mathrm{FF}}=20.1 \mathrm{~Hz}, 1 \mathrm{~F}, p\right),-165.5(\mathrm{~m}, 2 \mathrm{~F}, m)\left(\mathrm{C}_{6} \mathrm{~F}_{5}\right)\left[\Delta \delta^{19} \mathrm{~F}_{\mathrm{mp}}=4.2\right]$; minor isomer $16 \mathrm{~mol} \%)$ : $-135.6(\mathrm{~m}, 2 \mathrm{~F}, o),-161.6\left(\mathrm{t},{ }^{3} J_{\mathrm{FF}}=20.2 \mathrm{~Hz}, 1 \mathrm{~F}, p\right),-167.2(\mathrm{~m}, 2 \mathrm{~F}$, $m)\left(\mathrm{C}_{6} \mathrm{~F}_{5}\right)\left[\Delta \delta^{19} \mathrm{~F}_{\mathrm{mp}}=5.6\right],-136.1(\mathrm{~m}, 2 \mathrm{~F}, o),-161.4\left(\mathrm{t},{ }^{3} J_{\mathrm{FF}}=20.1 \mathrm{~Hz}, 1 \mathrm{~F}, p\right),-165.7(\mathrm{~m}$, $2 \mathrm{~F}, m)\left(\mathrm{C}_{6} \mathrm{~F}_{5}\right)\left[\Delta \delta^{19} \mathrm{~F}_{\mathrm{mp}}=4.3\right]$.

${ }^{1} \mathbf{H},{ }^{1} \mathrm{H}$-GCOSY $\left(500 \mathrm{MHz} / 500 \mathrm{MHz}, 299 \mathrm{~K}, \mathrm{CD}_{2} \mathrm{Cl}_{2}\right.$ )[selected traces]: $\delta{ }^{1} \mathrm{H} / \delta^{1} \mathrm{H}=$ major isomer: 2.34 / $2.18\left(\mathrm{PCH}_{2} / \mathrm{PCH}_{2}\right), 1.96$ / $0.87\left(\mathrm{BCH}_{2} / \mathrm{BCH}_{2}\right)$; minor isomer: $2.43 / 2.26\left(\mathrm{PCH}_{2} / \mathrm{PCH}_{2}\right), 1.88 / 0.84\left(\mathrm{BCH}_{2} / \mathrm{BCH}_{2}\right)$.

${ }^{1} \mathbf{H},{ }^{13} \mathbf{C}-G H S Q C\left(500 \mathrm{MHz} / 126 \mathrm{MHz}, 299 \mathrm{~K}, \mathrm{CD}_{2} \mathrm{Cl}_{2}\right): \delta{ }^{1} \mathrm{H} / \delta{ }^{13} \mathrm{C}=$ major isomer: $4.07 / 57.34\left(\mathrm{OCH}_{3}{ }^{\mathrm{B}}\right), 2.88 / 49.3\left(\mathrm{OCH}_{3}\right), 2.34,2.18 / 30.5\left(\mathrm{PCH}_{2}\right), 1.96 / 42.40(\mathrm{CH})$, 1.96, $0.87 / 14.7\left(\mathrm{BCH}_{2}\right), 1.40 / 27.4\left({ }^{t} \mathrm{Bu}^{\mathrm{a}}\right), 1.39 / 28.6\left({ }^{t} \mathrm{Bu}^{\mathrm{b}}\right), 1.26 / 16.4\left(\mathrm{CH}_{3}\right)$; minor isomer: $4.07 / 57.29\left(\mathrm{OCH}_{3}{ }^{\mathrm{B}}\right), 3.28 / 48.9\left(\mathrm{OCH}_{3}\right), 2.43,2.26 / 32.2\left(\mathrm{PCH}_{2}\right), 1.88 / 42.39$ $(\mathrm{CH}), 1.88,0.84 / 14.7\left(\mathrm{BCH}_{2}\right), 1.35 / 27.2\left({ }^{t} \mathrm{Bu}^{\mathrm{a}}\right), 1.31 / 28.5\left({ }^{t} \mathrm{Bu}^{\mathrm{b}}\right), 1.25 / 16.1\left(\mathrm{CH}_{3}\right)$.

${ }^{1} \mathbf{H},{ }^{13} \mathbf{C}$-GHMBC $\left(500 \mathrm{MHz} / 126 \mathrm{MHz}, 299 \mathrm{~K}, \mathrm{CD}_{2} \mathrm{Cl}_{2}\right)$ [selected traces]: $\delta{ }^{1} \mathrm{H} / \delta^{13} \mathrm{C}$ = major isomer: 4.07, $1.96 / 187.0\left(\mathrm{OCH}_{3}{ }^{\mathrm{B}}, \mathrm{CH} / \mathrm{C}=\mathrm{OB}\right), 2.88 / 168.2\left(\mathrm{OCH}_{3} / \mathrm{C}=\mathrm{O}\right)$, 2.34, 2.18, 1.26, 0.87 / $40.5\left(\mathrm{PCH}_{2}, \mathrm{PCH}_{2}, \mathrm{CH}_{3}, \mathrm{BCH}_{2} / \mathrm{C}{ }^{\mathrm{Me}}\right), 2.18,1.96$ / $48.4\left(\mathrm{PCH}_{2}\right.$, $\mathrm{CH} / \mathrm{C}=\mathrm{P}), 1.96,1.26,0.87 / 41.1\left(\mathrm{CH}, \mathrm{CH}_{3}, \mathrm{BCH}_{2} />\mathrm{C}<\right), 1.40 / 38.8\left({ }^{t} \mathrm{Bu}^{\mathrm{a}} /{ }^{t} \mathrm{Bu}^{\mathrm{a}}\right)$, $1.39 / 35.1\left({ }^{t} \mathrm{Bu}^{\mathrm{b}} /{ }^{t} \mathrm{Bu}^{\mathrm{b}}\right)$; minor isomer: $4.07 / 186.4\left(\mathrm{OCH}_{3}{ }^{\mathrm{B}} / \mathrm{C}=\mathrm{OB}\right), 3.28 / 167.8$ $\left(\mathrm{OCH}_{3} / \mathrm{C}=\mathrm{O}\right), 2.43,1.25,0.84$ / $38.2\left(\mathrm{PCH}_{2}, \mathrm{CH}_{3}, \mathrm{BCH}_{2} / \mathrm{C}^{\mathrm{Me}}\right), 1.35 / 38.3\left({ }^{t} \mathrm{Bu}^{\mathrm{a}} /\right.$ $\left.{ }^{t} \mathrm{Bu}^{\mathrm{a}}\right), 1.31 / 35.5\left({ }^{t} \mathrm{Bu}^{\mathrm{b}} /{ }^{t} \mathrm{Bu}^{\mathrm{b}}\right)$.

${ }^{19} \mathbf{F},{ }^{19} \mathrm{~F}-G C O S Y\left(470 \mathrm{MHz} / 470 \mathrm{MHz}, 299 \mathrm{~K}, \mathrm{CD}_{2} \mathrm{Cl}_{2}\right): \delta{ }^{19} \mathrm{~F} / \delta{ }^{19} \mathrm{~F}=$ major isomer: $-166.1 /-135.1,-160.3\left(m-\mathrm{C}_{6} \mathrm{~F}_{5}^{\mathrm{a}} / o-\mathrm{C}_{6} \mathrm{~F}_{5}^{\mathrm{a}}, p-\mathrm{C}_{6} \mathrm{~F}_{5}{ }^{\mathrm{a}}\right),-165.5 /-136.3,-161.3\left(m-\mathrm{C}_{6} \mathrm{~F}_{5}{ }^{\mathrm{b}} /\right.$ $\left.o-\mathrm{C}_{6} \mathrm{~F}_{5}{ }^{\mathrm{b}}, p-\mathrm{C}_{6} \mathrm{~F}_{5}{ }^{\mathrm{b}}\right)$; minor isomer: $-167.2 /-135.6,-161.6\left(m-\mathrm{C}_{6} \mathrm{~F}_{5}^{\mathrm{a}} / o-\mathrm{C}_{6} \mathrm{~F}_{5}^{\mathrm{a}}, p-\mathrm{C}_{6} \mathrm{~F}_{5}{ }^{\mathrm{a}}\right)$, $165.7 /-136.1,-161.4\left(m-\mathrm{C}_{6} \mathrm{~F}_{5} \mathrm{~b} / o-\mathrm{C}_{6} \mathrm{~F}_{5} \mathrm{~b}, p-\mathrm{C}_{6} \mathrm{~F}_{5}{ }^{\mathrm{b}}\right)$.

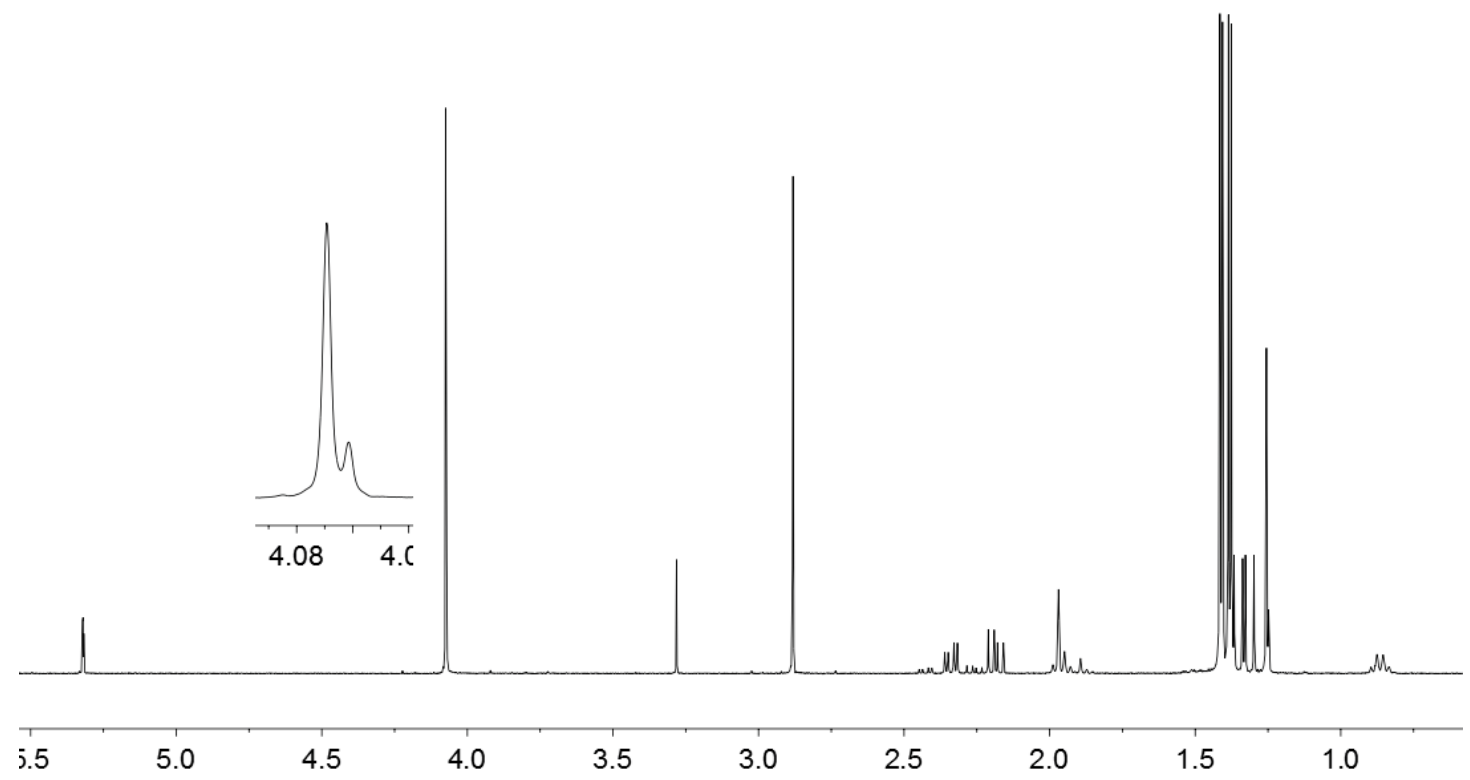

Figure S50. ${ }^{1} \mathrm{H}$ NMR $\left(500 \mathrm{MHz}, 299 \mathrm{~K}, \mathrm{CD}_{2} \mathrm{Cl}_{2}\right)$ of compound 12 

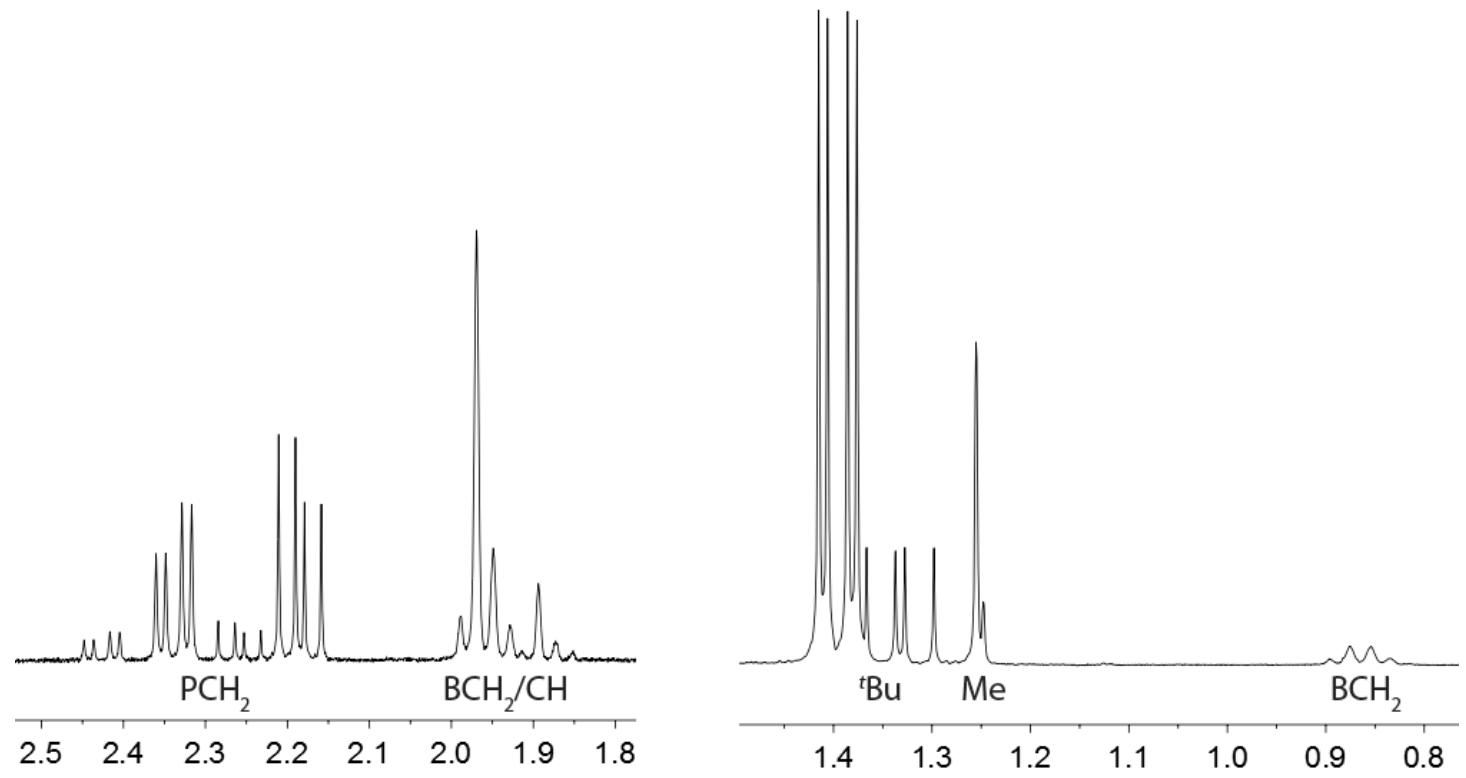

Figure S51. ${ }^{1} \mathrm{H}$ NMR $\left(500 \mathrm{MHz}, 299 \mathrm{~K}, \mathrm{CD}_{2} \mathrm{Cl}_{2}\right)$ of compound 12

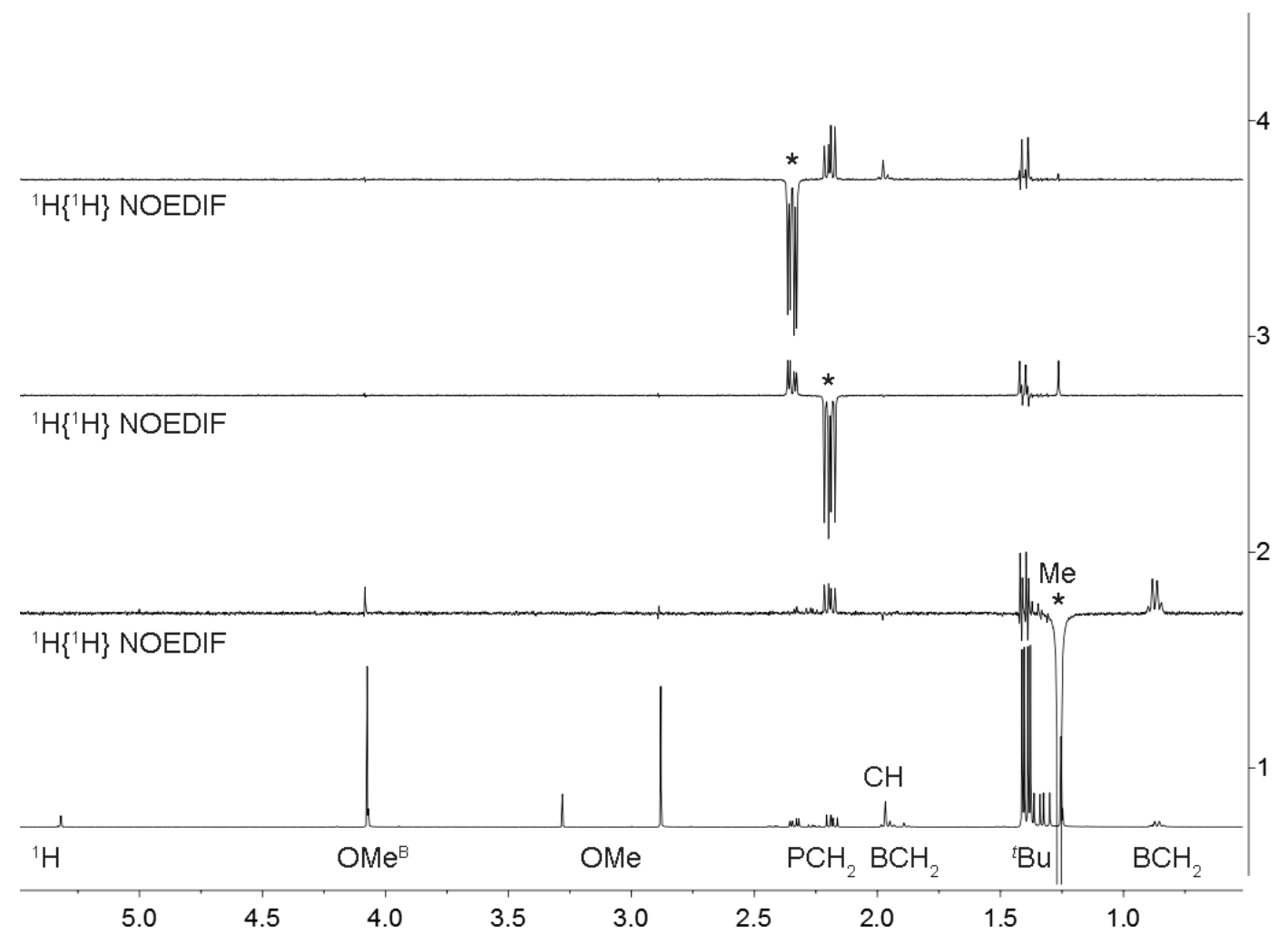

Figure S52. (1) ${ }^{\mathbf{1}} \mathbf{H}$ NMR (600 MHz, $\left.299 \mathrm{~K}, \mathrm{CD}_{2} \mathrm{Cl}_{2}\right)$ and (2) ${ }^{\mathbf{1}} \mathbf{H}\left\{{ }^{1} \mathbf{H}\right\}$ NOEDIF (600 $\mathrm{MHz}, 299 \mathrm{~K}, \mathrm{CD}_{2} \mathrm{Cl}_{2}$ ) spectra of compound 12 [* irradiation point] 


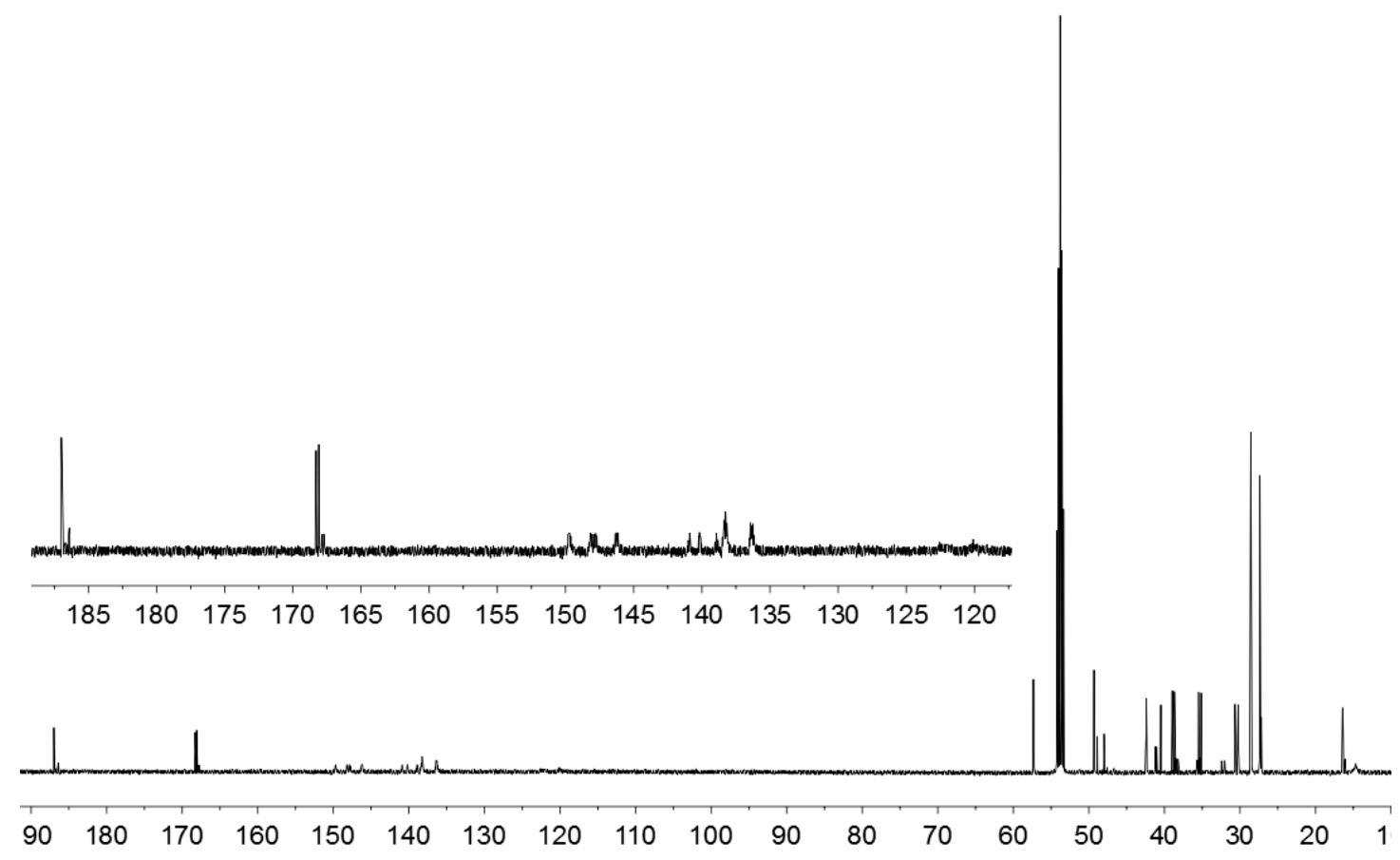

Figure S53. ${ }^{13} \mathbf{C}\left\{{ }^{1} \mathbf{H}\right\}$ NMR $\left(126 \mathrm{MHz}, 299 \mathrm{~K}, \mathrm{CD}_{2} \mathrm{Cl}_{2}\right)$ of compound 12

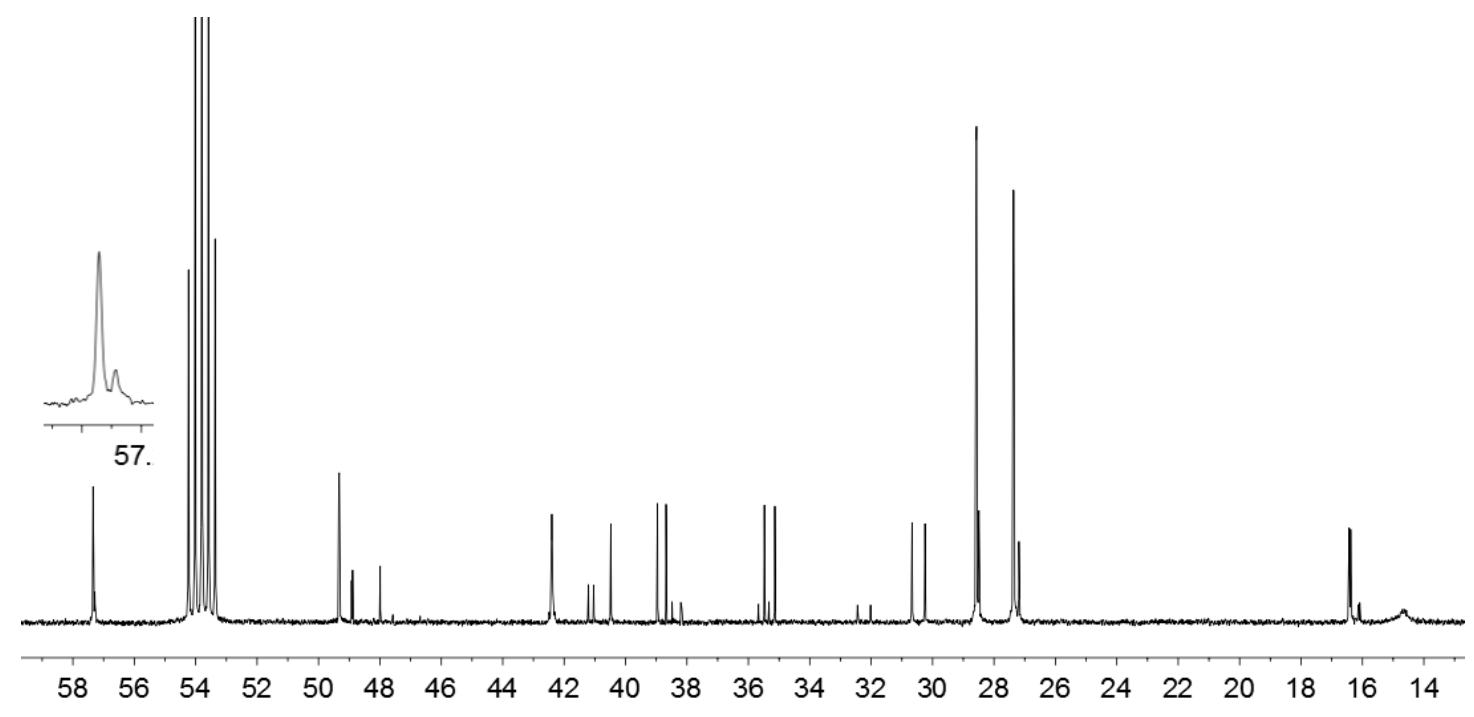

Figure S54. ${ }^{13} \mathbf{C}\left\{{ }^{1} \mathbf{H}\right\}$ NMR $\left(126 \mathrm{MHz}, 299 \mathrm{~K}, \mathrm{CD}_{2} \mathrm{Cl}_{2}\right)$ of compound $\mathbf{1 2}$ 


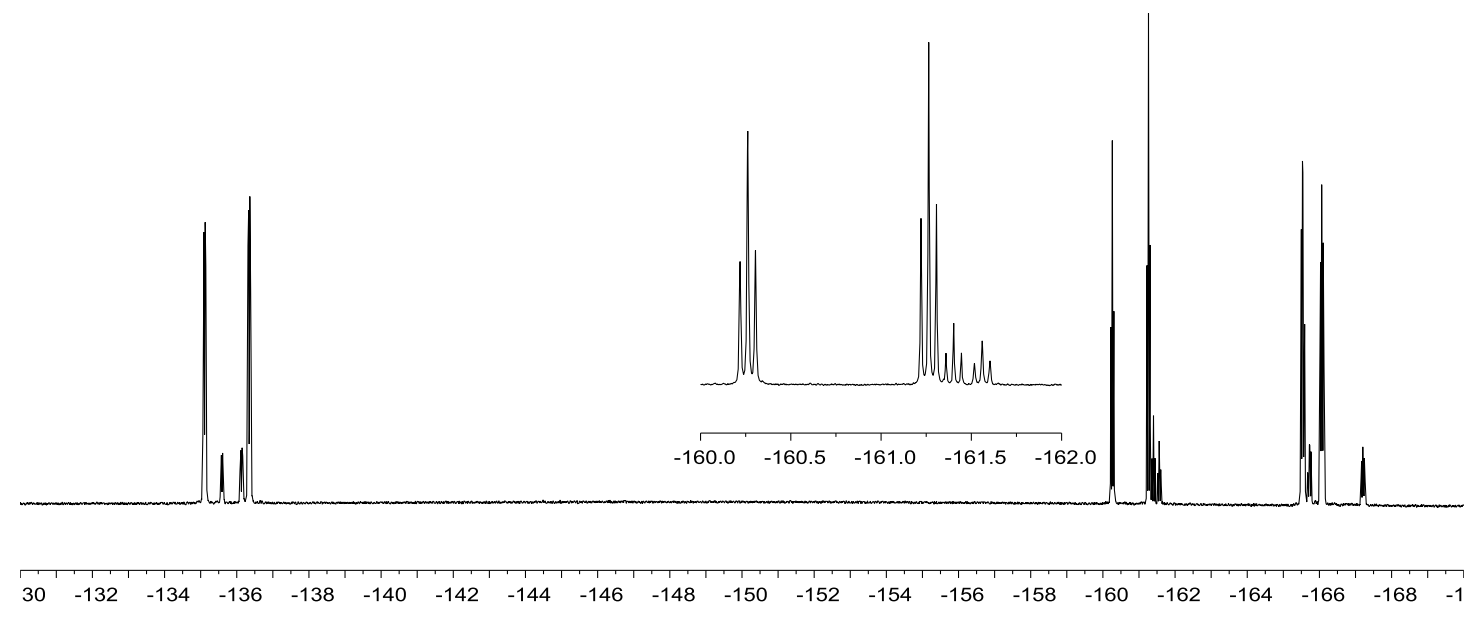

Figure S55. ${ }^{19} \mathbf{F}$ NMR $\left(470 \mathrm{MHz}, 299 \mathrm{~K}, \mathrm{CD}_{2} \mathrm{Cl}_{2}\right)$ of compound 12

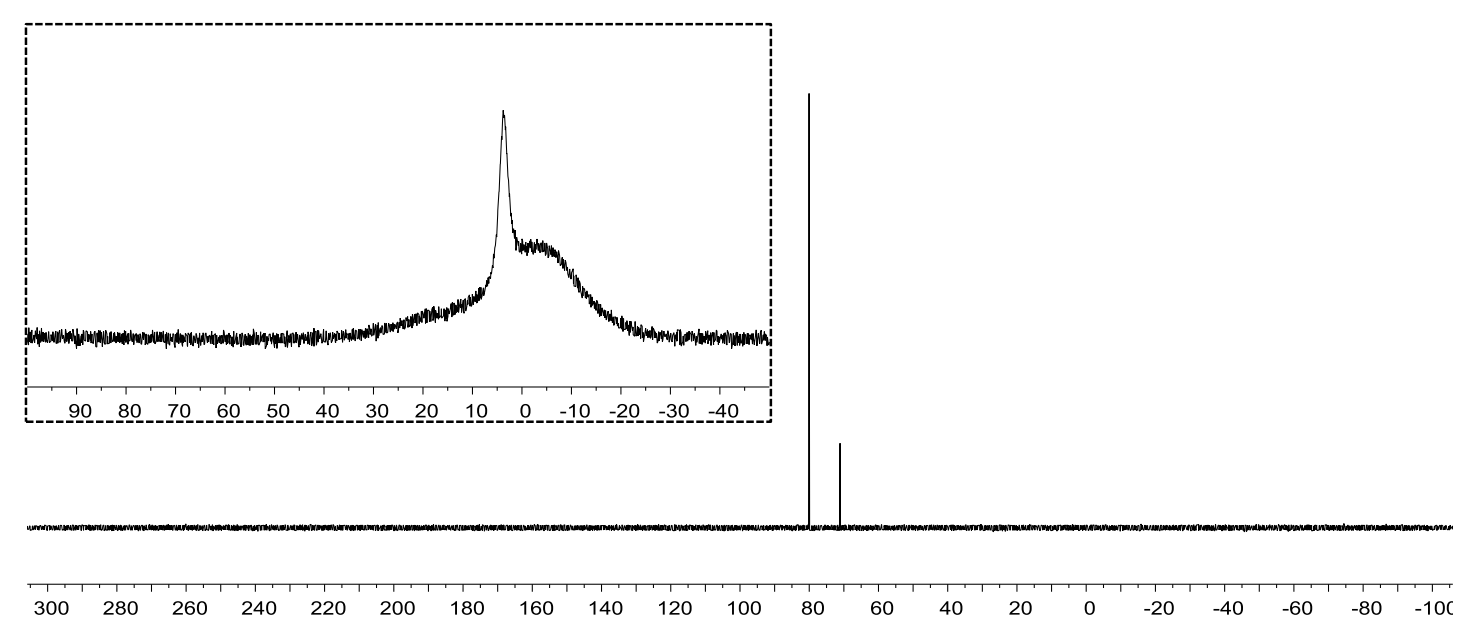

Figure S56. ${ }^{11} \mathbf{B}\left\{{ }^{1} \mathbf{H}\right\}$ NMR $\left(160 \mathrm{MHz}, 299 \mathrm{~K}, \mathrm{CD}_{2} \mathrm{Cl}_{2}\right)$ and ${ }^{31} \mathbf{P}\left\{{ }^{1} \mathbf{H}\right\}$ NMR $(202 \mathrm{MHz}$, $299 \mathrm{~K}, \mathrm{CD}_{2} \mathrm{Cl}_{2}$ ) of compound $\mathbf{1 2}$

X-ray crystal structure analysis of compound 12: formula $\mathrm{C}_{31} \mathrm{H}_{32} \mathrm{BF}_{10} \mathrm{O}_{4} \mathrm{P}, M=$ 700.35 , colourless crystal, $0.30 \times 0.23 \times 0.15 \mathrm{~mm}, a=12.1821(2), b=18.7258(3), c=$ 14.2778(2) $\AA, \beta=105.022(1)^{\circ}, V=3145.7(1) \AA^{3}, \rho_{\text {calc }}=1.479 \mathrm{gcm}^{-3}, \mu=0.183 \mathrm{~mm}^{-1}$, empirical absorption correction ( $0.947 \leq \mathrm{T} \leq 0.973), Z=4$, monoclinic, space group $P 2{ }_{1} / \mathrm{c}$ (No. 14), $\lambda=0.71073 \AA, T=223(2) \mathrm{K}, \omega$ and $\varphi$ scans, 19786 reflections collected $( \pm h, \pm k, \pm l), 5602$ independent $\left(R_{\text {int }}=0.033\right)$ and 4972 observed reflections $[I>2 \sigma(I)]$, 433 refined parameters, $R=0.048, w R^{2}=0.115$, max. (min.) residual electron density $0.49(-0.33)$ e. $\AA^{-3}$, hydrogen atoms were calculated and refined as riding atoms. 


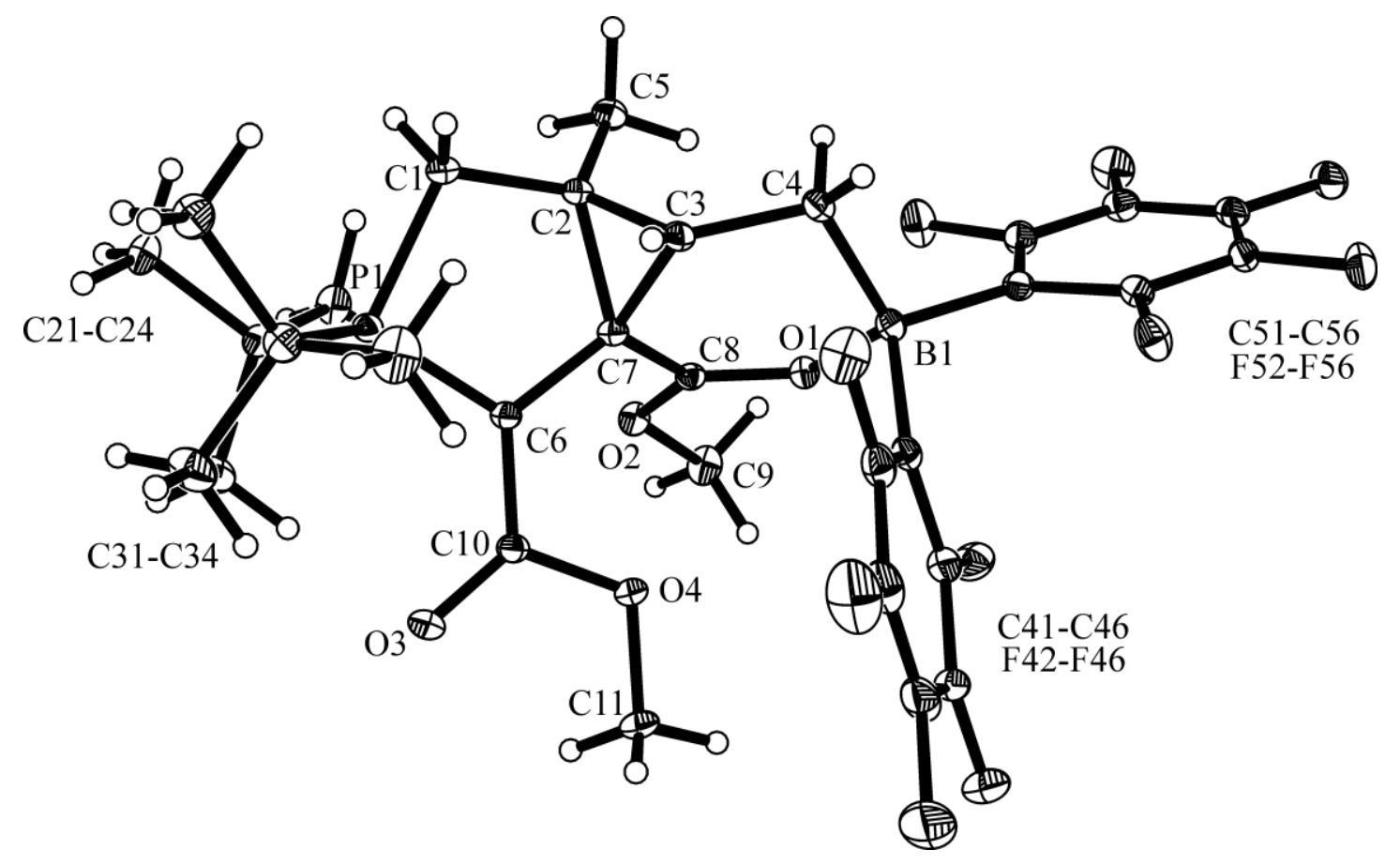

Figure S57. 


\section{Synthesis of compound 16}
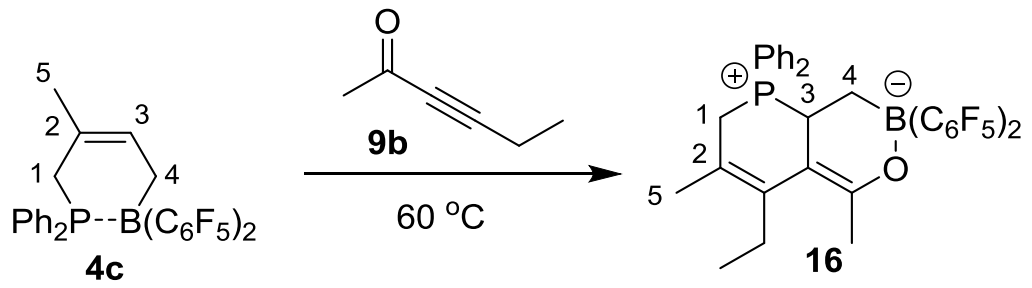

\section{Scheme S8}

A solution of compounds $\mathbf{4 c}(119.6 \mathrm{mg}, 0.2 \mathrm{mmol})$ and $\mathbf{9 b}(19.2 \mathrm{mg}, 0.2 \mathrm{mmol})$ in toluene $(2 \mathrm{~mL})$ was stirred at $60{ }^{\circ} \mathrm{C}$ for 3 days. Then all volatiles were removed in vacuo and the resulting residue was washed with cold pentane $(3 \times 1 \mathrm{~mL})$. After drying in vacuo compound 16 (101.5 mg, $73 \%$ ) was obtained as a light yellow solid. Crystals suitable for the X-ray crystal structure analysis were obtained by slow diffusion of pentane to a solution of compound 16 in dichloromethane at $-35{ }^{\circ}$ C. M.p. $195{ }^{\circ} \mathrm{C}$. Anal. Calc. for $\mathrm{C}_{35} \mathrm{H}_{26} \mathrm{BF}_{10} \mathrm{OP}: \mathrm{C}: 60.54 ; \mathrm{H}: 3.77$. Found: C: 60.75; H: 3.93 .

${ }^{1} \mathbf{H}$ NMR $\left(600 \mathrm{MHz}, 299 \mathrm{~K}, \mathrm{CD}_{2} \mathrm{Cl}_{2}\right): \delta=7.77,7.75$ (each m, each $\left.1 \mathrm{H}, p-\mathrm{Ph}\right), 7.62(\mathrm{~m}$, $6 \mathrm{H}, m, m, o-\mathrm{Ph}), 7.50(\mathrm{~m}, 2 \mathrm{H}, o-\mathrm{Ph}), 3.39(\mathrm{~m}, 1 \mathrm{H}, \mathrm{CH}), 3.35\left(\mathrm{ddm},{ }^{2} J_{\mathrm{HH}}=15.8 \mathrm{~Hz},{ }^{2} J_{\mathrm{PH}}\right.$ $\left.=14.8 \mathrm{~Hz}, 1 \mathrm{H}, \mathrm{PCH}_{2}\right), 2.79\left(\mathrm{dd},{ }^{2} J_{\mathrm{HH}}=15.8 \mathrm{~Hz},{ }^{2} J_{\mathrm{PH}}=12.1 \mathrm{~Hz}, 1 \mathrm{H}, \mathrm{PCH}_{2}\right), 2.45(\mathrm{~m})$, 2.18 (br m) (each 1H, Et), $1.79\left(\mathrm{dd},{ }^{5} \mathrm{~J}_{\mathrm{PH}}=5.6 \mathrm{~Hz},{ }^{a} J_{\mathrm{HH}}=1.6 \mathrm{~Hz},{ }^{\mathrm{a}} 3 \mathrm{H},{ }^{\mathrm{O}-\mathrm{C}} \mathrm{CH}_{3}\right), 1.59$ $\left(\mathrm{m}, 3 \mathrm{H}, \mathrm{CH}_{3}\right), 1.45,0.83$ (each m, each $\left.1 \mathrm{H}, \mathrm{BCH}_{2}\right), 0.72\left(\mathrm{t},{ }^{3} J_{\mathrm{HH}}=7.4 \mathrm{~Hz}, 3 \mathrm{H}, \mathrm{Et}\right),{ }^{\mathrm{a}}$ assignment by ${ }^{1} \mathrm{H}\left\{{ }^{1} \mathrm{H}\right\}$ and ${ }^{1} \mathrm{H}\left\{{ }^{31} \mathrm{P}\right\}$ NMR experiments].

${ }^{13} \mathbf{C}\left\{{ }^{1} \mathbf{H}\right\}$ NMR $\left(151 \mathrm{MHz}, 299 \mathrm{~K}, \mathrm{CD}_{2} \mathrm{Cl}_{2}\right): \delta=159.3\left(\mathrm{~d},{ }^{3} J_{\mathrm{PC}}=11.5 \mathrm{~Hz},=\mathrm{CO}\right), 145.0$ $\left(\mathrm{d},{ }^{3} J_{\mathrm{PC}}=12.0 \mathrm{~Hz},=\mathrm{CEt}\right), 134.59,134.55\left(\right.$ each d, $\left.{ }^{4} J_{\mathrm{PC}}=2.9 \mathrm{~Hz}, p-\mathrm{Ph}\right), 133.8,133.3$ $\left(\right.$ each d, $\left.{ }^{2} J_{\mathrm{PC}}=8.0 \mathrm{~Hz}, o-\mathrm{Ph}\right), 130.2\left(\mathrm{~d},{ }^{3} J_{\mathrm{PC}}=11.1 \mathrm{~Hz}\right), 129.7\left(\mathrm{~d},{ }^{3} J_{\mathrm{PC}}=11.6 \mathrm{~Hz}\right)(\mathrm{m}-$ $\mathrm{Ph}), 121.5\left(\mathrm{~d},{ }^{1} J_{\mathrm{PC}}=30.9 \mathrm{~Hz}\right), 121.0\left(\mathrm{~d},{ }^{1} J_{\mathrm{PC}}=32.9 \mathrm{~Hz}\right)(i-\mathrm{Ph}), 117.6\left(\mathrm{~d},{ }^{2} J_{\mathrm{PC}}=11.1 \mathrm{~Hz}\right.$, $=\mathrm{CMe}), 97.9\left(\mathrm{br} \mathrm{d},{ }^{2} J_{\mathrm{PC}}=11.2 \mathrm{~Hz},=\mathrm{C}\right), 33.0\left(\mathrm{~d},{ }^{1} J_{\mathrm{PC}}=45.8 \mathrm{~Hz}, \mathrm{CH}\right), 28.1\left(\mathrm{~d},{ }^{1} J_{\mathrm{PC}}=\right.$ $\left.49.5 \mathrm{~Hz}, \mathrm{PCH}_{2}\right), 27.6\left(\mathrm{~d},{ }^{4} \mathrm{~J}_{\mathrm{PC}}=4.0 \mathrm{~Hz}, \mathrm{Et}\right), 22.8\left(\mathrm{~d},{ }^{4} \mathrm{~J}_{\mathrm{PC}}=2.9 \mathrm{~Hz},{ }^{\mathrm{O}-\mathrm{C}_{\mathrm{CH}}}\right), 20.2(\mathrm{br}$, $\left.\mathrm{BCH}_{2}\right), 20.1\left(\mathrm{~d},{ }^{3} J_{\mathrm{PC}}=6.8 \mathrm{~Hz}, \mathrm{CH}_{3}\right), 12.9\left(\mathrm{~d},{ }^{5} J_{\mathrm{PC}}=3.1 \mathrm{~Hz}, \mathrm{Et}\right),\left[\mathrm{C}_{6} \mathrm{~F}_{5}\right.$ not listed $]$.

${ }^{11} \mathbf{B}\left\{{ }^{1} \mathbf{H}\right\}$ NMR $\left(192 \mathrm{MHz}, 299 \mathrm{~K}, \mathrm{CD}_{2} \mathrm{Cl}_{2}\right): \delta=-3.1\left(v_{1 / 2} \sim 90 \mathrm{~Hz}\right)$.

${ }^{10} \mathbf{B}\left\{{ }^{1} \mathbf{H}\right\}$ NMR $\left(54 \mathrm{MHz}, 299 \mathrm{~K}, \mathrm{CD}_{2} \mathrm{Cl}_{2}\right): \delta=-3.1\left(v_{1 / 2} \sim 70 \mathrm{~Hz}\right)$.

${ }^{31} \mathbf{P}\left\{{ }^{1} \mathrm{H}\right\}$ NMR $\left(243 \mathrm{MHz}, 299 \mathrm{~K}, \mathrm{CD}_{2} \mathrm{Cl}_{2}\right): \delta=27.3\left(v_{1 / 2} \sim 15 \mathrm{~Hz}\right)$.

${ }^{19}$ F NMR $\left(564 \mathrm{MHz}, 299 \mathrm{~K}, \mathrm{CD}_{2} \mathrm{Cl}_{2}\right): \delta=-134.5(\mathrm{~m}, 2 \mathrm{~F}, o),-162.5\left(\mathrm{t},{ }^{3} J_{\mathrm{FF}}=20.2 \mathrm{~Hz}\right.$, $1 \mathrm{~F}, p),-166.6(\mathrm{~m}, 2 \mathrm{~F}, m)\left(\mathrm{C}_{6} \mathrm{~F}_{5}\right)\left[\Delta \delta^{19} \mathrm{~F}_{\mathrm{mp}}=4.1\right],-136.6(\mathrm{~m}, 2 \mathrm{~F}, o),-162.9\left(\mathrm{t},{ }^{3} J_{\mathrm{FF}}=20.2\right.$ $\mathrm{Hz}, 1 \mathrm{~F}, p),-166.5(\mathrm{~m}, 2 \mathrm{~F}, m)\left(\mathrm{C}_{6} \mathrm{~F}_{5}\right)\left[\Delta \delta^{19} \mathrm{~F}_{\mathrm{mp}}=3.6\right]$.

${ }^{1} \mathbf{H},{ }^{1} \mathrm{H}-$ GCOSY $\left(600 \mathrm{MHz} / 600 \mathrm{MHz}, 299 \mathrm{~K}, \mathrm{CD}_{2} \mathrm{Cl}_{2}\right)$ [selected traces]: $\delta{ }^{1} \mathrm{H} / \delta^{1} \mathrm{H}=$ 3.39 / 1.45, $0.83\left(\mathrm{CH} / \mathrm{BCH}_{2}, \mathrm{BCH}_{2}\right), 3.35$ / 2.79, $1.59\left(\mathrm{PCH}_{2} / \mathrm{PCH}_{2}, \mathrm{CH}_{3}\right), 2.45$ / 2.18, $0.72(\mathrm{Et} / \mathrm{Et}, \mathrm{Et})$.

${ }^{1} \mathbf{H},{ }^{13} \mathbf{C}-G H S Q C\left(600 \mathrm{MHz} / 151 \mathrm{MHz}, 299 \mathrm{~K}, \mathrm{CD}_{2} \mathrm{Cl}_{2}\right): \delta{ }^{1} \mathrm{H} / \delta{ }^{13} \mathrm{C}=7.77 / 134.55$ $\left(p-\mathrm{Ph}^{\mathrm{a}}\right), 7.75 / 134.59\left(p-\mathrm{Ph}^{\mathrm{b}}\right), 7.62 / 130.2\left(m-\mathrm{Ph}^{\mathrm{b}}\right), 7.61 / 129.7\left(m-\mathrm{Ph}^{\mathrm{a}}\right), 7.61 / 133.3$ 
$\left(o-\mathrm{Ph}^{\mathrm{b}}\right), 7.50 / 133.8\left(o-\mathrm{Ph}^{\mathrm{a}}\right), 3.39 / 33.0(\mathrm{CH}), 3.35,2.79 / 28.1\left(\mathrm{PCH}_{2}\right), 2.45,2.18$ / 27.6 (Et), 1.79 / $22.8\left({ }^{\mathrm{O}-\mathrm{C}} \mathrm{CH}_{3}\right), 1.59 / 20.1\left(\mathrm{CH}_{3}\right), 1.45,0.83$ / $20.2\left(\mathrm{BCH}_{2}\right), 0.72 / 12.9$ (Et).

${ }^{\mathbf{1}} \mathbf{H},{ }^{13} \mathbf{C}-\mathbf{G H M B C}\left(600 \mathrm{MHz} / 151 \mathrm{MHz}, 299 \mathrm{~K}, \mathrm{CD}_{2} \mathrm{Cl}_{2}\right)$ [selected traces]: $\delta{ }^{1} \mathrm{H} / \delta{ }^{13} \mathrm{C}$ $=7.62 / 130.2,121.5\left(m-\mathrm{Ph}^{\mathrm{b}} / m-\mathrm{Ph}^{\mathrm{b}}, i-\mathrm{Ph}^{\mathrm{b}}\right), 7.61 / 129.7,121.0\left(m-\mathrm{Ph}^{\mathrm{a}} / m-\mathrm{Ph}^{\mathrm{a}}, i-\mathrm{Ph}^{\mathrm{a}}\right)$, 3.39, 1.79, 1.59 / $159.3\left(\mathrm{CH},{ }^{\mathrm{O}-\mathrm{C}_{\mathrm{CH}}}, \mathrm{CH}_{3} /=\mathrm{CO}\right), 3.39,2.45,2.18,1.79,1.59,1.45$ / $97.9\left(\mathrm{CH}, \mathrm{Et}, \mathrm{Et},{ }^{\mathrm{O}-\mathrm{C}} \mathrm{CH}_{3}, \mathrm{CH}_{3}, \mathrm{BCH}_{2} /=\mathrm{C}\right), 3.35,2.79,2.45,2.18,1.59,0.72 / 145.0$ $\left(\mathrm{PCH}_{2}, \mathrm{PCH}_{2}, \mathrm{Et}, \mathrm{Et}, \mathrm{CH}_{3}, \mathrm{Et} /=\mathrm{CEt}\right), 3.35,2.79,2.45,2.18,1.79,1.59 / 117.6\left(\mathrm{PCH}_{2}\right.$, $\left.\mathrm{PCH}_{2}, \mathrm{Et}, \mathrm{Et},{ }^{\mathrm{O}-\mathrm{C}} \mathrm{CH}_{3}, \mathrm{CH}_{3} /=\mathrm{CMe}\right)$.

${ }^{19} \mathbf{F},{ }^{19} \mathbf{F}-\mathbf{G C O S Y}\left(564 \mathrm{MHz} / 564 \mathrm{MHz}, 299 \mathrm{~K}, \mathrm{CD}_{2} \mathrm{Cl}_{2}\right): \delta{ }^{19} \mathrm{~F} / \delta{ }^{19} \mathrm{~F}=-166.6 /-134.5$, $-162.5\left(m-\mathrm{C}_{6} \mathrm{~F}_{5}^{\mathrm{a}} / o-\mathrm{C}_{6} \mathrm{~F}_{5}^{\mathrm{a}}, p-\mathrm{C}_{6} \mathrm{~F}_{5}^{\mathrm{a}}\right),-166.5 /-136.6,-162.9\left(m-\mathrm{C}_{6} \mathrm{~F}_{5} \mathrm{~b} / o-\mathrm{C}_{6} \mathrm{~F}_{5}^{\mathrm{b}}, p-\mathrm{C}_{6} \mathrm{~F}_{5}{ }^{\mathrm{b}}\right)$.

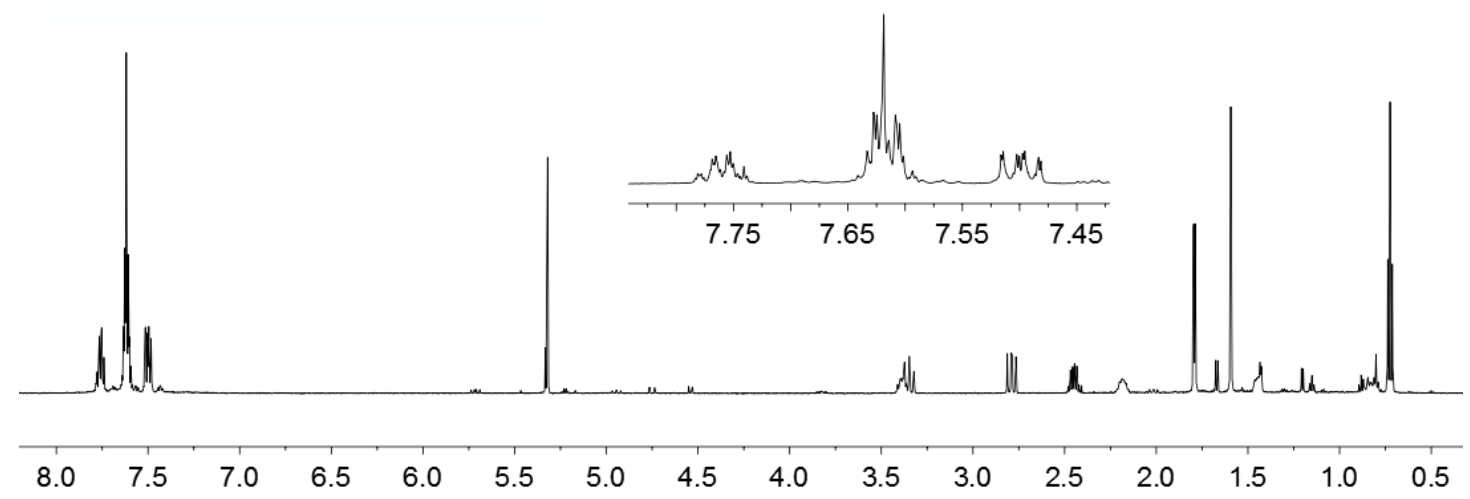

Figure S58. ${ }^{1} \mathrm{H}$ NMR $\left(600 \mathrm{MHz}, 299 \mathrm{~K}, \mathrm{CD}_{2} \mathrm{Cl}_{2}\right)$ of compound 16

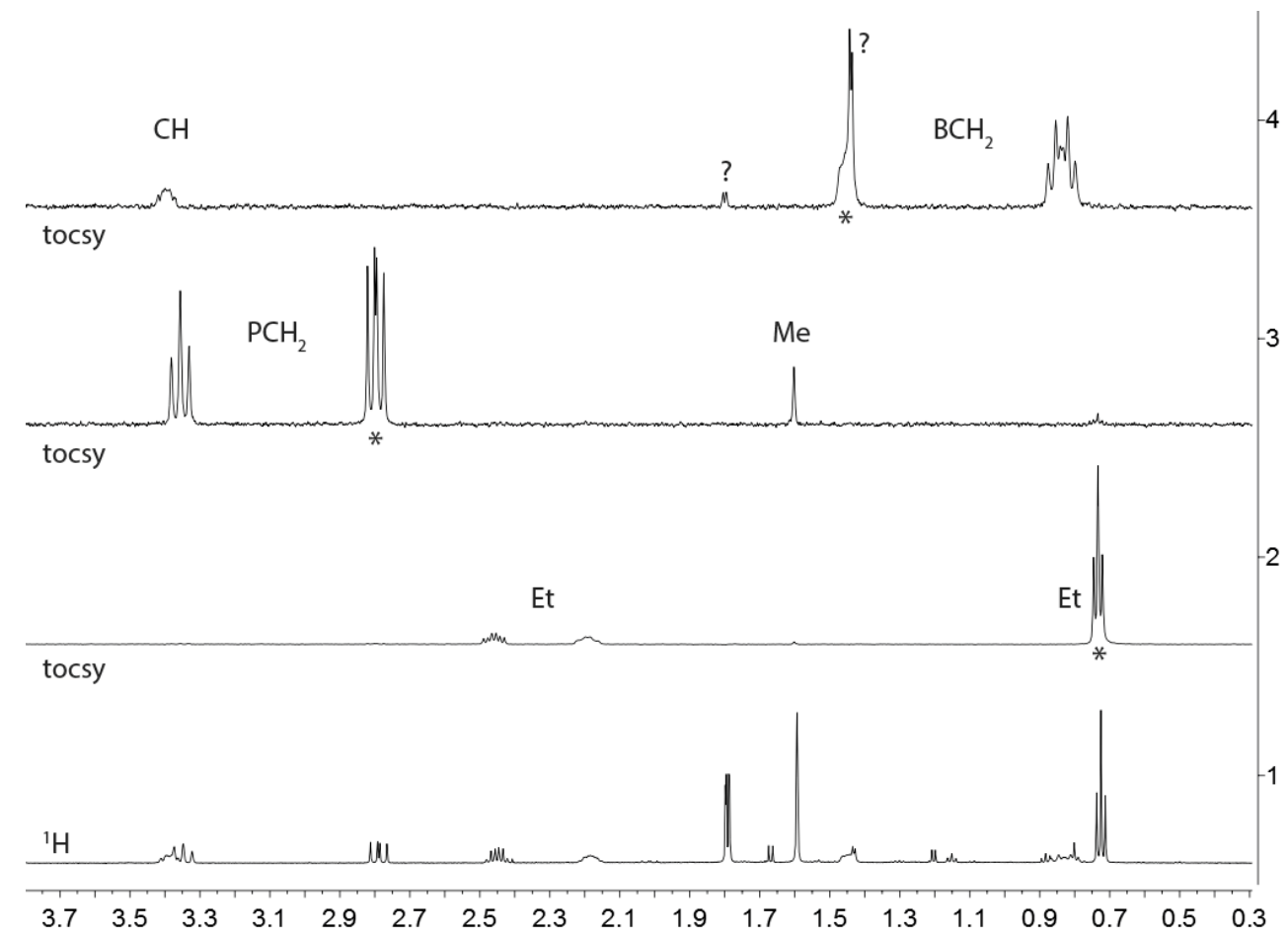

Figure S59. (1) ${ }^{\mathbf{1}} \mathbf{H}$ NMR (600 MHz, $299 \mathrm{~K}, \mathrm{CD}_{2} \mathrm{Cl}_{2}$ ) and (2 to 4) ${ }^{\mathbf{1}} \mathbf{H},{ }^{\mathbf{1}} \mathbf{H}$-TOCSY (600 $\mathrm{MHz} / 600 \mathrm{MHz}, 299 \mathrm{~K}, \mathrm{CD}_{2} \mathrm{Cl}_{2}$ ) of compound 16. Irradiation (*) at (2) $\delta^{1} \mathrm{H}_{\mathrm{irr}}=0.72$ (Et), (3) $\delta^{1} \mathrm{H}_{\text {irr }}=2.79\left(\mathrm{PCH}_{2}\right)$, and (4) $\delta^{1} \mathrm{H}_{\text {irr }}=1.45\left(\mathrm{BCH}_{2}\right)$. 

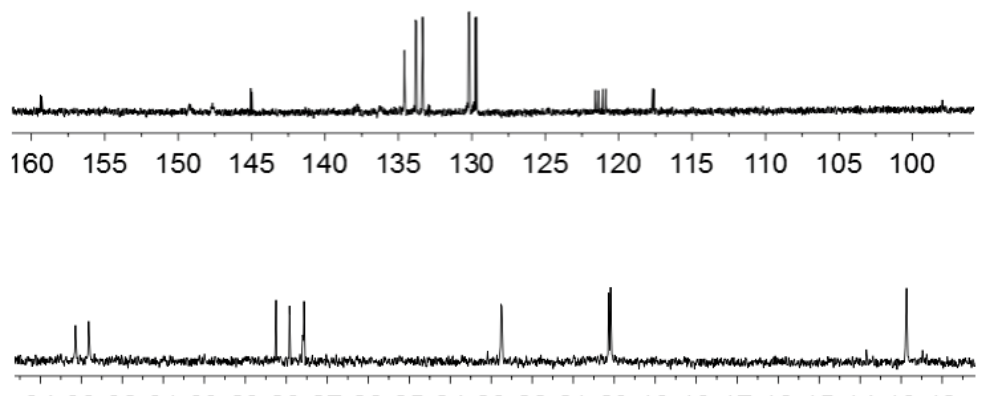

3433323130292827262524232221201918171615141312

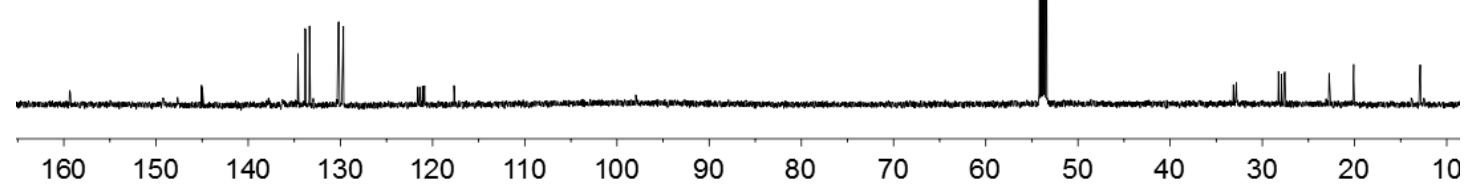

Figure S60. ${ }^{13} \mathbf{C}\left\{{ }^{1} \mathbf{H}\right\}$ NMR $\left(151 \mathrm{MHz}, 299 \mathrm{~K}, \mathrm{CD}_{2} \mathrm{Cl}_{2}\right)$ of compound $\mathbf{1 6}$

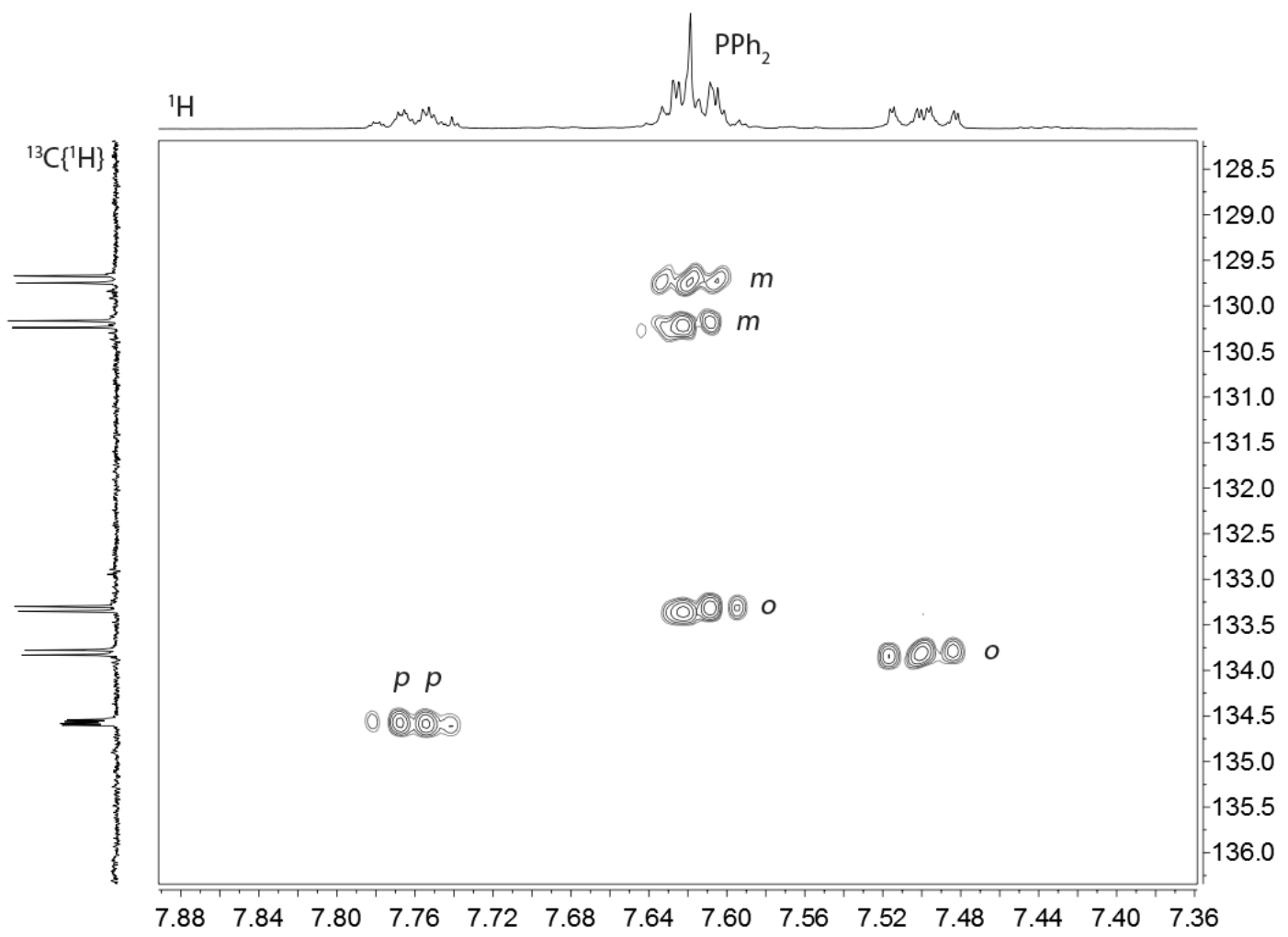

Figure S61. ${ }^{1} \mathbf{H},{ }^{13} \mathbf{C}-G H S Q C\left(600 \mathrm{MHz} / 151 \mathrm{MHz}, 299 \mathrm{~K}, \mathrm{CD}_{2} \mathrm{Cl}_{2}\right)$ of compound $\mathbf{1 6}$ 


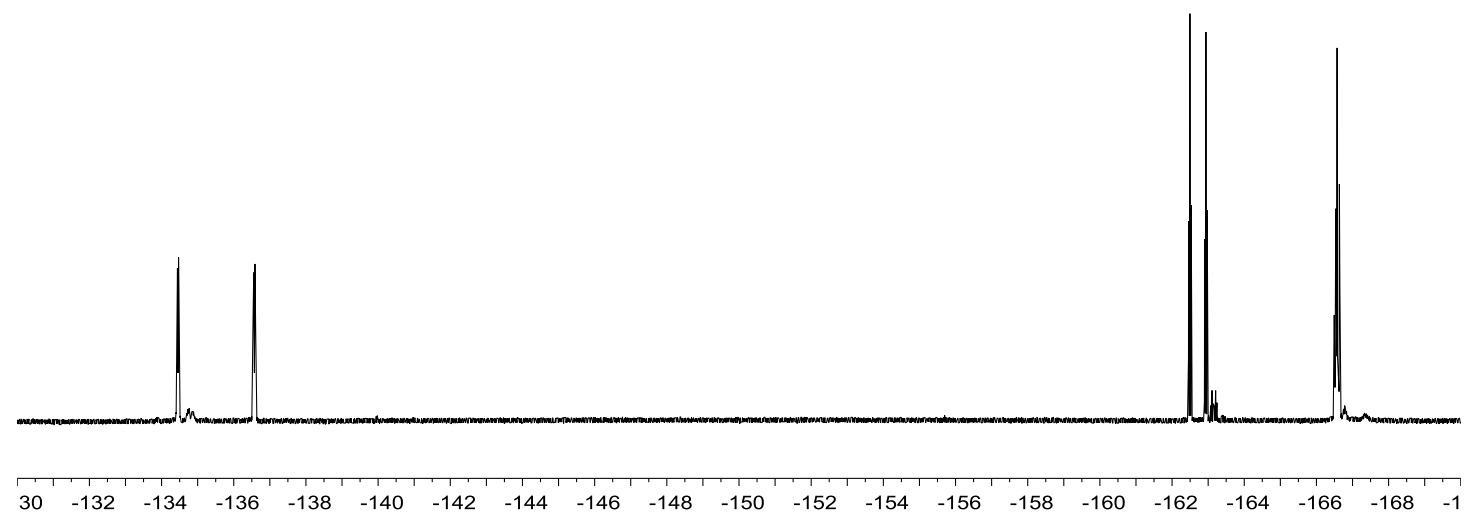

Figure S62. ${ }^{19} \mathbf{F}$ NMR $\left(564 \mathrm{MHz}, 299 \mathrm{~K}, \mathrm{CD}_{2} \mathrm{Cl}_{2}\right)$ of compound 16

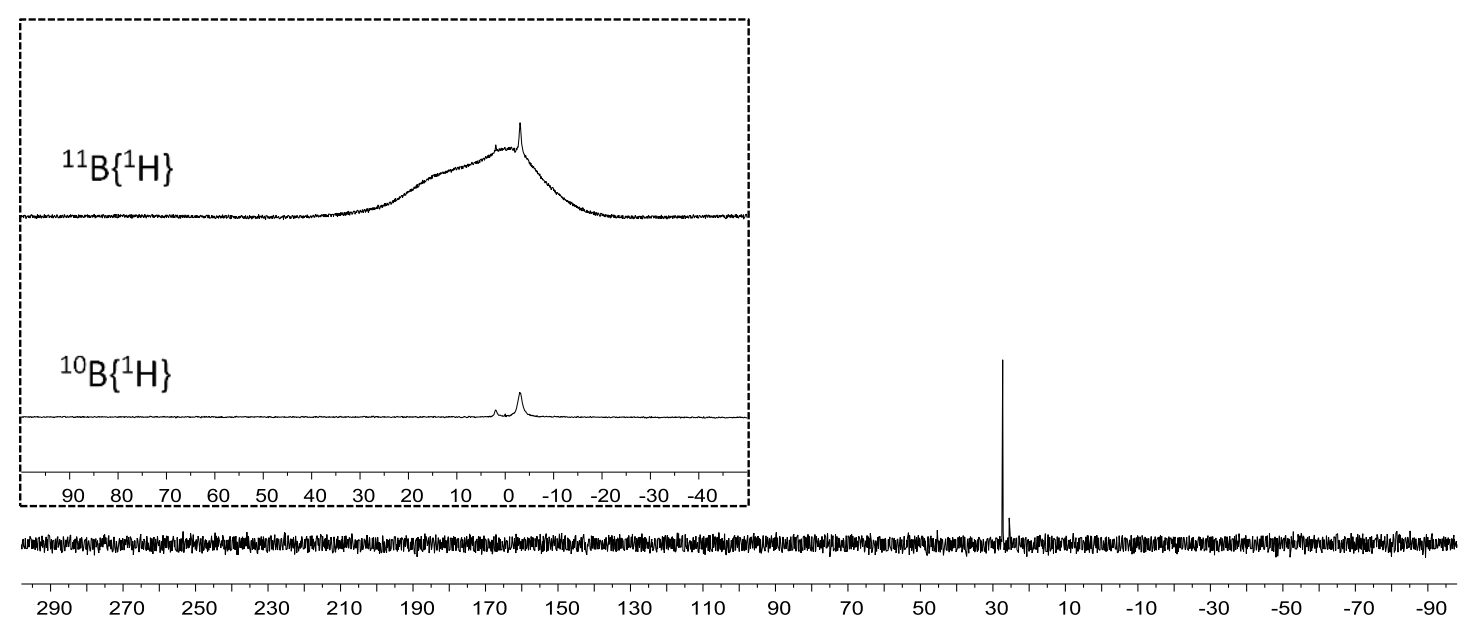

Figure S63. ${ }^{11} \mathbf{B}\left\{{ }^{1} \mathbf{H}\right\}$ NMR $\left(192 \mathrm{MHz}, 299 \mathrm{~K}, \mathrm{CD}_{2} \mathrm{Cl}_{2}\right),{ }^{\mathbf{1 0}} \mathbf{B}\left\{{ }^{\mathbf{1}} \mathbf{H}\right\}$ NMR $(54 \mathrm{MHz}, 299$ $\left.\mathrm{K}, \mathrm{CD}_{2} \mathrm{Cl}_{2}\right)$ and ${ }^{31} \mathbf{P}\left\{{ }^{1} \mathbf{H}\right\} \mathbf{N M R}\left(243 \mathrm{MHz}, 299 \mathrm{~K}, \mathrm{CD}_{2} \mathrm{Cl}_{2}\right)$ of compound $\mathbf{1 6}$

X-ray crystal structure analysis of compound 16: formula $\mathrm{C}_{35} \mathrm{H}_{26} \mathrm{BF}_{10} \mathrm{OP}, M=$ 694.34, colourless crystal, $0.32 \times 0.15 \times 0.05 \mathrm{~mm}, a=10.3933(2), b=11.7676(2), c=$ 14.8634(4) $\AA, \alpha=104.124(1), \beta=101.199(1), \gamma=111.497(1)^{\circ}, V=1557.1(1) \AA^{3}, \rho_{\text {calc }}$ $=1.481 \mathrm{gcm}^{-3}, \mu=0.178 \mathrm{~mm}^{-1}$, empirical absorption correction $(0.945 \leq \mathrm{T} \leq 0.991), Z$ $=2$, triclinic, space group $P^{\overline{1}}$ (No. 2$), \lambda=0.71073 \AA, T=223(2) \mathrm{K}, \omega$ and $\varphi$ scans, 14326 reflections collected $( \pm h, \pm k, \pm l), 5251$ independent $\left(R_{\text {int }}=0.057\right)$ and 4479 observed reflections $[I>2 \sigma(I)], 436$ refined parameters, $R=0.057, w R^{2}=0.121$, $\max$. (min.) residual electron density $0.30(-0.26) \mathrm{e} . \AA^{-3}$, hydrogen atoms were calculated and refined as riding atoms. 


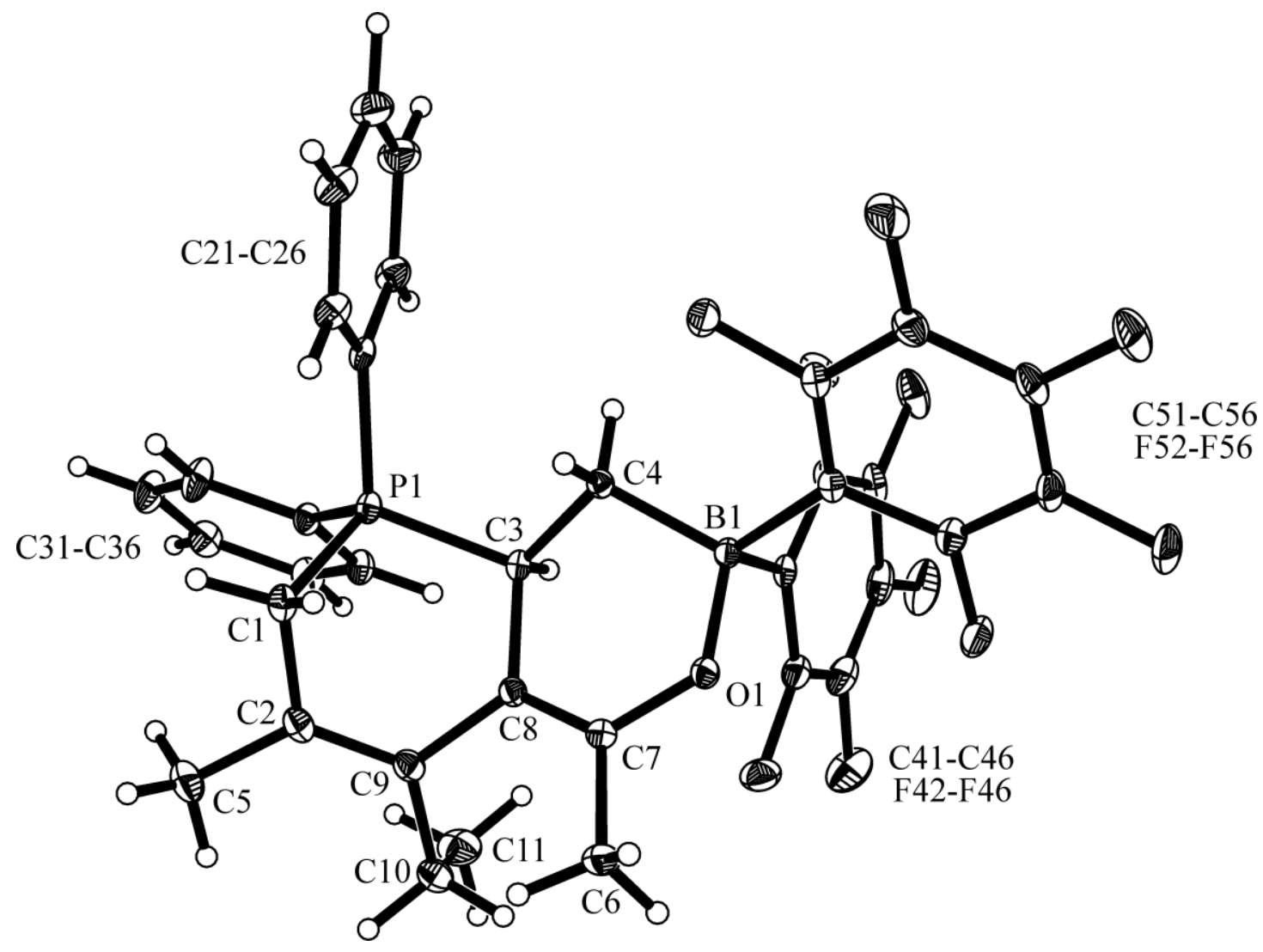

Figure S64. 


\section{Control experiments $(4 c+9 b$ : NMR scale $)$}

Reactions of compounds $\mathbf{4 c}$ with alkyne $\mathbf{9 b}$ monitored by NMR spectroscopy

Alkyne $9 \mathbf{b}(0.03 \mathrm{mmol}, 2.9 \mathrm{mg})$ was added to a solution of compound $4 \mathbf{c}(0.03 \mathrm{mmol}$, $17.9 \mathrm{mg})$ in $\mathrm{C}_{6} \mathrm{D}_{6}(0.5 \mathrm{~mL})$. The reaction mixture was transferred to a NMR tube and monitored by NMR spectroscopy.

Comment: NMR spectra showed that there is no substantial reaction of compound $\mathbf{4 c}$ with the alkyne $\mathbf{9 b}$ at r.t. after 40 hours.

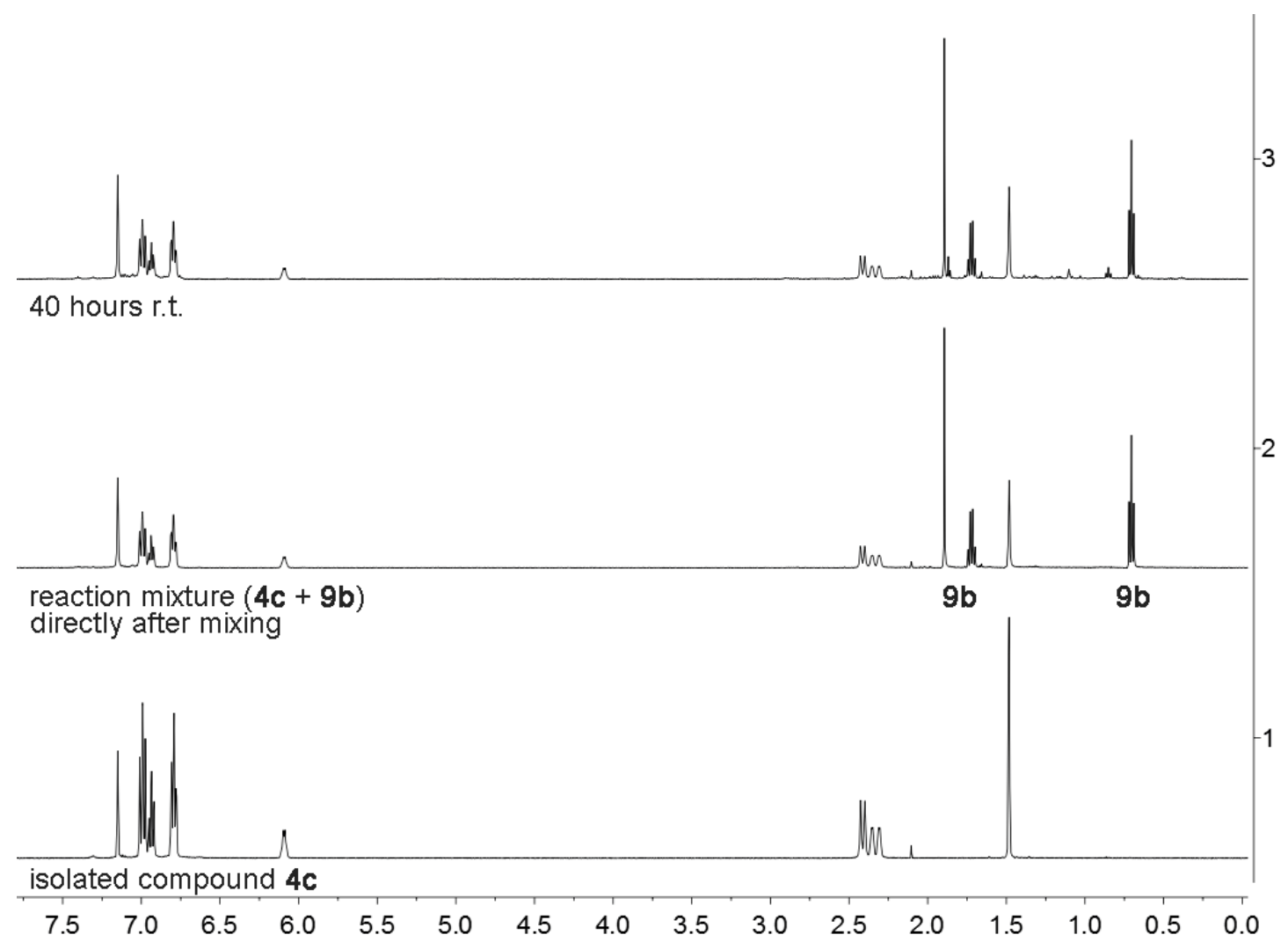

Figure S65. ${ }^{1} \mathbf{H}$ NMR $\left(500 \mathrm{MHz}, 299 \mathrm{~K}, \mathrm{C}_{6} \mathrm{D}_{6}\right)$ spectra of (1) isolated compound 4c and $(2,3)$ the reaction mixture of the reaction of compounds $\mathbf{4 c}$ with $\mathbf{9 b}$ 


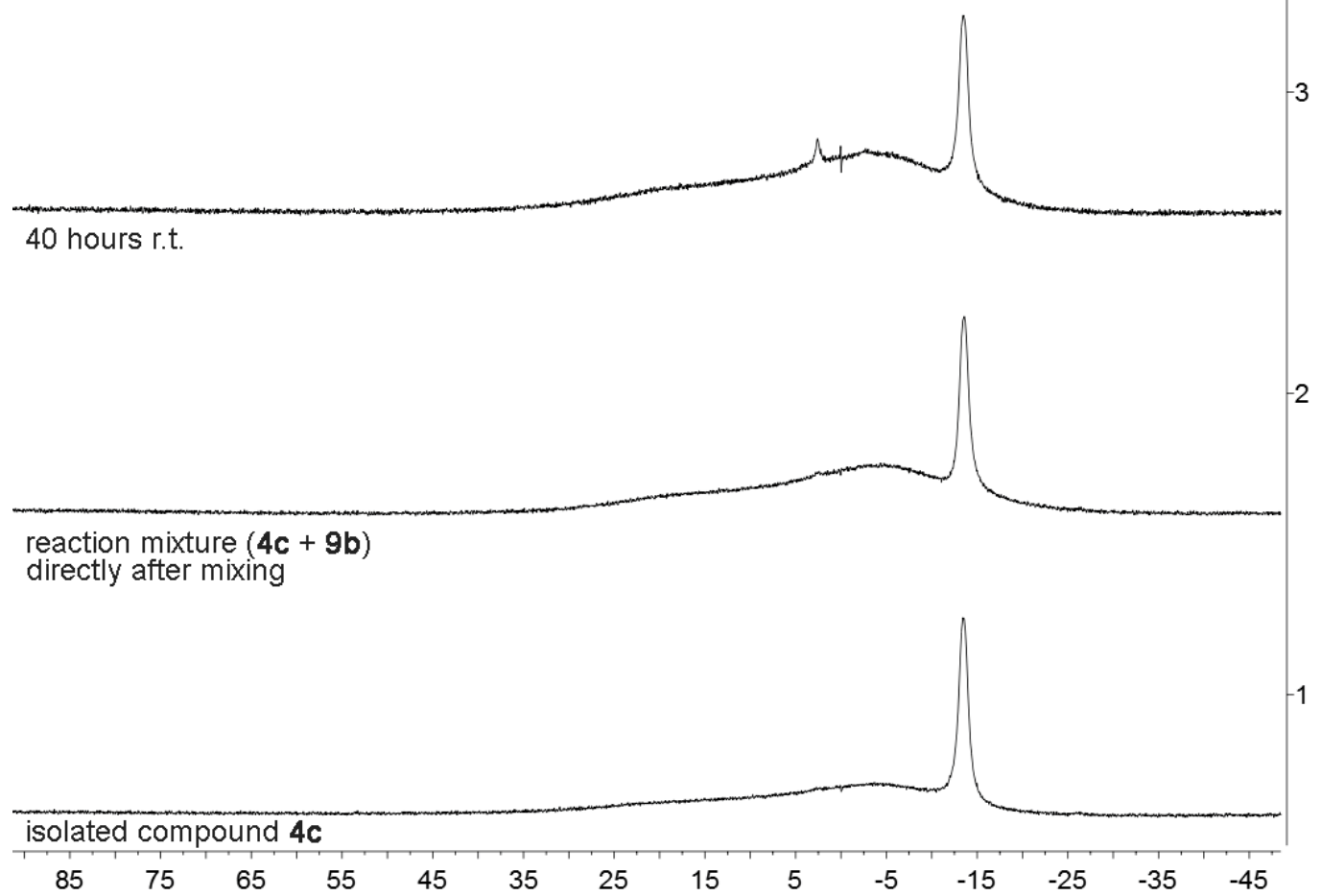

Figure S66. ${ }^{11} \mathbf{B}\left\{{ }^{1} \mathbf{H}\right\}$ NMR $\left(160 \mathrm{MHz}, 299 \mathrm{~K}, \mathrm{C}_{6} \mathrm{D}_{6}\right)$ spectra of (1) isolated compound $\mathbf{4 c}$ and $(2,3)$ the reaction mixture of the reaction of compounds $\mathbf{4 c}$ with $\mathbf{9 b}$

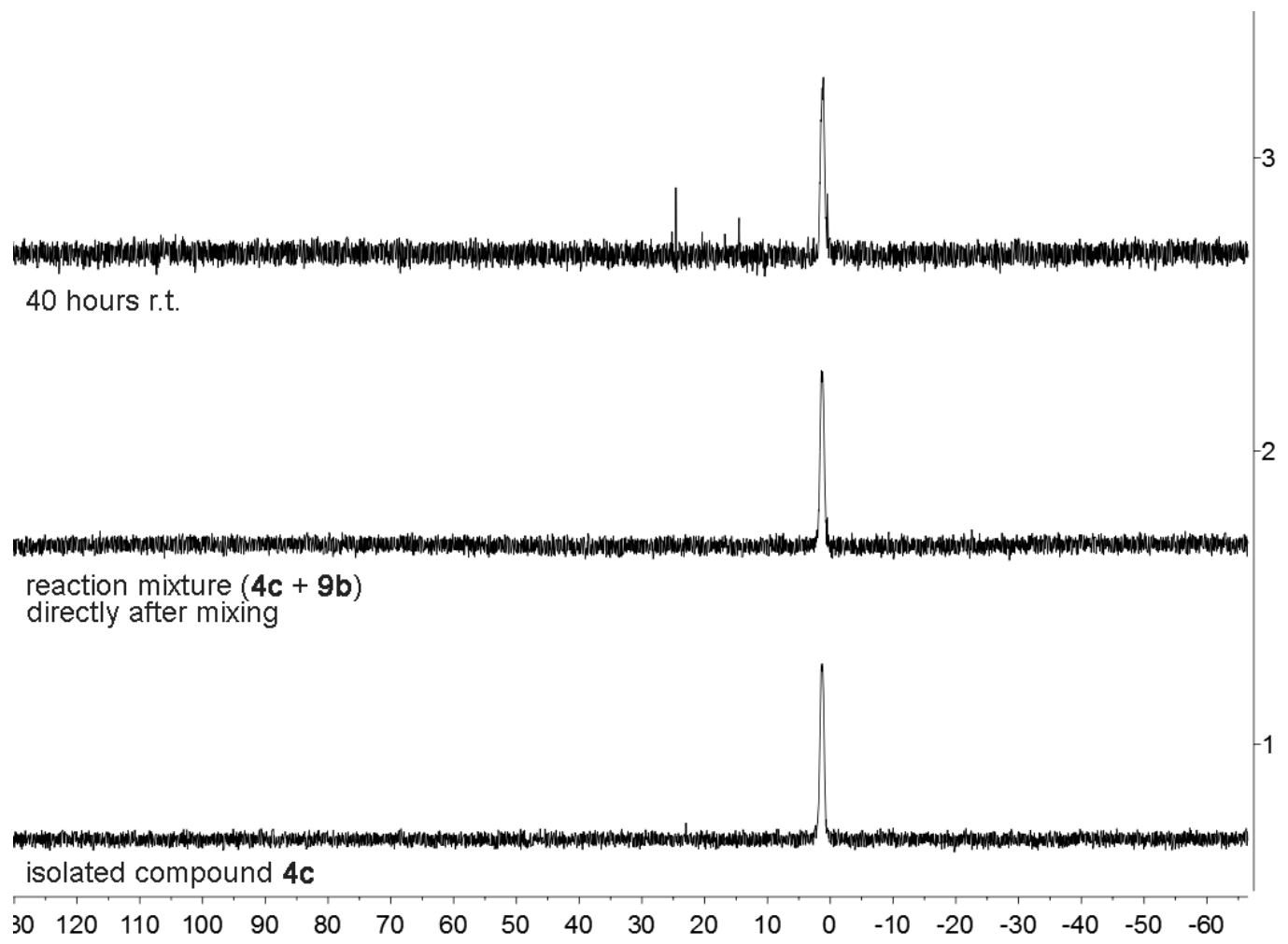

Figure S67. ${ }^{31} \mathbf{P}\left\{{ }^{1} \mathbf{H}\right\}$ NMR $\left(202 \mathrm{MHz}, 299 \mathrm{~K}, \mathrm{C}_{6} \mathrm{D}_{6}\right)$ spectra of (1) isolated compound $\mathbf{4 c}$ and $(2,3)$ the reaction mixture of the reaction of compounds $\mathbf{4 c}$ with $\mathbf{9 b}$ 
Control experiments: heating of compounds 8b, 10a, and 11a in $\mathrm{C}_{6} \mathrm{D}_{6}$ (NMR scale)

A solution of compound $\mathbf{8 b}(0.03 \mathrm{mmol}, 24.8 \mathrm{mg})$ [10a $(0.03 \mathrm{mmol}, 24.8 \mathrm{mg}), \mathbf{1 1 a}(0.03$ mmol, $19.6 \mathrm{mg})]$ in $\mathrm{C}_{6} \mathrm{D}_{6}(0.5 \mathrm{~mL})$ was transferred to a NMR tube, which was sealed immediately. Then the respective sample was heated and monitored by NMR spectroscopy.

\section{1) Heating of compound $8 \boldsymbol{b}$}

Comment: $\quad$ NMR spectra showed no change after heating a solution of compound 8b in $\mathrm{C}_{6} \mathrm{D}_{6}$ at $60{ }^{\circ} \mathrm{C}$ for 6 hours. Subsequent heating at $90{ }^{\circ} \mathrm{C}$ for 26 hours gave a complex mixture.

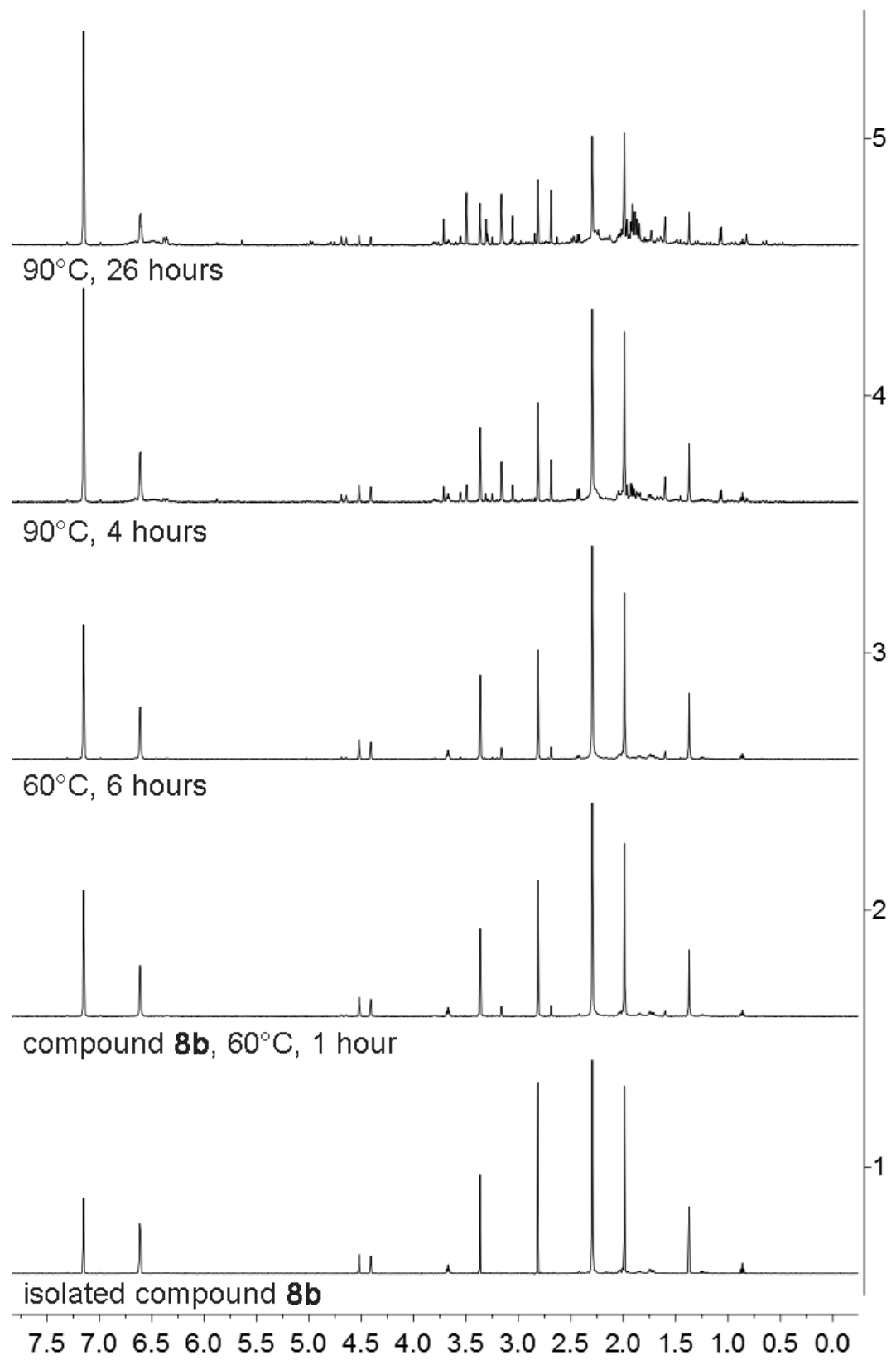

Figure S68. ${ }^{1} \mathbf{H}$ NMR $\left(500 \mathrm{MHz}, 299 \mathrm{~K}, \mathrm{C}_{6} \mathrm{D}_{6}\right)$ spectra (1) of isolated compound $\mathbf{8 b}$ and (2 to 5) after heating compound $\mathbf{8 b}$ 


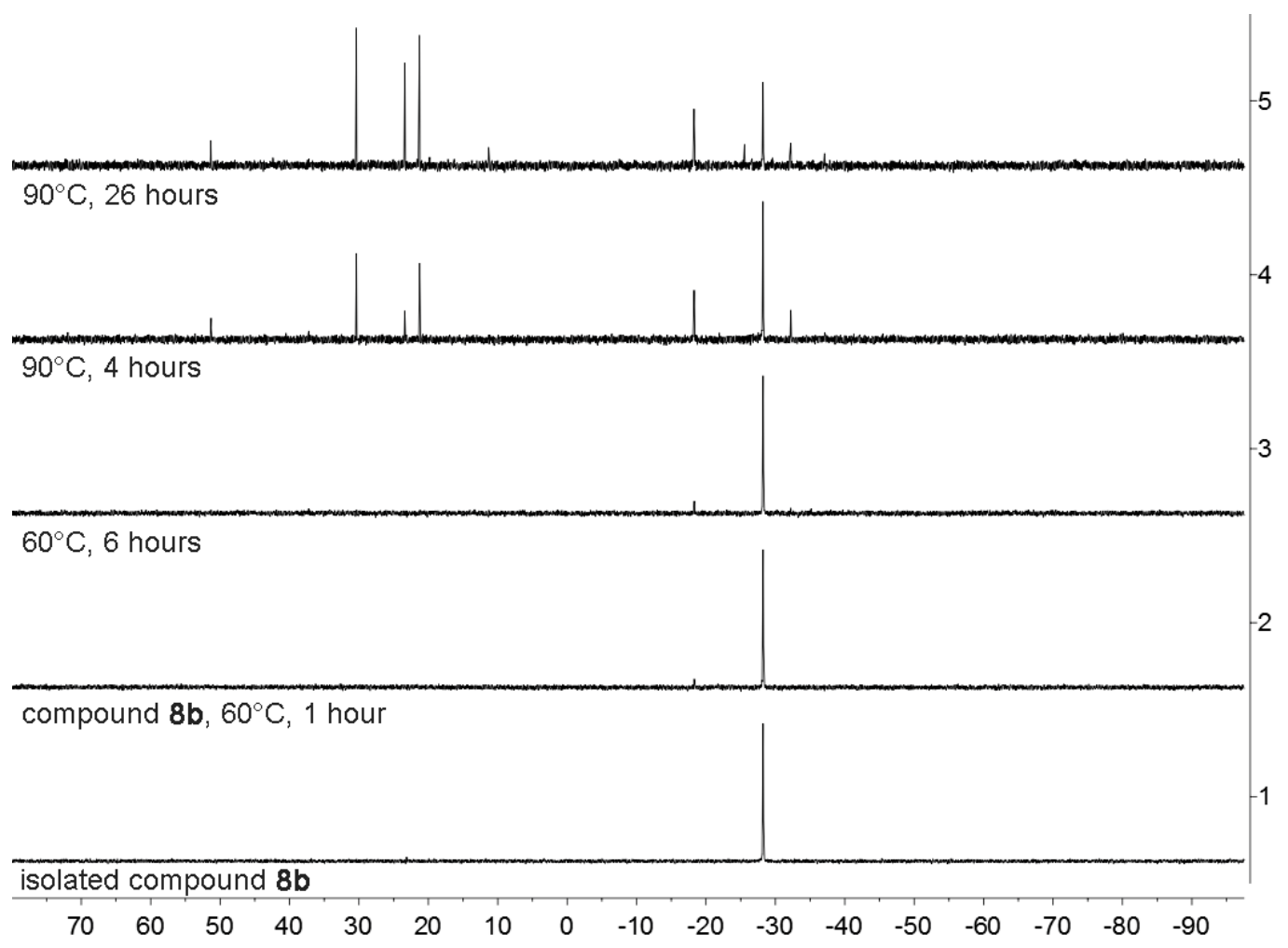

Figure S69. ${ }^{31} \mathbf{P}\left\{{ }^{1} \mathbf{H}\right\}$ NMR $\left(202 \mathrm{MHz}, 299 \mathrm{~K}, \mathrm{C}_{6} \mathrm{D}_{6}\right)$ spectra (1) of isolated compound $\mathbf{8 b}$ and (2 to 5) after heating compound $\mathbf{8 b}$

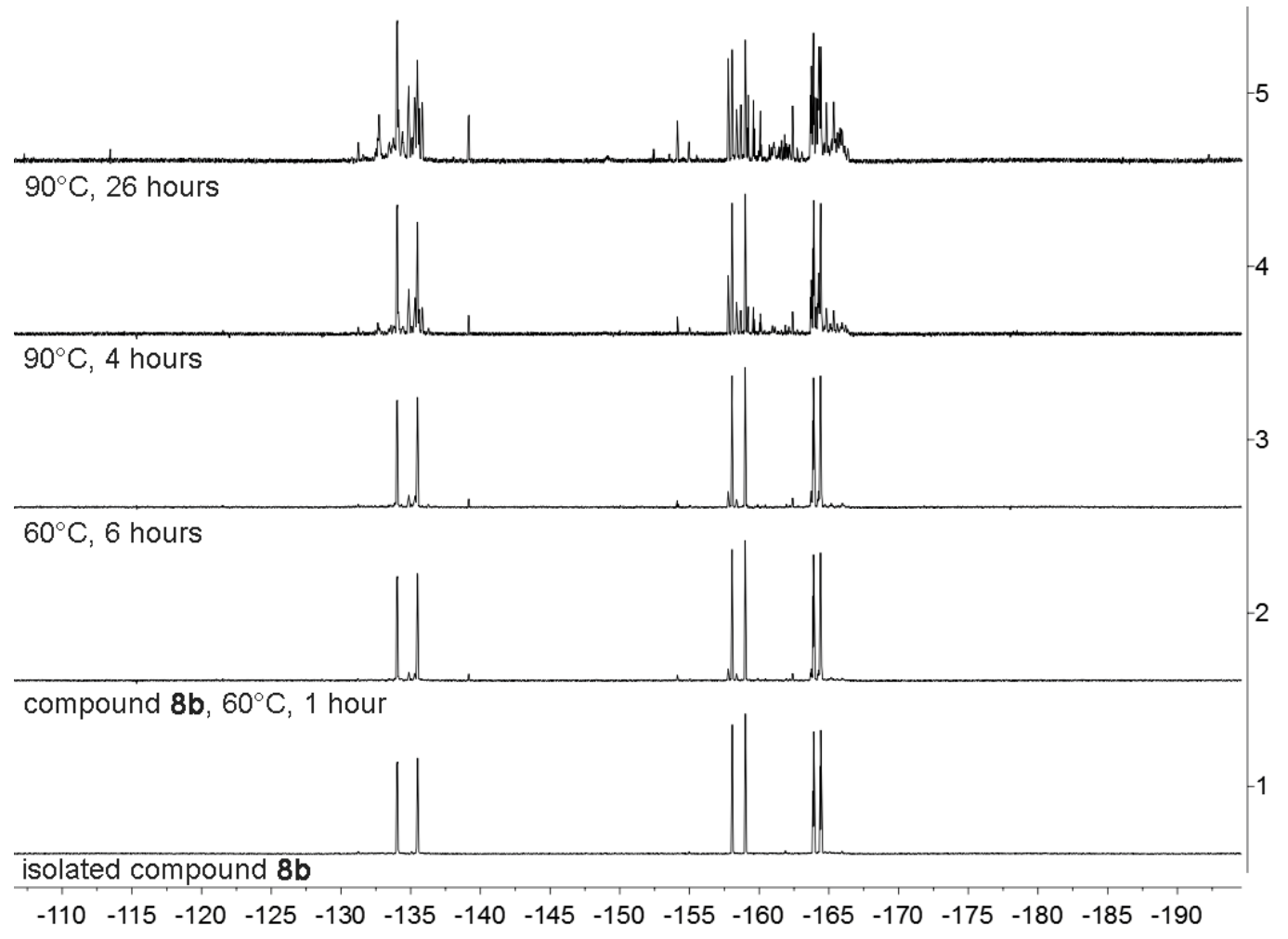

Figure S70. ${ }^{19} \mathbf{F}$ NMR (470 MHz, $\left.299 \mathrm{~K}, \mathrm{C}_{6} \mathrm{D}_{6}\right)$ spectra (1) of isolated compound $\mathbf{8 b}$ and (2 to 5) after heating compound $\mathbf{8 b}$ 


\section{2) Heating of compound $10 a$}

Comment: $\quad$ NMR spectra showed that heating a solution of compound 10a in $\mathrm{C}_{6} \mathrm{D}_{6}$ at $90{ }^{\circ} \mathrm{C}$ for 4 hours gave a major compound $\left[\delta^{31} \mathrm{P}\left\{{ }^{1} \mathrm{H}\right\}: 42.2\left(v_{1 / 2} \sim 400\right.\right.$ $\mathrm{Hz})]$ which could not been identified yet.

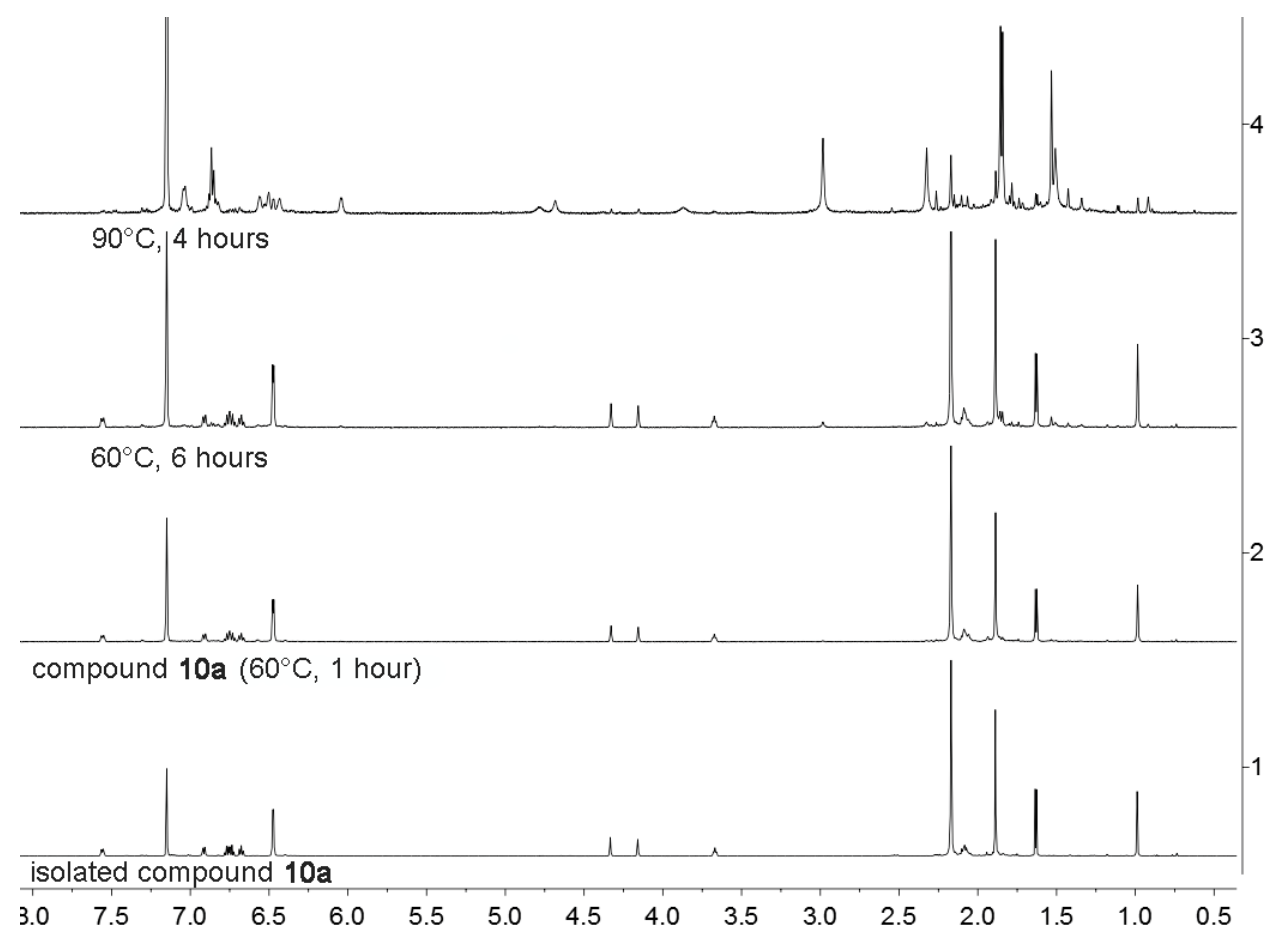

Figure S71. (1) ${ }^{1} \mathbf{H}$ NMR $\left(600 \mathrm{MHz}, 299 \mathrm{~K}, \mathrm{C}_{6} \mathrm{D}_{6}\right)$ spectrum of isolated compound 10a and (2 to 4) ${ }^{\mathbf{1}} \mathbf{H}$ NMR (500 MHz, $299 \mathrm{~K}, \mathrm{C}_{6} \mathrm{D}_{6}$ ) spectrum after heating compound 10a

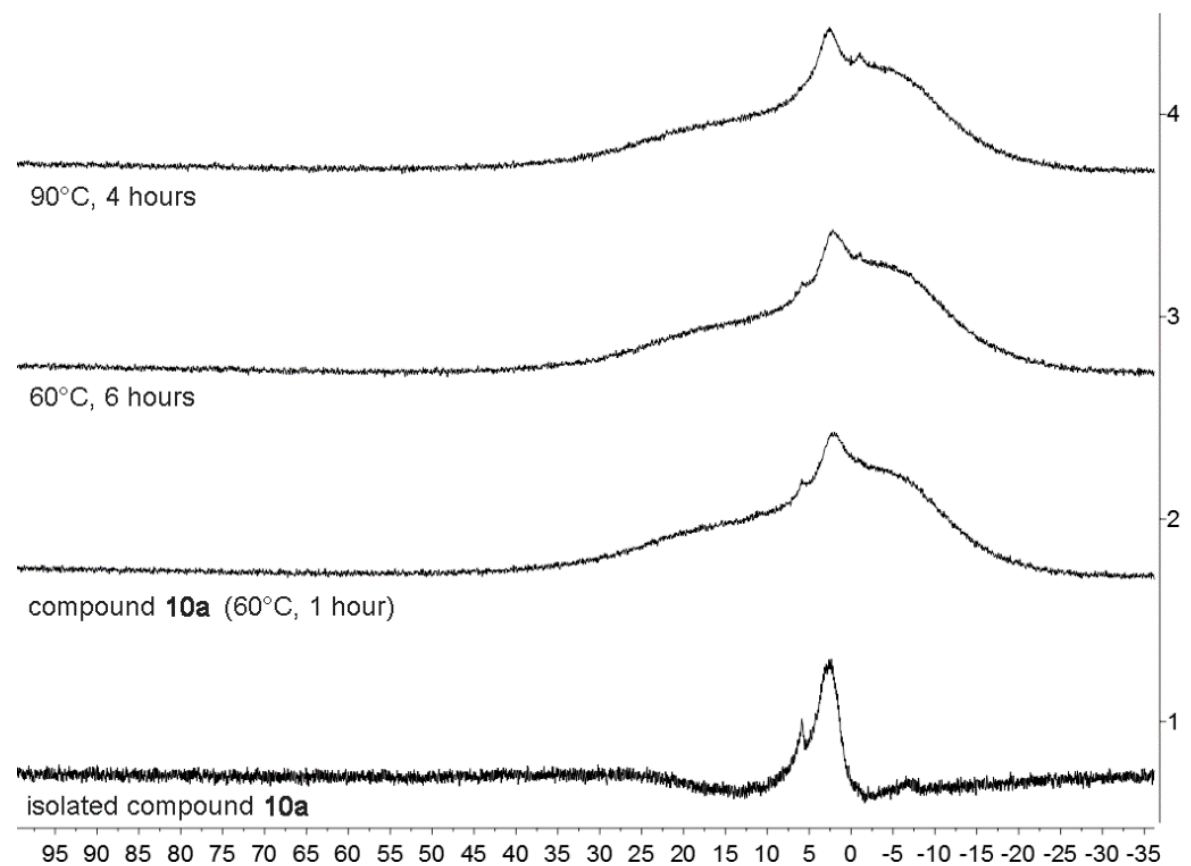

Figure S72. (1) ${ }^{11} \mathbf{B}\left\{{ }^{1} \mathbf{H}\right\}$ NMR (192 MHz, $\left.299 \mathrm{~K}, \mathrm{C}_{6} \mathrm{D}_{6}\right)$ spectrum of isolated compound 10a and (2 to 4) ${ }^{\mathbf{1 1}} \mathbf{B}\left\{{ }^{1} \mathbf{H}\right\}$ NMR (160 MHz, $\left.299 \mathrm{~K}, \mathrm{C}_{6} \mathrm{D}_{6}\right)$ spectrum after heating compound $\mathbf{1 0 a}$ 


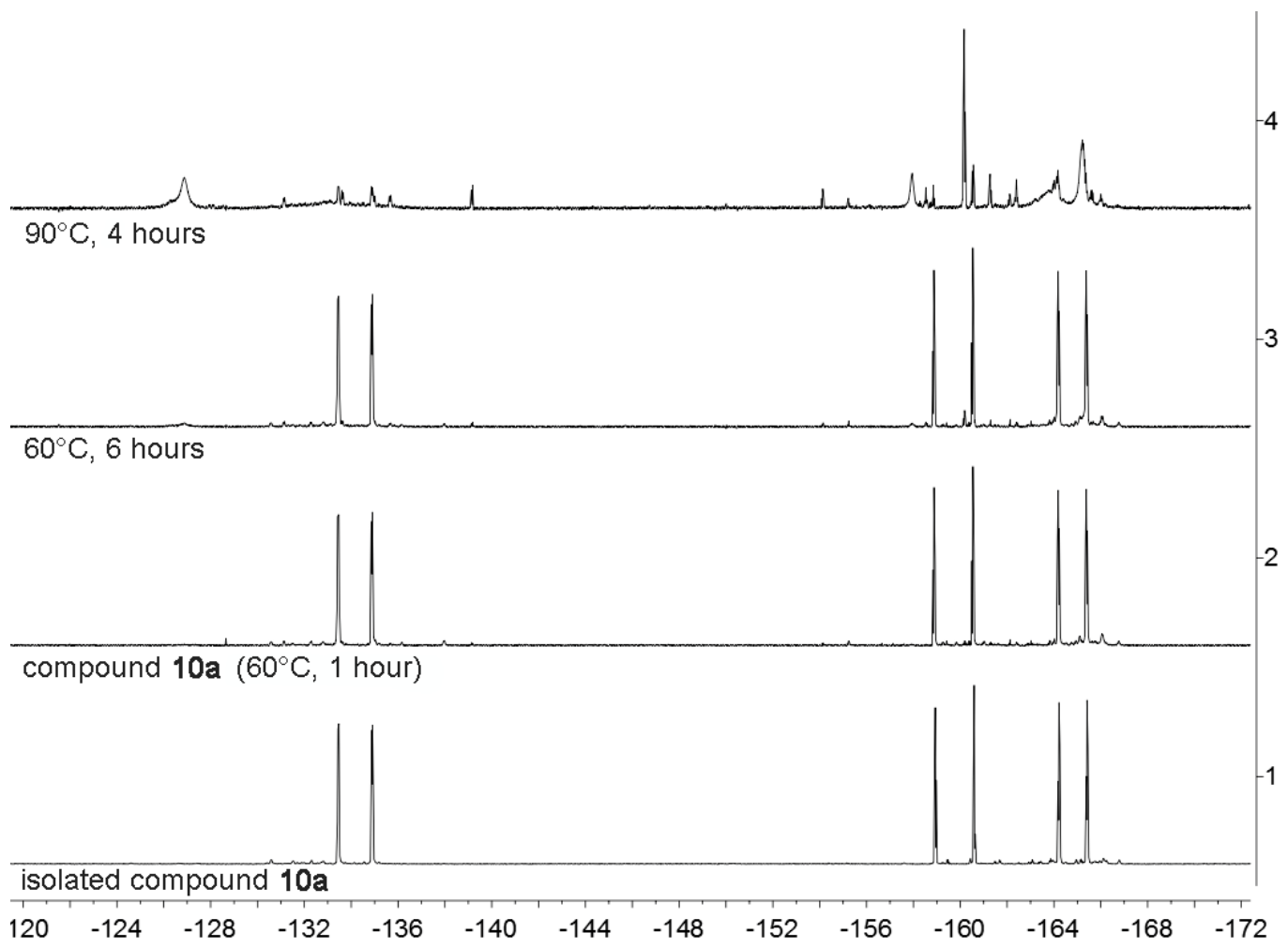

Figure S73. (1) ${ }^{19}$ F NMR (564 MHz, $\left.299 \mathrm{~K}, \mathrm{C}_{6} \mathrm{D}_{6}\right)$ spectrum of isolated compound 10a and (2 to 4) ${ }^{19} \mathbf{F}$ NMR $\left(470 \mathrm{MHz}, 299 \mathrm{~K}, \mathrm{C}_{6} \mathrm{D}_{6}\right)$ spectrum after heating compound 10a

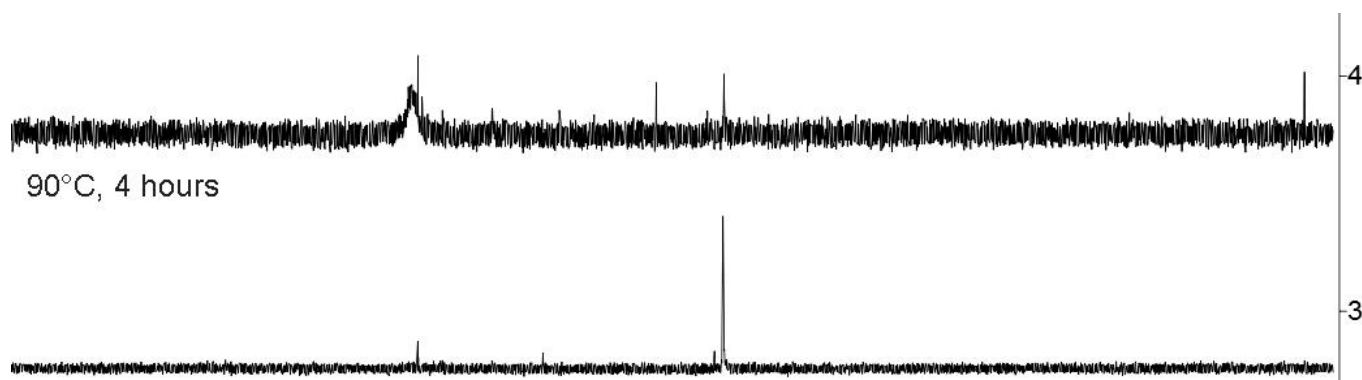

$60^{\circ} \mathrm{C}, 6$ hours

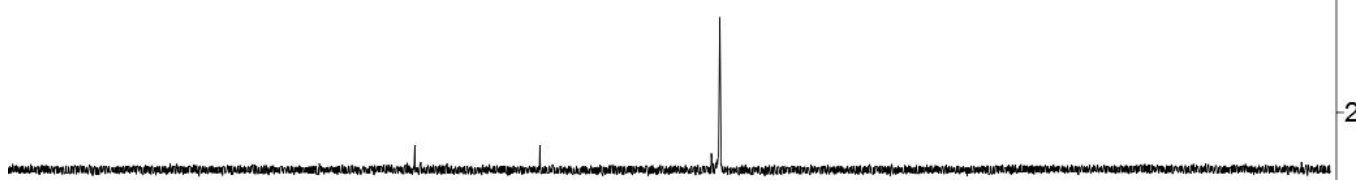

compound $10 \mathrm{a}\left(60^{\circ} \mathrm{C}, 1\right.$ hour $)$

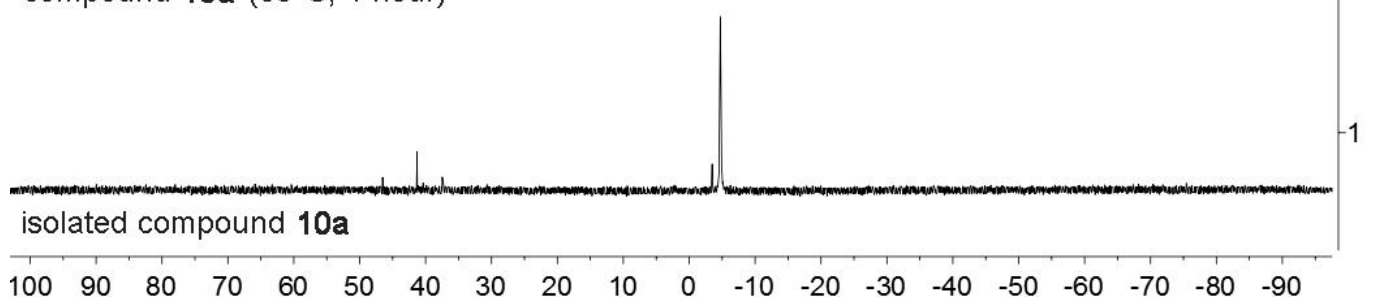

Figure S74. (1) ${ }^{31} \mathbf{P}\left\{{ }^{1} \mathbf{H}\right\}$ NMR (243 MHz, $\left.299 \mathrm{~K}, \mathrm{C}_{6} \mathrm{D}_{6}\right)$ spectrum of isolated compound 10a and (2 to 4) ${ }^{31} \mathbf{P}\left\{{ }^{1} \mathbf{H}\right\}$ NMR (202 MHz, $\left.299 \mathrm{~K}, \mathrm{C}_{6} \mathrm{D}_{6}\right)$ spectrum after heating compound 10a 


\section{3) Heating of compound 11a}

Comment: $\quad$ NMR spectra showed that heating a solution of compound 11a in $\mathrm{C}_{6} \mathrm{D}_{6}$ at $60{ }^{\circ} \mathrm{C}$ for 9 hours gave two major products $\left[\delta^{31} \mathrm{P}\left\{{ }^{1} \mathrm{H}\right\}: 47.4(68 \mathrm{~mol} \%)\right.$, 24.0 (32 $\mathrm{mol} \%)]$ which were tentatively assigned as $\mathbf{1 6}\left(\mathbf{P}^{t} \mathbf{B u} \mathbf{u}_{2}\right)$ and $\mathbf{1 8}+$ $\mathrm{HC}_{6} \mathrm{~F}_{5}\left[\delta^{1} \mathrm{H}\left(\mathrm{HC}_{6} \mathrm{~F}_{5}\right)=5.81(\mathrm{~m}) ; \delta^{19} \mathrm{~F}\left(\mathrm{HC}_{6} \mathrm{~F}_{5}\right)=-139.2(\mathrm{~m}, 2 \mathrm{~F}, o),-154.2\right.$ $\left.\left(\mathrm{tm},{ }^{3} \mathrm{~J}_{\mathrm{FF}}=20.7 \mathrm{~Hz}, 1 \mathrm{~F}, p\right),-162.4(\mathrm{~m}, 2 \mathrm{~F}, m)\right]$
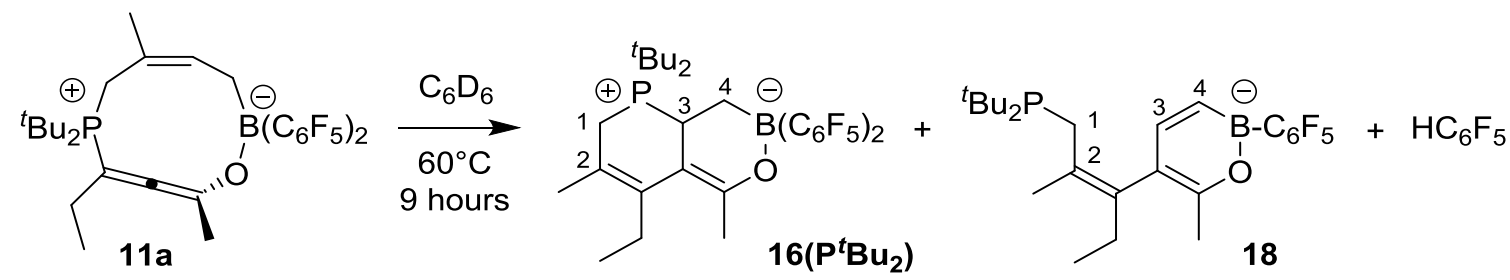

Scheme S9

Table S1. Selected NMR data ${ }^{a}$ in comparison with isolated compound $\mathbf{1 6}$ (see above).

\begin{tabular}{llll}
\hline & $\mathbf{1 6}$ & $\mathbf{1 6}\left(\mathbf{P}^{t} \mathbf{B u} 2\right)$ & $\mathbf{1 8}$ \\
& $\begin{array}{l}\mathrm{Ph}_{2} \mathrm{P} \\
\text { (isolated) }\end{array}$ & $\begin{array}{l}{ }^{t} \mathrm{Bu}_{2} \mathrm{P} \\
(68 \mathrm{~mol} \%)\end{array}$ & $\begin{array}{l}{ }^{t} \mathrm{Bu}_{2} \mathrm{P} \\
(32 \mathrm{~mol} \%)\end{array}$ \\
\hline$\delta^{31} \mathrm{P}$ & 27.3 & 47.4 & 24.0 \\
$\delta^{19} \mathrm{~F}$, ortho & $-134.5 /-136.6$ & $-133.8 /-135.2$ & -132.7 \\
para & $-162.5 /-162.9$ & $-161.7 /-161.9$ & -152.2 \\
meta & $-166.5 /-166.6$ & $-165.6 /-165.7$ & -163.0 \\
$\delta^{11} \mathrm{~B}$ & -3.1 & -2.9 & 36.2 \\
$\delta^{1} \mathrm{H}$ & & & \\
$\mathrm{PCH}{ }_{2}$ & $3.35 / 2.79$ & $1.87 / 1.22$ & $2.42 / 3.32$ \\
$\mathrm{BCH}{ }_{2}$ & $1.45 / 0.83$ & $2.02 / 1.81$ & $7.40^{\mathrm{b}}$ \\
$\mathrm{CH}$ & 3.39 & 2.96 & $6.92^{\mathrm{c}}$ \\
$\mathrm{Et}$ & $2.45 / 2.18$ & $2.14 / 1.93$ & $2.35 / 2.33$ \\
$\mathrm{Et}$ & 0.72 & 0.58 & 0.87 \\
$\mathrm{O}-\mathrm{C} \mathrm{Me}$ & $1.79 \mathrm{~g}$ & $1.94^{\mathrm{h}}$ & 2.12 \\
$\mathrm{Me}$ & 1.59 & 1.36 & 1.76 \\
$\delta^{13} \mathrm{C}^{\mathrm{d}, \mathrm{e}}$ & & & \\
$=\mathrm{CO}$ & $159.3(11.5)$ & $159.6(11.4)$ & $156.3(1.3)$ \\
$=\mathrm{CEt}$ & $145.0(12.0)$ & $144.9(8.2)$ & $132.0(5.0)$ \\
$=\mathrm{CMe}$ & $117.6(11.1)$ & $116.7(10.7)$ & $133.6(11.4)$ \\
$=\mathrm{C}$ & $97.9(11.2)$ & $101.6(11.5)$ & 124.5 \\
$\mathrm{PCH}{ }_{2}$ & $28.1^{\mathrm{e}}(49.5)$ & $20.9(41.7)$ & $27.4(26.0)$ \\
$\mathrm{BCH}{ }_{2}$ & $20.2^{\mathrm{f}}$ & $19.5^{\mathrm{f}}$ & $125.2^{\mathrm{b}, \mathrm{f}}$ \\
$\mathrm{CH}$ & $33.0(45.8)$ & $34.3(39.1)$ & $152.6^{\mathrm{c}}(1.3)$ \\
\hline
\end{tabular}

${ }^{\mathrm{a}}$ in $\mathrm{CD}_{2} \mathrm{Cl}_{2}$ at $299 \mathrm{~K} .{ }^{\mathrm{b}}=\mathrm{CHB}\left(\mathrm{d},{ }^{2} J_{\mathrm{HH}}=11.4 \mathrm{~Hz}\right) .{ }^{\mathrm{c}}=\mathrm{CH}\left(\mathrm{br} \mathrm{d},{ }^{2} J_{\mathrm{HH}}=11.4 \mathrm{~Hz}\right) .{ }^{\mathrm{d}}$ supported by ghsqc and ghmbc experiments. ${ }^{\mathrm{e}}{ }_{\mathrm{XC}}: \mathrm{X}:{ }^{31} \mathrm{P},{ }^{19} \mathrm{~F}$ in ( ). ${ }^{\mathrm{f}}$ broad. ${ }^{\mathrm{g}} \mathrm{dd},{ }^{5} J_{\mathrm{PH}}$ $=5.6 \mathrm{~Hz},{ }^{5} J_{\mathrm{HH}}=1.6 \mathrm{~Hz} \cdot{ }^{\mathrm{h}} \mathrm{dd},{ }^{\mathrm{n}} J_{\mathrm{XH}}=6.2,1.1 \mathrm{~Hz}$. 


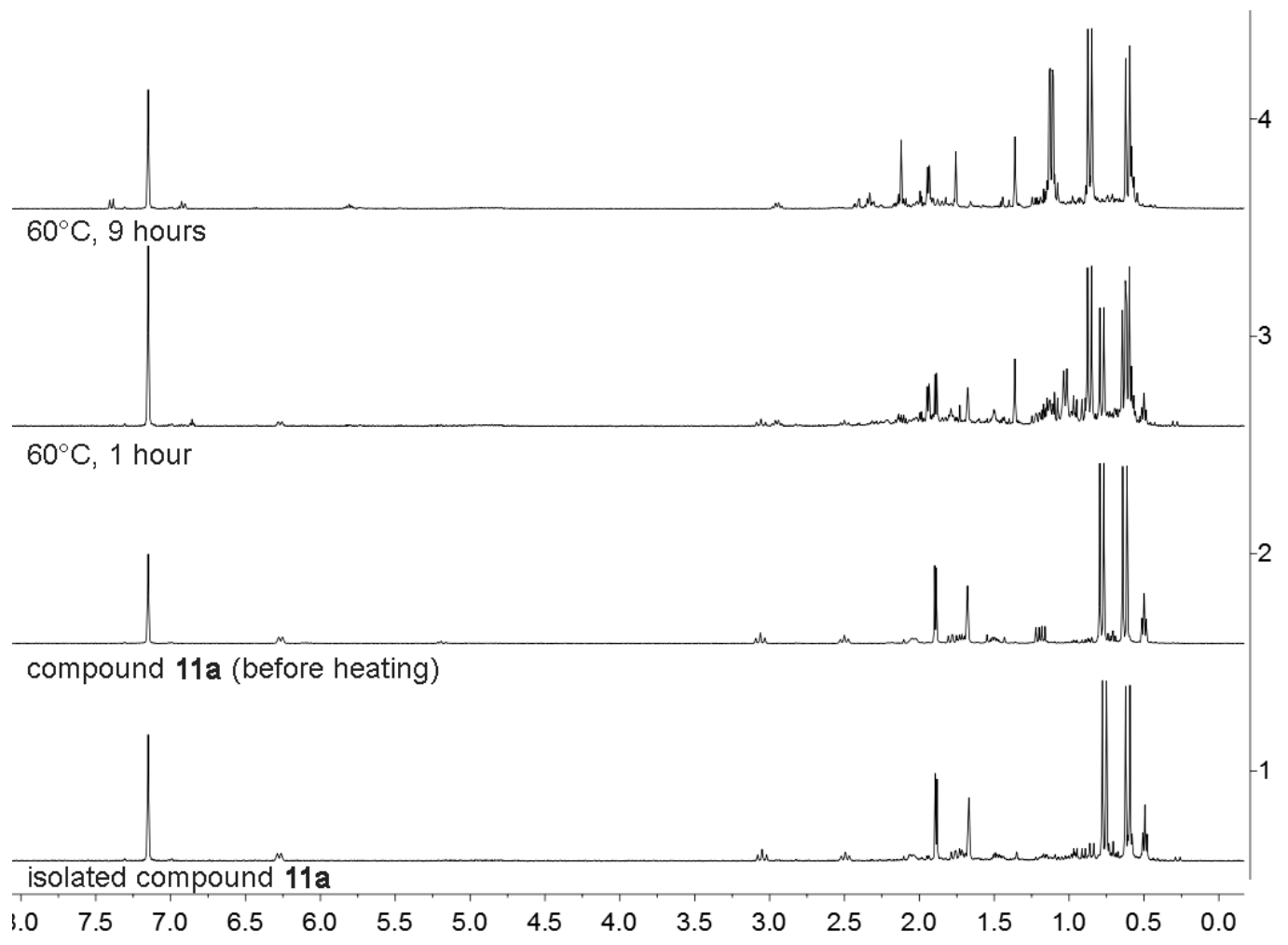

Figure S75. ${ }^{1} \mathbf{H}$ NMR (500 MHz, $\left.299 \mathrm{~K}, \mathrm{C}_{6} \mathrm{D}_{6}\right)$ spectra (1) of isolated compound 11a and (2 to 4 ) after heating compound 11a

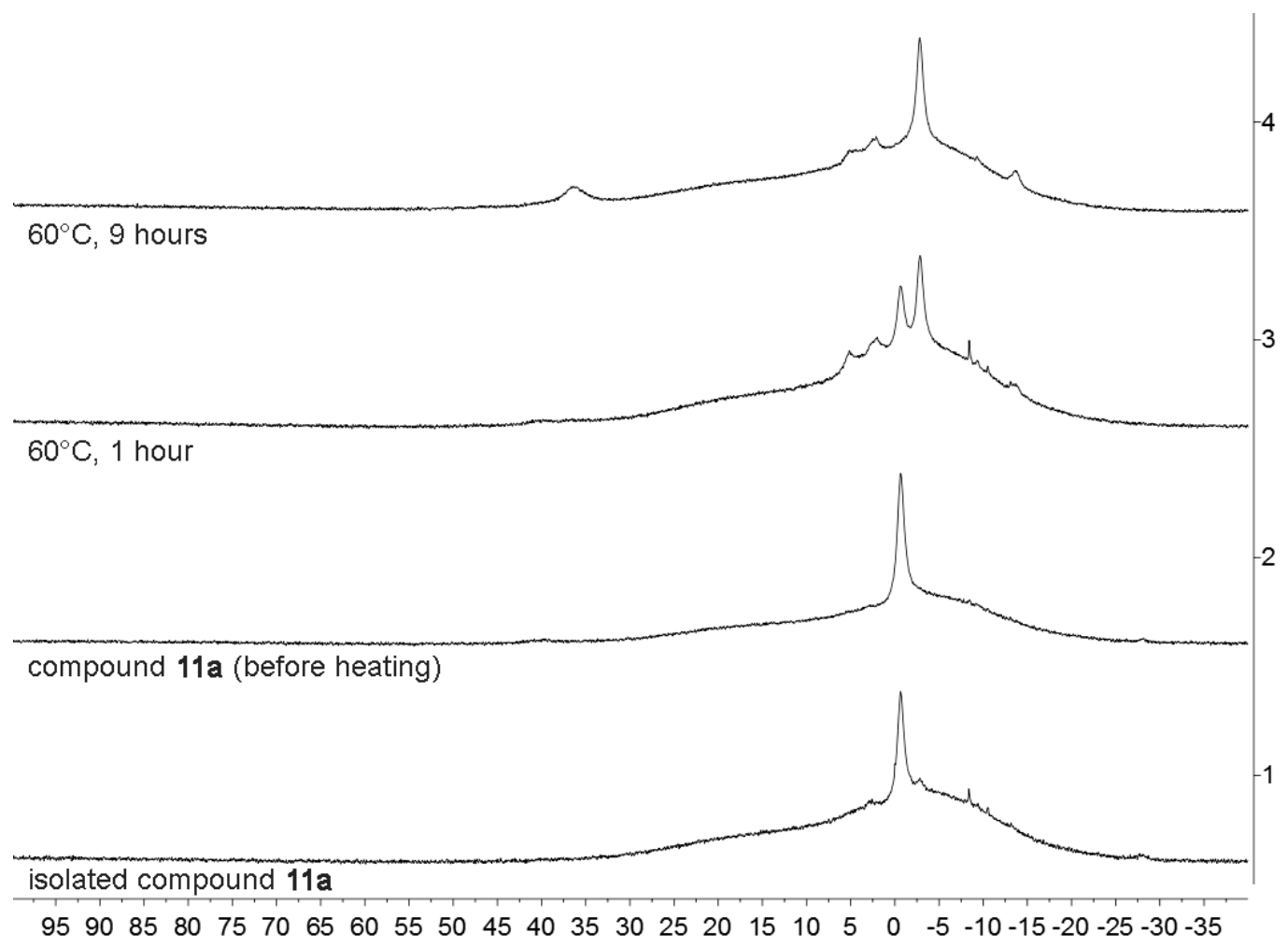

Figure S76. ${ }^{11} \mathbf{B}\left\{{ }^{1} \mathbf{H}\right\}$ NMR $\left(160 \mathrm{MHz}, 299 \mathrm{~K}, \mathrm{C}_{6} \mathrm{D}_{6}\right)$ spectra (1) of isolated compound 11a and (2 to 4 ) after heating compound 11a 


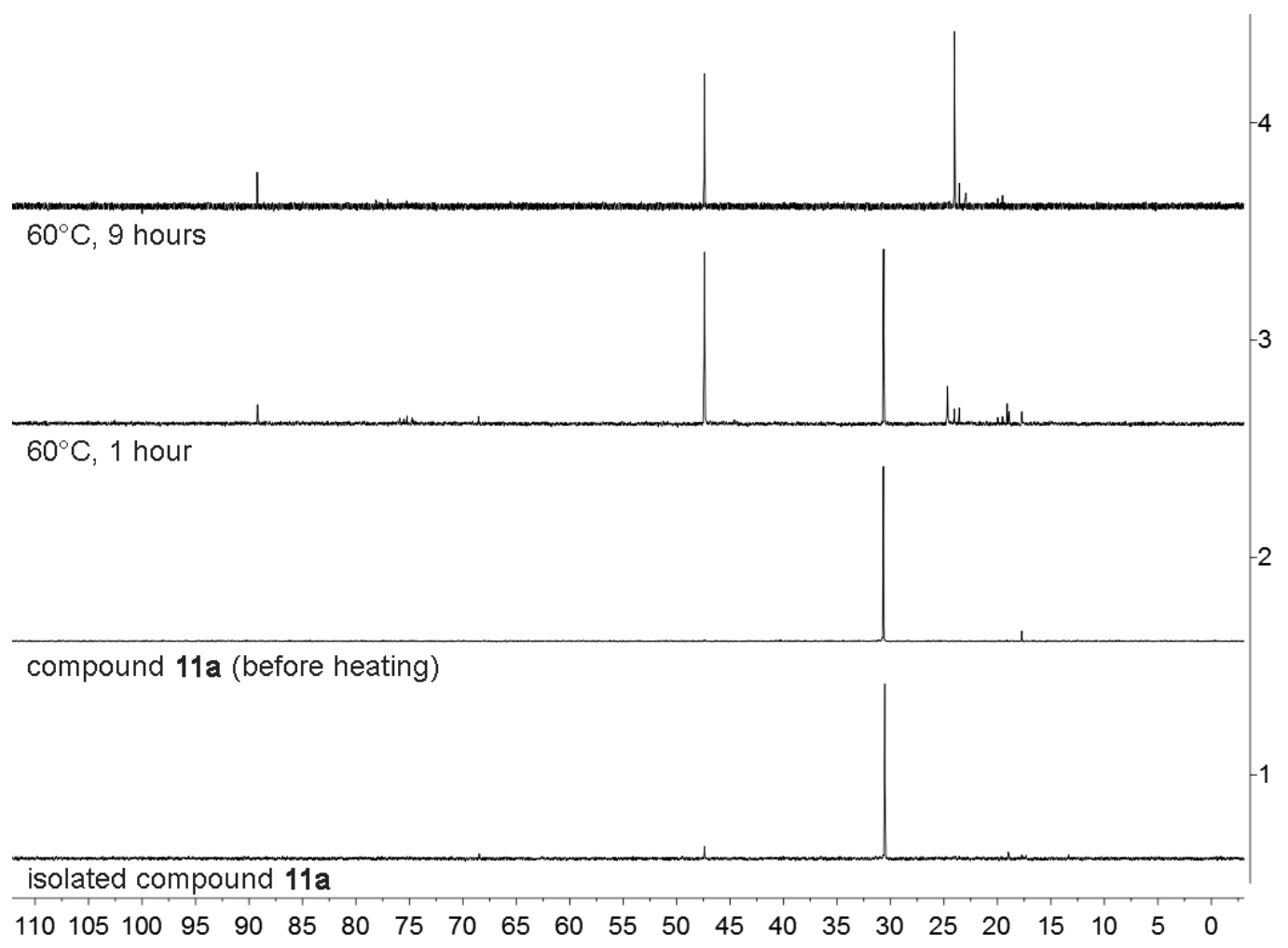

Figure S77. ${ }^{31} \mathbf{P}\left\{{ }^{1} \mathbf{H}\right\}$ NMR $\left(202 \mathrm{MHz}, 299 \mathrm{~K}, \mathrm{C}_{6} \mathrm{D}_{6}\right)$ spectra (1) of isolated compound 11a and (2 to 4 ) after heating compound 11a

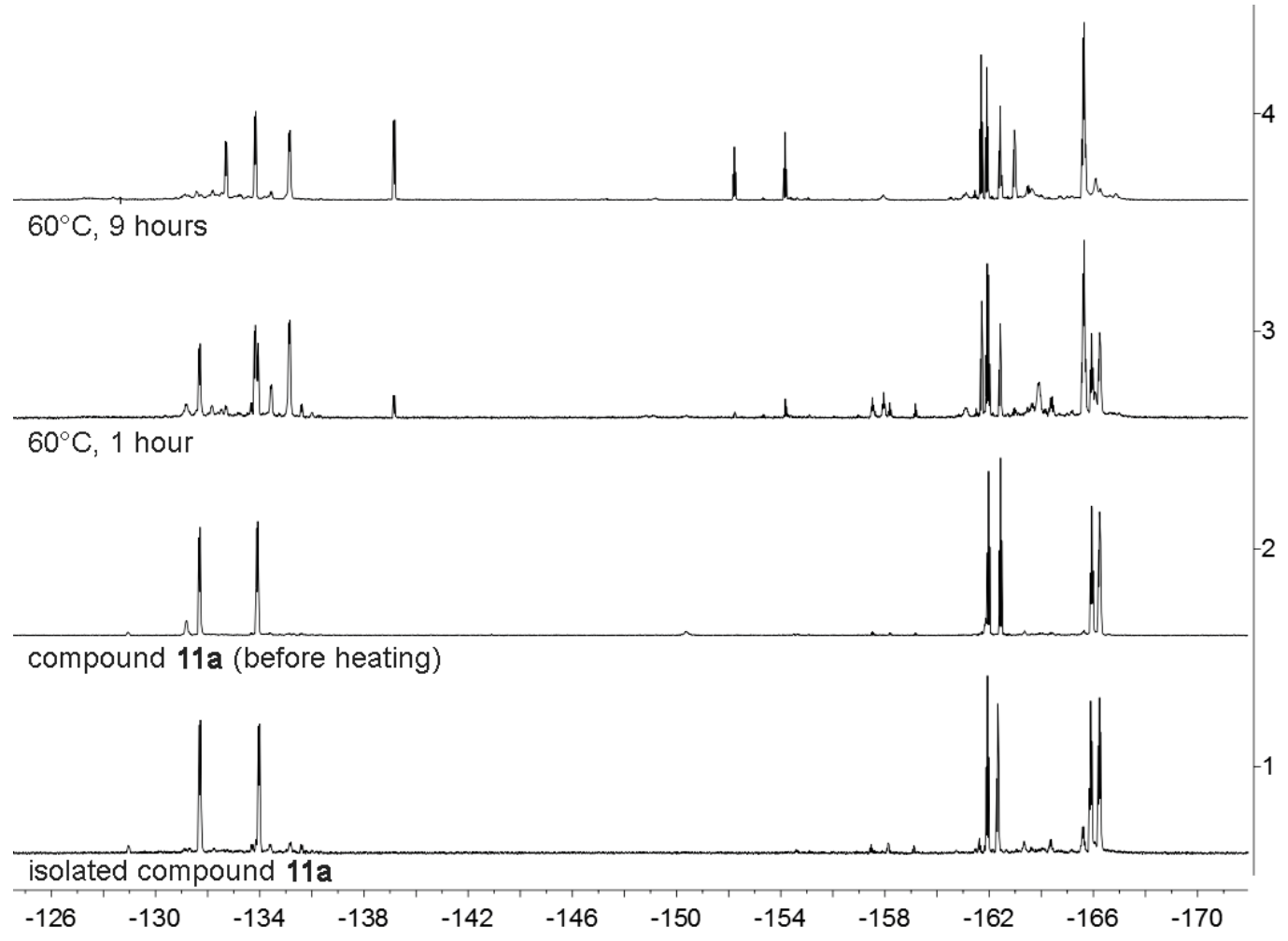

Figure S78. ${ }^{19}$ F NMR $\left(470 \mathrm{MHz}, 299 \mathrm{~K}, \mathrm{C}_{6} \mathrm{D}_{6}\right)$ spectra (1) of isolated compound 11a and ( 2 to 4$)$ after heating compound 11a 


\section{DFT Calculations}

\section{Methods}

All calculations were performed with the TURBOMOLE $7.0^{1}$ and ORCA 3.0.3 (only for PES scans) programs. The structures were optimized without any geometry constraints using the TPSS functional ${ }^{3}$ and an atompairwise dispersion correction (D3). ${ }^{4}$ A flexible triple zeta basis set (def2-TZVP) ${ }^{5}$ was used in all calculations (def2-SVP in the PES scans). For the calculation of zero point vibrational energies and free enthalpy contributions, a rotor approximation was applied for vibrational modes with wave numbers below $100 \mathrm{~cm}^{-1}{ }^{6}$ Single point calculations were performed with TPSS-D3 and the hybrid functional PW6B95(-D3). ${ }^{7}$ Free energies of solvation were obtained with the COSMO-RS model ${ }^{8}$ for $298 \mathrm{~K}$ using pentane as solvent.

\section{Results}

Table S2. DFT-calculated electronic energies (TPSS-D3 and PW6B95-D3) after geometry optimization with TPSS-D3. Zero point vibrational energies (ZPE), enthalpic and free energy corrections for $298 \mathrm{~K}(\mathrm{H}(298 \mathrm{~K})$, G(298K) with TPSS-D3). GsoLv (298K): COSMO-RS calculated free energy of solvation for $298 \mathrm{~K}$ in pentane. For all calculations the def2-TZVP basis set was used.

\begin{tabular}{ccccccc}
\hline & \multirow{2}{*}{ TPSS-D3 } & ZPE & $\mathrm{H}(298 \mathrm{~K})$ & $\mathrm{G}(298 \mathrm{~K})$ & $\mathrm{PW} 6 \mathrm{~B} 95-\mathrm{D} 3$ & $\mathrm{G}_{\text {SOLv }}(298 \mathrm{~K})$ \\
& {$\left[\mathrm{E}_{\mathrm{h}}\right]$} & {$[\mathrm{kcal} / \mathrm{mol}]$} & {$[\mathrm{kcal} / \mathrm{mol}]$} & {$[\mathrm{kcal} / \mathrm{mol}]$} & {$\left[\mathrm{E}_{\mathrm{h}}\right]$} & {$[\mathrm{kcal} / \mathrm{mol}]$} \\
\hline $\mathbf{4 b}$ & -2334.080516 & 289.496 & 314.266 & 250.625 & -2336.395262 & -11.671 \\
$\mathbf{5 b}$ & -533.392485 & 70.449 & 78.090 & 47.208 & -533.930166 & -3.197 \\
$\mathbf{( 4 b \cdot 5 b )}$ & -2867.456263 & 359.563 & 392.776 & 311.647 & -2870.303904 & -16.861 \\
9b & -308.797619 & 75.571 & 81.708 & 54.498 & -309.120594 & -2.182 \\
7d & -2867.492867 & 361.017 & 393.373 & 314.099 & -2870.343042 & -22.425 \\
TS 7d-13a & -2867.481301 & 360.616 & 392.445 & 314.330 & -2870.325889 & -19.857 \\
13a & -2867.507479 & 360.706 & 392.628 & 314.049 & -2870.351562 & -20.189 \\
TS 13a-8d & -2867.494697 & 361.308 & 393.100 & 315.363 & -2870.335547 & -17.258 \\
8d & -2867.532094 & 361.502 & 393.902 & 314.042 & -2870.384244 & -18.306 \\
12 & -2867.558134 & 363.153 & 394.586 & 317.372 & -2870.416554 & -18.593 \\
epi-12 & -2867.499064 & 362.786 & 394.155 & 317.533 & -2870.355202 & -20.932 \\
17 & -2867.558553 & 363.346 & 394.628 & 318.047 & -2870.417775 & -20.782 \\
\hline 11a & -2642.914211 & 367.136 & 397.621 & 322.642 & -2645.550205 & -21.264 \\
13a-analogue & -2642.893052 & 367.065 & 396.903 & 323.034 & -2645.517117 & -21.610 \\
8d-analogue & -2642.922403 & 366.965 & 397.504 & 322.200 & -2645.557525 & -16.017 \\
12-analogue & -2642.916554 & 367.508 & 397.339 & 323.194 & -2645.552361 & -19.027 \\
\hline
\end{tabular}


Table S3. DFT-calculated relative energies and free energies of intermediates in the phospha-Claisen reaction (Scheme 6) with respect to the reactants $\mathbf{4 b + 5 b}$.

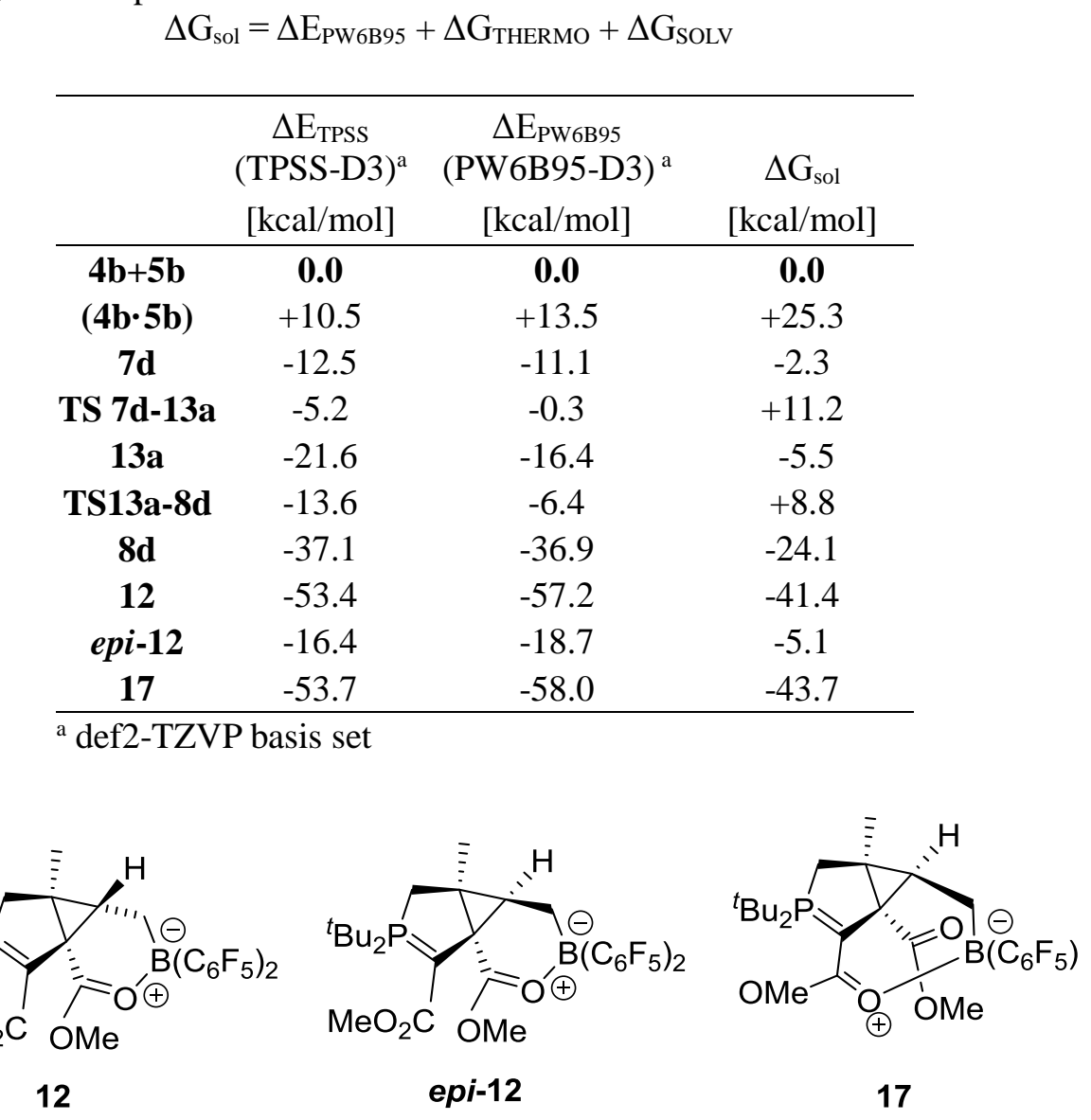

Table S4. DFT-calculated relative energies and free energies of intermediates in the phospha-Claisen reaction of $4 b$ and $9 b$ (Scheme 5) with respect to the reactants $4 b+9 b$.

$$
\Delta \mathrm{G}_{\mathrm{sol}}=\Delta \mathrm{E}_{\mathrm{PW} 6 \mathrm{~B} 95}+\Delta \mathrm{G}_{\mathrm{THERMO}}+\Delta \mathrm{G}_{\mathrm{SOLV}}
$$

\begin{tabular}{|c|c|c|c|}
\hline & $\begin{array}{c}\Delta \mathrm{E}_{\mathrm{TPSS}} \\
\left(_{\mathrm{TPSS}-\mathrm{D} 3)^{\mathrm{a}}}\right. \\
{[\mathrm{kcal} / \mathrm{mol}]}\end{array}$ & $\begin{array}{c}\Delta \mathrm{E}_{\mathrm{PW} 6 \mathrm{~B} 95} \\
\left(^{\mathrm{PW} 6 \mathrm{~B} 95-\mathrm{D} 3)^{\mathrm{a}}}\right. \\
{[\mathrm{kcal} / \mathrm{mol}]}\end{array}$ & $\begin{array}{c}\Delta \mathrm{G}_{\mathrm{sol}} \\
{[\mathrm{kcal} / \mathrm{mol}]}\end{array}$ \\
\hline $4 b+9 b$ & $\mathbf{0 . 0}$ & $\mathbf{0 . 0}$ & $\mathbf{0 . 0}$ \\
\hline $11 a$ & -22.6 & -21.6 & -11.5 \\
\hline 13a-analogue & -9.4 & -0.8 & 9.4 \\
\hline 8d-analogue & -27.8 & -26.2 & -11.2 \\
\hline 12-analogue & -24.1 & -22.9 & -10.0 \\
\hline
\end{tabular}

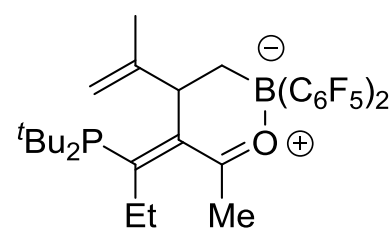

"8d-analogue"

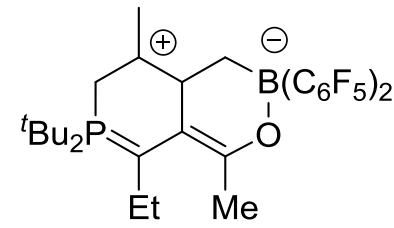

"13a-analogue"

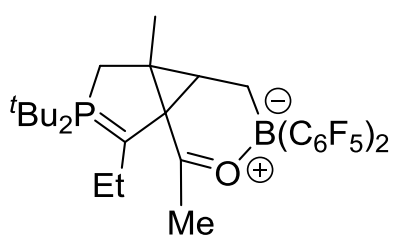

"12-analogue" 
Figure S79: DFT-optimized structures of intermediates in the phospha-Claisen reaction of $\mathbf{4 b}$, (TPSS-D3/def2-TZVP). Bond lengths in $\AA$. (Colors: orange $=$ P, yellow $=F$, rose = B)

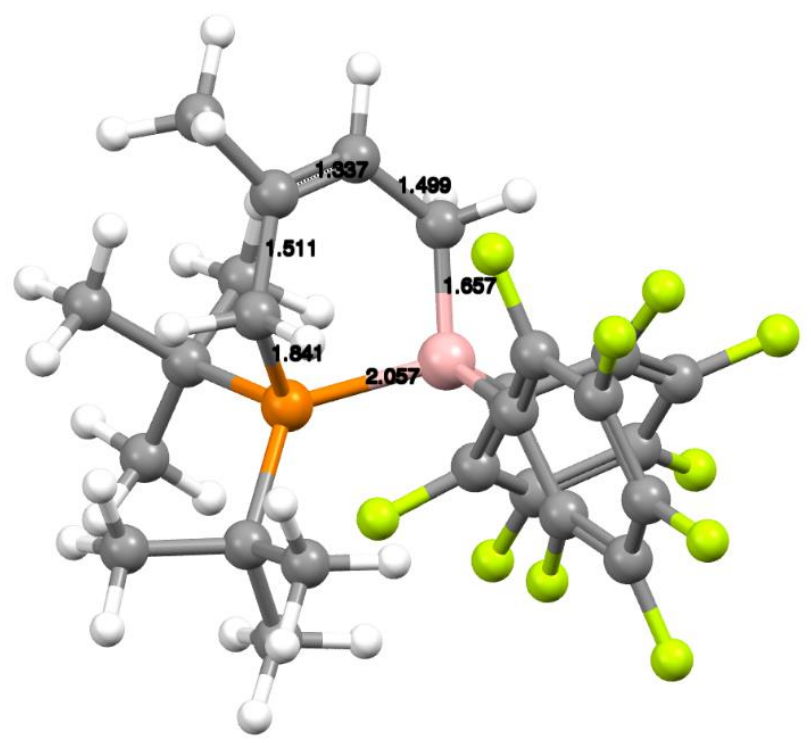

4b

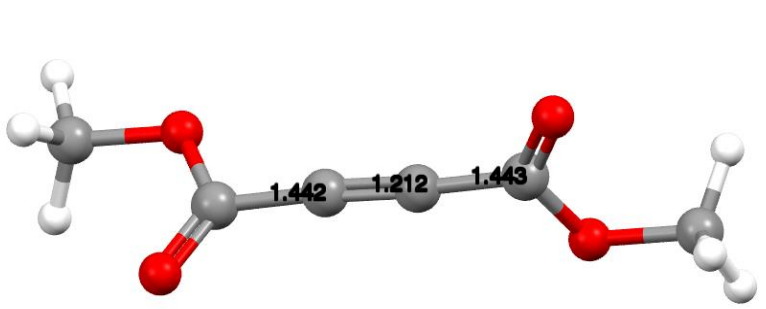

5b

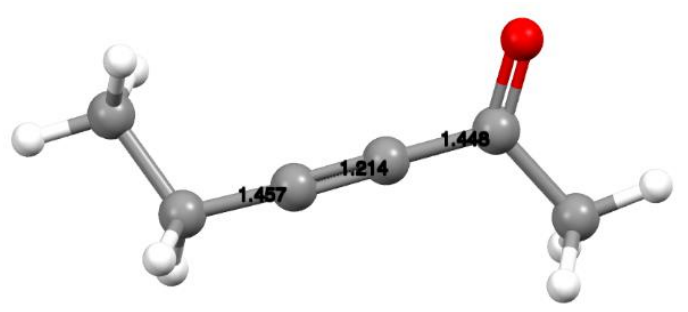

9b

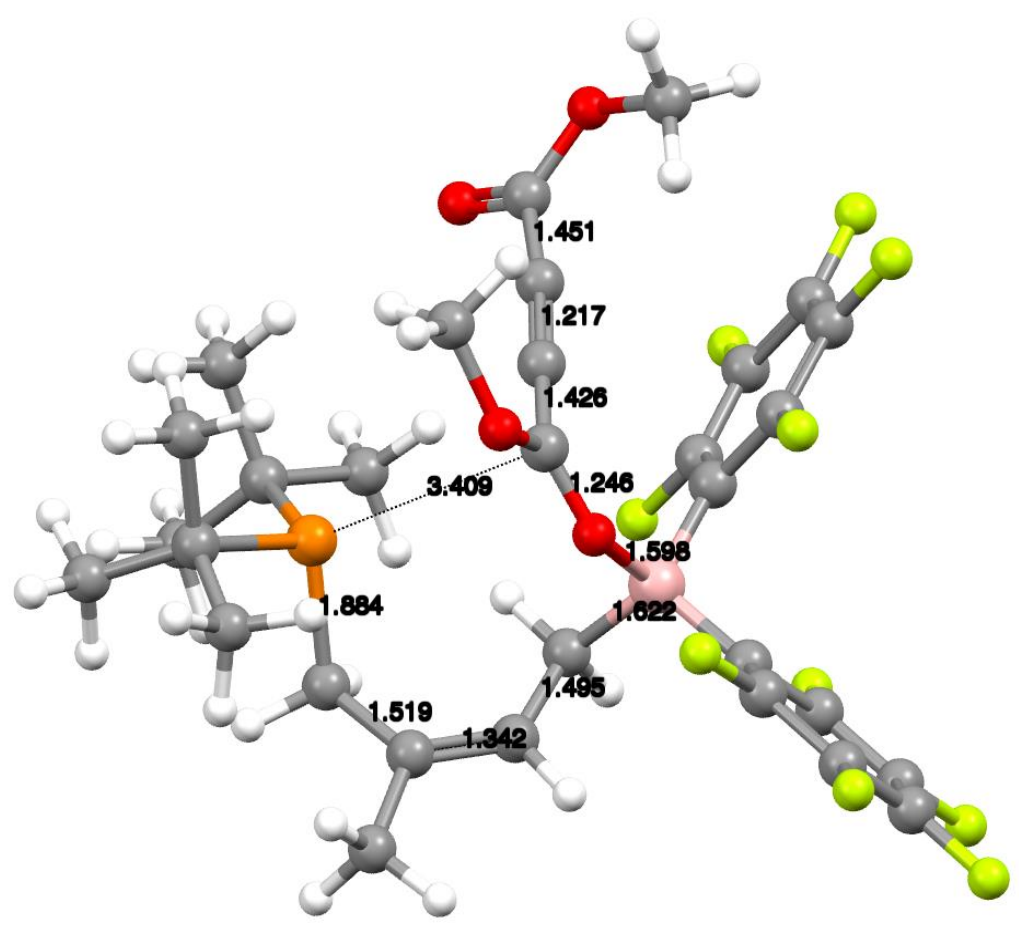

$(4 b \cdot 5 b)$ 
Figure S79 (continued)

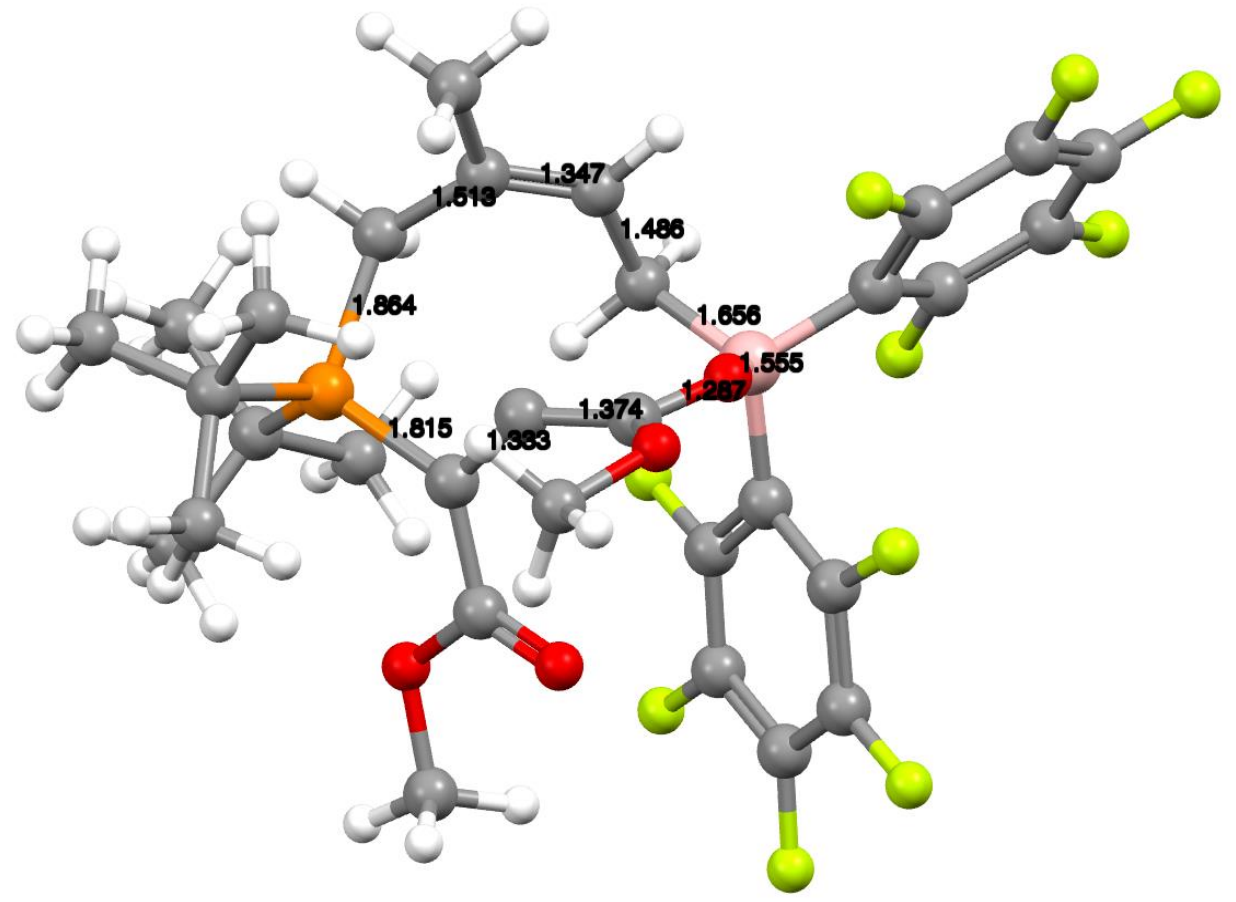

7d

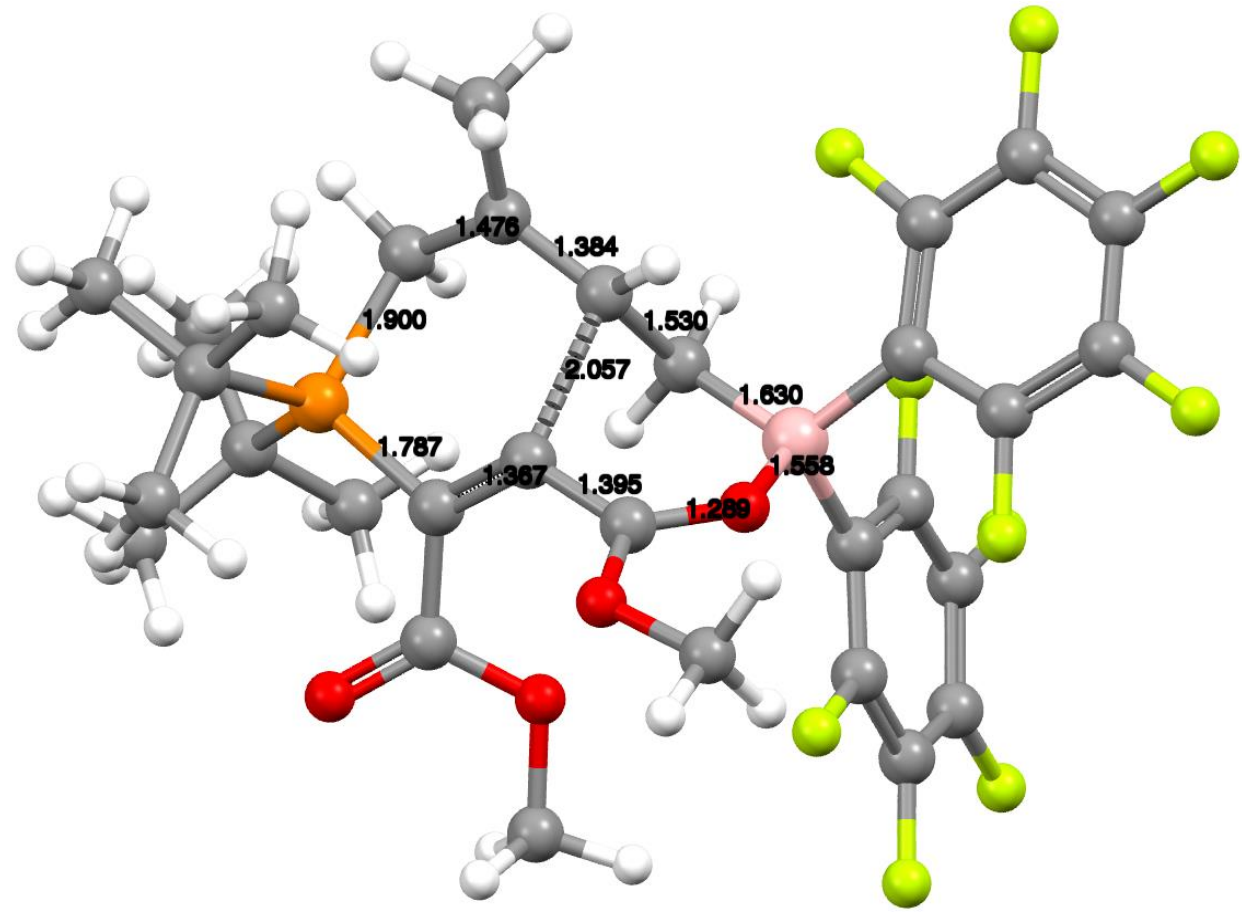

TS 7d-13a 
Figure S79 (continued)

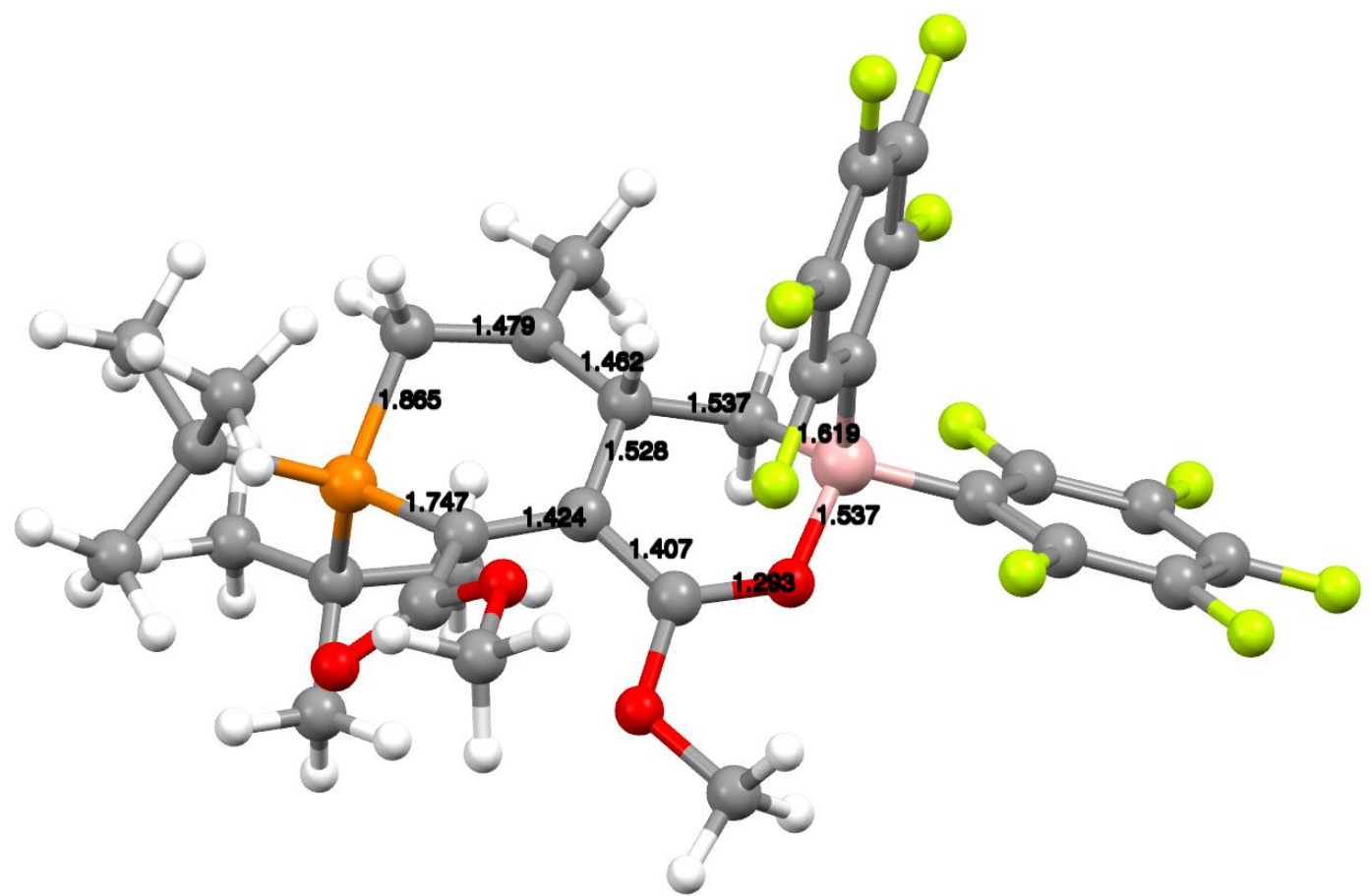

13a

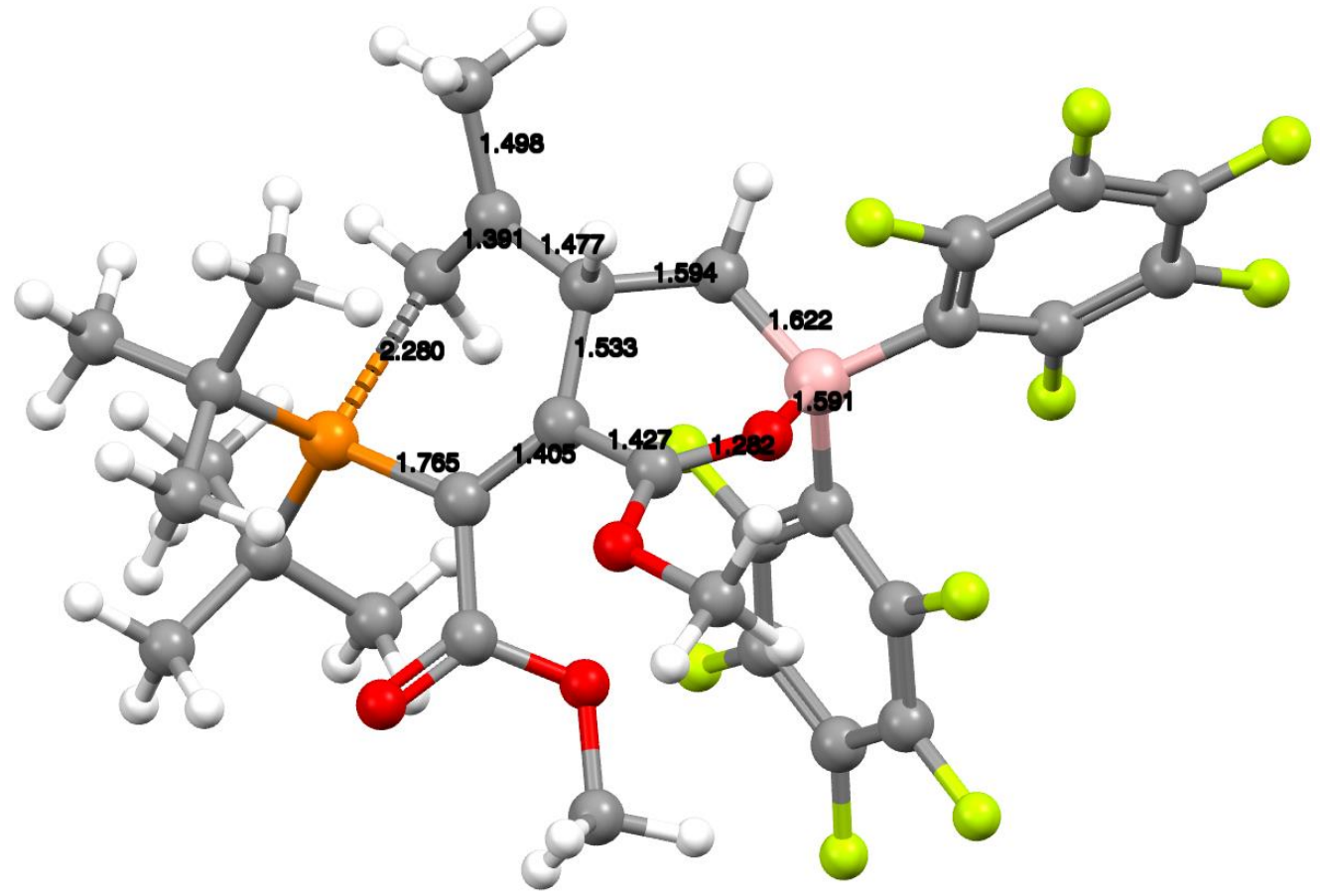

TS 13a-8d 
Figure S79 (continued)

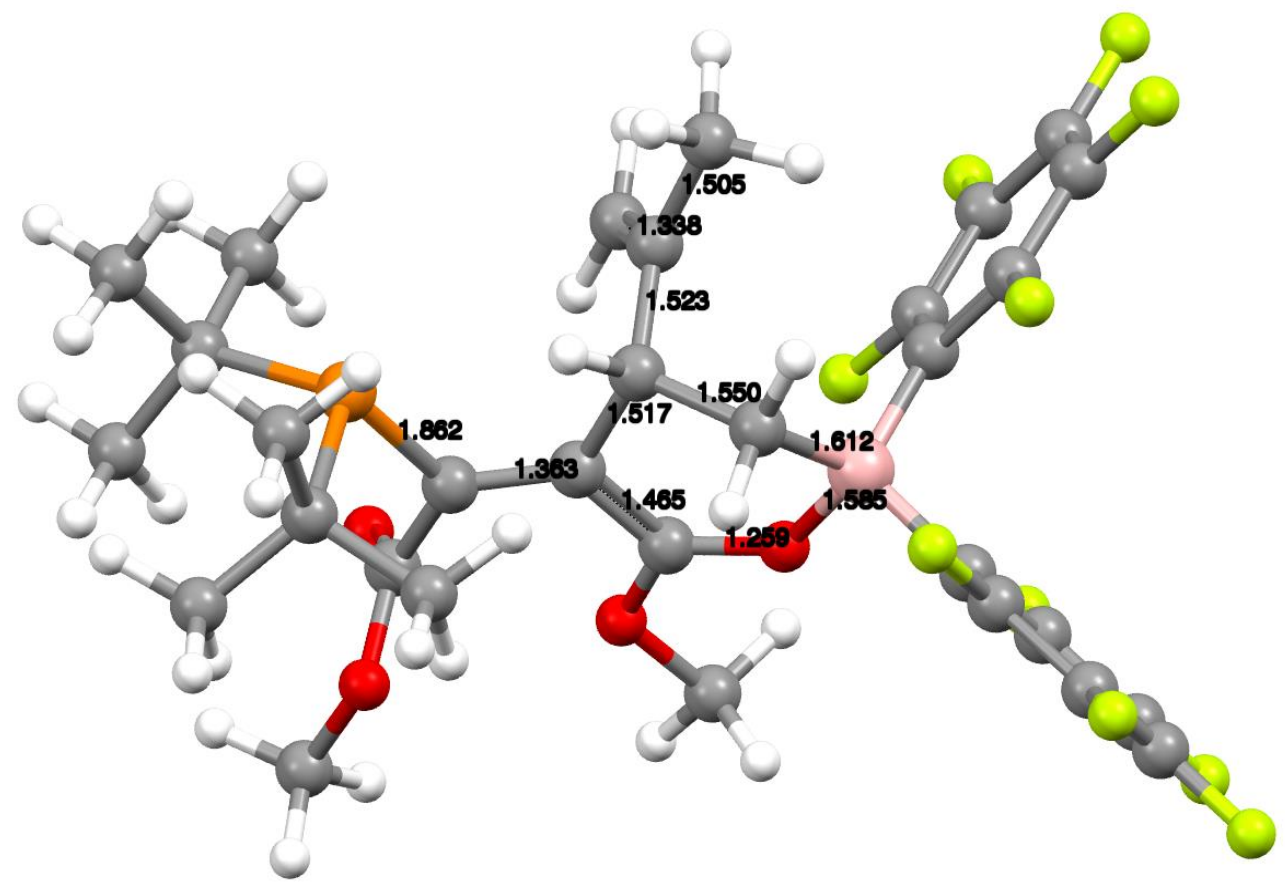

8d

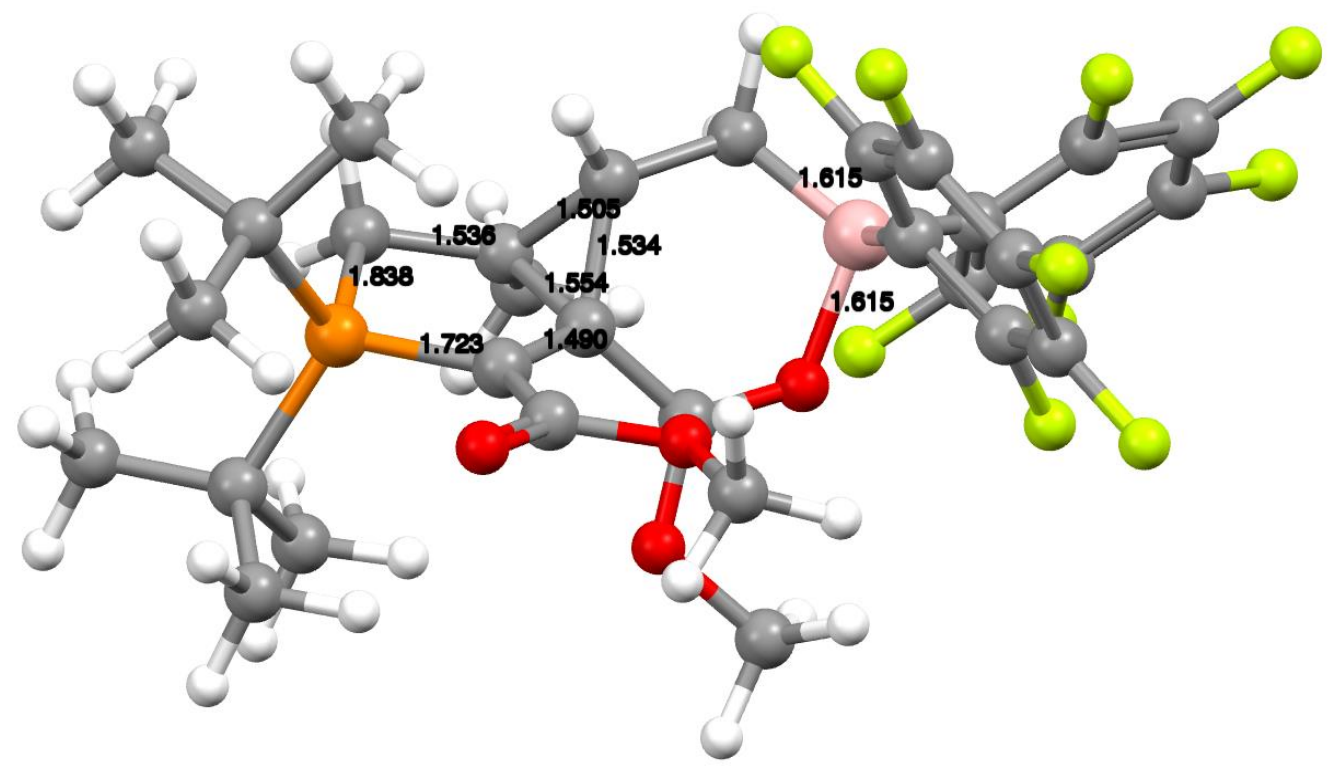

12 
Figure S79 (continued)

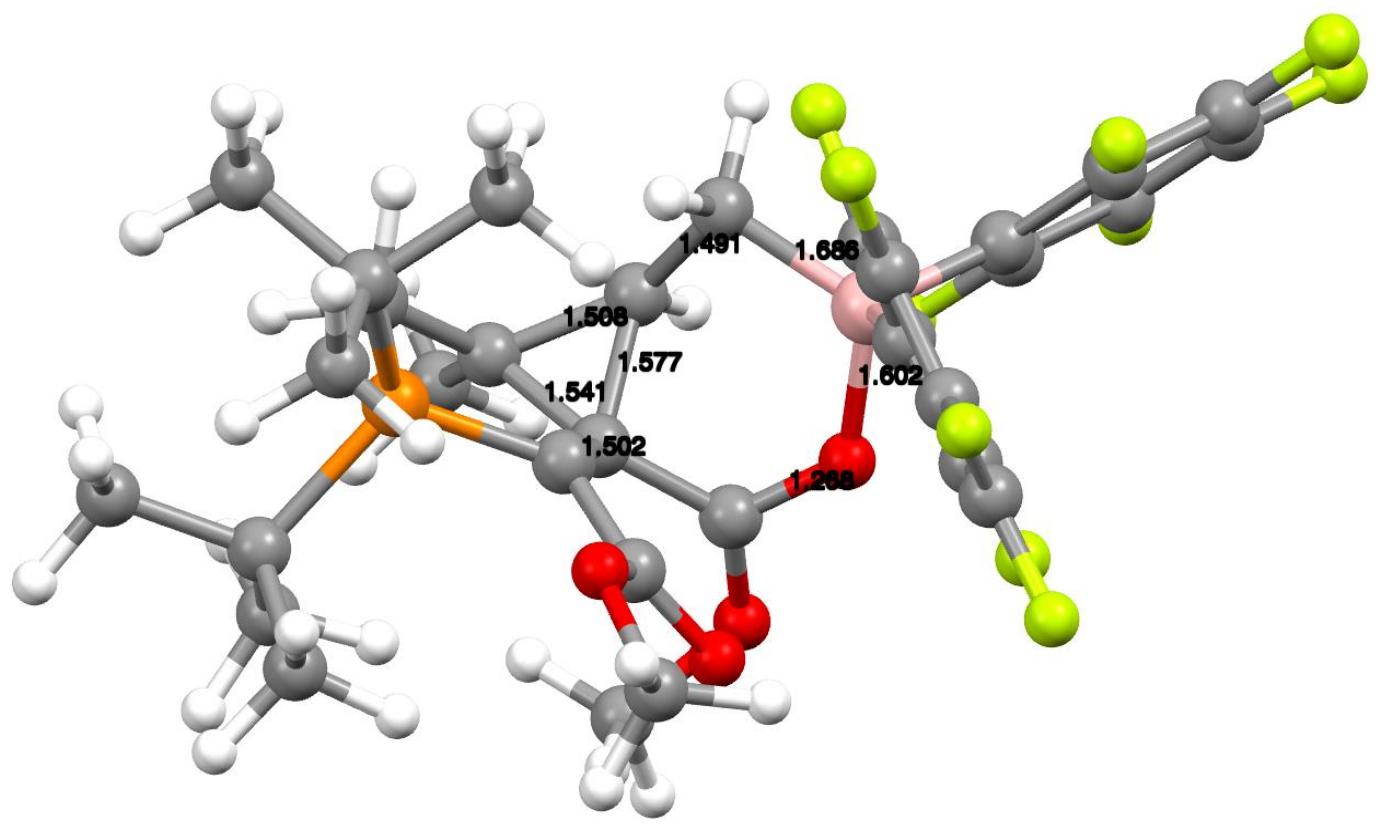

epi-12

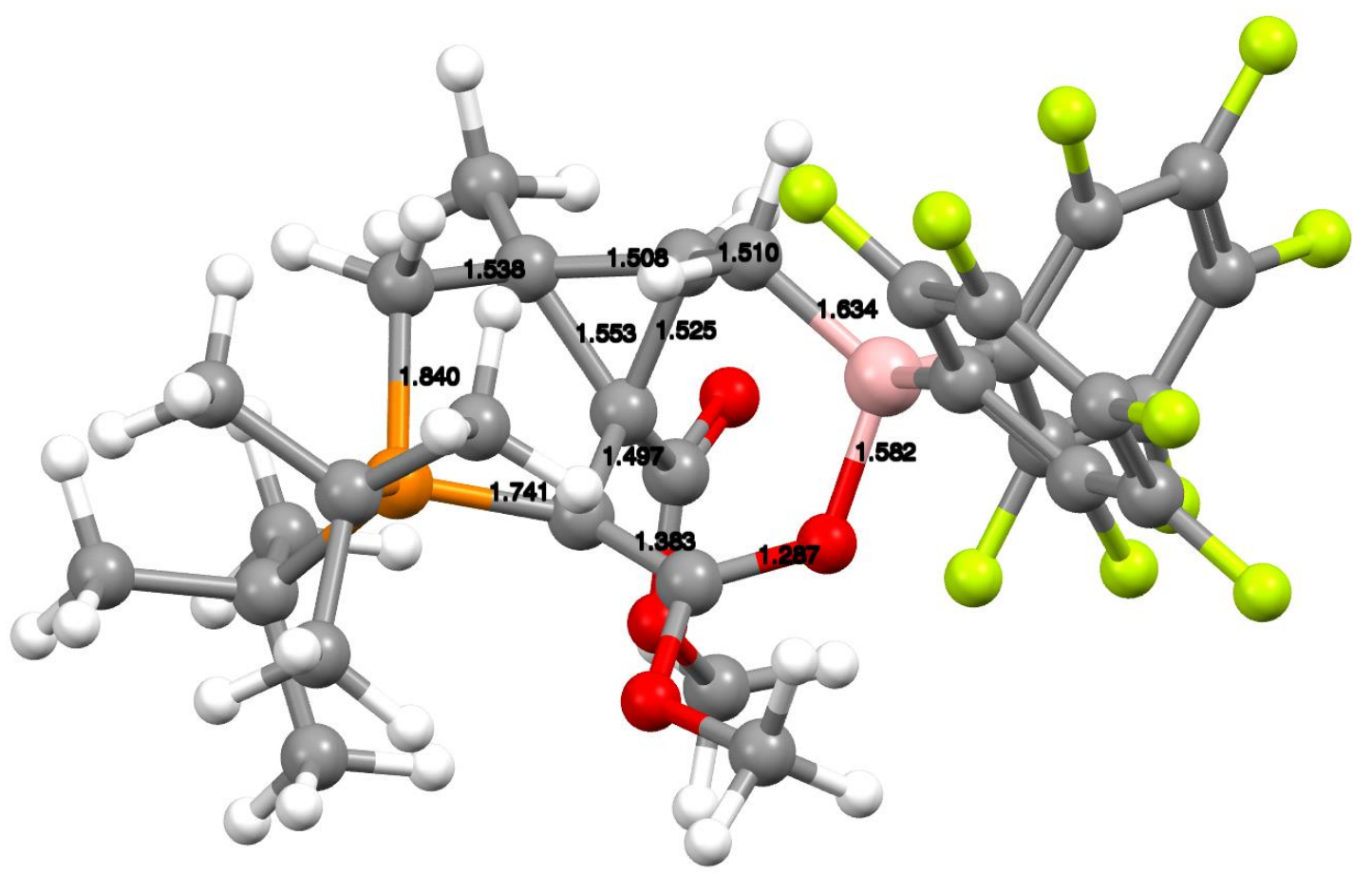


Figure S79 (continued)

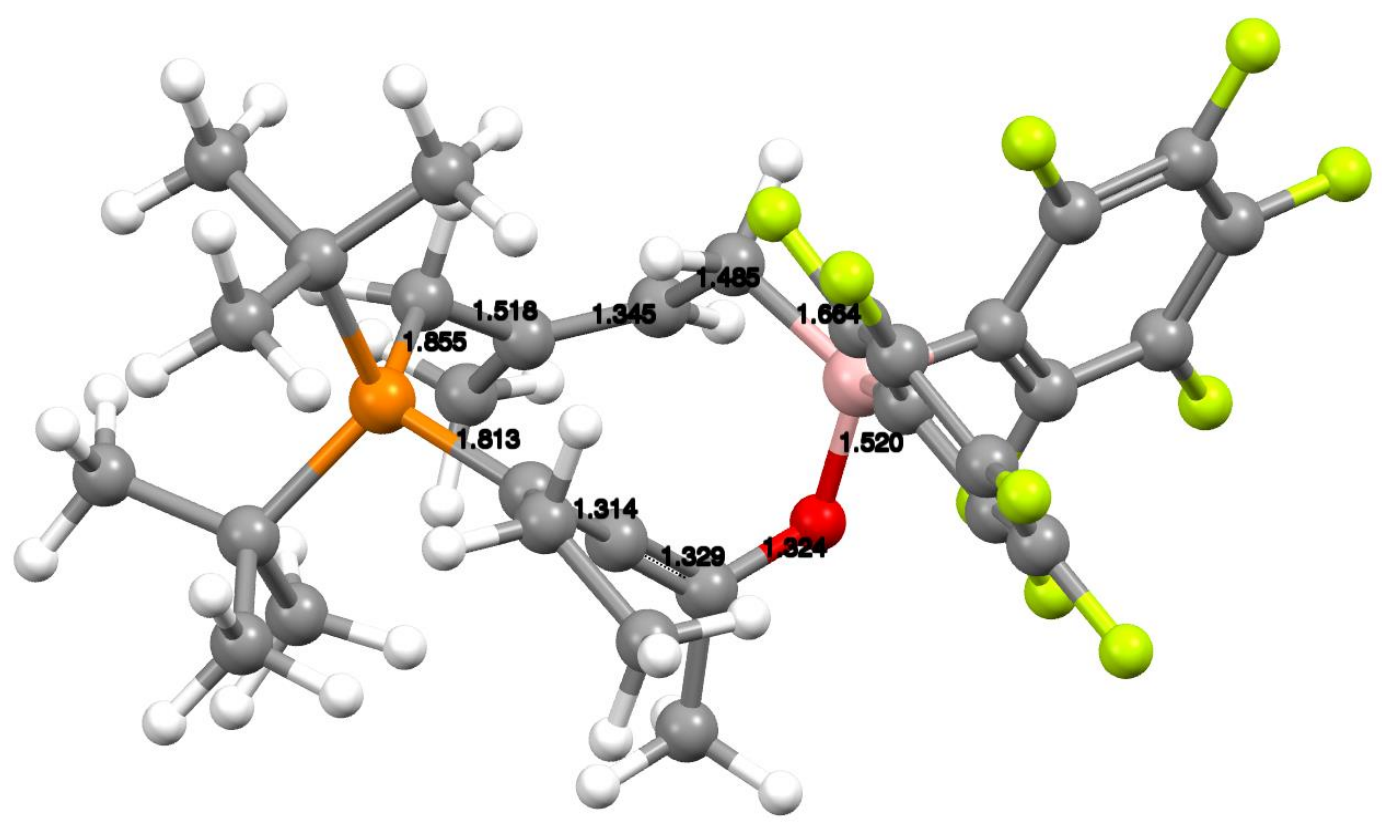

$11 a$

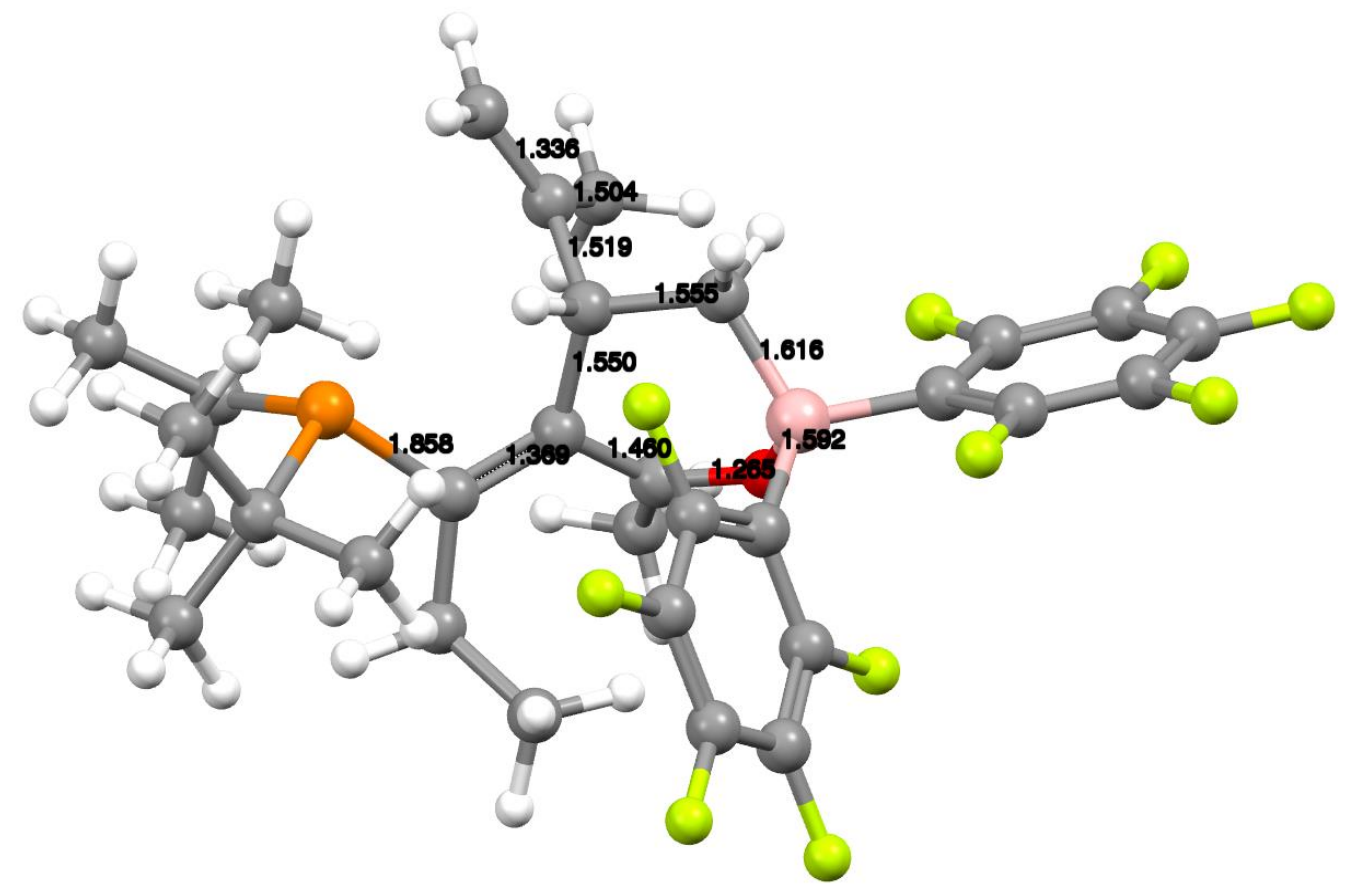

"8d-analogue" 
Figure S79 (continued)

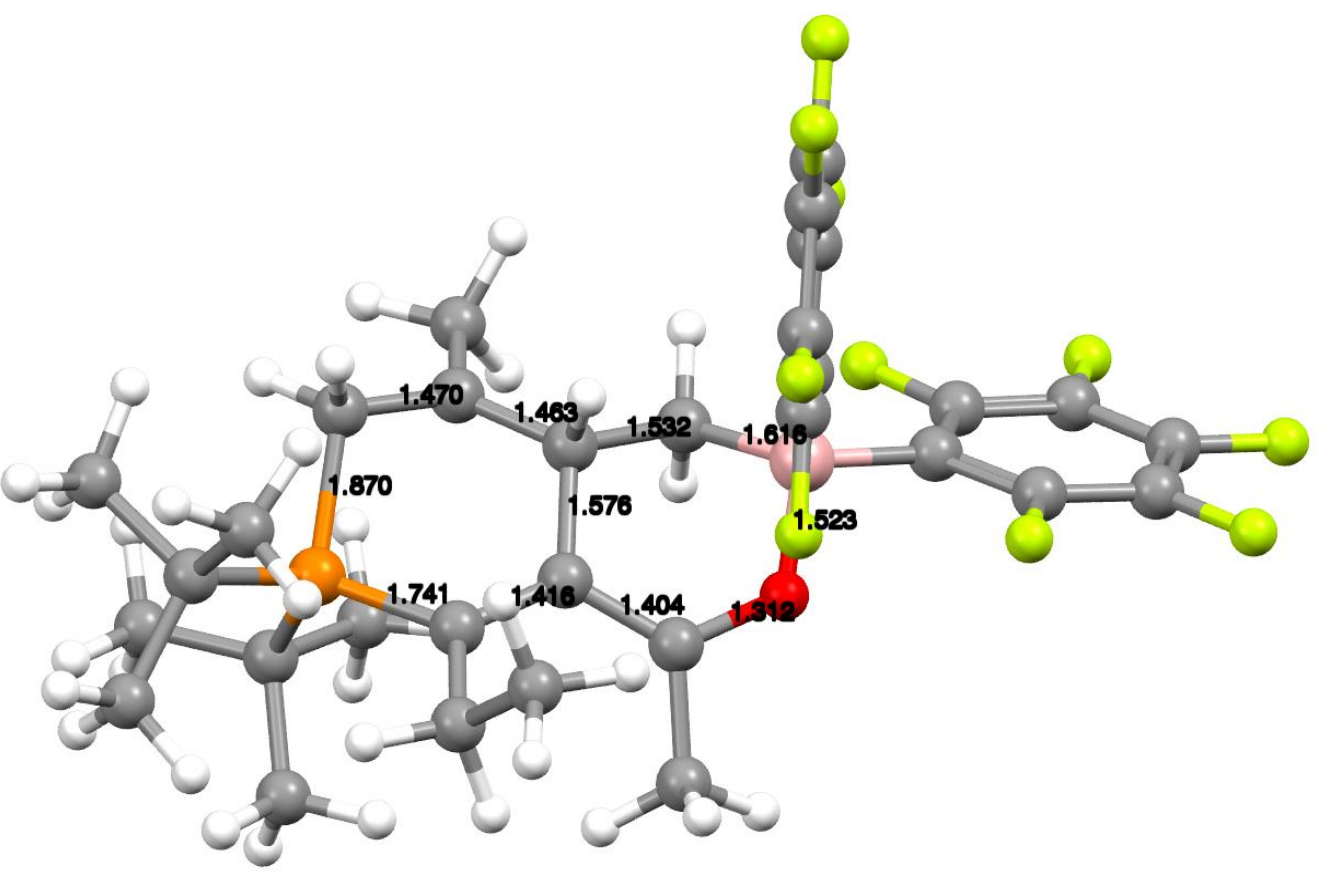

"13a-analogue"

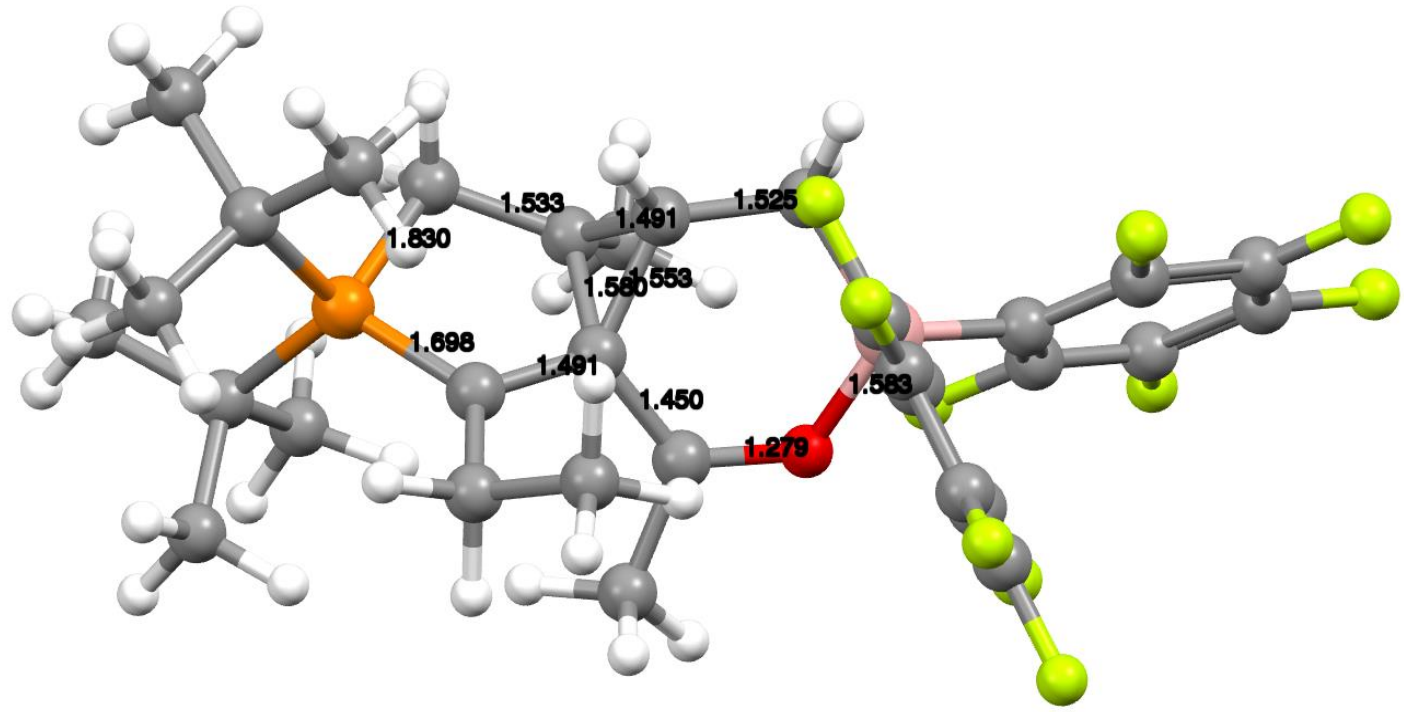

"12-analogue" 
Figure S80: Relaxed Potential Energy Surface (PES) scans (TPSS-D3/def2-SVP) for the C-C bond formation of the central cyclopropyl ring of 12 from 13a. In the two scans, the C-C bond was (a) varied from $1.55 \AA$ to $2.55 \AA$ (opening from 12) and (b) from $2.58 \AA$ to $2.18 \AA$ (closing from 13a). The energies of TS13a-8d and of 13a are depicted as a blue line and yellow square, respectively.

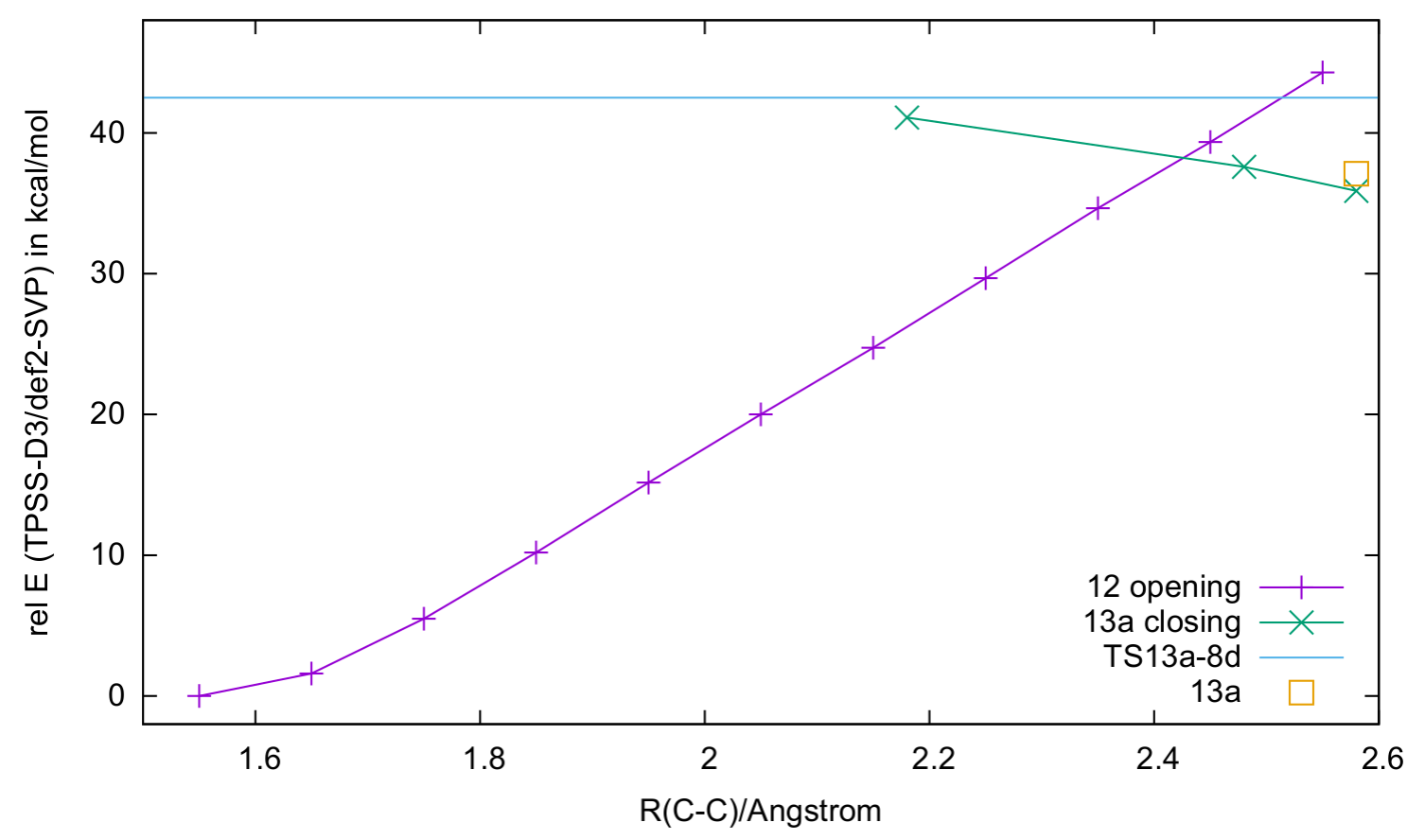

As can be seen from the curves in Figure S80, the reaction seems to involve the crossing of two electronic states which have a high probability of interconversion at a C-C distance of ca. $2.4 \AA$. The spin-restricted single reference method used for all other intermediates and transition structures of the mechanism is not capable of locating this transition state (TS13a-12). However, the crossing point of the two PES scans lies well below the transition structure TS13a-8d, which has a relative energy $\Delta \mathrm{E}$ of $+42.5 \mathrm{kcal} / \mathrm{mol}$ with this basis set.

1 TURBOMOLE V7.0 2015, a development of University of Karlsruhe and Forschungszentrum Karlsruhe GmbH, 1989-2007, TURBOMOLE GmbH, since 2007; available from http://www.turbomole.com

2 F. Neese, The ORCA program system, Wiley Interdisciplinary Reviews: Computational Molecular Science, 2012, 2 , 73-78.

3 J. Tao, J. P. Perdew, V. N. Staroverov and G. E. Scuseria, Phys. Rev. Lett., 2003, 91, 146401.

4 a) S. Grimme, J. Antony, S. Ehrlich, H. Krieg, J. Chem. Phys. 2010, 132, 154104. b) S. Grimme, S. Ehrlich, L. Goerigk, J. Comput. Chem. 2011, 32, 1456-1465.

5 F. Weigend; R. Ahlrichs. Phys. Chem. Chem. Phys. 2005, 7, 3297-3305.

6 S. Grimme, Chem. Eur. J. 2012, 18, 9955-9964.

7 Y. Zhao, D. G. Truhlar, J. Phys. Chem. A 2005, 109, 5656-5667.

8 a) A. Klamt, J. Phys. Chem. 1995, 99, 2224-2235. b) F. Eckert and A. Klamt, COSMOtherm, Version C3.0; COSMOlogic GmbH \& Co. KG, Leverkusen, Germany, 2013. 